\title{
Self-Organization and Mechanics of Minimal Actin Cortices attached to Artificial Bilayers
}

\author{
Dissertation \\ for the award of the degree \\ Doctor rerum naturalium \\ of the Georg-August-Universität Göttingen \\ within the doctoral program \\ Physics of Biological and Complex Systems \\ of the Georg-August University School of Science (GAUSS)
}

\author{
submitted by \\ Markus Schön \\ from Kassel
}

Göttingen 2018 


\section{Members of the thesis committee}

Prof. Dr. Claudia Steinem

Institute of Organic and Biomolecular Chemistry,

Georg-August-University Göttingen

Prof. Dr. Sarah Köster

Institute of X-Ray Physics,

Georg-August-University of Göttingen

Dr. Florian Rehfeldt

Third Institute of Physics,

Georg-August-University of Göttingen

\section{Members of the examination board}

Prof. Dr. Claudia Steinem

Institute of Organic and Biomolecular Chemistry,

Georg-August-University Göttingen

Prof. Dr. Sarah Köster

Institute of X-Ray Physics,

Georg-August-University of Göttingen

Dr. Florian Rehfeldt

Third Institute of Physics,

Georg-August-University of Göttingen

Prof. Dr. Silvio O. Rizzoli

Department of Neuro- and Sensory Physiology,

University Medical Center Göttingen

Prof. Dr. Michael Meinecke

Department of Cellular Biochemistry,

University Medical Center Göttingen

Dr. Sebastian Kruss

Institute of Physical Chemistry,

Georg-August-University of Göttingen

\section{Date of oral examination}

$27^{\text {th }}$ September 2018 


\section{Declaration}

I, Markus Schön, hereby certify that my doctoral thesis entitled "Self-organization and mechanics of minimal actin cortices attached to artificial bilayers" has been written independently and with no other sources and aids than quoted.

Göttingen, 2018

Markus Schön 

"What I cannot create,

I do not understand" Richard Feynman, 1988 



\section{Table of Contents}

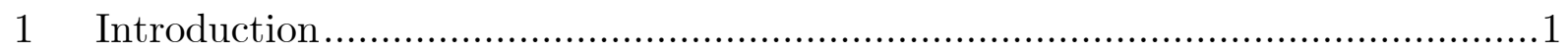

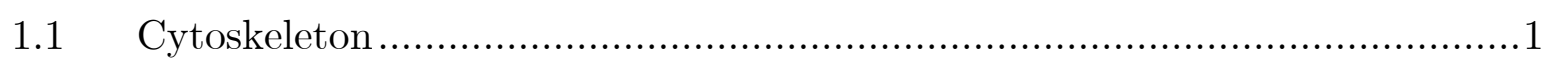

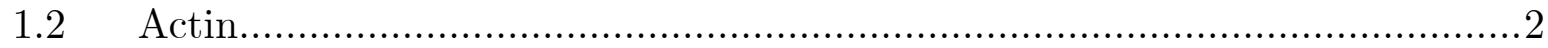

1.3 Actin Binding Proteins ............................................................

1.3.1 Ezrin - A Member of the ERM Protein Family ................................... 9

1.4 Minimal Actin Cortices - In vitro Model Systems ................................... 12

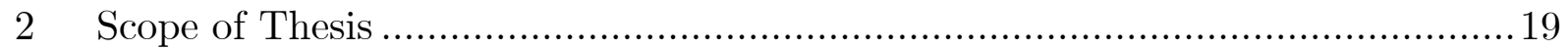

3 Materials and Methods ..................................................................... 21

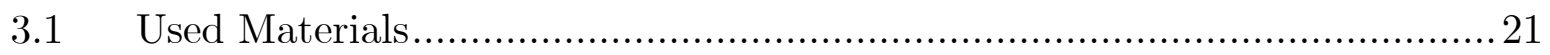

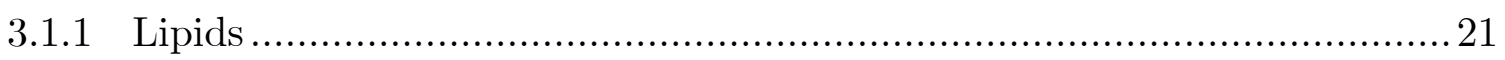

3.1.1.1 Matrix Lipids ....................................................................... 21

3.1.1.2 Receptor Lipids ................................................................ 21

3.1.1.3 Positively Charged Lipids ......................................................... 22

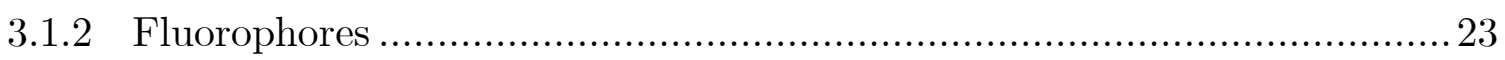

3.2 Biochemical Methods......................................................................... 26

3.2.1 Plasmid Transformation............................................................... 26

3.2.2 Plasmid Isolation ....................................................................... 27

3.2.3 Plasmid Sequencing................................................................ 27

3.2.4 Protein Expression ....................................................................... 28

3.2.5 Protein Purification ....................................................................... 28

3.2.6 Sodium Dodecyl Sulfate Polyacrylamide Gel Electrophoresis (SDS-PAGE) 30

3.2.7 Protein Labeling..................................................................... 31

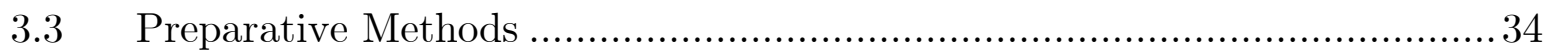

3.3.1 Preparation of Small Unilamellar Vesicles ..................................... 34

3.3.2 Preparation of Silicon Substrates used for Solid Supported Lipid Layers 35

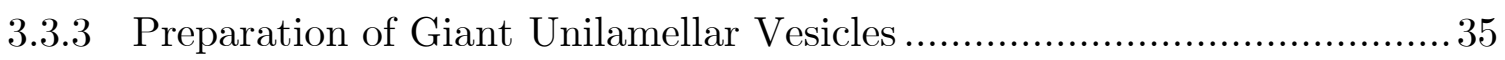

3.3.4 Preparation of Pore Spanning Lipid Bilayers.................................... 36

3.3.5 Polymerization of Actin-Networks ............................................... 38 
3.3.6 Preparation of Minimal Actin Cortices ........................................ 40

3.4 Bio-sensing Methods ............................................................. 41

3.4.1 Reflectometric Interference Spectroscopy (RIfS) ............................. 41

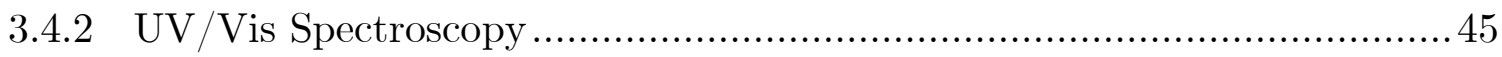

3.5 Biophysical Techniques.............................................................. 47

3.5.1 Atomic Force Microscopy ….............................................. 47

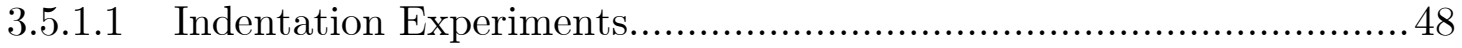

3.5.2 Confocal Laser Scanning Microscopy …........................................... 53

3.5.2.1 The Airyscan Technology …................................................. 56

3.5.2.2 Fluorescence Recovery after Photobleaching (FRAP) ....................58

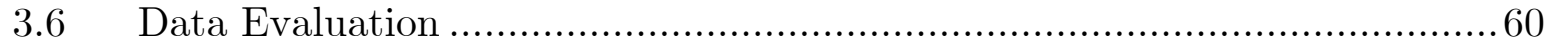

3.6.1 Skeletonization of Network Images ............................................ 60

3.6.1.1 Skeletonization via Artificial Retina ........................................ 60

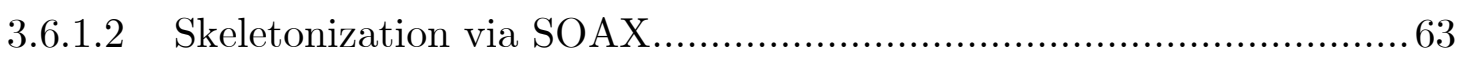

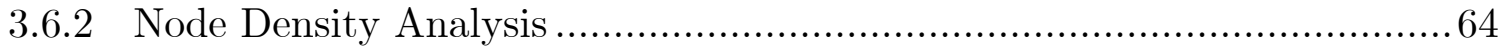

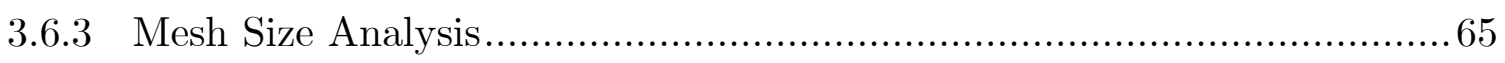

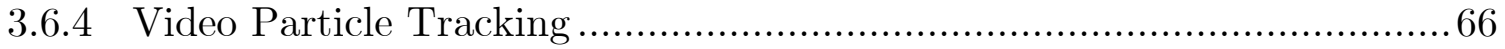

3.6.4.1 Data Processing .............................................................. 66

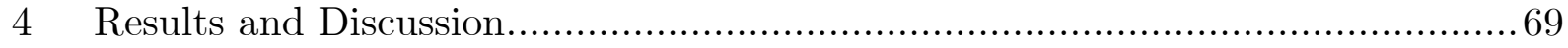

4.1 Characterization of Ezrin Binding as a Function of Pinning Point Density 69

4.1.1 Ezrin T567D Plasmid Isolation ................................................ 69

4.1.2 Isolation of Ezrin T567D ...................................................... 70

4.1.3 Characterization of Ezrin Binding to Solid Supported Lipid Bilayers as a Function of PtdIns $[4,5] \mathrm{P}_{2}$ Content.................................................. 71

4.1.4 Fluorescent Labeling of Ezrin T567D....................................... 79

4.2 Self-organization of F-actin Networks depending on the Pinning Point Density

4.2.1 Characterization of 3D pre-polymerized F-actin gels ........................... 81

4.2.1.1 Characterization of filament thicknesses by means of AFM............. 85

4.2.2 Attachment of F-actin Networks to Lipid Bilayers ............................... 87

4.2.3 Analysis of Self-Organization of attached MACs ............................... 92 
4.2.4 Effect of MAC attachment to the mobility of lipid bilayers................. 107

4.2.5 Discussion of Self-Organization of F-actin Attachment to Artificial Lipid

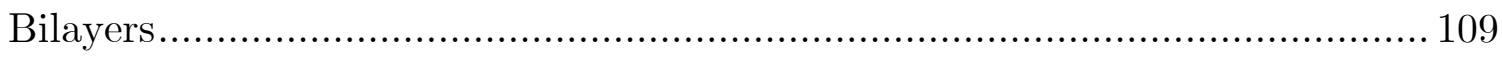

4.3 Mechanics of F-actin Networks........................................................ 113

4.3.1 Frequency-Dependent Viscoelastic Properties of F-actin Networks depending on Pinning Point Density .................................................... 113

4.3.1.1 Viscoelastic Properties of non-physiologically attached F-actin

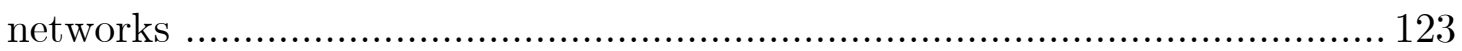

4.3.2 Force-Indentation Experiments on F-actin Networks......................... 127

4.3.2.1 Electrostatically Bound F-actin Networks ................................ 127

4.3.2.2 Conclusion of Force-Indentation Experiments with Attached MACs...

4.4 Alteration of F-actin Network Morphology by Actin Binding Proteins ..... 147

4.4.1 Influence of Fascin .............................................................. 147

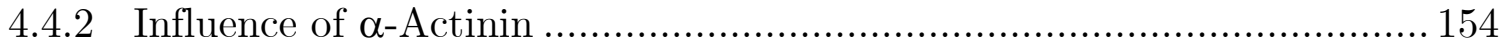

4.4.3 Discussion of the Influence of Actin Binding Proteins to MACs ............ 158

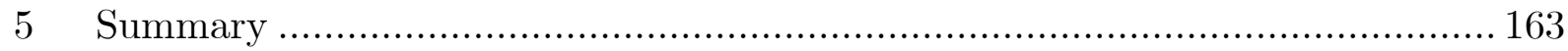

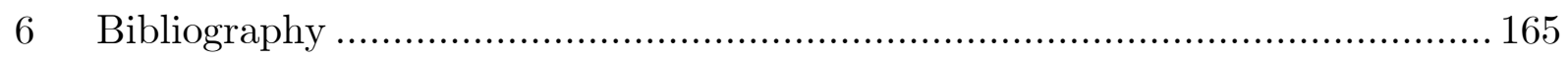

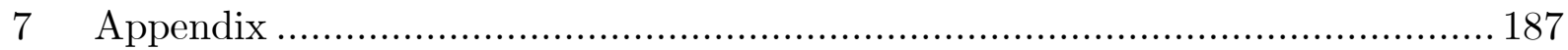

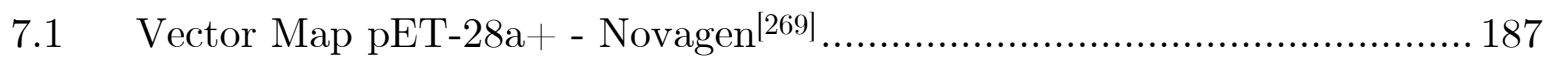

$7.2 \quad$ Used SOAX Parameters .................................................................... 188

7.3 Used Parameters to fit Rheological Data............................................ 188

7.3.1 Parameter Set considering One Cross-Link ..................................... 188

7.3.2 Parameter Set considering Two Cross-Links ..................................... 189

$7.4 \quad$ Used MATLAB Scripts ................................................................... 190

7.4.1 Bubble Mesh Analysis.......................................................... 190

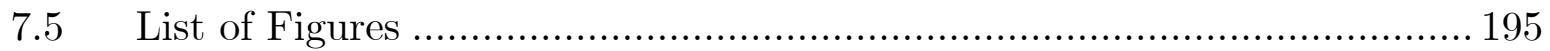

7.5.1 License Numbers of Retrieved Figures .......................................... 199

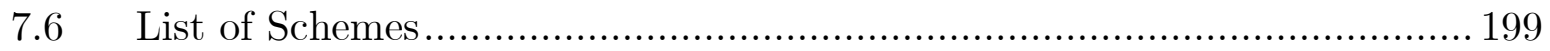

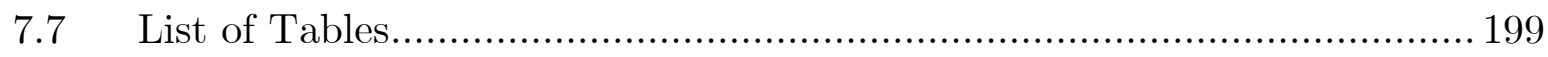

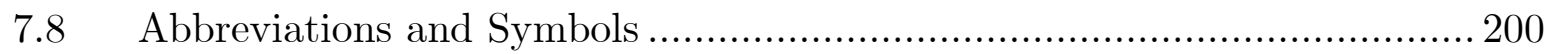

7.9 Chemicals and Consumables.......................................................... 203 
7.10 List of Devices and Software ..................................................... 206 
Abstract. One of the most versatile used protein structure in nature are actin filaments. They form contractile structures, like muscle fibrils, as well as complex network structures like the cellular cortex. The actin cortex is responsible for the shape, stability, mobility and further functions of the cell, which is realized by an interplay with over 100 accessory proteins. Concerning the shape and the mobility of cells, the connection between the actin cortex and the plasma membrane plays a pivotal role, which can be established by proteins of the ERM (ezrin-radixin-moesin) protein family. A vast number of proteins contribute to the variety of functions making it difficult to investigate the impact of the complex system's single components. To reduce the complexity, an artificial minimal actin cortex (MAC) was created, consisting of a lipid bilayer, the physiological linker protein ezrin and an F-actin network.

A dependency of the network density on the receptor lipid L- $\alpha$-phosphatidylinositol4,5-bisphosphate (PtdIns $\left.[4,5] \mathrm{P}_{2}\right)$ concentration in the membrane was found using confocal laser scanning microscopy (CLSM). Denser F-actin networks were observed, using a higher surface coverage of ezrin, induced by a higher concentration of PtdIns $[4,5] \mathrm{P}_{2}$ lipids. The network's height and the lateral fluidity of the lipid bilayer remained unaffected by an altered ezrin surface coverage. Interestingly, a filament sorting effect caused by network attachment was observed, which is apparent in decreasing filament lengths at higher densities of provided membrane connections. In general, smaller filaments were found in MACs in contrast to 3D F-actin gels.

To analyze the mechanical properties of MACs video particle tracking microrheology was performed. The overall stiffness of the network showed a 15 -fold increase in contrast to 3D F-actin network gels. An increasing density of anchoring points, induced by a higher PtdIns $[4,5] \mathrm{P}_{2}$ concentration, raised the stiffness further, proving that the rheological properties are governed by attachment of the network to the membrane. Indentation experiments on pore spanning membranes performed by means of atomic force microscopy showed that the lateral tension of the lipid bilayer can be altered by F-actin attachment. The influence on the membrane tension is strongly dependent on the resulting network morphology.

The developed and characterized MAC was then used as a tool to investigate the impact of the accessory proteins fascin and $\alpha$-actinin on the network morphology and the cortex dynamics by means of CLSM without interference from other proteins. Both proteins showed a rearrangement of the MAC: $\alpha$-actinin recruited actin from the flat MAC and established bundled, spider-web like structures on top of the flat F-actin cortex. Fascin bundled the actin networks showing increased filament lengths and a decreased network density. These results demonstrate that MACs physiologically attached via ezrin are dynamic enough to investigate the biological functions of single accessory proteins. 
Zusammenfassung. Eine der am vielseitigsten eingesetzten Proteinstrukturen in Zellen ist das Aktinfilament, welches beispielsweise lange Bündel, wie in Filopodia, oder Netzwerke, wie im Zellkortex, aufbauen kann. Der Zellkortex allein ist für viele Funktionen, wie die Stabilität, Form und Beweglichkeit der Zelle verantwortlich. Für die Erfüllung dieser Vielzahl an Funktionen wurden bereits über 100 Aktin assoziierte Proteine entdeckt. Dabei ist auch die Verknüpfung des Zytoskeletts zur Plasmamembran essenziell, welche über die Proteine der ERM (Ezrin-Radixin-Moesin) Familie erreicht werden kann.

Die Vielzahl der in vivo beteiligten Proteine erschwert die Untersuchung der Einflüsse einzelner Proteine. In der vorliegenden Arbeit wurde ein Modellsystem aus artifizieller Membran, dem physiologischen Protein Ezrin und einem filamentösen Aktin (F-Aktin) Netzwerk entwickelt, um den Einfluss einzelner Proteine studieren zu können.

Es konnte mit Hilfe von Fluoreszenzmikroskopie gezeigt werden, dass F-Aktin Netzwerke mit steigender Anzahl Membranverknüpfungen dichter werden. Die Anzahl der Verknüpfungen wurde durch Variation der Konzentration des Rezeptorlipides L- $\alpha$-Phosphatidylinositol-4,5-Bisphosphat (PtdIns[4,5] $\left.\mathrm{P}_{2}\right)$ und der hiervon abhängigen Oberflächenbelegung des Protein Ezrin verändert. Die Höhe des Aktinkortex und die laterale Mobilität der Membran bleiben dabei unverändert. Im Vergleich zu einem polymerisierten Netzwerk in 3D wurden im minimalen Kortex deutlich kleinere Filamentlängen gefunden. Bei steigender Anzahl der Verknüpfungspunkte werden diese zunehmend geringer, welches auf eine bevorzugte Anbindung kleinerer Filamente im minimalen Kortex-Modell hindeutet.

Mittels passiver Mikrorheologie konnte außerdem gezeigt werden, dass die membrangekoppelten Netzwerke eine 15-fach erhöhte Steifigkeit im Vergleich zu 3D Netzwerken aufweisen, welche mit steigender Verknüpfungszahl noch gesteigert werden kann. Zudem zeigten Indentationsexperimente mit Hilfe eines Rasterkraftmikroskops, dass die laterale Membranspannung durch das Anbringen eines Aktinnetzwerkes verändert werden kann. Diese Veränderung ist stark abhängig von der durch die Anbindung entstehenden Netzwerkmorphologie.

Der entwickelte und bereits untersuchte Aufbau des minimalen Aktinkortex wurde weiterverwendet, um den Einfluss einzelner aktin-bindendender Proteine auf den Kortex ohne den störenden Einfluss weiterer Proteine zu untersuchen. Während durch die Interaktion mit Fascin Bündel mit längeren Filamenten geformt wurden, zeigte die Zugabe von $\alpha$-Actinin, dass dieses Protein den Aktinkortex umstrukturiert und auf dem vorher flachen Kortex neue, Spinnennetz-artige Strukturen zusammenbindet. Diese Ergebnisse verdeutlichen, dass dieses biomimetische Modellsystem noch dynamisch genug ist, um biologische Funktionen einzelner Aktin-bindender Proteine schrittweise und individuell an diesen Minimalaufbauten zu untersuchen. 


\section{Introduction}

Research on the protein actin is moving more and more into the center of scientific attention, due to its various functions. It is mostly known for its activity in muscle cells in combination with myosin motor proteins, while actin is present in all eukaryotic cells, not only in the cytoplasm, but also in the nucleus.[1] Actin monomers (globular proteins, G-actin) are able to polymerize to filaments (F-actin) and can also depolymerize back to its globular form. This regulation, and furthermore the interaction between actin and actin binding proteins, like motor, bundling or capping proteins, creates dynamical processes which lead to corresponding functions. These functions are responsible for driving forces, transport processes, shape and stability of the cell. The latter one requires a close co-localization to the cell membrane, which is achieved in a merger with intermediate filaments and microtubule. This union is called the cytoskeleton.

\subsection{Cytoskeleton}

The three main proteins of the cytoskeleton, which are actin, intermediate filaments (IFs) and microtubules (MTs), are mostly decisive for the cell's spatial organization and its mechanical properties. ${ }^{[2]}$ All of them form filamentous structures and fulfill their own specific functions. Furthermore, evidence was found that all three types can team up to comply distinct functions. Different localizations and regions with spatial overlap of the three proteins are illustrated in Figure 1.1.
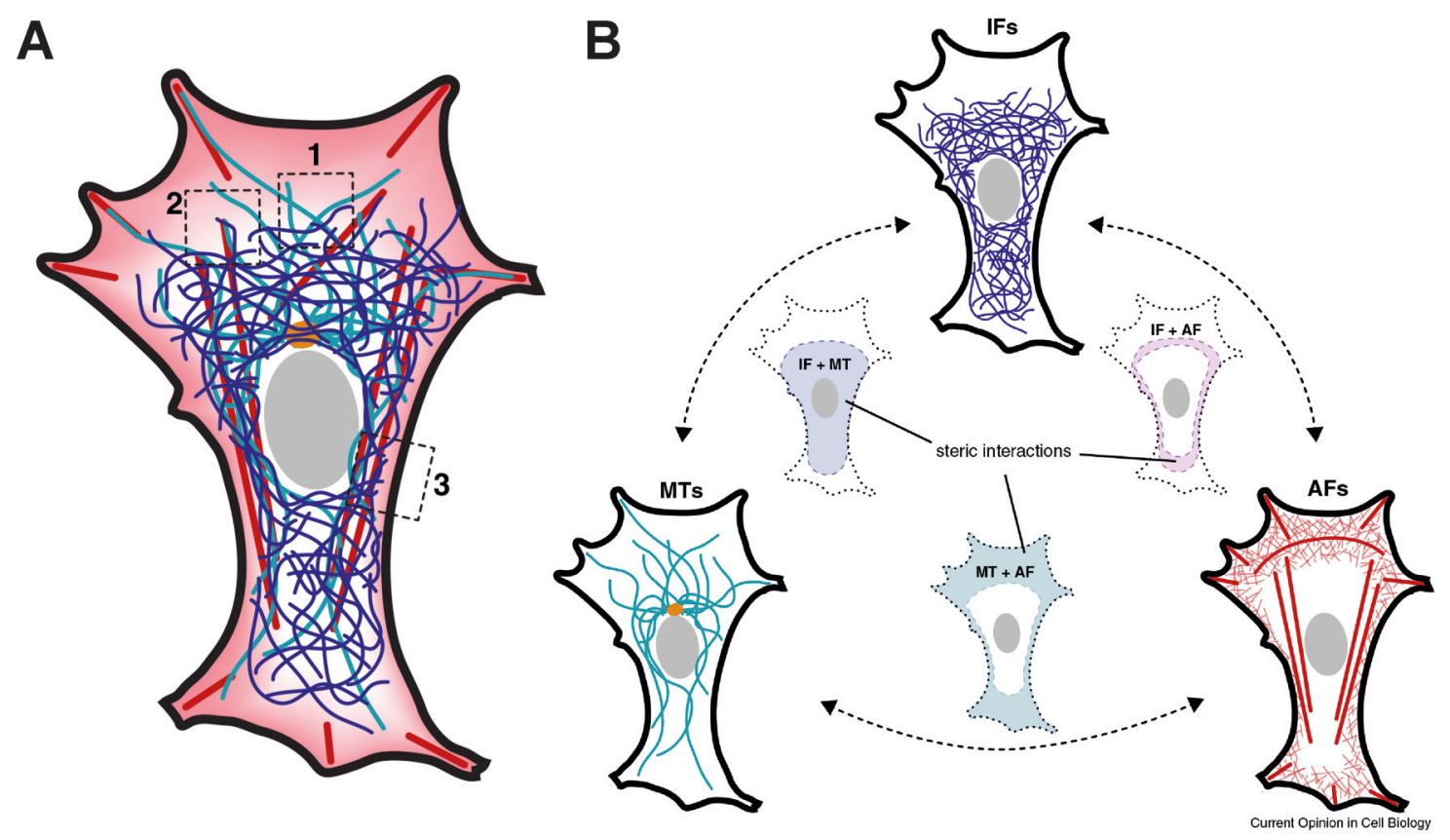

Figure 1.1: Localizations of cytoskeletal filaments and regions of their spatial overlaps. ${ }^{[3]}$ A: An overlap of cytoskeletal protein's organization within the animal cell is 
presented. Actin filaments (AFs) are shown in red, microtubule (MTs) in turquoise and intermediate filaments (IFs) in purple. B: When each type is illustrated separately the different spatial organizations of the three types become more obvious. In the center, the overlap of two filament types is shown. In each case there is a specific overlapping region where steric interaction occurs. Modified from Huber et al. and reprinted with permission from Elsevier. ${ }^{[3]}$

The interplay of all three types of proteins is highly effective, because all three filaments have different mechanical characteristics: ${ }^{4]}$ microtubules have the longest persistence length $\left(l_{p}\right)$ of about $1-5 \mathrm{~mm}$ and therefore provide the highest rigidity in the cytoskeleton. Intermediate filaments, consisting of Keratin and Vimentin, are the most flexible type of cytoskeletal filaments with $l_{p} \approx 0.3-2.0 \mu \mathrm{m}$. Most properties of actin filaments are generally in between the other types of filaments underlining its special role inside the cytoskeleton. These semi-flexible filaments with $l_{p} \approx 18 \mu \mathrm{m}$ are located in various parts of an epithelial cell, where they fulfill many different functions.

\subsection{Actin}

Actin filaments are not only found at special localizations of the cell, they also assemble in different types of organization: branched and highly dense filamentous networks (cf. Figure 1.2 I and III), anti-parallel contractile structures (cf. Figure 1.2 II) or parallel bundles (cf. Figure 1.2 IV).[5] These localizations paired with decisive organizations are necessary to accomplish several functions of the cell. For example, a flat, almost 2D layer of actin filaments, the cell cortex (Figure 1.2 I), covers the plasma membrane and is crucial for the maintenance of the cell shape. ${ }^{[6]}$ Thick, anti-parallel bundles, the contractile stress fibers (Figure 1.2 II), are connected with the plasma membrane via focal adhesion sites (purple) and are responsible for contraction of the cell. Contraction is generated by myosin motor proteins, which assemble between the anti-parallel filament bundles and "walk" along the actin filaments, whereby a sliding of the filaments occurs. ${ }^{[7]}$ At the cell's front, finger-like structures are present, the filopodia (Figure 1.2 IV), which are important for directional responses. ${ }^{[3]}$ Furthermore, a dense sheet of branched and linked networks is found at the cell's front, the so-called lamellipodium (Figure 1.2 III). Lamellipodia are the major engine of cell movement as these networks reorganize and push against the cellular plasma membrane. 


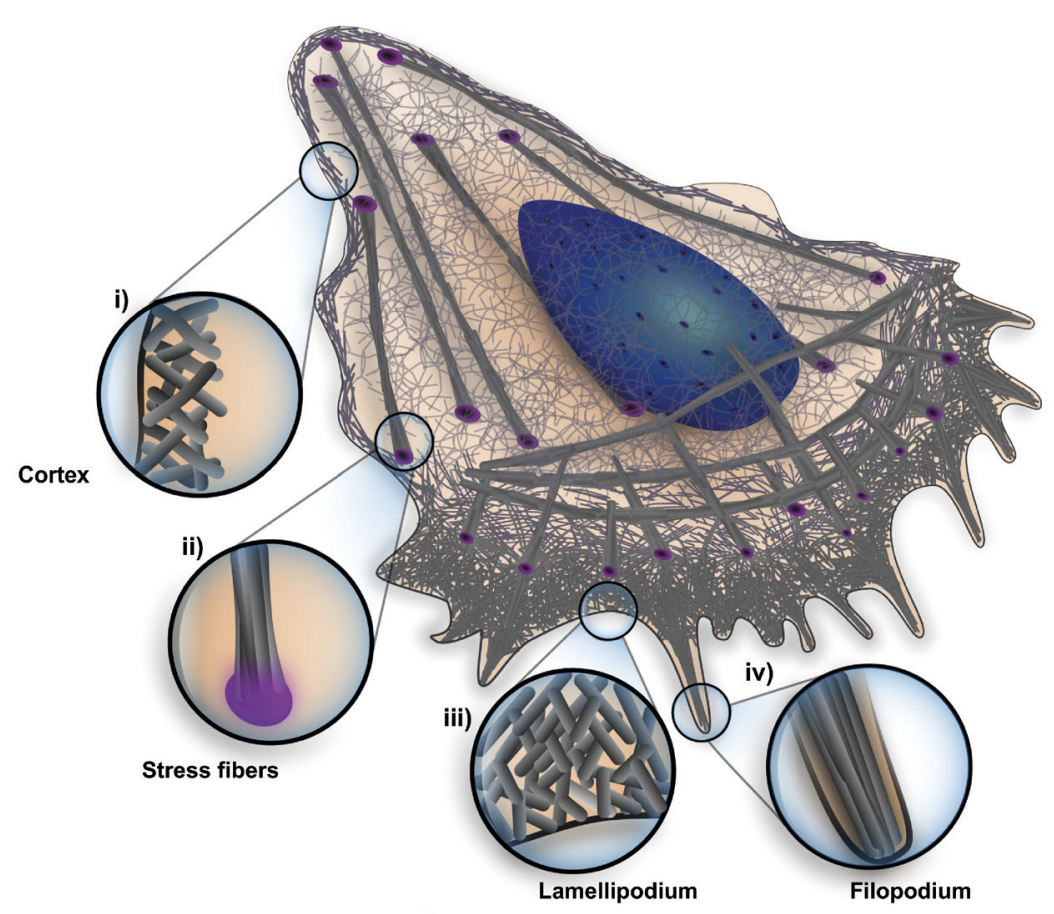

Figure 1.2: Actin localizations and organization in the animal cell. ${ }^{[5]}$ Actin filaments are found as bundled structures, as well as dense, branched networks. The plasma membrane is covered with a flat layer of a dense actin network, the actin cortex (I). At the front of the cell this cortex is more dense and dynamic, as it is the major engine of the cell's movement (III). Furthermore, in contractile stress fibers (II), anti-parallel bundles are found, while at the cell's front finger-like parallel bundles form the filopodia (IV). Modified from Blanchoin et al. ${ }^{[5]}$

Especially when the dynamic reorganization at the lamellipodia is considered, it becomes obvious that filamentous structures of actin cannot be only rigid rods. The machinery of actin's structural change relies on the assembly of single globular actin proteins (G-actin) which polymerize dynamically into filamentous polymers, so-called F-actin. [5]

The globular protein is not only the single most abundant protein in many eukaryotic cells, but also highly conserved among several species. For example, there is no change in any of the 375 amino acids present in the skeletal muscle isoform $\left(\alpha_{\text {skeletal }}\right)$ between humans and chicken. ${ }^{[8]}$ There are six isoforms of actin, four expressed primarily in skeletal $\left(\alpha_{\text {skeletal }}\right)$, cardiac $\left(\alpha_{\text {cardiac }}\right)$ and smooth $\left(\alpha_{\text {smooth }}\right.$ and $\left.\gamma_{\text {smooth }}\right)$ muscles, while two isoforms are expressed ubiquitously $\left(\beta_{\text {cyto }}\right.$ and $\left.\gamma_{\text {cyto }}\right)$. All isoforms share $93 \%$ identity resulting in proteins with a molecular mass of $M=43 \mathrm{kDa} .{ }^{[9,10]}$ Two specific binding sites within the globular protein are important for the polymerization process: first, a cation binding site and second, a nucleotide binding site where adenosine triphosphate (ATP) or adenosine diphosphate (ADP) can bind (cf. Figure 1.3).[11] Slight conformational disparities between ATP-actin, ADP-actin and nucleotide free actin were observed, which change the stability of the proteins: While ATP-actin is more stable than ADP-actin, the nucleotide free protein denatures rapidly and 
irreversibly. ${ }^{[12,13]}$ Therefore, cations and ATP induce the actin polymerization process of G-actin monomers, when monomers are present above a critical concentration.[14] The polymerization was found to be highly dependent on the $\mathrm{Mg}^{2+}$ concentration, therefore it is believed that $\mathrm{Mg}^{2+}$ binding at the cation binding site accelerates the polymerization. ${ }^{[15]}$
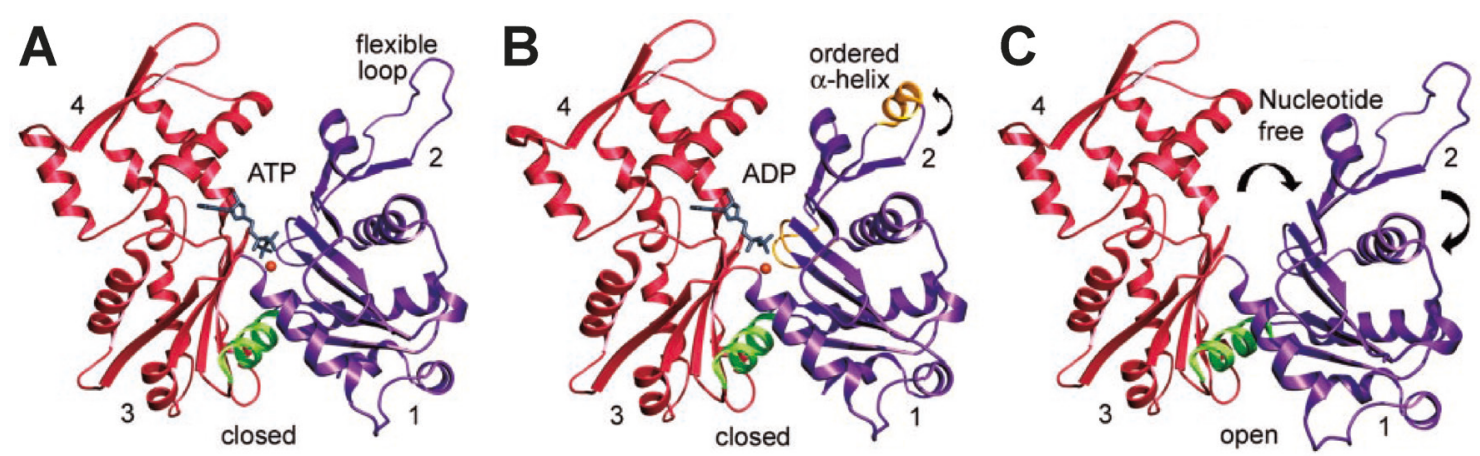

Figure 1.3: Three conformational states for a single G-actin monomer. ${ }^{[11]}$ A: Actin consists of four subdomains, 1 and 2 shown in purple, 3 and 4 in red. The domains 2 and 3 are connected by a linking $\alpha$-helix (green). Two subdomains on each side enclose the nucleotide ATP and a cation (orange). B: Inorganic phosphate $\left(\mathrm{P}_{\mathrm{i}}\right)$ release leads to conformational changes illustrated in yellow. A small rotation of subdomain 2 (indicated by an arrow) and a new ordered $\alpha$-helix are the most prominent changes. C: The model of the nucleotide free conformation shows two perpendicular rotations of about $12^{\circ}$ each (indicated by arrows). An opened cleft results between the subdomain 2 and 4. Modified from Graceffa et al. and reprinted with permission from the American Society for Biochemistry and Molecular Biology. ${ }^{[1]}$

The widely accepted actin polymerization model from Korn et al. proposes that three $\mathrm{Mg}^{2+}$-ATP-G-actin monomers assemble spontaneously to a nucleus.[16] Addition of more monomers first leads to a tetramer formation, which is subsequently followed by rapid elongation at both ends, to form a filament. At early stage, an ATP molecule within the oligomer can be cleaved, forming an interface between ATP-actin on one side and ADP.P $\mathrm{i}_{\mathrm{i}}$-actin subunit on the other side. After subsequent release of the phosphate, ADP-actin remains in the filament. At high monomer concentrations the elongation rate is faster than the ATP hydrolysis within the filament. As polymerization proceeds, monomer concentration decreases and the elongation rate becomes less, while ATP hydrolysis is independent from monomer concentration. ${ }^{[16]} \mathrm{A}$ steady state phase is reached whereby the filament continuously exchanges monomers at both ends. On one end, the barbed end (or (+)-end), the filament grows, because the monomer addition is faster than the removal, and on the other end, the pointed end (or (-)-end), the filament shrinks, due to faster removal than addition of monomers. As a result, the structurally polar filaments show a dynamic, but constantly in net length, treadmilling process. ${ }^{[17,18]}$ This process is highly dependent on the ATP concentration, since each change between ATP-actin, ADP.P $\mathrm{P}_{\mathrm{i}}$-actin and ADP-actin has different association or dissociation constants. ${ }^{[19]}$ However, it is assumed that the 
filament stability and turnover is controlled by ATP hydrolysis.[20] The finally polymerized actin filament (F-actin) consists of two chains, which form a right-handed helix. ${ }^{[21,22]}$ The actual symmetry of a single chain is left-handed as displayed in Figure 1.4. It demonstrates the structure derived from cryo-electron microscopy. ${ }^{[23]} \mathrm{A}$ single helix consists of 13 molecules and this structure repeats after six left-handed turns. The length of a single helix was determined to $36 \mathrm{~nm} .{ }^{[21]}$

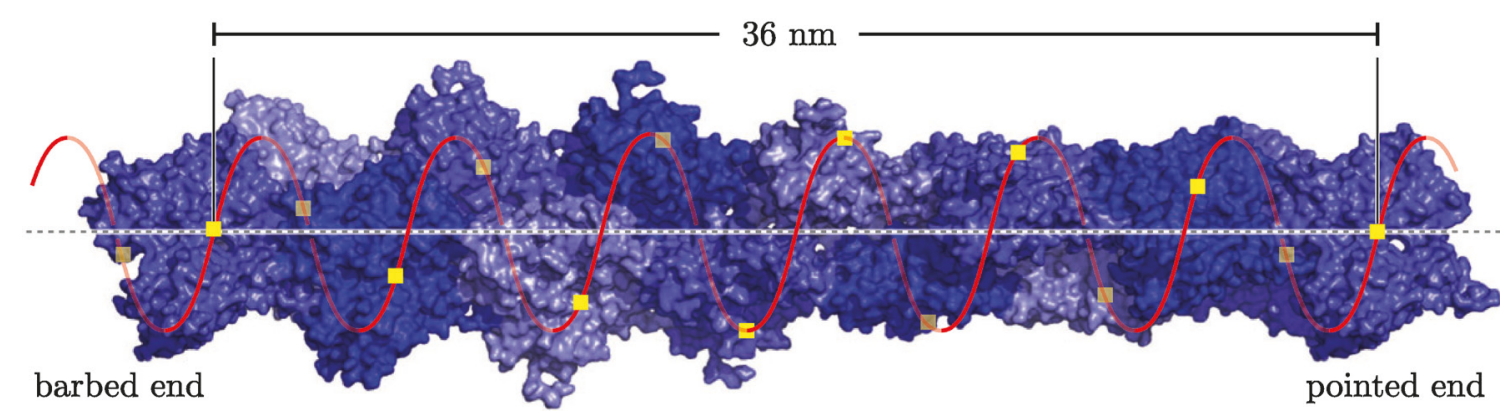

Figure 1.4: Helical structure of F-actin derived from cryo-electron microscopy. ${ }^{[21]}$ 13 molecules form a single, left-handed helix. The rise per molecule is about $2.76 \mathrm{~nm}$ which leads to a helix height of $36 \mathrm{~nm}$. After 13 molecules the structure repeats. Modified from Dominguez et al. and reprinted with permission from Annual Reviews, Inc. ${ }^{[21]}$

The resulting diameter of the right-handed double helical F-actin was found to be 5-7 nm. ${ }^{[24]}$ To maintain the pool of actin monomers and regulation of the assembly and turnover of actin filaments, additional accessory proteins are necessary. These accessory proteins were identified to cross-link, branch, bundle or cap the resulting actin filaments. Over time, it was found that eukaryotic cells have over 100 of these accessory proteins. ${ }^{[6]}$ Only in interaction with this vast number of actin binding proteins the filamentous actin network can accomplish the variety of functions and organizations within the cell.

Considering an artificial setup, an in vitro (lat.: in glass) approach, the treadmilling process of filamentous actin becomes a great challenge, as continuously polymerization had to be guaranteed. Therefore, a regulation of ATP and G-actin monomer concentration must be provided. To overcome this, it was shown that the use of the mushroom toxin phalloidin, a bicyclic heptapeptide, has been very useful in artificial experiments. ${ }^{25]}$ Phalloidin is interacting with the actin monomers within the filaments leading to a stabilizing effect against depolymerization. ${ }^{[26]}$ In addition to the stabilizing of actin filaments, it was shown that phalloidin accelerates the formation rate from G-actin to F-actin. ${ }^{27]}$ Subsequent studies showed, that there is no direct enhancing effect on the forward rate constant of polymerization, but the depolymerization rate constant is decreased in such a high magnitude that it experimentally could not be detected anymore.[28] A maximal stoichiometry of $1: 2$ with a $K_{\mathrm{d}}$ of $85 \mathrm{nM}$ was found.[29] Newer studies already revealed the position and orientation of phalloidin, located 
between two actin monomers within the filament. ${ }^{[30]}$ Fluorescent derivates of phalloidin were even more useful, as they stabilize F-actin, prevent depolymerization and visualize individual actin filaments in vitro. ${ }^{[31]}$ 


\subsection{Actin Binding Proteins}

The localizations and different organizations of F-actin is shown in Figure 1.2. Revisiting this scheme from the angle of a vast number of different participating actin binding proteins (ABPs), the complex network of actin filaments and its interplay with ABPs demonstrate how actin can be involved in many various functions (cf. Figure $1.5)$.

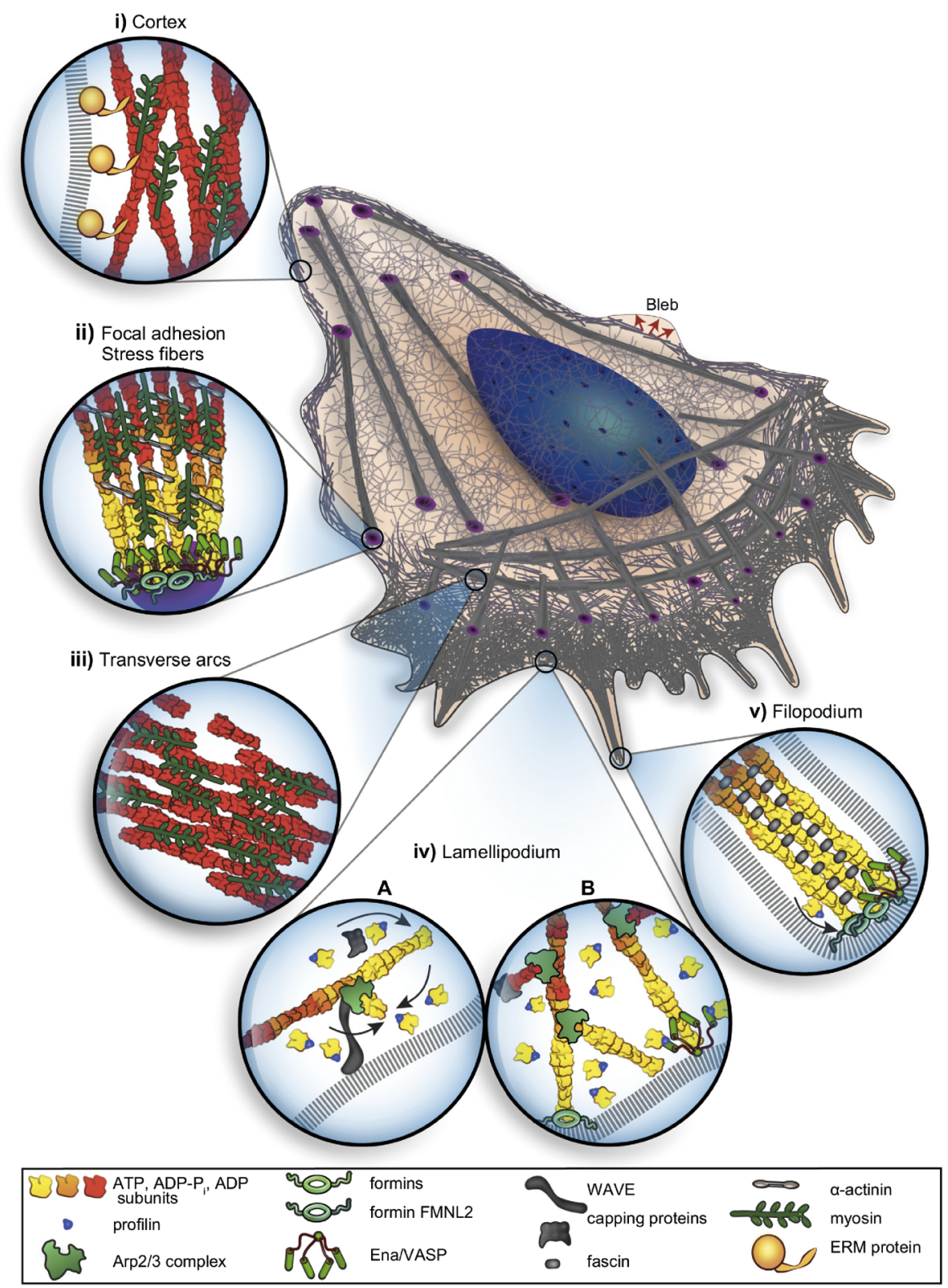

Figure 1.5: Specialized actin organizations and their interplay with several actin binding proteins..$^{[5]}$ I: At the cell cortex the actomyosin network (actin and myosin filaments) is connected to the plasma membrane via proteins of the ERM family. II: Ventral stress fibers, or anti-parallel bundles formed by cross-linking proteins such as $\alpha$-actinin, provide focal adhesion sites mediated by formins or VASP proteins. III: Transverse arcs are also contractile fibers which run parallel to the leading edge. IV: The highly dynamic lamellipodium shows branched networks. Branching is induced by the Arp2/3 complex, which is activated by the interaction with proteins of the WAVE family in turn. Elongation of the branched networks 
occurs by adding profilin/actin complexes to the barbed end of the filament (A). VASP proteins, the formin FMNL2 and capping proteins modulate the filament dynamics at the barbed end (B). V: Filopodia, the finger-like structures at the leading edge of the cell, are formed by tight, parallel actin bundles. Short distance bundles such as fascin can tightly pack these bundles. Modified from Blanchoin et al. ${ }^{[5]}$

Most filamentous structures and organizations of the F-actin network in vivo (lat.: with the living) are regulated or formed by these ABPs. For example, the finger-like filopodia at the leading edge of the cell or microvilli of epithelial cells are formed by parallel actin bundles (cf. Figure $1.5 \mathrm{~V}$ ). ${ }^{[32]}$ These tight bundles are formed by cross-linking proteins with a short distance between the two actin binding sites. Cross-linking in this context is defined as bridging of two actin filaments. ${ }^{[5]}$ One prominent example for short distance bundling is fascin (lat.: to bundle) which provides an actin-actin filament distance of 5-8 nm.[33] The resulting tight, parallel bundles face the protruded plasma membrane with their barbed ends. ${ }^{[34]}$ The cross-link distance range is therefore very important for the organization of the actin network as longer ranges, such as $160 \mathrm{~nm}$ for filamin, will not lead to bundling of actin filaments. ${ }^{[35]}$ The required cross-link distances for specialized functions explain partwise the variety of different ABPs found in nature. For instance, $\alpha$-actinin stabilizes actin bundles in an anti-parallel conformation with a distance range that myosin motor proteins can fit in between (cf. Figure $1.5 \mathrm{II}$ ), forming so-called stress fibers. ${ }^{[36]}$ Stress fibers are categorized into two main classes: transverse arcs, which run parallel to the leading edge of the cell (Figure $1.5 \mathrm{III}$ ), and ventral stress fibers, which run parallel to the direction of movement, linking focal adhesion sites (cf. Figure 1.5 II). Due to the fact that myosin is able to walk along the actin filaments, these bundles exert forces which lead to either contraction or disassembly. ${ }^{[5]} \alpha$-actinin also influences the function of stress fibers because it limits the extent of the contraction before disassembly is initiated. ${ }^{[37]}$

Furthermore, ABPs can interact and interfere with the polymerization process of actin to regulate the dynamics of the treadmilling process. For example, the protein profilin can bind G-actin monomers and inhibits the formation of dimers or trimers. As a consequence, a nucleation to form a new actin filament is avoided and the profilin bound G-actin monomers can only be used for assembly to existing actin filaments. ${ }^{[17]}$ The special binding site of profilin has a second key regulating effect: it can exclusively assemble at the fast-growing barbed end of the filament, while association at the pointed end is prevented (cf. Figure 1.5 IV). ${ }^{[38]}$ On the other hand, capping proteins, like gelsolin, can restrict the filament length by binding at the barbed end of the actin filament (Figure 1.5 IV).[39] Besides modulating the polymerization process or the filament length, ABPs can also modify the specific architecture of actin networks. The Arp2/3 complex consists of seven individual proteins and is able to induce branching of actin networks (cf. Figure 1.5 IV). ${ }^{[40]}$ The interaction of the actin filament with the 
Arp2/3 complex is only the beginning of branching, because a nucleating-promoting factor from the Wiskott-Aldrich syndrome protein (WASP/WAVE) family is necessary to activate the complex.[41] The growth of the new filament is promoted by assembling profilin-G-actin monomers (cf. Figure 1.5 IV A). The leading edge of these filaments was often found to protrude the plasma membrane and this was associated with Ena/VASP proteins and the activated formin FMNL 2.[42]

In addition to lamellipodia, the interaction with the plasma membrane is even more striking at the cell cortex (cf. Figure 1.5 I). The cortex consists of filamentous and bundled actin fibers in an interplay with active myosin motor proteins. This cortex is highly branched resulting in a mesh size of about 50-200 nm and extends around $200 \mathrm{~nm}$ vertically. ${ }^{[43,44]}$ This flat layer of an active actomyosin network is directly linked to the plasma membrane to establish a continuous force transmission between the plasma membrane and the actin cortex. Different proteins were found to be involved in this linkage, but the proteins ezrin, radixin and moesin of the ERM protein family are the most important players. ${ }^{[45]}$

\subsubsection{Ezrin - A Member of the ERM Protein Family}

The ERM (ezrin-radixin-moesin) family proteins are general cross-linkers between the plasma membrane and the actin cortex. ${ }^{[46]}$ The sequences of the $N$-terminal domains ( $N$-ERMAD, N-terminal ezrin-radixin-moesin associated domain) share around $85 \%$ of their identity, which is also similar to the human erythroid band 4.1 protein. ${ }^{46]}$ Therefore, this domain is mostly referred to as FERM (four-point-one and ERM) domain (cf. Figure 1.6 A, cyan). In most cultured cells the three proteins are co-localized and co-expressed but their distribution and expression appears to be regulated in a cell specific manner. ${ }^{[47]}$ This work will focus on ezrin, which was first isolated from microvilli of chicken breast in 1983.[48] Besides the already mentioned 298 amino acid FERM domain, ezrin's protein sequence shows an $\alpha$-helical domain (cf. Figure 1.6 A, yellow), a linker region and an around 80 amino acid large $C$-terminal ERM associated domain ( $C$-ERMAD, cf. Figure $1.6 \mathrm{~A}$, orange) which harbors an F-actin binding site identified in the last 34 residues. ${ }^{[49]}$ In particular, a threonine residue at position 567 within the $C$-ERMAD gets special attention as it was demonstrated, that phosphorylation of this threonine can lead to activation. ${ }^{[50]} \mathrm{A}$ two-step activation model of ezrin was proposed in which ezrin is recruited to plasma membrane regions enriched of L- $\alpha$-phosphatidylinositol-4,5-bisphosphate (PtdIns $[4,5] \mathrm{P}_{2}$ ), thus making the threonine residue more accessible (cf. Figure 1.6 B) for subsequent phosphorylation. ${ }^{[50-52]}$ This activation process is necessary because ezrin was found to exist as monomers and dimers in a dormant state. A binding site for the 
$C$-ERMAD was found in the FERM domain, which leads to either tight intramolecular binding or an intermolecular binding in an anti-parallel orientation. [53] The process is illustrated in Figure 1.6 B showing ezrin in a dormant state which is able to bind F-actin after the activation process of PtdIns[4,5] $\mathrm{P}_{2}$ binding and subsequent phosphorylation of T567.

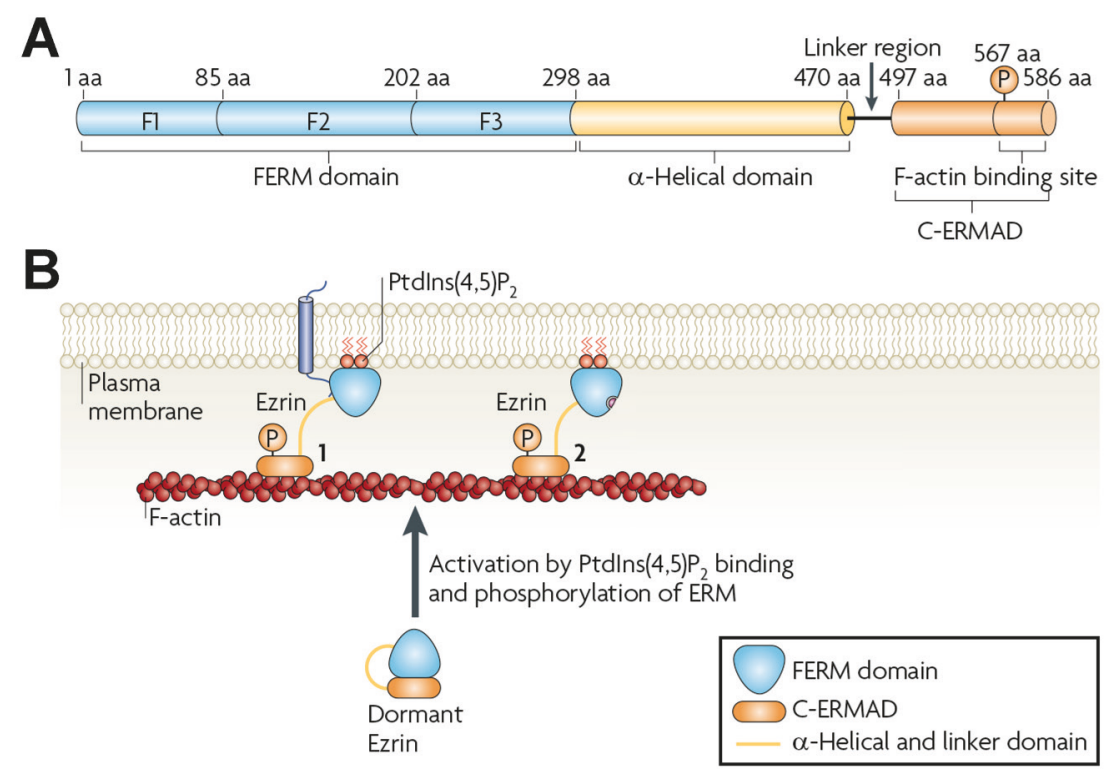

Figure 1.6: Ezrin domain organization and binding model based on a two-step activation process. ${ }^{[50]} \mathbf{A}$ : The $N$-terminal domain (FERM, four-point-one and ERM) of ezrin consist of three subdomains F1, F2 and F3 (blue). An $\alpha$-helical domain (yellow) and a 27amino acid linker region connect the $C$-terminal ERM domain ( $C$-ERMAD, orange) which harbors an F-actin binding site and a special threonine at position 567. B: This threonine has to be phosphorylated after initial ezrin activation from dormant state by $\operatorname{Ptd} \operatorname{Ins}[4,5] \mathrm{P}_{2}$ binding to complete the activation process. In the activated state ezrin cross-links the plasma membrane, via PtdIns $[4,5] \mathrm{P}_{2}$, with F-actin. Modified from Fehon et al. and reprinted with permission from Springer Nature. ${ }^{[50]}$

Both parts of the activation process are crucial for F-actin binding which was shown in in vitro experiments comparing the usage of an inactive ezrin mutant T567A and an active mutant T567D. ${ }^{[51]}$ The ezrin T567D mutant carries an aspartic acid instead of a threonine, introducing a negative charge at this position which results in a pseudo-phosphorylated mimetic protein. Many studies using this mutant showed that this pseudo-phosphorylated mutant is a useful tool for characterization of ezrin's functions in in vitro experiments. ${ }^{[45,51,54-57]}$

The for the activation process indispensable phospholipid PtdIns [4,5] $\mathrm{P}_{2}$ is most known for its participation in the phosphoinositide cascade, where $\operatorname{PtdIns}[4,5] \mathrm{P}_{2}$ is hydrolyzed by the phospholipase $\mathrm{C}$ into the second messengers diacyl glycerol and inositol-1,4,5triphosphate. These second messengers are subsequently responsible for a huge variety of signaling reactions. ${ }^{[58]}$ 
Previously, another important function of PtdIns[4,5] $\mathrm{P}_{2}$ was introduced: the activation and binding of ERM-proteins like ezrin, to establish a connection between plasma membrane and the actin cortex. Although PtdIns [4,5] $\mathrm{P}_{2}$ is a main regulator of functions in the plasma membrane, this lipid is a minor component, as it only comprises $1 \%$ of the phospholipids in human erythrocytes. ${ }^{[59,60]}$ It is assumed that $\operatorname{Ptd} \operatorname{Ins}[4,5] \mathrm{P}_{2}$ enriches locally but the underlying mechanism is still unclear. Proposed models extend from PtdIns[4,5] $\mathrm{P}_{2}$-platforms over 'selfish' PtdIns [4,5] $\mathrm{P}_{2}$ synthesis to spatially organized PtdIns[4,5] $\mathrm{P}_{2}$ 'mega pools'.[61] Some properties of PtdIns[4,5] $\mathrm{P}_{2}$ are unsuitable for these proposed models, for example the relatively high charge of $-3,-4$ or -5 . ${ }^{[59,62]}$

Returning to the actin cortex, a fluorescence study revealed that the $\operatorname{PtdIns}[4,5] \mathrm{P}_{2}$ density correlates with the density of the covering F-actin layer, while there was no co-localization for stress-fibers and PtdIns[4,5] $\mathrm{P}_{2}$ found. ${ }^{63]}$ In conclusion, although the mechanism is still unknown, an enrichment of PtdIns [4,5] $\mathrm{P}_{2}$ is known to initiate the activation of ERM proteins, which subsequently links the F-actin cortex with the plasma membrane. PtdIns $[4,5] \mathrm{P}_{2}$ is therefore the pinning point of membrane attachment of F-actin, which is mediated via ezrin (or ERM proteins in general). 


\subsection{Minimal Actin Cortices - In vitro Model Systems}

- Parts of this chapter have been published as Introduction of "Influence of cross-linkers on ezrin-bound minimal actin cortices" by M. Schön, I. Mey and

C. Steinem, submitted to Prog. Biophys. Mol. Biol. 2018, accepted ${ }^{[64]}$ -

In vitro model systems are a useful tool to investigate processes like actin network formation or cross-linking of the cellular cortex to a lipid membrane. Two main methodologies are mainly described: the top-down or the bottom-up approach. While the top-down approach starts with investigating complex systems and subsequent simplification of the model, the bottom-up approach starts with the simplest option. Considering the actin network, the investigation of a complex actin network with the vast array of different ABPs present, like in vivo, would describe a top-down approach. Otherwise, setting up a minimal system of an artificial lipid bilayer, consisting of only a matrix lipid and a functional one, and subsequent addition of a purely F-actin network, describes the bottom-up approach. These systems are referred to as minimal actin cortices (MACs) and have the advantage that each added component can be characterized by gradually characterization.

Typical setups for MACs are shown in Figure 1.7. Approaches are three-dimensional cell-sized vesicles which allow the MAC formation either on the inside (cf. Figure 1.7 A) or on the outside (cf. Figure $1.7 \mathrm{~B}$ ). A further strategy uses planar lipid bilayers to which MACs are bound resulting in an almost two-dimensional setup as shown in Figure 1.7 C.
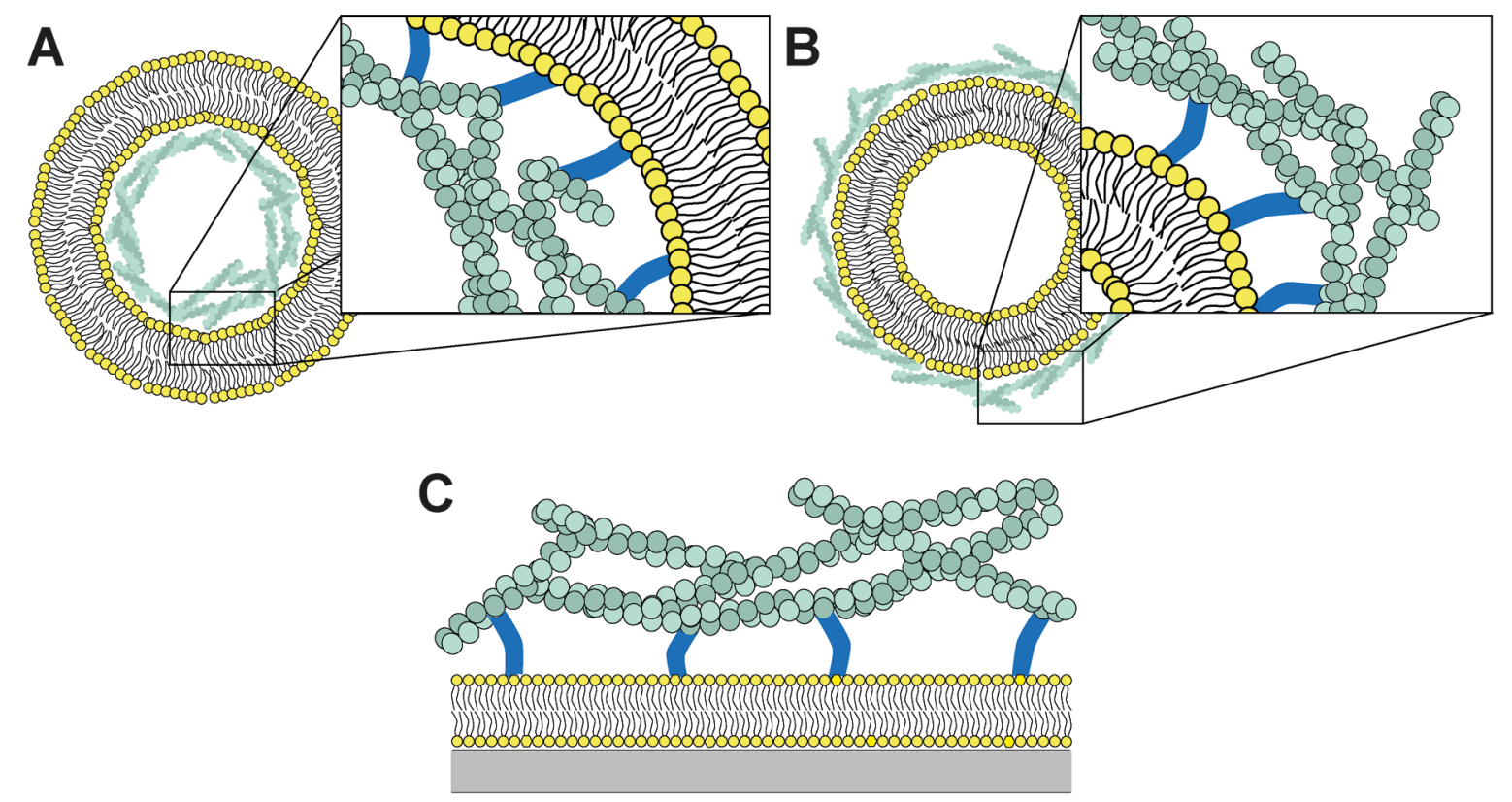

Figure 1.7: Schematic illustration of different arrangements of minimal actin cortices (MACs). ${ }^{[64]} \mathbf{A}$ : A vesicle or droplet with spatial expansion of a cell with an attached MAC (green) on the inside. For attachment a linker protein (blue) can be used. B: In a similar 
approach the MAC is attached on the outside of the vesicle mediated by a linker protein. C: If the lipid bilayer is spread on a support (grey) a planar geometry is achieved. The MAC can be attached using a linker protein. Retrieved from Schön et al. ${ }^{[64]}$

\section{MACs inside of vesicles or droplets}

One of the simplest approaches to mimic actin cortices is based on water-in-oil or oilin-water emulsions to form droplets, which allow for the formation of MACs. Shah et al. used a water-in-oil emulsion to self-organize a dynamic actin cortex at the inner interface. ${ }^{[65]}$ This system was reported to undergo spontaneous symmetry breaking guided by myosin-induced cortical actin movement outlining the dynamics of actin cortices in vivo. Such a system can provide information about cortex formation and symmetry breaking during cellular processes like division or initial polarization of the embryo. However, the interaction with oil lacks the physical characteristics of a lipid membrane, although the geometry of the cellular cortex is reproduced. Furthermore, the dynamics of protein-induced membrane coupling are neglected, which are important for e.g. the length scales of characteristic interactions in processes like actin-cortex reshaping by myosin. [66]

Already in 1989, a biomimetic MAC was created, using giant unilamellar vesicles (GUVs) as lipid bilayer and the F-actin cortex on the inside was created by polymerization and self-assembly of G-actin induced by valinomycin mediated $\mathrm{K}^{+}$ influx (cf. Figure 1.7 A).[67] It was observed that actin polymerization changes the vesicles' shape dependent on the actin filament length capped by addition of gelsolin. In a similar approach, the polymerization within GUVs was induced by $\mathrm{Mg}^{2+}$ influx via the ionophore A23187.[68] This setup revealed a proximity of F-actin layer and lipid bilayer without the usage of any membrane-F-actin coupling agent. In later studies it could been shown that bivalent cations like $\mathrm{Mg}^{2+}$ mediate weak coupling between F-actin and neutral phospholipids.[69] As a further development of this setup, cross-linking agents like $\alpha$-actinin and filamin were introduced. Both G-actin and the desired cross-link were encapsulated inside the GUV and polymerized by $\mathrm{Mg}^{2+}$ influx. Cross-link dependent structural changes of the resulting actin gels were observed. The morphology of the resulting network gel was found to be strongly dependent on the temperature as the gels collapsed to bundles by cooling down below $15{ }^{\circ} \mathrm{C}$. Furthermore, this process was shown to be partially reversible and the transition between different morphologies was also dependent on the vesicle size: while F-actin/ $\alpha$-actinin networks showed large filament rings in small GUVs, with a diameter below $12 \mu \mathrm{m}$, they demonstrated spider web-like structures in larger GUVs. These simplistic approaches highlight the interplay of F-actin and the membrane but the systems lack a direct and strong coupling to the lipid bilayer like it was shown for the 
plasma membrane. Besides the already presented membrane-cytoskeleton linker proteins also transmembrane proteins like ponticulin can serve as a direct linker.[70]

Direct coupling of the F-actin network inside a GUV was realized by introducing His 10 -tagged VVCA, an actin binding domain of the WASP protein family. ${ }^{[71,72]}$ This domain interacts with the Arp2/3 complex promoting the formation of a highly branched, dense network within the GUV. ${ }^{40]}$ Specific attachment was shown, because leaving out VVCA reduced the number of GUVs carrying an F-actin shell. The resulting F-actin cortex within the GUV had a thickness of about 100-400 nm and a mesh size of $150 \mathrm{~nm}$ demonstrating a good mimic of cellular cortices.

Furthermore, it was shown that the F-actin-membrane coupling affects the morphology of the resulting F-actin networks. Liu et al. showed that a strong F-actin network binding via the protein N-WASP to DOGS-Ni-NTA or PtdIns $[4,5] \mathrm{P}_{2}$ led to the formation of protrusions using an Arp2/3 controlled actin polymerization. ${ }^{[73]}$ The interaction of the growing, attached actin filaments with the membrane recruits further filaments, thus forming filopodia-like protrusions. Increasing Arp2/3 concentration decreased the number of protrusions, since the branching hindered the recruitment of further filaments within one protrusion. The formation of the bundled protrusion is obviously competing with the membrane's bending rigidity, as above a critical length of a protrusion it is energetically favourable to bundle filaments within different protrusions in order to minimize membrane tension. This shows that MAC based approaches enable to clear up part of the complex dynamic processes within the cellular actin cortex that control shape changes of cells.

\section{MACs outside of vesicles or droplets}

Forming an actin cortex inside a cell-sized vesicles is advantageous, as the vesicle size can adjust the morphology of the F-actin network like the situation is for actin cortices in cells. But actin cortices which are attached to the outside of a vesicle or droplet, like a shell, can overcome spatial constraints (cf. Figure 1.7 B) generating a different point of view. Furthermore, for reshaping the lipid bilayer by F-actin arrangement a strong binding between lipid bilayer and actin network is required. On the other hand, when binding of the F-actin network to the membrane is strong enough, the interfacial spatial dimensions still provide constraints. Additionally, the accessibility of these MACs is of course larger than MACs inside of vesicles or droplets.

Van der Gucht and co-workers studied the mechanics of actomyosin networks on shell-like structures using oil-in-water droplets. ${ }^{[4]}$ By means of particle tracking microrheology they showed that the amount of actin-membrane cross-linking determines the stiffness of a system and that an active, myosin induced rearrangement of the actin network results in further stiffening. A better mimic of the biological 
situation is created by using GUVs, which also allow to study the membrane-cortex interplay. ${ }^{[75-77]}$ For membrane coupling biotinylated G-actin monomers were introduced, which can bind streptavidin-mediated to biotinylated lipids on the outside of the GUV. It was found that the actin shell on the GUV leads to a stabilized shape of the vesicle. It must be considered that 2 biotinylated G-actin monomers can also be cross-linked by a streptavidin. Furthermore, the bending rigidity of the vesicular membrane was considerably increased and the vesicles showed viscoelastic behaviour in contrast to pure lipid vesicles which behave fully elastic. ${ }^{78]}$ The strong actin-membrane coupling is apparently required to mimic native cellular cortex' behaviour like bending stiffness and viscoelasticity.

The importance of network dynamics was demonstrated by Caorsi et al., who observed no change in bending rigidity and membrane tension when a pre-formed actin network was attached to cell-sized liposome doublets, but membrane deformation and an increase in tension when an initiated, dynamic polymerization, induced by activation of the Arp2/3 complex with S-pVCA, occurred. ${ }^{[9]}$ Membrane attachment was again realized using biotinylated lipids and G-actin monomers in conjunction with streptavidin. The direct influence on the membrane tension could be increased further by adding myosin motor proteins, which also led to an altered morphology of the strongly coupled network. Hence, for mimicking processes of cellular cortices it is required that the actin network is sufficiently dynamic.

Using MAC outside of GUVs, studies about phase separation phenomena of lipid mixtures of DPPC, DOPC and cholesterol doped with PtdIns [4,5] $\mathrm{P}_{2}$, to which the actin network was coupled, were performed. ${ }^{[80]}$ The connection between lipid bilayer and F-actin network was established by $\operatorname{PtdIns}[4,5] \mathrm{P}_{2}$ and N-WASP mimicking a physiological situation also found in cells. ${ }^{[71,81]}$ A dense network outside the GUV was achieved by Arp2/3 steered polymerization. It was reported that the miscibility temperature was higher when an actin shell was coupled to the GUV. Furthermore, using a higher PtdIns[4,5] $\mathrm{P}_{2}$ ratio and hence increasing the attachment points of the lipid bilayer and the actin shell also influenced the miscibility temperature indicating that a coupled actin network can stabilize phase separation in lipid bilayers.

Interestingly, when biotinylated lipids where designed to separate preferentially into the liquid-disordered phase, domain formation occurred along the actin filaments. Otherwise, a lipid anchor partitioning into the liquid-ordered phase favoured still, but less pronounced, an orientation along the filaments. These findings suggest that the cellular membrane composition is actively tuned by the dynamics of the attached cortex. ${ }^{[80]}$ 


\section{MACs on planar lipid bilayers}

While three-dimensional MAC systems allow the determination of mechanical properties and effects of compartmentation, small scale effects on the size of single filaments can be more effectively studied in planar, two-dimensional systems (Figure 1.7 C). Like spherical MACs, coupling between the F-actin network and membrane was found to significantly influence the properties of the MAC.

Schwille and co-workers presented an MAC coupled to a planar lipid bilayer via biotin-neutravidin binding. ${ }^{[82,83]}$ By adding myosin II motor proteins to the MAC they showed that contractile action of the actin network resulted in buckling and fragmentation of the filaments. The interaction of myosin and actin is strongly influenced by the coupling strength to the lipid bilayer. The established connection via biotin-neutravidin (or streptavidin) is known to be very strong compared to coupling in vivo, where PtdIns [4,5] $\mathrm{P}_{2}$ enables binding to ERM or WASP proteins. ${ }^{[55,71,84-88]}$ It is very likely that this strong coupling and the reduced mobility of the lipids in contrast to free-standing membranes, such as GUVs, led to fragmentation of F-actin induced by myosin. This indicates that a more physiological coupling of membrane and F-actin network is needed to mimic the situation in vivo.

The effect on the F-actin rearrangement of cross-linkers and motor proteins was further investigated in dependence of the coupling strength using MACs on planar membranes. ${ }^{[89,90]}$ Weakly attached MACs were achieved by crowding F-actin to the surface via methylcellulose and strong binding to the membrane was mediated by a FimA2-domain containing a His-tag, which can bind to DOGS-Ni-NTA lipids embedded in the solid supported membrane.[91] The resulting cortex was roughly $200 \mathrm{~nm}$ thick and was used to investigate the influence of $\alpha$-actinin and the contractile strain induced by myosin motor proteins. ${ }^{[92-94]}$ For weakly coupled networks, long-range rearrangement induced by myosin of the membrane coupled cortex was only observed in presence of $\alpha$-actinin and the contractile motor was strongly increased in contrast to experiments with only myosin motors. Otherwise, strong membrane-network coupling reduced the characteristic length scale of myosin's contractile motion. The underlying mechanism of active, in plane contraction by myosin was identified to be filament sliding. [95]

A combined linker setup was used by Köster et al., who used a linker consisting of the actin binding domain of ezrin (cf. chapter 1.3.1) and on the other side, a His-tag to bind DOGS-Ni-NTA within an artificial bilayer. ${ }^{[66]}$ This setup mimics the binding of ezrin to F-actin and benefits from the strong binding of the His-tag Ni-NTA interaction. ${ }^{[84]}$ The mobility of F-actin networks after myosin addition was studied, while the thickness of the cortex was controlled by actin concentration and filament length was adjusted by a capping protein. The dynamic formation of filament bundles, 
linked apolar asters and polar asters was observed, showing the same possibilities for network reshaping as vesicular model systems.

Another physiological, biomimetic approach to couple F-actin networks to artificial lipid bilayers is based on the transmembrane protein ponticulin. ${ }^{96]}$ Ponticulin containing solid supported lipid bilayers were incubated with F-actin to form a 20-nm thick, dense network layer on top. Taking advantage of the high affinity coupling between protein and actin network, atomic force micrographs were recorded, revealing a dense network covering $13.7 \%$ of the bilayer surface when roughly 250 ponticulin proteins per square micron are present in the bilayer. ${ }^{[70]}$ 


\section{Scope of Thesis}

The actin cortex is one of the most complex and dynamic network systems found in cells. The interplay of actin filaments and its over 100 accessory proteins are responsible for shape, stability and further functions of the cell. In that context the connection to the plasma membrane plays a pivotal role, which is realized by proteins of the ERM (ezrin-radixin-moesin) family. In the vast number of actin binding proteins (ABP), it is highly challenging to characterize the function or the influence of single components contributing to the complex network.

Therefore, bottom-up approaches in artificial setups are used, to determine the specific features of single proteins to an ABP-free filamentous actin (F-actin) network. In this work, a minimal actin cortex (MAC), containing a lipid bilayer, an active mutant of ezrin and an F-actin network, will be created with the use of the physiological linker ezrin as its unique feature in contrast to setups reported in literature.

In a first step, the concentration of the receptor lipid L- $\alpha$-phosphatidylinositol4,5-bisphosphate (PtdIns[4,5] $\left.\mathrm{P}_{2}\right)$, serving as pinning points for ezrin, will be varied, thus changing the surface coverage of ezrin and providing different amounts of connection points for the F-actin network. The organization of the F-actin on the lipid bilayer, dependent on the pinning point density, will be investigated by means of confocal fluorescence microscopy (CLSM) in order to characterize the influence of ezrin on the MAC formation. The MAC will be characterized by height, filament lengths and network density.

In a second step, the change of the viscoelastic properties of membrane attached F-actin networks in contrast to $3 \mathrm{D}$ F-actin gels will be investigated by means of video particle tracking microrheology. Furthermore, the F-actin induced change in lateral membrane tension will be studied by indentation experiments. Both experimental setups will provide information about the change in the mechanical properties of the F-actin network, as well as the lipid bilayer, in case of association between both.

In a last step, the created MAC will be used as a tool to characterize the influence of single actin binding proteins on the actin cortex. The impact of two different cross-linking proteins will be analyzed, fascin and $\alpha$-actinin. This setup will provide information about the functionalities of those proteins, which can be related to their localizations and functions in vivo. 


\section{Materials and Methods}

\subsection{Used Materials}

\subsubsection{Lipids}

\subsubsection{Matrix Lipids}

Artificial lipid bilayers are set up with two different types of lipids. The first type is designated as matrix lipids. This specification refers to those lipids which represent the larger portion but should have no significant effect to the attached proteins. Unwanted matrix effects can appear and must be disproved in blind experiments. Experiments of this work entail 1,2-dioleoyl-sn-glycero-3-phosphocholine (DOPC) and 1-palmitoyl2-oleoyl-sn-glycero-phosphocholine (POPC, see Table 3.1). Both lipids provide no charge and a similar structural framework. Glycerol is substituted with a phosphocholine head group at position 3 and esterified with an oleic acid (18:1 $\Delta^{9}$-cis) on position 2. Whereas DOPC carries a second oleic acid at position 1, POPC is esterified with a palmitic acid (16:0). Due to more cis double bonds the fluidity of DOPC is slightly higher than the fluidity of POPC.[97] All lipids were purchased by Avanti Polar Lipids (Alabaster, AL, USA).

Table 3.1: Structural framework and molecular masses of matrix lipids.

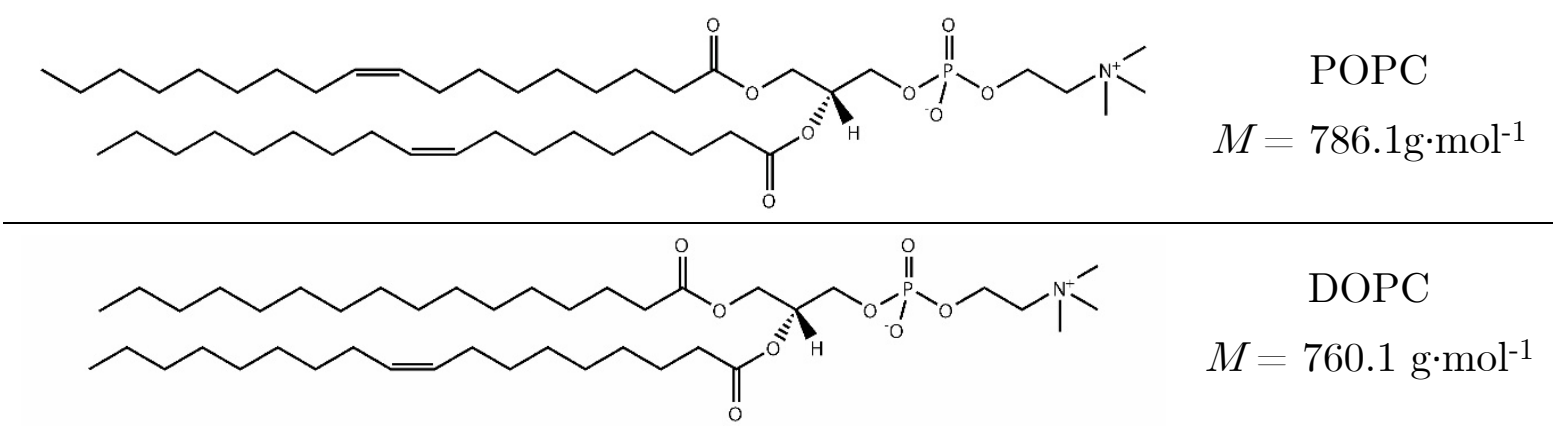

\subsubsection{Receptor Lipids}

Besides matrix lipids an artificial membrane consists of 'active' parts: lipids that are intended to affect proteins or other material are designated as receptor lipids. In this work the physiologically relevant L- $\alpha$-phosphatidylinositol-4,5-bisphosphate (PtdIns $[4,5] \mathrm{P}_{2}$ ) was mostly used. The specific phosphate head group is negatively charged and known to interact in many signaling pathways (see chapter 1.3.1). PtdIns $[4,5] \mathrm{P}_{2}$ was purchased as natural lipid extracted from porcine brain with a 
determined fatty acid distribution (Avanti, Alabaster, AL, USA). ${ }^{[98]}$ In Table 3.2 the most frequent type and an average molecular mass $\left(M_{\mathrm{av}}\right)$ is given. Furthermore, another protein binding lipid 1,2-dioleoyl-sn-glycero-3-phosphoethanolamine- $N$-(cap biotinyl) (cap-bio-DOPE) was used. The biotin head is known to bind specifically to avidin. All lipids were purchased by Avanti Polar Lipids (Alabaster, AL, USA).

Table 3.2: Structural framework and molecular masses of receptor lipids.

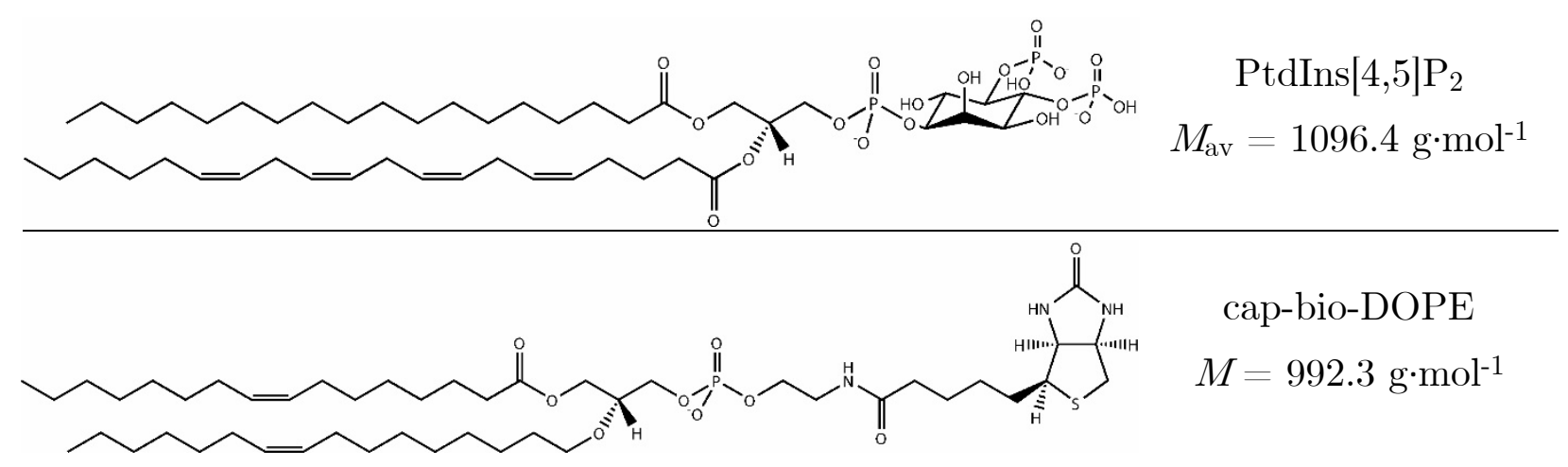

\subsubsection{Positively Charged Lipids}

F-actin networks carry a negative charge, so they can attach to positively charged lipid layers. For this purpose, 1,2-dioleoyl-sn-glycero-3-ethylphosphocholine (DOEPC), $3 ß-\left[N-\left(N^{\prime}, N^{\prime}\right.\right.$-dimethylaminoethane)-carbamoyl]cholesterol (DC-Chol) and $N$-[1-(2,3-dioleoyloxy)propyl]- $N, N, N$-trimethylammonium (DOTAP) were used. Whereas DOEPC has a very similar structural framework to the used matrix lipids with only the difference of the added ethyl-group (see chapter 3.1.1.1) DC-Chol and DOTAP differ more clearly to them. DC-Chol is a derivate of cholesterol, which is esterified with a dimethylaminoethane carbamic acid at 3-hydroxy group to insert a positive charge (see Table 3.3). Like cholesterol does, DC-Chol can also modulate the fluidity of the membrane. While the fluidity effect remains similar to cholesterol DC-Chol forms less domains in the lipid bilayer.[99] On the contrary, the positively charged lipid DOTAP consists of the same fatty acids like DOPC does but differs in the head group. It is no phospholipid but has a trimetylammonium-propane group as positively charged head and is already widely used in literature.[100-102] Furthermore, studies with DOTAP binding to F-actin already showed the functionality of the desired effect which will be pursued in this work. ${ }^{[103]}$ All lipids were purchased by Avanti Polar Lipids (Alabaster, AL, USA). 
Table 3.3: Structural framework and molecular masses of positively charged lipids.

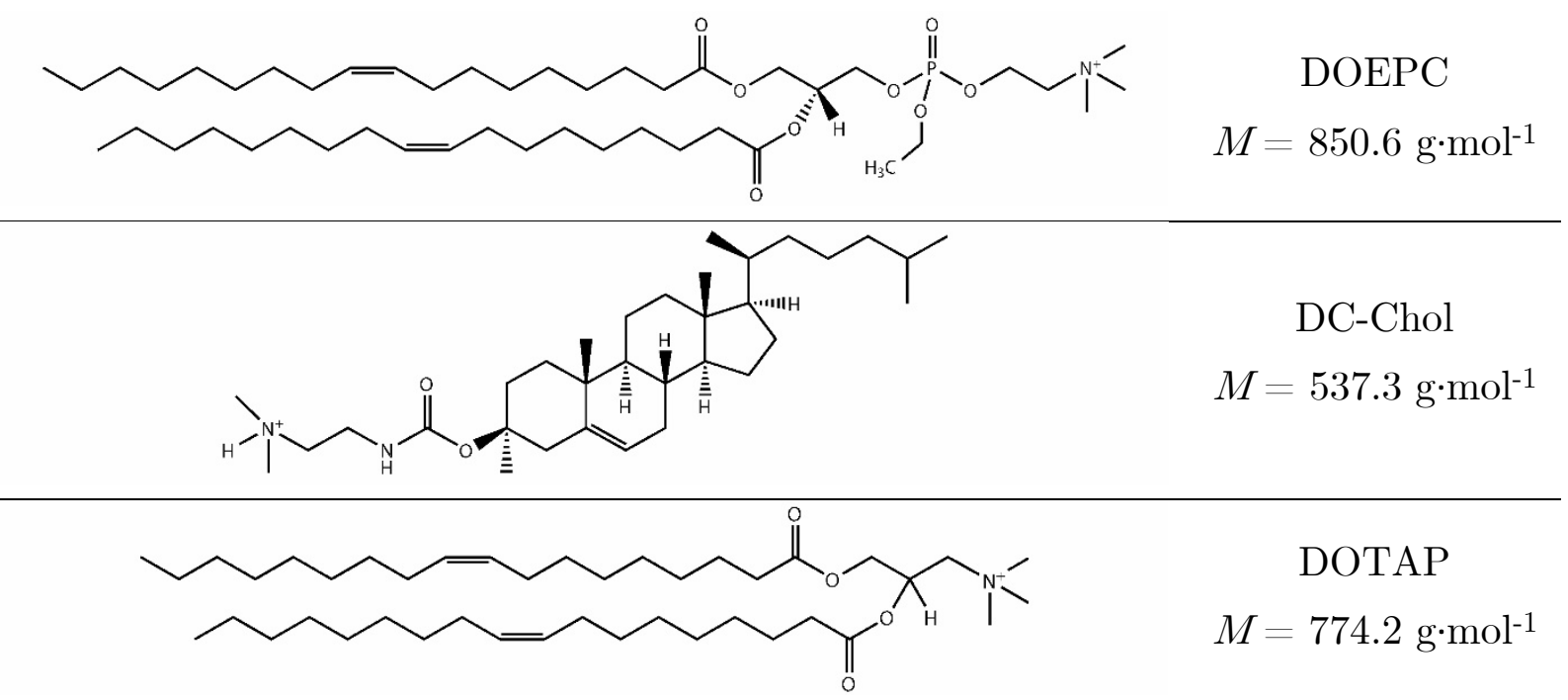

\subsubsection{Fluorophores}

\section{Texas $\operatorname{Red}^{\circledR}(\operatorname{TxR})$}

The fluorophore Texas $\operatorname{Red}^{\circledR}$ (TxR) which is chemically a sulforhodamine 101 acid chloride was used in this work as label for artificial membranes. Therefore, a lipid bound fluorophore (more precisely head group bound) TxR-DHPE, TxR 1,2dihexadecanoyl-sn-glycero-3-phosphoethanolamine, with a molar mass of $M=1381.9 \mathrm{~g} \cdot \mathrm{mol}^{-1}$ was used to detect lipid layers (Scheme 3.1 ). The red dye was excited at $\lambda_{\mathrm{ex}}=561 \mathrm{~nm}$ and detected at 580-640 nm. TxR-DHPE was procured from Thermo Fisher Scientific, Waltham, MA, USA.

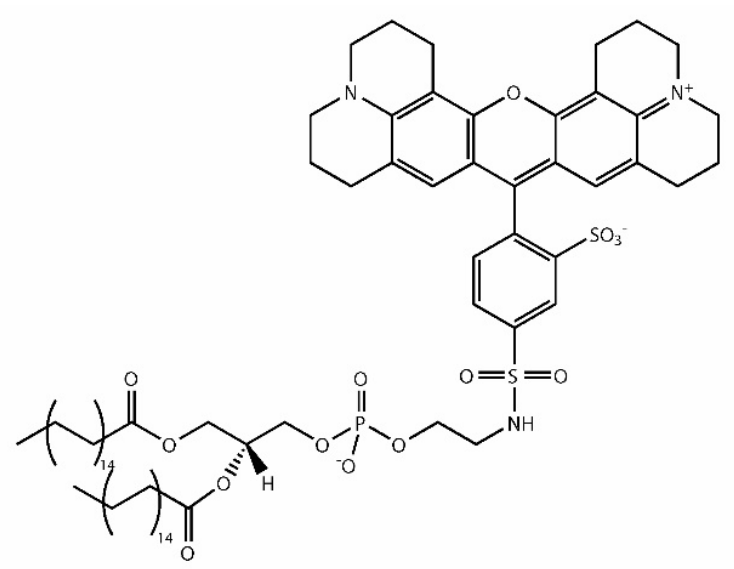

Scheme 3.1: Structural framework of TxR-DHPE.

\section{Atto 488}


Atto 488 is a green fluorescent label developed by Atto-Tec GmbH, Siegen, Germany. In this work an Atto 488-maleimide conjugate (Scheme 3.2) with a molar mass of $M=1067.0 \mathrm{~g} \cdot \mathrm{mol}^{-1}$ was used for protein labeling (chapter 3.2.7). Atto 488 was excited at $\lambda_{\mathrm{ex}}=488 \mathrm{~nm}$ and detected at $500-545 \mathrm{~nm}$.

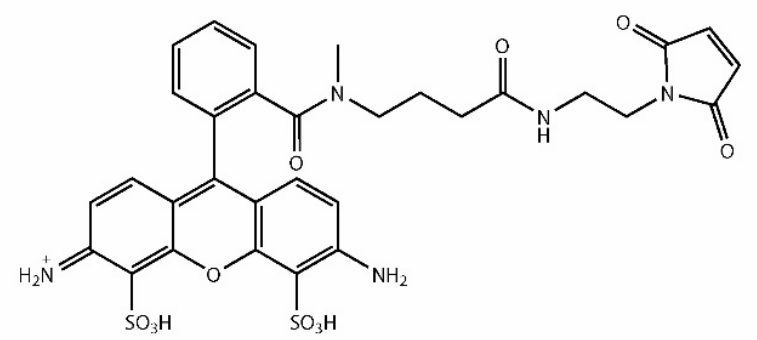

Scheme 3.2: Structural framework of Atto 488-maleimide.

\section{Alexa Fluor ${ }^{\circledR} 488$}

Alexa Fluor ${ }^{\circledR} 488$ is a dye out of the family of fluorescent dyes called Alexa Fluor by Thermo Fisher Scientific, Waltham, MA, USA. Like the already described dye Atto 488 , this dye was excited at $\lambda_{\mathrm{ex}}=488 \mathrm{~nm}$ and detected at $500-545 \mathrm{~nm}$. In this work a conjugate of Alexa Fluor ${ }^{\circledR} 488$ and phalloidin (chapter 1.3) with a molecular mass of $M=1069 \mathrm{~g} \cdot \mathrm{mol}^{-1}$ as shown in Scheme 3.3 was used to label filamentous actin.

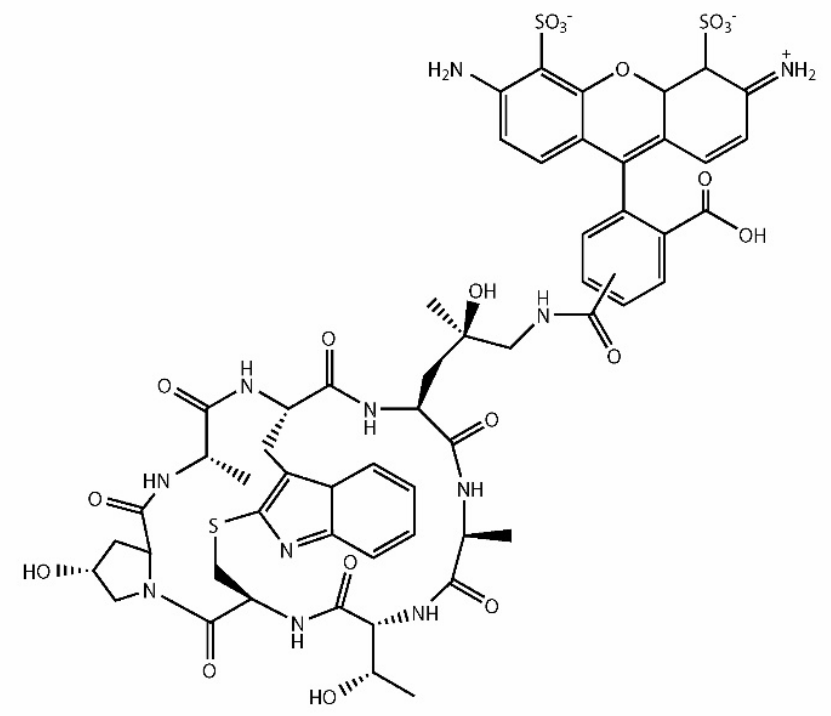

Scheme 3.3: Structural framework of Alexa Fluor ${ }^{\circledR}$ 488-phalloidin.

\section{Atto 390}

In case of microrheology experiments (chapter 3.6.4) another dye had to be used because tracer particles were labeled in red and F-actin was already labeled with green dye. Therefore, the membrane had to be labeled in another color which was overcome by the usage of the blue, lipid head group bound fluorophore Atto 390-DOPE (Scheme 
3.4) with the molecular mass of $M=1069 \mathrm{~g} \cdot \mathrm{mol}^{-1}$. Atto 390 is also a product from Atto-Tec GmbH, Siegen, Germany.

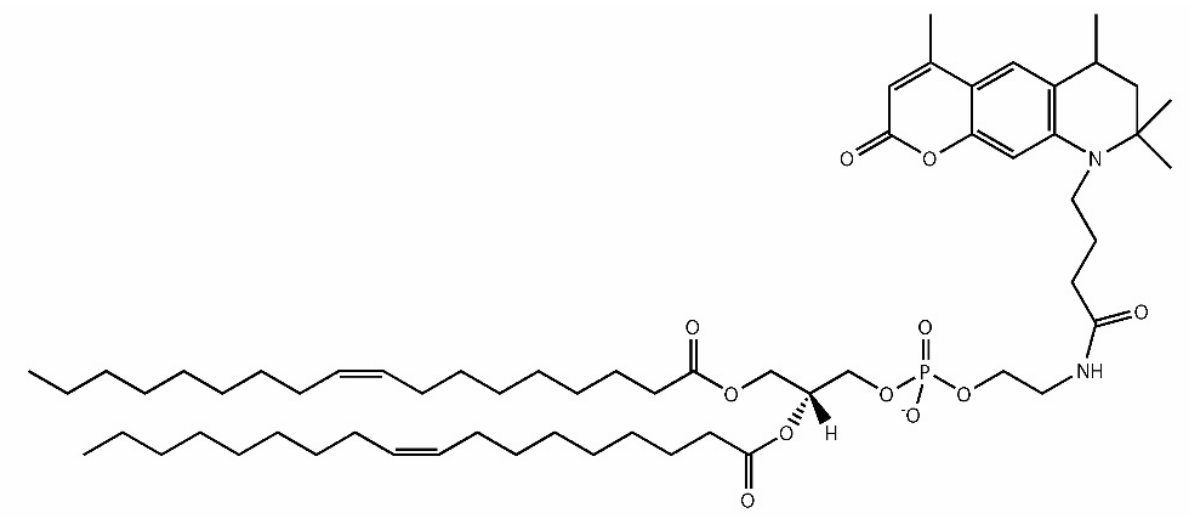

Scheme 3.4: Structural framework of Atto 390-DOPE. 


\subsection{Biochemical Methods}

\subsubsection{Plasmid Transformation}

A pET28a + vector (Novagen, Madison, WI) containing the decoding information was transformed into E. coli (Escherichia coli) cells via heat shock. The decoding information or gene of interest is located between a $\mathrm{T} 7$ promoter and a $\mathrm{T} 7$ terminator sequence and can therefore be decoded by the T7 RNA polymerase. Further noteworthy sequences are the kan coding sequence responsible for the Kanamycin resistance and lacI repressor sequence which ensures a inhibition of the lac operon. [104] A schematic layout of the plasmid is included in the appendix (chapter 7.1). E. coli cells of the strain BL21(DE3)pLysS were purchased by Invitrogen, Carlsbad, CA, USA.

\section{Experimental procedure}

$4 \mathrm{\mu L}$ of the plasmid solved in ultrapure $\mathrm{H}_{2} \mathrm{O}\left(c=166 \mathrm{ng} \cdot \mathrm{\mu L}^{-1}\right)$ were pipetted to one tube containing component cells of the strain BL21(DE3)pLysS $(50 \mu \mathrm{L})$ and incubated for 30 minutes. By placing the cells for 30 seconds into a $42{ }^{\circ} \mathrm{C}$ water bath the heat shock was applied. Subsequently the cells were placed on ice for 4 minutes for regeneration. After addition of $250 \mu \mathrm{L}$ SOC-medium (super optimal broth with catabolite repression) the cell culture was incubated at $37^{\circ} \mathrm{C}$ in a shaker for one hour. A selective growth was guaranteed by placing the culture on a LB (lysogeny broth) agar plate with added antibiotics (Kanamycin, Chloramphenicol, in each case $c=0.1 \mathrm{mM}$ ) overnight. After successful plasmid transformation single-colonies were grown on the agar plate as shown in Figure 3.1.

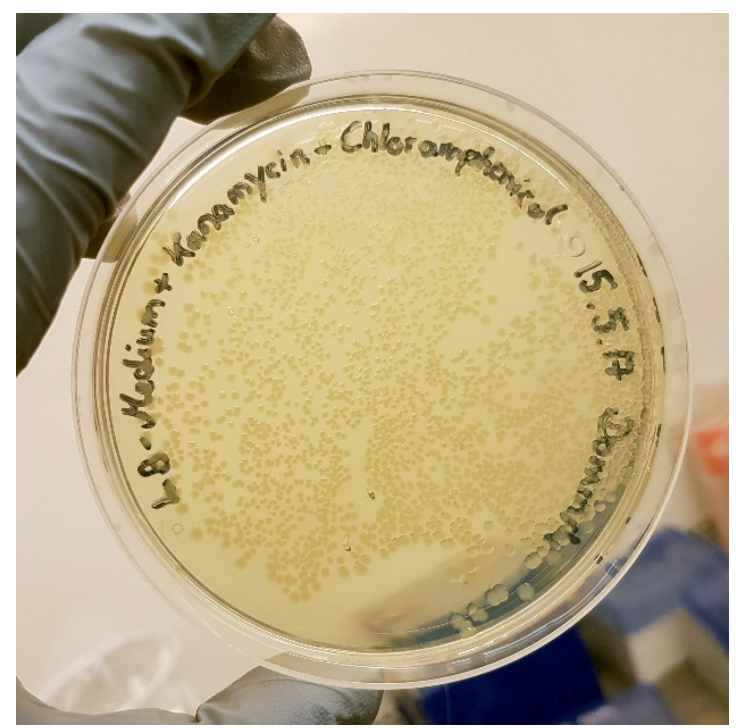

Figure 3.1: Single-colonies grown on an agar plate after successful transformation. An agar plate of LB medium with added antibiotics (Kanamycin, Chloramphenicol) served as nutrition for transformed E. coli cells. Since only successful transformed cells carry the Kanamycin resistance, solely these desired colonies can grow on the nutrient medium. 


\begin{tabular}{|c|c|c|c|}
\hline LB-agar & $\begin{array}{l}1.5 \%(\mathrm{~W} / \mathrm{V}) \text { agar } \\
1.0 \%(\mathrm{~W} / \mathrm{V}) \text { tryptone } \\
0.5 \%(\mathrm{~W} / \mathrm{V}) \text { yeast extract } \\
10 \mathrm{mM} \mathrm{NaCl} \\
\mathrm{pH} 7.4\end{array}$ & SOC-medium & $\begin{array}{l}2.0 \%(\mathrm{~W} / \mathrm{v}) \text { tryptone } \\
0.5 \%(\mathrm{~W} / \mathrm{v}) \text { yeast extract } \\
10 \mathrm{mM} \mathrm{NaCl} \\
2.5 \mathrm{mM} \mathrm{KCl} \\
10 \mathrm{mM} \mathrm{MgCl}_{2} \\
10 \mathrm{mM} \mathrm{MgSO}_{4} \\
20 \mathrm{mM} \mathrm{glucose}^{\mathrm{pH} 7.0}\end{array}$ \\
\hline
\end{tabular}

\subsubsection{Plasmid Isolation}

Plasmid isolation was performed using a Necleobond ${ }^{\circledR}$ Xtra Midi EF-Kit (Machery-Nagel, Düren, Germany).[105] First, $100 \mathrm{~mL}$ LB medium was inoculated by picking a single colony grown on an agar plate as described in chapter 3.2.1 (Figure 3.1 ) at $37{ }^{\circ} \mathrm{C}$ overnight. Grown bacteria culture was centrifuged (4500 rpm, $20 \mathrm{~min}$, $4{ }^{\circ} \mathrm{C}$ ) and the resulting pellet was resuspended with $8 \mathrm{~mL}$ RES-EF buffer including RNase A. An alkaline cell lysis was performed by adding $8 \mathrm{~mL}$ LYS-EF buffer followed by a five-minute incubation at room temperature. A silica-based anion-exchange column was equilibrated with $15 \mathrm{~mL}$ EQU-EF buffer while $8 \mathrm{~mL}$ NEU-EF buffer was added to the lysate and incubated on ice for $5 \mathrm{~min}$. After incubation cell fragments were separated from lysate by centrifugation (4500 rpm, $10 \mathrm{~min}, 20{ }^{\circ} \mathrm{C}$ ). The lysate was then added to the equilibrated column and subsequently washed with $5 \mathrm{~mL}$ FIL-EF buffer, $35 \mathrm{~mL}$ ENDO-EF buffer and $15 \mathrm{~mL}$ WASH-EF buffer. Washed plasmid was eluted by $5 \mathrm{~mL}$ ELU-EF buffer. For full purification $3.5 \mathrm{~mL}$ isopropyl alcohol was added to the plasmid and the suspension was centrifuged again (4500 rpm, $45 \mathrm{~min}$, $4{ }^{\circ} \mathrm{C}$ ). Resulting supernatant was removed and the pellet dissolved in endotoxin-free ethanol. Centrifugation (13000 rpm, $5 \mathrm{~min}, 20^{\circ} \mathrm{C}$ ) and solvent removing was repeated, and the remaining pellet was shaken in a thermomixer at $37^{\circ} \mathrm{C}$ for one hour to remove remaining solvent. Plasmid was collected in $1.2 \mathrm{~mL}$ ultrapure water to obtain the desired plasmid solution. This solution was stored at $-21{ }^{\circ} \mathrm{C}$ until use. Purchased buffers from the kit were used as described above, a detailed composition was not given by the manufacturer.[105]

\subsubsection{Plasmid Sequencing}

Plasmid sequencing was implemented by the company Seqlab, Inc. located in Göttingen. Sequencing was used to control the plasmid beyond time to check that there were no mutations. Primers (T7 promotor and T7 terminator) were directly provided by Seqlab. 


\subsubsection{Protein Expression}

Recombinant protein expression of ezrin T567D with an $N$-terminal hexahistidine-tag (His ${ }_{6}$-tag) was implemented following a prescription from earlier studies. ${ }^{[106]} \mathrm{A}$ successful transformed single-colony (cf. chapter 3.2.1) of E. coli bacteria (strain BL21(DE3)pLysS) carrying the pET28a + with desired protein decoding information was picked and induced to a $10 \mathrm{~mL}$ pre-culture of LB medium with added antibiotics (Kanamycin, Chloramphenicol, in each case $c=0.1 \mathrm{mM}$ ). Pre-culture was allowed to grow while shaking at $37{ }^{\circ} \mathrm{C}$ overnight. Protein main culture (LB medium with $0.1 \mathrm{mM}$ Kanamycin) was induced by adding $2 \%(\mathrm{~V} / \mathrm{v})$ of the grown pre-culture. This procedure was necessary to monitor the bacterial growth precisely. Bacterial cell culture grew till an optical density $\left(\mathrm{OD}_{600}\right)$, measured at a wavelength of $600 \mathrm{~nm}$, of 0.6 was achieved and subsequently protein expression was induced by adding $1 \mathrm{mM}$ isopropyl $\beta$-D-thioglactopyranoside (IPTG). After 4 hours of protein expression at $37^{\circ} \mathrm{C}$ while shaking, bacterial culture was placed on ice for $10 \mathrm{~min}$. Bacterial pellet was obtained by centrifugation (4500 rpm, $20 \mathrm{~min}, 4{ }^{\circ} \mathrm{C}$ ) and resuspended in lysis buffer including the protease inhibitor cocktail cOmplete ${ }^{\mathrm{TM}}$ (Roche diagnostics, Basel, Switzerland). Lysis was mechanically completed by tip sonification (Sonopuls, Bandelin electronic GmbH \& Co. KG, Berlin; $60 \%$ power, $5 \times 30 \mathrm{sec}$ ). Concluding centrifugation (33000 rpm, $1 \mathrm{~h}, 4{ }^{\circ} \mathrm{C}$ ) separated expressed protein from remaining cell fragments.

\begin{tabular}{|c|c|}
\hline LB-medium $1.0 \%(\mathrm{w} / \mathrm{V})$ tryptone & lysis buffer $300 \mathrm{mM} \mathrm{NaCl}$ \\
\hline $0.5 \%(\mathrm{~W} / \mathrm{v})$ yeast extract & 40 mM HEPES \\
\hline $10 \mathrm{mM} \mathrm{NaCl}$ & $20 \mathrm{mM}$ imidazole \\
\hline pH 7.4 & $1 \mathrm{mM}$ EDTA \\
\hline & $10 \mathrm{mM} \beta$-mercaptoethanol* \\
\hline & $\mathrm{pH} 7.4$ \\
\hline & $*_{\text {immediately before use }}$ \\
\hline
\end{tabular}

\subsubsection{Protein Purification}

Purification of the protein ezrin T567D was conducted after the principle of immobilized metal ion affinity chromatography using the $N$-terminal added His $_{6}$-tag. ${ }^{[107,108]}$ This His $_{6}$-tag is able to bind selectively to nickel cations, which are provided by the metal chelating nickel-nitrilotriacetic acid (Ni-NTA) agarose ( Quiagen, Hilden, Germany) as shown in Scheme 3.5. A consequent difference in binding affinities of the His $_{6}$-tag and imidazole to nickel is used to purify the protein. The Ni-NTA agarose is filled into a purification column and after settlement of the agarose suspension a column volume of around $2 \mathrm{~mL}$ was achieved. After washing with 
ultrapure water and equilibration buffer (EQ, each 10 column volumes), the lysate (supernatant) resulting from protein expression was applied three times to the Ni-NTA column running through by gravity flow. Procedure steps were employed at $4{ }^{\circ} \mathrm{C}$. The column-bound was washed utilizing 10 column volumes of each washing buffer (Wash I and Wash II) with increasing the imidazole concentration. Subsequently, elution was performed with 10 column volumes of elution buffer providing a particularly high imidazole concentration to collect purified protein from the column. Protein was collected in elution fractions of around $1.5 \mathrm{~mL}$. Lysate, each washing fraction and the elution fractions were analyzed by means of SDS-PAGE (cf. chapter 3.2.6).

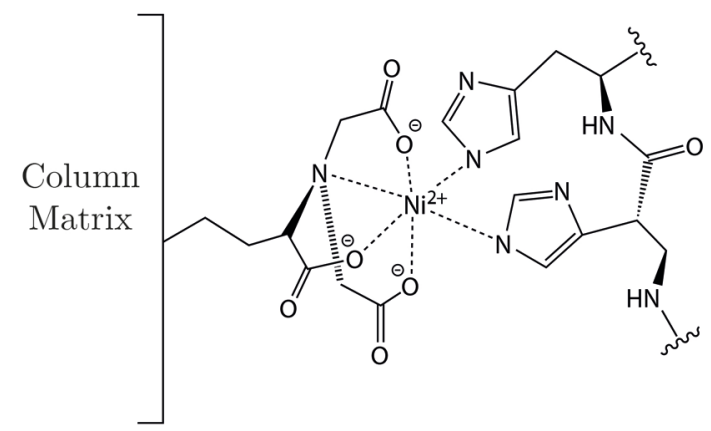

Scheme 3.5: Structural framework of Ni-NTA coordinatively binding nickel. The empty binding sites are used to bind the histidine residues from protein's His6-tag as illustrated above.

Elution fractions containing the desired protein in acceptable degree were combined and dialyzed three times ( $2 \times 4$ hours and 1 x overnight) against the 50 -fold volume of protein buffer (E1 buffer). Protein was stored at $4{ }^{\circ} \mathrm{C}$ for a maximum of 12 weeks.

EQ buffer $300 \mathrm{mM} \mathrm{NaCl}$

$40 \mathrm{mM}$ HEPES

$20 \mathrm{mM}$ imidazole

$\mathrm{pH} 7.4$ wash I

$300 \mathrm{mM} \mathrm{NaCl}$

40 mM HEPES

$30 \mathrm{mM}$ imidazole

$1 \mathrm{mM} \mathrm{MgCl} 2$

$10 \mathrm{mM} \beta$-mercaptoethanol*

pH 7.4 wash II $\quad 150 \mathrm{mM} \mathrm{NaCl}$

$40 \mathrm{mM}$ HEPES

$40 \mathrm{mM}$ imidazole

$1 \mathrm{mM} \mathrm{MgCl}{ }_{2}$

$10 \mathrm{mM} \beta$-mercaptoethanol*

pH 7.4 elution buffer

$50 \mathrm{mM} \mathrm{KCl}$

$20 \mathrm{mM}$ Tris $/ \mathrm{HCl}$

$250 \mathrm{mM}$ imidazole

pH 7.4

*immediately before use 


\section{E1 buffer $50 \mathrm{mM} \mathrm{KCl}$ \\ $20 \mathrm{mM}$ Tris/HCl \\ $0.1 \mathrm{mM}$ EDTA \\ $0.1 \mathrm{mM} \mathrm{NaN}_{3}$ \\ $\mathrm{pH} 7.4$}

\subsubsection{Sodium Dodecyl Sulfate Polyacrylamide Gel Electrophoresis (SDS-PAGE)}

A sodium dodecyl sulfate polyacrylamide gel electrophoresis (SDS-PAGE) is a useful technique to prove the quality and size of proteins originally published by U.K. Laemmli.[109] It separates proteins by their electrophoretic mobility by using a polyacrylamide gel separated into a stacking and a resolving gel. The detergent SDS is denaturing proteins by disrupting non-covalent interactions and thus itself binding the protein non-covalently masking the proteins intrinsic charges. Therefore, it supplies a negative charge according to the molecular weight of the protein so that a separation by their ratio of charge-to-mass becomes possible. Besides charge-to-mass ratio the pore size of the gel is an important factor of SDS-PAGE.[109] The gel is separated into a stacking gel (5.2\%, pH 8.8) which collects the protein and a resolving gel $(12.5 \%, \mathrm{pH} 6.8)$ which finally separates proteins. The gel is produced by radical polymerization of acrylamide and bisacrylamide.

\section{Experimental procedure}

The vertical gel electrophoresis kit 'Mini-PROTEAN ${ }^{\circledR}$ Tetra Cell' retrieved from Bio-Rad Laboratories GmbH (München, Germany) was used in this work. First, glass chambers were cleaned with ultrapure water and ethanol p.a. and subsequently resolving gel stock solution (cf. after prescription) was filled into these chambers. Stock solution has previously been combined with the polymerization initiator ammonium peroxydisulfate (APS, $0.1 \%(\mathrm{~W} / \mathrm{V})$ ) and the catalyst $N, N, N^{\prime}, N^{\prime}$-tetramethyl ethylenediamine (TEMED, $V=2 \mu \mathrm{L}$ ). This polymerization solution was filled up to two-thirds and was covered with isopropanol to guarantee a straight transition line. After 1 hour of polymerization isopropanol was removed and the stacking gel stock solution again combined with APS $(0.1 \%(w / v))$ and TEMED $(V=2 \mu \mathrm{L})$ was poured onto the resolving gel. A comb defining the specimen pockets was immediately placed into the top of the stacking gel which was then allowed to polymerize for 1 hour. After polymerization the comb was removed, and the electrophoresis chamber was filled with running buffer. $10 \mu \mathrm{L}$ protein solution of each fraction (cf. chapter 3.2.5) was collected and merged with $10 \mu \mathrm{L}$ sample buffer. These merges were heated up in a thermomixer 
at $95{ }^{\circ} \mathrm{C}$ (350 rpm, $\left.5 \mathrm{~min}\right)$ and subsequently pipetted into the gel pockets. One pocket was filled with low molecular weight markers (GE Healthcare, Freiburg, Germany) and the whole chamber was connected to a power source applying $15 \mathrm{~mA}$ per gel. After a run of around 90 minutes the gels were washed with warm ultrapure water, heated up in a microwave $(800 \mathrm{~W}, 45 \mathrm{sec})$. Subsequently, the gel was stained with water-Coomassie solution which was heated up in a microwave ( $800 \mathrm{~W}, 45 \mathrm{sec}$ ) again. After two runs of decolorizing in warm ultrapure water (by microwave, $800 \mathrm{~W}, 45 \mathrm{sec}$ ) the gels were imaged with an Azure c300 gel imaging system (Azure Biosystems, Inc., Dublin, CA, USA).

resolving gel

$$
\begin{aligned}
& 12.5 \% \text { AA-mix* } \\
& 258 \text { mM Tris/HCl } \\
& 0.1 \%(w / v) \text { SDS } \\
& \text { pH } 6.8
\end{aligned}
$$

\author{
stacking gel $5.2 \%$ AA-mix* \\ $127 \mathrm{mM}$ Tris $/ \mathrm{HCl}$ \\ $0.1 \%(w / v)$ SDS \\ $\mathrm{pH} 8.8$
}

*AA-mix consists of acrylamide and bisacrylamide in the ratio of 37.5:1

running buffer

$25 \mathrm{mM}$ Tris $/ \mathrm{HCl}$

$192 \mathrm{mM}$ glycine

$0.1 \%(w / v)$ SDS

$\mathrm{pH} 8.3$ sample buffer $125 \mathrm{mM}$ Tris $/ \mathrm{HCl}$
(2x) $100 \mathrm{mM}$ DTT
$20 \%(\mathrm{~W} / \mathrm{V})$ glycerol
$2 \%(w / v)$ SDS
$0.02 \%(\mathrm{~W} / \mathrm{V})$ bromophenol blue $\mathrm{pH} 6.8$

water-Coomassie solution

$0.08 \%$ o $(w / v)$ Coomassie Brilliant Blue G-250 $25 \mathrm{mM} \mathrm{HCl}$ molecular weight markers
97 kDa phosphorylase B

$66 \mathrm{kDa}$ albumin

$45 \mathrm{kDa}$ ovalbumin

$30 \mathrm{kDa}$ carbonic anhydrase

$20.1 \mathrm{kDa}$ trypsin inhibitor

$14.4 \mathrm{kDa} \alpha$-lactalbumin

\subsubsection{Protein Labeling}

Labeling is a versatile tool to visualize proteins. A common approach is the chemical modification of cysteine residues using the thiol reactivity.[110,111] Besides the thiol 
reactivity the relatively rarity throughout the proteome is also a huge advantage. ${ }^{[110]}$ As modification a thiol-maleimide Michael addition is used because the quite mild reaction conditions are necessary in case of proteins as reactant. A slightly alkaline pH is needed due to the reason the thiol-maleimide Michael addition is alkaline catalyzed. Furthermore, proteins form selectively disulfide bridges thus reductive reaction conditions are necessary and will be achieved by using dithiothreitol (DTT).[111] In case of ezrin T567D two cysteine residues at C117 and C284 are present for labeling and Atto $488 \mathrm{C}_{5}$-maleimide is used as fluorescent compound. The mechanism of this thiol-maleimide Michael addition is illustrated in Scheme 3.6.

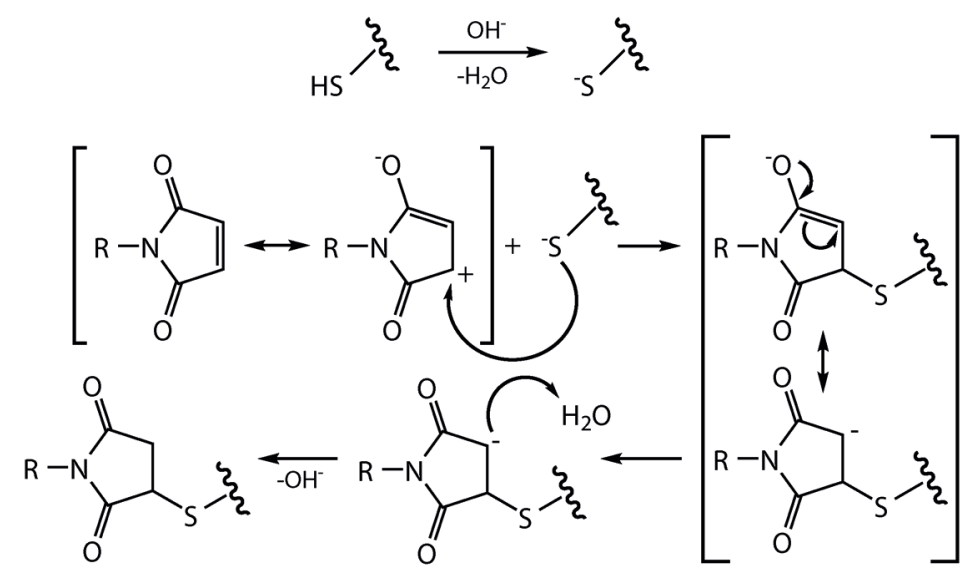

Scheme 3.6: Reaction mechanism of the thiol-maleimide-addition. The cysteine's thiol is deprotonated in an alkaline milieu and is attacking the carbon atom at the $\alpha$-position in a nucleophilic manner. The resulting carbanion attacks a water molecule which leads to the regenerated catalyst and the coupled product of maleimide and cysteine residue.

An alkaline catalyzed thiolate derived from the cysteine residue attacks nucleophilic the carbenium ion which is a resonance structure of the maleimide. The resulting carbanion attacks in turn nucleophilic a water molecule and regenerates the catalyst. The resulting product of the reaction is a coupled structure of the maleimide and the cysteine residue of the protein. The residue $R$ displays the attached fluorophore.

\section{Experimental procedure}

Ezrin T567D was concentrated $(c \geq 15 \mu \mathrm{M})$ by means of centrifugation using Vivaspin ${ }^{\circledR}$ 500 centricons (Sartorius AG, Göttingen, Germany). DTT was added to the protein solution in a 10 -fold molar excess and shook at $4{ }^{\circ} \mathrm{C}$ for 1 hour. Subsequently, the solution was dialyzed three times against $\mathrm{N}_{2}$-flushed $\mathrm{E} 1$ buffer necessarily free of oxygen.[110] Atto 488-maleimide was dissolved in ice cold ultrapure water $\left(c=10 \mathrm{mg} \cdot \mathrm{mL}^{-1}\right)$ and added in a 20 -fold molar excess and shook at $4{ }^{\circ} \mathrm{C}$ overnight. 
Remaining thiol reactive fluorophore was quenched with 40-fold molar excess of $\beta$-mercaptoethanol within 30 minutes. To separate free fluorophore from protein-bound fluorophore a size exclusion chromatography was applied. A Sephadex G-25 column (GE Healthcare, Freiburg, Germany) was equilibrated and protein solution was run through the column by gravity flow. Collected elution fractions were analyzed by means of UV/Vis spectroscopy (cf. chapter 3.4.2), fractions with fluorescent protein combined and concentrated again by means of centrifugation. 


\subsection{Preparative Methods}

Artificial lipid bilayers were formed on two different supporting materials to mimic the plasma membrane. On the one hand small unilamellar vesicles were spread on a continuous solid support to obtain a planar lipid bilayer. On the other hand, a porous substrate was used. Besides the solid supported parts, the lipid bilayer also spanned the porous regions to create free standing lipid bilayers. Therefore, giant unilamellar vesicles were used to overcome the pore size of the substrate.

\subsubsection{Preparation of Small Unilamellar Vesicles}

Small unilamellar vesicles (SUVs) have a diameter up to $100 \mathrm{~nm}$ and were obtained by sonification of rehydrated lipid films.

\section{Experimental procedure}

Lipid mixtures with the desired composition were first merged in a chloroform solution inside a test tube. In case of PtdIns $[4,5] \mathrm{P}_{2}$ methanol in a ratio of 1:10 to chloroform was added to the solvent. For this purpose, lipid stock solutions $\left(c \approx 1-11 \mathrm{mg} \cdot \mathrm{ml}^{-1}\right)$ were used which concentrations were determined via a Langmuir-Blodgett trough. Afterwards the solvent was dispersed with a nitrogen flush and dried under vacuum for at least three hours. These lipid films could be stored at $4{ }^{\circ} \mathrm{C}$ until required.

For rehydration $500 \mu \mathrm{L}$ of the desired buffer solutions (lipid-buffer combination shown in Table 3.4) were added to the test tube. After $30 \mathrm{~min}$ the suspension was vortexed three time in a five-minute interval. SUVs were finally gained via $30 \mathrm{~min}$ of sonification (cycle 4, $60 \%$, Bandelin, Berlin, Germany).

Table 3.4: Buffer solutions used in case of each receptor lipid.

\begin{tabular}{c|c} 
Receptor lipid & Buffer solution \\
\hline PtdIns $[4,5] \mathrm{P}_{2}$ & Na-citrate buffer \\
cap-bio DOPE & PBS buffer
\end{tabular}

Na-citrate buffer $50 \mathrm{mM} \mathrm{KCl}$

$20 \mathrm{mM}$ Na-citrate

$0.1 \mathrm{mM}$ EDTA

$0.1 \mathrm{mM} \mathrm{NaN}_{3}$

pH 4.8
PBS buffer

$2.7 \mathrm{mM} \mathrm{KCl}$

$137 \mathrm{mM} \mathrm{NaCl}$

$1.5 \mathrm{mM} \mathrm{KH}_{2} \mathrm{PO}_{4}$

$8.1 \mathrm{mM} \mathrm{Na}_{2} \mathrm{HPO}_{4}$

pH 7.4 


\subsubsection{Preparation of Silicon Substrates used for Solid Supported Lipid Layers}

In this work solid supported bilayers were prepared on silicon wafers. Silicon wafers were purchased at Silicon Materials, Inc., Glenshaw, PA, USA coated with either $5000 \mathrm{~nm}$ silicon dioxide (used for RIfS experiments, chapter 3.4.1) or $100 \mathrm{~nm}$ silicon dioxide (used for fluorescence microscopy). SUVs (chapter 3.3.1) were spread on these silicon wafers in all cases. To obtain bilayers hydrophilic functionalized silicon wafers were used.

\section{Experimental procedure}

Silicon wafers were cut into $1.9 \mathrm{~cm} \times 0.8 \mathrm{~cm}$ rectangles independently of the used method (RIfS and CLSM). To produce hydrophilic silicon wafers, wafers were cleaned in an Argon plasma and subsequently put into an aqueous ammonia hydrogen peroxide solution $\left(\mathrm{H}_{2} \mathrm{O}: \mathrm{H}_{2} \mathrm{O}_{2}(30 \%): \mathrm{NH}_{3}(25 \%)\right.$ 5:1:1 ( $\left.\left./ \mathrm{v} / \mathrm{v}\right)\right)$ for 20 min at $70{ }^{\circ} \mathrm{C}$ using a Teflon holder. Functionalized wafers were at least cleaned with ultrapure water and stored in ultrapure water for a maximum of 12 hours. Wafers were placed into desired measuring chambers and covered with spreading buffer (Table 3.4$)$. Then, SUVs $\left(0.8 \mathrm{mg} \cdot \mathrm{mL}^{-1}\right)$ were added to induce spreading on the surface.

$\begin{array}{lll}\text { parameters for Ar-plasma treatment } & \text { process pressure: } & 0.2 \mathrm{mbar} \\ & \text { gas feed: } & 2 \mathrm{~min} \\ & \text { plasma duration: } & 30 \mathrm{~s} \\ & \text { power: } & 60 \%\end{array}$

\subsubsection{Preparation of Giant Unilamellar Vesicles}

Vesicles with a diameter above $1 \mu \mathrm{m}$ are called giant unilamellar vesicles (GUVs).

Angelova et al. first introduced a technique called liposome electroformation. ${ }^{[12]}$ The basic principle relies on rehydration of a lipid film under stabilization of a sinusoidal alternating current (AC) voltage. Therefore, some parameters must been set: Too high voltages and frequencies of the sinus wave can lead to vesicle rupturing. Also sugar solutions are widely used as an additional stabilization factor. ${ }^{[113]}$ The restriction to low salt conditions of the used buffer solution $(\leq 10 \mathrm{mM} \mathrm{NaCl})$ is the most significant disadvantage of this method.[114] 


\section{Experimental procedure}

Lipids diluted in chloroform and methanol $\left(c=0.2 \mathrm{mg} \cdot \mathrm{ml}^{-1}\right)$ were dispersed on two indium tin oxide layers (ITO slides) and dried under vacuum for at least 1 hour. Teflon spacer and a silicon ring were placed between the ITO slides to form an electroformation chamber (Figure 3.2). Self-adhesive copper strips on the spacers were used as electrodes. The chamber was filled with $1 \mathrm{M}$ sucrose solution $(V=1.7 \mathrm{~mL})$ and an AC voltage of $3 \mathrm{~V}$ (peak-to-peak) and a frequency of $5 \mathrm{~Hz}$ was applied. After 3 hours within the electric field the GUV suspension was collected. After every electroformation the ITO slides were cleaned with ethanol and water. Afterwards the slides were heated up to $150{ }^{\circ} \mathrm{C}$ to remove remaining lipid material. This mild annealing procedure is known to re-establish the original surface topography of the ITO slides.[115]

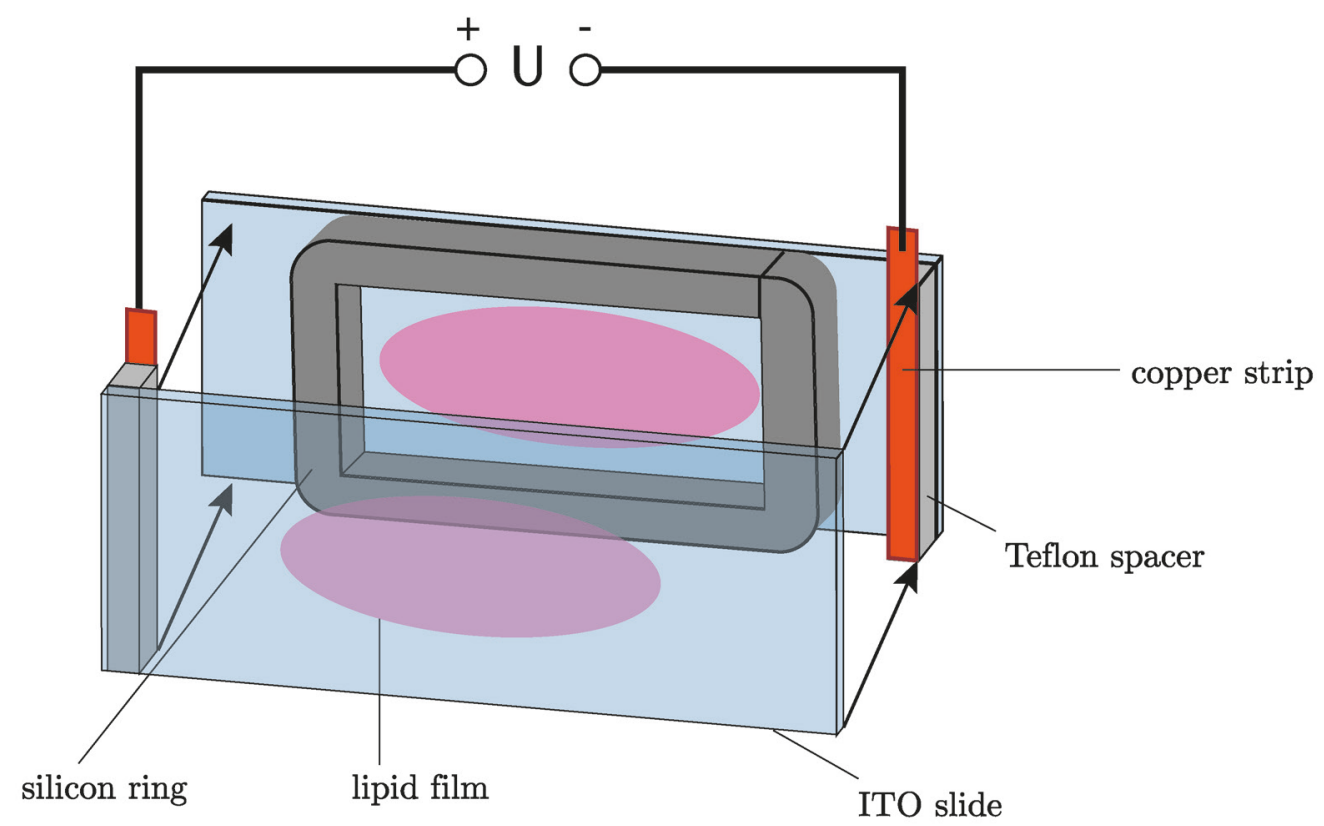

Figure 3.2: Schematic illustration of a GUV electroformation chamber. Lipid films were dried on ITO slides and a chamber was set up with a silicon ring. Both self-adhesive copper stripes, each on the Teflon spacers, were placed on the opposite ITO and connected to a voltage source.

\subsubsection{Preparation of Pore Spanning Lipid Bilayers}

The preparation of pore spanning lipid bilayers (PSLBs) is a more step procedure and schematically illustrated in Figure 3.3 A. The main steps are the functionalization and the subsequent spreading of GUVs. Briefly, the functionalization separates into evaporation and a self-assembled monolayer (SAM) of a thiol. The underlying $\mathrm{Si}_{3} \mathrm{~N}_{4}$ substrates are $5 \times 5 \mathrm{~mm}$ large and about $1 \mathrm{~mm}$ high. The pores of either wise $1.2 \mu \mathrm{m}$ or $5.0 \mu \mathrm{m}$ in diameter show a hexagonal array and are surrounded by one larger rim of 
$0.5 \mathrm{~mm}$. Substrates were purchased by Aquamarijn $(\varnothing=1.2 \mu \mathrm{m}$ and $5.0 \mu \mathrm{m}$, Zutphen, Netherlands) and fluXXion $(\varnothing=1.2 \mu \mathrm{m}$, Eindhoven, Netherlands). Scanning electron micrographs of a blank and an already evaporated substrate is shown in Figure 3.3 $\mathrm{B} / \mathrm{C}$.

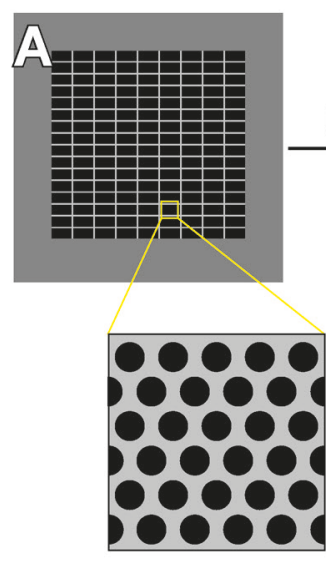

I: functionalization

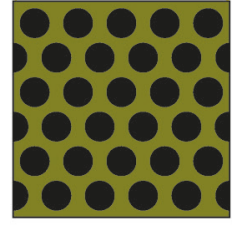

II: GUV spreading
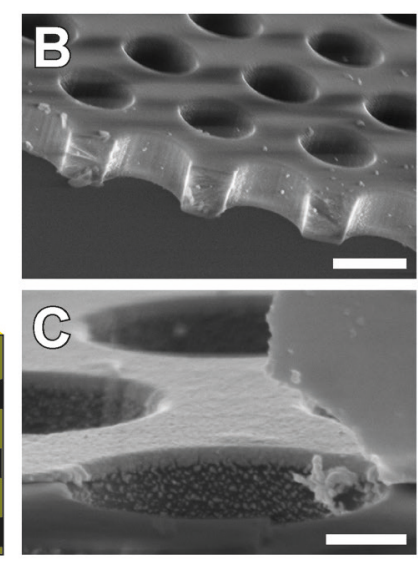

Figure 3.3: Schematic illustration of PSLB preparation and scanning electron micrographs of a fluXXion $\mathbf{S i}_{3} \mathrm{~N}_{4}$ substrate. A: Illustration of PSLB preparation. First, the $\mathrm{Si}_{3} \mathrm{~N}_{4}$ substrate must be functionalized by evaporation of titanium and gold and subsequent assembly of a thiol SAM (I). On this surface GUVs can be spread and with overstretching a pore a PSLB is formed. One GUV patch produces many PSLBs close to each other (last magnification). B: Scanning electron microscopy image of a blank fluXXion substrate with a pore diameter of $1.2 \mu \mathrm{m}$. C: An already evaporated substrate. ${ }^{[16]}$ The gold layer with a height of around $35 \mathrm{~nm}$ is clearly visible. Scale bars: A: $1 \mu \mathrm{m}, \mathrm{B}: 300 \mathrm{~nm}$.

\section{Functionalization of Porous Substrates}

Prior to functionalization substrates were cleaned by argon plasma under the same conditions as silicon wafer described in chapter 3.3.2. The clean substrate was directly coated with a thin layer of titan dioxide with a sputter coater (Cressington, Dortmund, Germany). Cathode sputtering was carried out in an argon plasma at 0.05 bar at a current strength of $40 \mathrm{~mA}$ for 30 seconds. Exposing the titan coating to ambient air led to oxidation resulting in a titan dioxide layer which served as adhesion promoter for subsequent gold vaporization.

Vaporization was implemented in a high vacuum of $10^{-6}$ mbar utilizing a turbomolecular pump (vaporization plant Bal-Tec MED 020 by Leica Microsystems GmbH, Wetzlar, Germany). The advantage of vaporization over sputter coating is faster and more homogeneous coating. ${ }^{[117]}$ Gold was provided in a tungsten boat which was heated up by increasing the current strength to $I \approx 60$ A. A layer of $35 \mathrm{~nm}$ gold was evaporated onto the titan dioxide coated silicon nitrite substrates with a deposition rate between 0.2 and $0.3 \mathrm{~nm} \cdot \mathrm{s}^{-1}$. Functionalization was completed by a self-assembly of a $\beta$-mercaptoethanol monolayer utilizing to a strong thiolate-gold bond 
$\left(160-210 \mathrm{~kJ} \cdot \mathrm{mol}^{-1}\right)$ via chemisorption. ${ }^{[118]}$ For that, the substrate was incubated in a $10 \mathrm{mM} \beta$-mercaptoethanol solution in isopropanol overnight, resulting in a hydrophilic surface due to the SAM.

\section{Spreading of GUVs on Functionalized Substrates}

Substrates were placed either wise in a fixing Teflon chamber or glued to the bottom of a petri dish. Subsequently, GUVs obtained from electroformation as described in chapter 3.3.3, were spread on the hydrophilic surface of functionalized substrates using a sedimentation technique as illustrated in Figure 3.4. A $5 \mathrm{~mL}$ Eppendorf tip was widened at the tip vent by cutting the end and hence converted to a buffer column filled with PBS buffer. $100 \mu \mathrm{L}$ of GUV suspension were given into the column separating GUVs from remaining lipid material. After one minute of GUVs passing through the column, the buffer column was removed, and GUVs could spread over 30 minutes. Afterwards, the substrate was rinsed $(10 \times 1 \mathrm{~mL})$ with PBS to remove remaining vesicles and incubated with bovine serum albumin (BSA, $50 \mu \mathrm{M}$ ) to passivate the remaining uncovered substrate rims for at least 30 minutes.

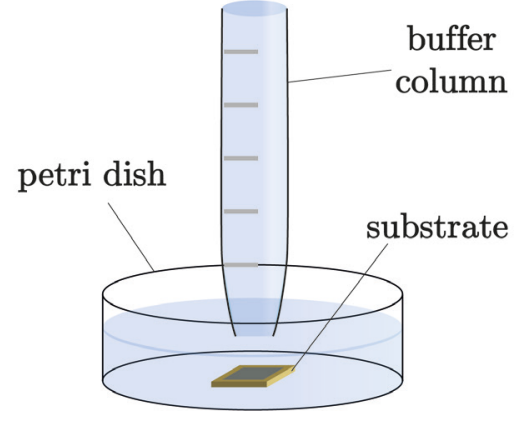

Figure 3.4: Schematic illustration of sedimentation technique. An Eppendorf tip is converted to a buffer column where GUVs were passing through to separate lipid material from vesicles.

\subsubsection{Polymerization of Actin-Networks}

F-actin networks were polymerized before attachment and were therefore called pre-polymerized F-actin networks. $1 \mathrm{mg}$ of either muscle (rabbit skeletal muscle, $>99 \%$, $\alpha$-actin) or non-muscle (human platelet, $>99 \%, 85 \% \beta$-actin, $15 \% \gamma$-actin) G-actin monomers (Cytoskeleton, Denver, CO, USA) were dissolved in ultrapure $\mathrm{H}_{2} \mathrm{O}$ and aliquoted into $10 \mu \mathrm{L}$ units at a concentration of $10 \mathrm{mg} \cdot \mathrm{mL}^{-1}$ and stored at $-80{ }^{\circ} \mathrm{C}$. Before use, G-actin was transferred in $238 \mu \mathrm{L}$ G-buffer to reach a concentration of $0.4 \mathrm{mg} \cdot \mathrm{mL}^{-1}$ and DTT $(0.5 \mathrm{mM})$ as well as ATP $(0.2 \mathrm{mM})$ were added to the solution. This was stored on ice for one hour to avoid the presence of oligomers. Subsequent centrifugation (17 $000 \mathrm{~g}, 20 \mathrm{~min}, 4^{\circ} \mathrm{C}$ ) and transfer into a new Eppendorf cup ensured removal of oligomers. Before inducing the polymerization, tracer particles or actin binding proteins (ABPs, cf. appendix 7.9) were added if desired. While ABPs were added in a distinct concentration or ABP to G-actin ratio, tracer particles were added in a volume of $1 \mu \mathrm{L}$ of a stock solution containing $0.8 \%$ solids and vortexed after addition. Stock solutions 
were prepared by five-time centrifugation (17 $000 \mathrm{~g}, 5 \mathrm{~min}, \mathrm{RT})$ and subsequent resuspension in ultrapure $\mathrm{H}_{2} \mathrm{O}$ of the purchased particle solution (Table 3.5).

Table 3.5: Characteristics and origin of used tracer particles.

\begin{tabular}{c|c|c|c|c} 
material & $\operatorname{size}(\varnothing)$ & $\lambda_{\mathrm{ex}}$ & $\boldsymbol{\lambda}_{\mathrm{em}}$ & manufacturer \\
\hline $\begin{array}{c}\text { polystyrene } \\
\text { carboxylate-modified }\end{array}$ & $\begin{array}{c}2 \mu \mathrm{m} \\
(1.9-2.1 \mu \mathrm{m})\end{array}$ & $575 \mathrm{~nm}$ & $610 \mathrm{~nm}$ & $\begin{array}{c}\text { Sigma-Aldrich, } \\
\text { St. Louis, MO, USA } \\
\text { silica particles } \\
\text { plain }\end{array}$ \\
$\begin{array}{c}2.01 \mu \mathrm{m} \\
(1.8-2.2 \mu \mathrm{m})\end{array}$ & non-fluorescent & $\begin{array}{c}\text { Bangs Labs, IN, } \\
\text { USA }\end{array}$
\end{tabular}

With or without additional agent polymerization was induced by adding $10 \%$ of the total volume polymerization solution (F1-solution). After $30 \mathrm{~min}$ of polymerization time Alexa Fluor ${ }^{\circledR}$ 488-phalloidin (Invitrogen, Carlsbad, CA, USA) was added in a $1.5 \%$ ratio, related to G-actin monomers, to label and stabilize the filamentous networks and avoid depolymerization (chapter 1.3). Pre-polymerized networks obtained in a concentration of $c=8 \mu \mathrm{M}$ were stored at $4{ }^{\circ} \mathrm{C}$ overnight.

To evaluate the quality of pre-polymerized F-actin networks the polymerized network gel was filled into microfluidic chamber or channel. These chambers were prepared by sticking double-sided adhesive tape (Tesa SE, Norderstedt, Germany) onto a microscope slide (Thermo Fisher Scientific, Waltham, MA, USA) and removing longitudinal rectangles with a scalpel as shown in Figure 3.5. After filling the F-actin gel inside the rectangle, the microfluidic chamber was closed with a coverslip. In case of microfluidic channels, F-actin was filled inside after attaching the coverslip by capillary forces. Channels were closed afterwards with Parafilm ${ }^{\circledR}$.

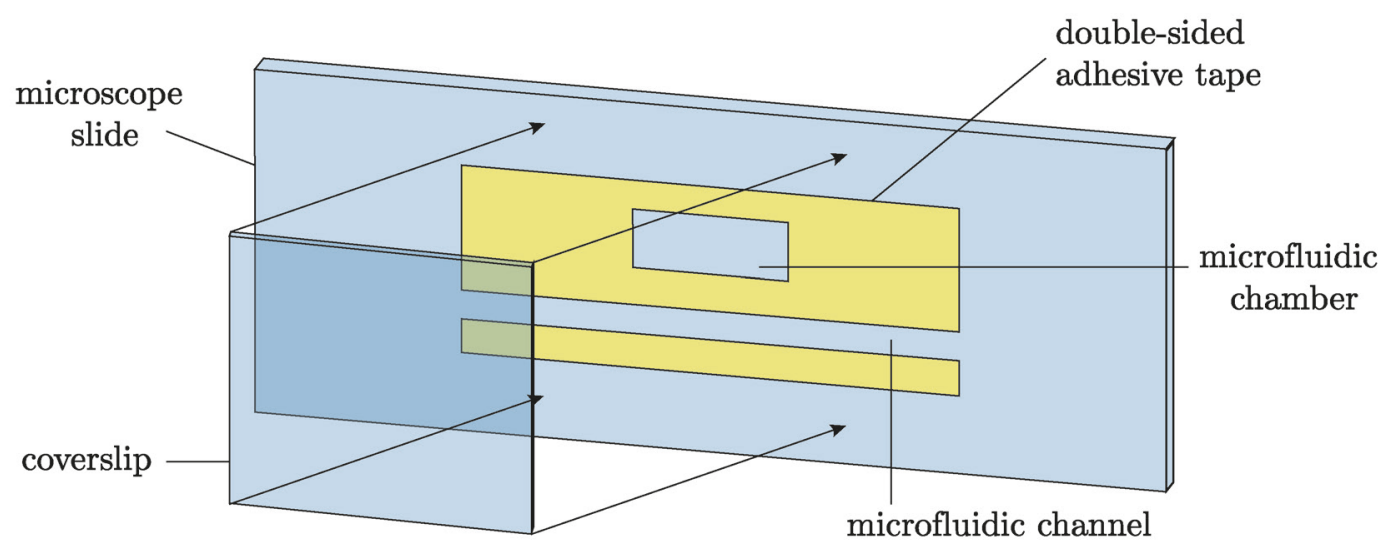

Figure 3.5: Schematic illustration of microfluidics chamber and channel construction. A double-sided adhesive tape is glued onto a microscope slide. Using a scalpel, rectangles in different dimension are cut out of the tape. After pipetting the F-actin gel into these rectangles the chamber is closed with a cover slip. The cut microfluidic channel was filled after cover slip mounting and closed with Parafilm ${ }^{\circledR}$. 
Further on, networks pre-polymerized as described above were also attached to poly-D-lysin surfaces for AFM analysis. Therefore, glass bottom dishes (MatTek Corp., Ashland, USA) were incubated overnight with poly-D-lysin $\left(c=0.1 \mathrm{mg} \cdot \mathrm{mL}^{-1}\right)$. After rinsing with F-buffer $10 \mu \mathrm{L}$ F-actin, polymerized as described above, was pipetted onto the poly-D-lysin incubated glass bottom of the dish. After at least 3 hours of incubation time the specimens were rinsed with F-buffer and used for AFM experiments.

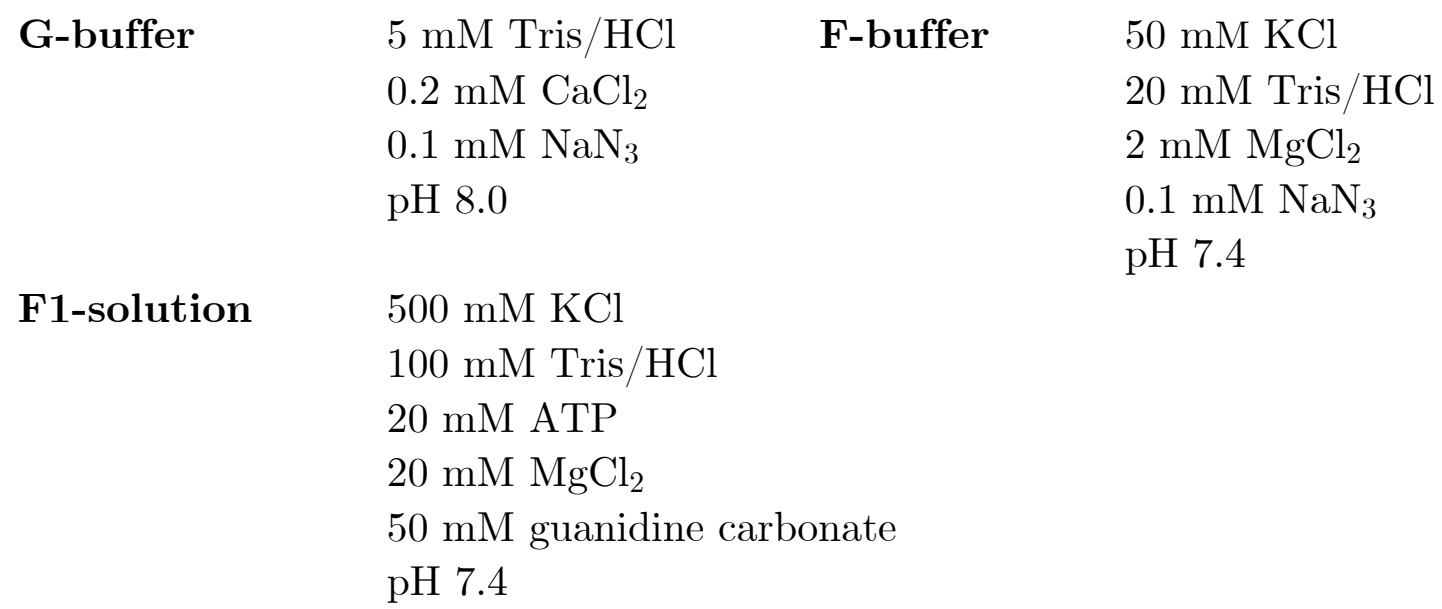

\subsubsection{Preparation of Minimal Actin Cortices}

Hydrophilic silicon substrates functionalized as described in chapter 3.3.2, were placed in a Teflon chamber and spreading buffer solution was added. Immediately afterwards the substrate was incubated with SUVs $\left(m=0.2 \mathrm{mg}, c=0.8 \mathrm{mg} \cdot \mathrm{mL}^{-1}\right)$ for 1 hour. While SUVs without PtdIns[4,5] $\mathrm{P}_{2}$ were spread in PBS buffer, SUVs with PtdIns $[4,5] \mathrm{P}_{2}$ were spread in Na-citrate buffer to ensure homogeneously distributed receptor lipids.[119] SUVs were prepared with desired lipid composition as described in chapter 3.3.1. Addition of either wise $0.4 \mathrm{~mol} \%$ TxR-DHPE or $1 \mathrm{~mol} \%$ Atto 390-DOPE was necessary to characterize lipid bilayers by means of fluorescence microscopy. After spreading of the vesicles excess lipid material was rinsed away with spreading buffer followed by a buffer exchange to protein buffer (E1 buffer) in case of using ezrin T567D (isolated and purified as described previously). Using neutravidin (Thermo Fisher Scientific, Waltham, MA, USA) this step is not required due to further proceed in PBS buffer. Subsequently, protein was incubated for binding $(c \approx 1 \mu \mathrm{M})$ at $4{ }^{\circ} \mathrm{C}$ overnight. The specimens were conditioned to room temperature by waiting for 1 hour without adding temperature before repeated rinsing with E1 buffer was used to remove unbound protein. Afterwards buffer solution was changed to F-buffer to optimize the conditions for F-actin binding, independently from the binding system. F-actin networks obtained under the conditions described in chapter 3.3.5 were than incubated for 4.5 hours to form minimal actin cortices on protein-decorated lipid layers. A last rinsing with F-buffer was implemented to remove unbound filaments. 


\subsection{Bio-sensing Methods}

\subsubsection{Reflectometric Interference Spectroscopy (RIfS)}

Reflectometric Interference Spectroscopy (RIfS) is a technique based on refraction of light at thin films. This method of measurement was developed by experiments of Langmuir and Schäfer who applicated a layer thicknesses detecting technique to protein monolayers. ${ }^{[120-122]}$ Major advantages of this technique are the high sensitivity and that it is a non-invasive method which does not need any fluorescence markers or chemical change of the protein for detection. This spectroscopic method relies upon the refraction of light at an interface of two media with different refraction indices $\left(n_{\mathrm{i}}\right)$. In this context Snell's law (equation 3.1) describes the relationship between the angles of incidence $(\theta)$ and refraction $(\phi)$ of an irradiated light beam: ${ }^{[123]}$

$$
n_{1} \cdot \sin (\theta)=n_{2} \cdot \sin (\phi)
$$

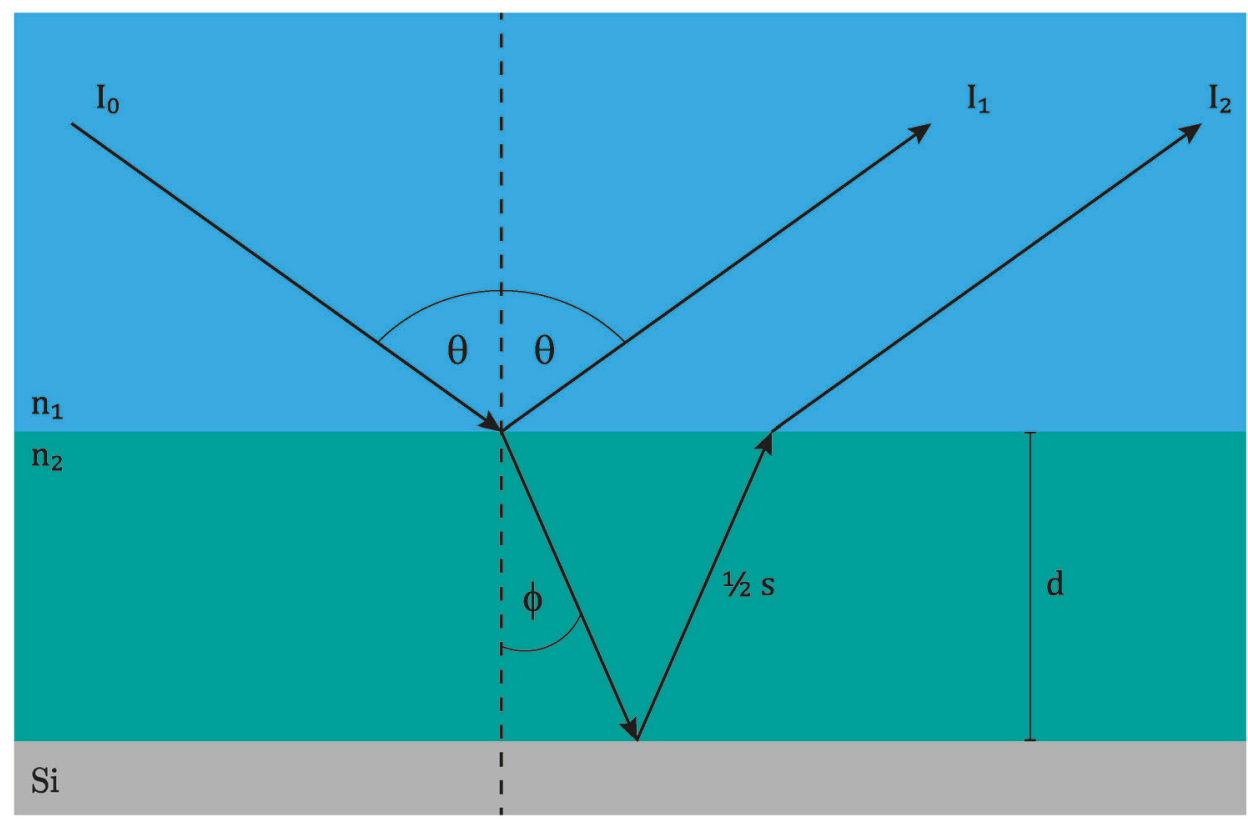

Figure 3.6: Illustration of the optical path in a RIfS setup utilizing Snell's law. Light $\left(I_{0}\right)$ traveling through the aqueous medium $\left(n_{1}\right)$ confronts the interface where it is partwise reflected $\left(I_{1}\right)$ and refracted $\left(I_{2}\right)$ according to Snell's law. Refracted light travels an additional path length $(s)$ which depends on the thickness $(d)$ of the second medium $\left(n_{2}\right)$. At the silicon interface the partial beam is reflected completely due to the high refractive index $\left(n=3.94, \lambda=600 \mathrm{~nm}, 20{ }^{\circ} \mathrm{C}\right) .{ }^{[124]}$

In the setup used in this work the first permeated medium is the aqueous buffer solution $\left(n_{1}=1.33, \lambda=600 \mathrm{~nm}, 20^{\circ} \mathrm{C}\right)^{[125]}$ while the second permeated medium is the silicon dioxide layer inclusively the functionalization of the surface $\left(n_{2}=1.55\right.$, average 
refractive index of $340 \mathrm{~nm} \leq \lambda \leq 991 \mathrm{~nm}$ at $\left.20{ }^{\circ} \mathrm{C}\right)^{[126]}$ as shown in Figure 3.6. Light $\left(I_{0}\right)$ traveling through the aqueous medium faces the interface where it is partially refracted $\left(I_{2}\right)$ and partially reflected $\left(I_{1}\right)$. According to equation 3.1 the refracted light is tilted to the vertical with the refraction angle $\phi$. At the interface to the silicon interface $\left(n_{3}=3.94, \lambda=600 \mathrm{~nm}, 20{ }^{\circ} \mathrm{C}\right)^{[124]}$ the refracted is reflected completely. Facing the interface, a second time, the refracted partial beam is tilted out of the vertical (according to equation 3.1) and travels parallel to the reflected light $\left(I_{1}\right)$ with reduced intensity compared to the initial light intensity $\left(I_{0}\right)$. The additional path length $(s)$ of the refracted light depending on the thickness of the second medium $(d)$ can be described with equation 3.2 :

$$
\cos (\phi)=\frac{2 d}{s} \quad \Leftrightarrow \quad s=\frac{2 d}{\cos (\phi)}
$$

Respecting the refractive index of the silicon dioxide layer $\left(n_{2}\right)$, the optical path length $\left(s_{\mathrm{OT}}\right)$ can be described as a function of the silicon dioxides optical thickness (OT, equation 3.3). The refractive indices of silicon dioxide, lipid bilayers and protein layers are in the same range, ranging from 1.36 to $1.50 .{ }^{[127]}$ Therefore, with adsorbing a lipid bilayer and subsequently protein the thickness of the refracting layer is getting increased and the optical thickness changes. The $O T$ is defined as the product of physical thickness $(d)$ and refractive index $(n)$ as shown in equation 3.4:

$$
\begin{gathered}
s_{\mathrm{OT}}=\frac{2 d \cdot n_{2}}{\cos (\phi)}=\frac{2 O T}{\cos (\phi)} \\
O T_{n_{2}}=d \cdot n_{2}
\end{gathered}
$$

In the available RIfS setup (Figure 3.7) the optical light source is mounted orthogonally to the specimen to prevent diffraction of light. Therefore, equation 3.3 simplifies in the following way, respecting $\theta=\phi=0$ :

$$
S_{O T}=20 T
$$

The difference in path lengths between $I_{1}$ and $I_{2}$ results in phase shift $(\Delta \varphi)$ leading to a distinct interference pattern. A constructive interference occurs if the additional path length $s_{\mathrm{OT}}$ is a multiple of the wavelength $\lambda$ : 


$$
m \cdot \lambda_{\max }=2 \text { OT }[m \in \mathbb{N}]
$$

On the other hand, destructive interference occurs if both wavelengths have an internal shift of half of the wavelength:

$$
\left(m+\frac{1}{2}\right) \cdot \lambda_{\min }=2 \text { OT }[m \in \mathbb{N}]
$$

Therefore, the phase shift can be set in a relationship to the optical thickness respecting the case of a perpendicular incidence of light $(\theta=\phi=0)$ :

$$
\Delta \varphi(\lambda)=\frac{2 \pi}{\lambda} \cdot O T \cdot \cos (\phi) \Rightarrow \Delta \varphi(\lambda)=\frac{2 \pi}{\lambda} \cdot O T
$$

Any kind of molecules, in context of this work lipids and proteins, which are adsorbed to the interface thickens the physical layer thickness and therefore the interference pattern. With equation 3.8 in hand the change in optical thickness can be tracked by the phase shift.

First, reference spectra with a silver evaporated glass substrate ( $I_{\mathrm{r}}$, light reference, reflectivity $>98.5 \%)$ and in darkness $\left(I_{\mathrm{d}}\right)$ were recorded with the spectrometer (Ocean Optics Inc., Dunedin, FL, USA). The program Spectra Suite recorded a reflectivity spectrum $R$ with $R$ deriving from measured intensity $\left(I_{\mathrm{m}}\right)$ according to equation 3.9.[128] In order to calculate the reflectance and transmittance describing coefficients are needed. For this purpose, Fresnel coefficients, still assuming a perpendicular angle of incidence, are defined by equation 3.10.[129] Concerning the geometry of the setup used in this work, the resulting reflectivity is described with the Fresnel coefficient $r_{123}$ (equation 3.11).[130]

$$
\begin{gathered}
R=\frac{I_{m}-I_{d}}{I_{r}-I_{d}} \\
r_{x y}=\frac{n_{x}-n_{y}}{n_{x}+n_{y}} \\
R(\varphi)=r_{123}^{2}=\frac{r_{12}^{2}+r_{23}^{2}+2 \pi r_{12} r_{23} \cdot \cos (2 \varphi)}{1+r_{12}^{2}+r_{23}^{2}+2 \pi r_{12} r_{23} \cdot \cos (2 \varphi)}
\end{gathered}
$$


A combination of equation 3.11 and 3.8 which therefore means putting the phase shift in context to the reflectivity leads to the dependency of the distinct interference pattern and the optical thickness of the layer:

$$
R(\lambda, O T)=r_{123}^{2}=\frac{r_{12}^{2}+r_{23}^{2}+2 \pi r_{12} r_{23} \cdot \cos \left(\frac{4 \pi}{\lambda} O T\right)}{1+r_{12}^{2}+r_{23}^{2}+2 \pi r_{12} r_{23} \cdot \cos \left(\frac{4 \pi}{\lambda} O T\right)}
$$

To track the change in optical thickness in a time resolved manner a MATLAB script with a graphical user interface using equation 3.12 written by Dr. Ingo P. Mey (Georg-August-Universität Göttingen) was utilized.

\section{RIfS measurement setup}

The typical measuring chamber of a RIfS measurement is schematically illustrated in Figure 3.7. The bottom part is an alumina base plate with an integrated slot of $4 \mathrm{~cm}^{2}$ where the functionalized silicon substrate was placed into. A seal ring determining the size of the chamber was pressed onto the alumina base plate with a cap of acrylic glass. In- and outflow were assembled to the acrylic glass cap as shown in Figure $3.7 \mathrm{~A}$. The acrylic glass cap is mounted with screws onto the alumina base plate. The heart of the setup is the optical fiber which was mounted on top on the acrylic glass cap in orthogonal direction to keep the restrictions of $\theta=\phi=0$. The optical fiber consists of six emitting fibers arranged in a circle around one light-collection fiber. Light is deriving from a tungsten halogen lamp $(\lambda=350-950 \mathrm{~nm})$ and collected light was sent to a $\mathrm{UV} / \mathrm{V}$ is silicon CCD detector with 2048 pixels.
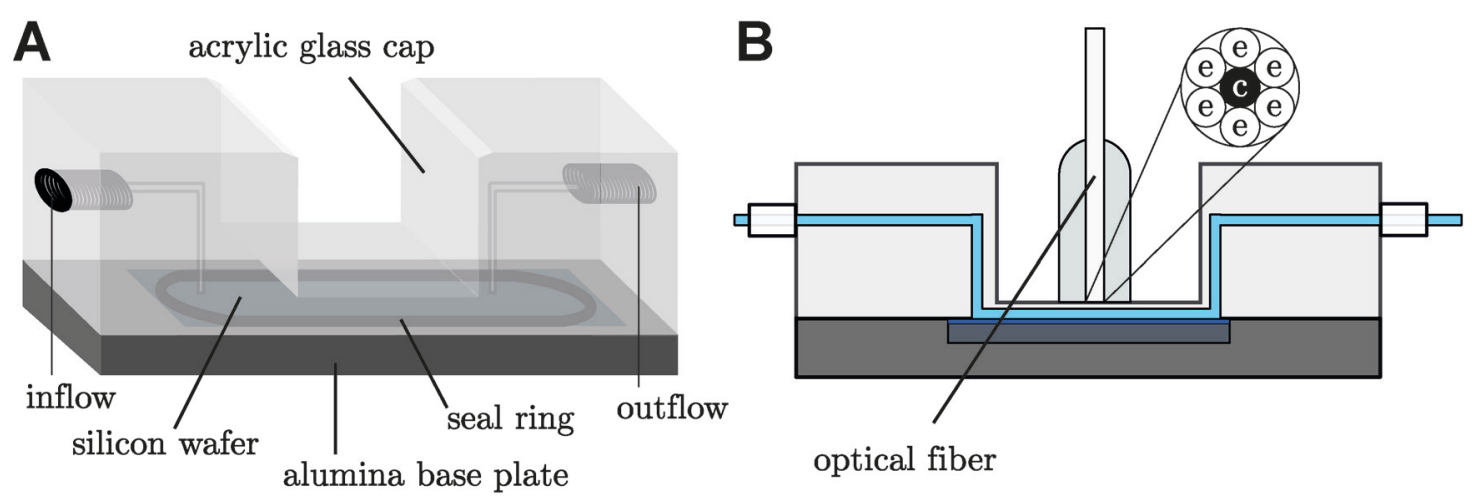

Figure 3.7: Schematic illustration of a RIfS measuring chamber. A: A plastic illustration shows the total setup of a RIfS measuring chamber. An acrylic glass cap with holes for in- and outflow is screwed onto the alumina base plate with inlaying silicon wafer. A seal ring completes the flow-through-chamber. B: The optical fiber is placed into the indentation in the acrylic glass cap. The fiber consists of six emitting (e) and one collecting (c) fiber. Graphics were kindly provided by Jonas Schäfer. 
Flow velocity and direction of the tube system was regulated with a peristaltic pump at $0.7 \mathrm{~mL} \cdot \mathrm{min}^{-1}$.

\section{RIfS measurement implementation}

After the data point recording was started utilizing Spectra Suite the system was equilibrated with the respective spreading buffer. Rinsing with buffer always occurred in an open system, whereas every addition of protein and lipids was performed in a closed configuration. Addition of SUVs $(m=0.2 \mathrm{mg})$, prepared as described in chapter 3.3.1, was tracked till a baseline set in and then the system was rinsed with spreading buffer again. After the change to protein buffer (E1 buffer in case of Ezrin T567D) protein $\left(c_{\text {ezrin }}=0.8-1 \mu \mathrm{M}\right)$ was added. Concentration was chosen to provide enough protein to saturate the whole functionalized surface. ${ }^{[131]}$ As soon as a plateau was reached again, the system was rinsed once more with protein buffer to obtain an information about irreversible or unbound protein. The flow system was cleaned by flushing the tube system with ultrapure water and measuring chambers were cleaned three times in an ultrasonic bath ( 1 x 15 min Mucasol ${ }^{\circledR}, 2$ x 15 min ultrapure water).

\subsubsection{UV/Vis Spectroscopy}

For determination of protein concentrations and to detect a degree of labeling (DOL) after protein labeling $\mathrm{UV} / \mathrm{V}$ is spectroscopy was used. The basic principle is the adsorption of light in the detectable range of ultraviolet (UV) and visible light (Vis). In case of proteins the $\pi \rightarrow \pi^{*}$ transition within amino acid residues with a conjugated $\pi$-electron system is responsible for the adsorption of light around a wavelength of $280 \mathrm{~nm}$. The residues responsible for this transition are phenylalanine, tryptophan and tyrosine. Therefore, the concentration of an aqueous protein solution can be determined via the adsorption at the wavelength $280 \mathrm{~nm}\left(A_{280}\right)$ using a protein specific extinction coefficient $\left(\varepsilon_{280}\right)$ with the law of Lambert-Beer (equation 3.13):

$$
A_{280}=\lg \frac{I_{0}}{I}=\varepsilon_{280} \cdot c \cdot d
$$

The quotient of light intensity before $\left(I_{0}\right)$ and after $(I)$ traveling through the cuvette with a specific thickness $(d)$ is needed to calculate the concentration of the protein $(c)$. The extinction coefficient for ezrin and ezrin T567D is $66935 \mathrm{M}^{-1} \cdot \mathrm{cm}^{-1}$. [132] $\mathrm{UV} / \mathrm{Vis}$ 
measurements were employed with the NanoDrop ${ }^{\mathrm{TM}}$ 2000c (Thermo Fisher Scientific, Waltham, MA, USA).

In case of labeled proteins, the adsorption of the respective fluorophore must be taken in account. Here, $A_{280}$ appears to be higher due to the additional adsorption of the fluorophore, thus a correction factor $\left(C F_{280}\right)$ must be respected. The correction factor is provided directly from the manufacturer. For Atto 488 used in this work it is $C F_{280}=0.09$. ${ }^{[133]}$ The protein concentration will be determined with equation 3.14 :

$$
c=\frac{\left(A_{280}-A_{\max , F}\right) \cdot C F_{280}}{\varepsilon_{280}}
$$

Furthermore, the already mentioned DOL is a well-suited parameter for the quality of the labeled protein. This value displays how many fluorophores are bound to the protein as it is defined as the quotient of protein concentration $\left(c_{\mathrm{P}}\right)$ to fluorophore concentration $\left(c_{\mathrm{F}}\right)$. The DOL is determined by equation 3.15 :

$$
D O L=\frac{c_{F}}{c_{P}}=\frac{A_{\max , F} / \varepsilon_{\max , F}}{A_{280, P} / \varepsilon_{280, P}}=\frac{A_{\max , F} \cdot \varepsilon_{280, P}}{\left(A_{280, P}-A_{\max , F} \cdot C F_{280}\right) \cdot \varepsilon_{\max , F}}
$$

$A_{\text {max }, \mathrm{F}}$ is the adsorption of the fluorophore at its adsorption maximum $\left(\lambda_{\max }\right)$ and $\varepsilon_{\max , \mathrm{F}}$ is the extinction coefficient at this wavelength. This coefficient is again given by the manufacturer.[133] 


\subsection{Biophysical Techniques}

\subsubsection{Atomic Force Microscopy}

Atomic force microscopy (AFM) was originally developed by Gerd Binning, Calvin Quate and Christoph Gerber in 1985. It was used to investigate topological structures of surfaces as this microscope showed a lateral resolution of $30 \AA$ and a vertical resolution less than $1 \AA$.[134] The typical setup of an atomic force microscope is shown in Figure 3.8 and consists of the cantilever with a sharp tip mounted to a piezoelectric actuator. A laser beam is positioned at the cantilever, which is reflected to a sensitive photo diode with four quadrants (A-D).

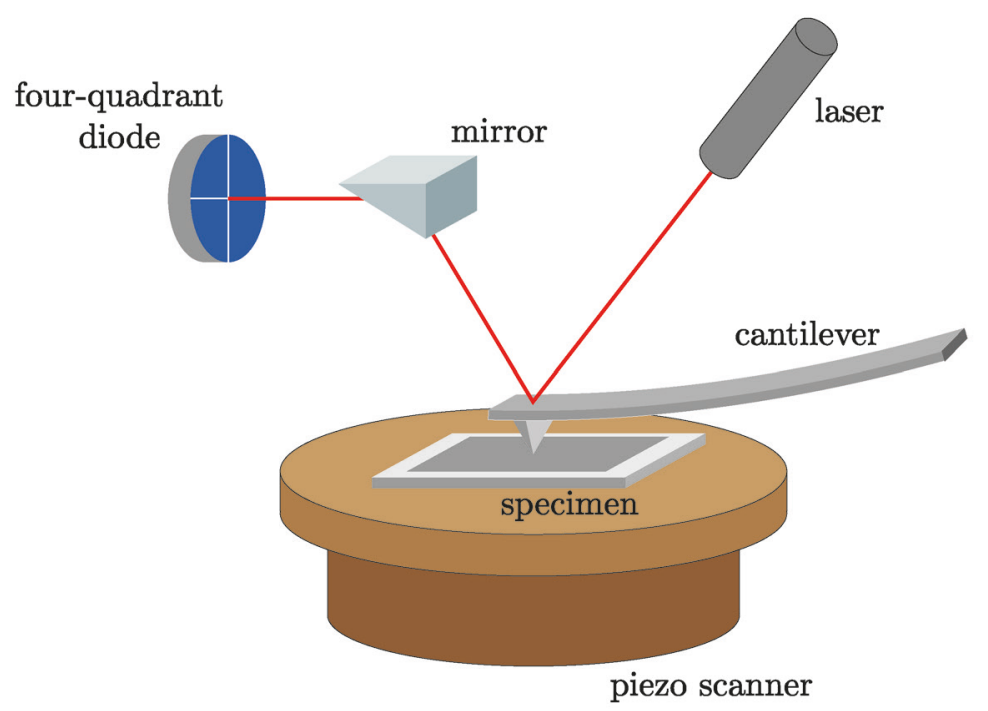

Figure 3.8: Schematic illustration of the basic AFM principle. A laser beam is positioned on the back of a cantilever which is scanning the specimen's surface. The reflected laser beam is deflected by a mirror to a four-quadrant diode which determines the deflection of the laser beam.

The basic principle of an AFM measurement is that the cantilever tip is scanning the specimen's surface with a specific feedback mechanism, which enables the z-piezo to keep the tip either at constant height or at constant force. During the scan the tip moves up and down depending on the character of the surface and the reflected laser beam is displaced on the photo sensitive diode with four quadrants. Therefore, the vertical deflection (in \%) can be set as a function of the intensity changes of each quadrant, as shown in equation 3.16. The horizontal deflection can be determined in an analogous way.

$$
A_{\text {vert. deflection }}=\frac{I(\mathrm{~A}+\mathrm{B})-I(\mathrm{C}+\mathrm{D})}{I(\mathrm{~A}+\mathrm{B})+I(\mathrm{C}+\mathrm{D})}
$$


This difference signal will be used as feedback to either keep the constant force or the constant height. In constant height mode the deflection force of the cantilever is recorded while in constant force mode the height deviation is monitored.[135]

In this work triangular MLCT cantilevers (Bruker AFM probes, Calle Tecate, CA, USA) with a tip length of 2.5-8.0 $\mu \mathrm{m}$ and a tip radius of 20-60 $\mathrm{nm}$ were used as shown in Figure 3.9. ${ }^{[136]}$ The cantilever back is covered with aluminum to ensure the reflection of the laser beam.

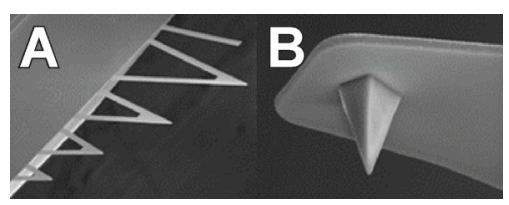

Figure 3.9: SEM images of MLCT cantilever (A) and cantilever tip (B).

\subsubsection{Indentation Experiments}

Topographical imaging is not the only way to use an AFM. Further development of the technique revealed a method to determine mechanical properties of surfaces by force-distance-curves. In this case, the cantilever gets in contact with the surface and is deviated with a specific force $F$. The dependence of the deviation $Z_{\text {c }}$ on the applied force is described by Hooke's law (equation 3.17):

$$
Z_{\mathrm{c}}=\frac{F}{k_{\mathrm{c}}}=\frac{4 \cdot F \cdot L_{\mathrm{c}}{ }^{3}}{E \cdot w_{\mathrm{c}} \cdot t_{\mathrm{c}}}
$$

The spring constant of the cantilever $k_{\mathrm{c}}$ is dependent on the length $L_{\mathrm{c}}$, width $w_{\mathrm{c}}$, thickness $t_{\mathrm{c}}$ and the modulus of elasticity $E$. Therefore, smaller spring constants can be used to determine smaller forces. Cantilever with very small spring constants can be affected easily by thermal fluctuations which lead to a worse signal-to-noise ratio. Nevertheless, Hooke's law is valid for frequencies below the resonance frequency and as presented in equation 3.17 only for a rectangular geometry. Therefore, a more effective method to determine the cantilever's spring constant was reported by Hutter et al.[137] They presumed the cantilever to behave like a harmonic oscillator and were able to determine the spring constant using a thermal noise spectrum. Additionally, the medium must be considered which leads to the method of Sader et al. described with equation 3.18.[138]

$$
k_{\mathrm{c}}=0.1906 \cdot \rho_{f} \cdot w_{\mathrm{c}}{ }^{2} \cdot L_{c} \cdot Q \cdot \Gamma_{\mathrm{i}}\left(\nu_{\mathrm{k}}\right) \cdot \omega_{0}^{2}
$$

Newly introduced are the parameters of $\rho_{f}$, the density of the immersed fluid, $Q$, the quality factor, $\Gamma_{\mathrm{i}}\left(v_{\mathrm{k}}\right)$, the imaginary part of the hydrodynamic function, and $\omega_{0}$ the resonance frequency of the cantilever obtained from thermal noise.

The calibrated dependence of Hooke's law is used to calculate the resulting force by deviation of the cantilever. To record a force-distance-curve (FDC) the cantilever is lowered using the $z$-piezo till the contact point with the surface is reached (Figure 3.10, non-contact regime, cyan line). Until now, no force acts on the cantilever and the laser beam on the photo diode is not displaced. Close to the contact point which is illustrated 
as $c p$ in the exemplary force distance curve shown in Figure 3.10 a snap on effect occurs due to the interaction with the surface. Then, the cantilever is lowered (contact regime), the detected signal of the photo diode increases linearly till the adjusted maximum force, the trigger point, is reached $(t p)$. The $z$-piezo is retracted (red line) showing the same behavior as the path towards the trigger point. If adhesive forces occur, a snap off effect is observable as illustrated in Figure 3.10. The deflection of the cantilever is connected to a voltage difference measured with the photo diode (Figure 3.10 A). To determine the accurate height displacement of the cantilever tip, the sensitivity, typically given in $\mathrm{nm} \cdot \mathrm{V}^{-1}$, must be quantified. For that, an FDC on a hard surface is recorded as the cantilever is deviated for the exact way which also the z-piezo is moved (Figure 3.10 B). Using Hooke's law, the deviation can be calculated to a force (Figure $3.10 \mathrm{C}) .[139]$
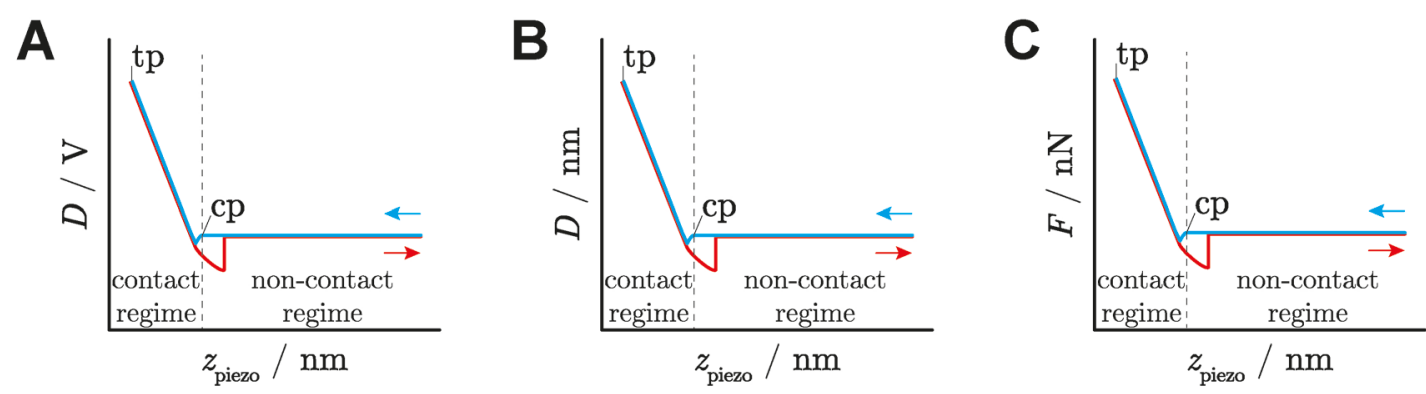

Figure 3.10: Schematic representation of a typical FDC. Trace (cyan) and retrace (red) are shown and recalculation of the feedback signal is displayed from A to B. A: The movement of the $z$-piezo ( $z_{\text {piezo }}$ in $\mathrm{nm}$ ) is obtained as voltage difference of the photo diode due to displacement in the four quadrants ( $D$ in V). B: Using the analyzed sensitivity of the cantilever a height deviation $(D$ in $\mathrm{nm}$ ) in dependence of the z-piezo movement is determined. C: Applying Hooke's law a force $(F$ in $\mathrm{nN})$ can be shown in dependence on the $z$-piezo movement. A forcedistance-curve is the result of the conversion.

The in the beginning mentioned method of topographical imaging of a surface using constant contact (contact mode) can lead to deformations of soft surfaces. Depending on the applied setpoint strong lateral forces act on the surface. To overcome these lateral forces the quantitative imaging mode (QITM mode) developed by $J P K$ instruments (Berlin, Germany) was used. The height information of the specimen is determined for each pixel via a single FDC. In contrast to typical force mapping experiments the FDCs are faster collected resulting in non-linear FDCs which makes the $\mathrm{QI}^{\mathrm{TM}}$ mode unsuitable for full elastic specimens. The interaction force can be controlled and minimized below $50 \mathrm{pN}$. ${ }^{[140]}$ The basic tip movement algorithm of the $\mathrm{QI}^{\mathrm{TM}}$ mode is illustrated in Figure 3.11. 


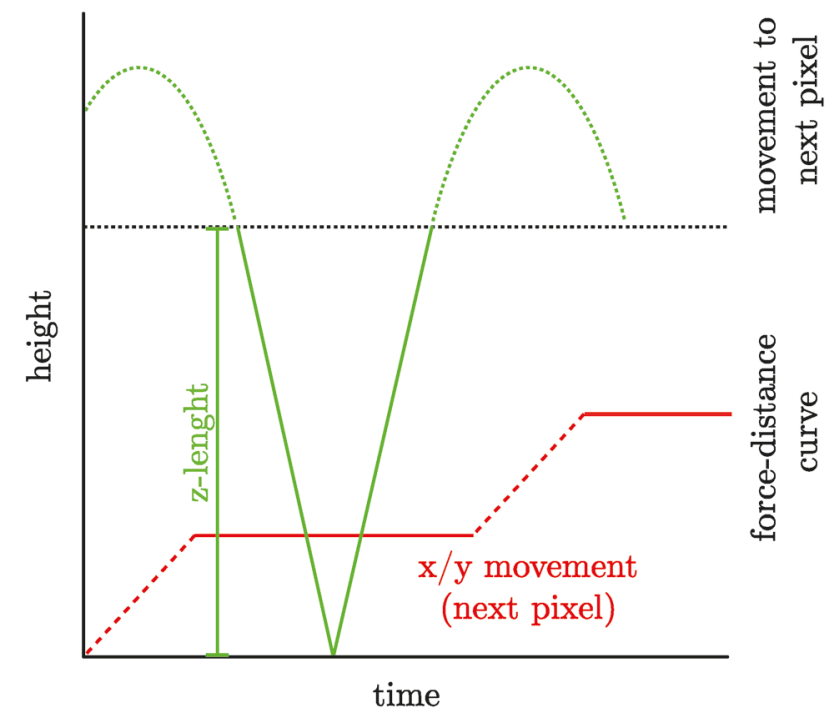

Figure 3.11: Illustration of tip movement using the $\mathrm{QI}^{\mathrm{TM}}$ mode. ${ }^{[140]}$ When the forcedistance curve is recorded (green solid line) no movement in $x$ - or $y$-direction (red line) occurs. Movement to the next pixel is only utilized when the tip is higher than the adjusted z-length.

While the FDC is recorded (Figure 3.11, green line) only a movement in $z$-direction performed while no movement in $x$-direction (as well as $y$-direction, Figure 3.11, red line) ensures no application of lateral forces. Height of the cantilever tip during no movement in $x-y$-direction is set by the $z$-length. Furthermore, the FDC is measured under constant velocity, which is necessary for accurate adhesion data and a dynamic baseline determination considers hydrodynamic effects. Thereby, the height information can be determined by trigger and contact points and the topographical image is calculated. ${ }^{[140,141]}$ 


\section{AFM measurement implementation}

A typical AFM measurement started with cantilever calibration after Sader et al. as described before. Sensitivity of the cantilever tip was determined by performing a single FDC using a setpoint of $1.5 \mathrm{~V}$ and a velocity of $1 \mu \mathrm{m} \cdot \mathrm{s}^{-1}$. Topographical QI image recording was done using a setpoint of $0.15 \mathrm{nN}$, a z-length of at least $100 \mathrm{~nm}$ and a pixel dwell time of $6 \mathrm{~ms}$. Positions for FDC curves were selected based on the QI image as illustrated in Figure 3.12. The approaching speed was constantly set to $1 \mu \mathrm{m} \cdot \mathrm{s}^{-1}$, z-length was at least $100 \mathrm{~nm}$ and the trigger point was set to $0.5 \mathrm{nN}$ if not mentioned separately.

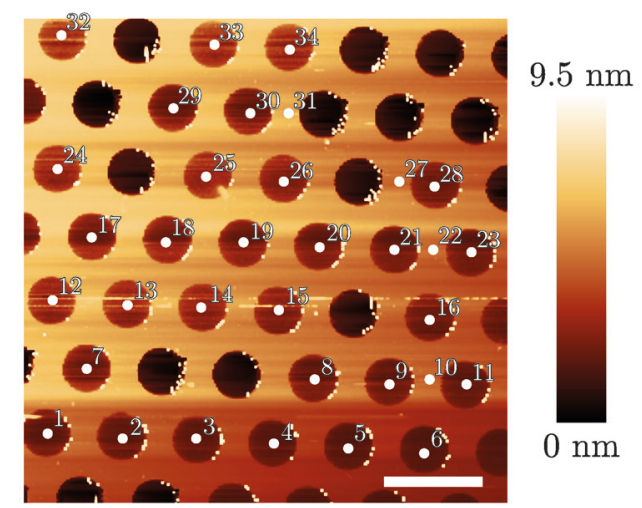

Figure 3.12: Principle of a measurement procedure using the $\mathrm{QI}^{\mathrm{TM}}$ mode. The atomic force micrograph was obtained using the QI mode. Afterwards, FDCs were recorded in the middle of a PSLB, which is illustrated by white marks. Occasionally, FDCs were recorded on the pore rim as observable on the right side $(10,22,27,31)$. Scale bar: $10 \mu \mathrm{m}$.

The contact point of FDCs were set to zero and evaluated using a MATLAB script by Dr. Ingo P. Mey (Georg-August-Universität Göttingen). The slope of the FDC is dependent on the measured surface and therefore evaluated as it displays the apparent spring constant $\left(k_{\mathrm{app}}\right)$ of the surface material. 


\section{Fluorescence Microscopy and AFM Co-Localization using the BioMat ${ }^{\text {TM }}$ Workstation}

A further tool to obtain more information about a single specimen is the use of a co-localization setup of AFM and fluorescence microscopy (cf. chapter 3.5.2). The BioMat $^{\mathrm{TM}}$ workstation, provided by JPK instruments, serves as shuttle station between the epifluorescence microscope and an AFM. For calibration, a calibration measuring chamber, identical in construction to the specimen's chamber, with an internal evaporated chromium adjustment cross was used (Figure 3.13). An AFM image was recorded, and the cantilever was calibrated directly to the intersection of the adjustment cross. Transferring this chamber, identical in position, to the fluorescence microscope, the adjustment cross can be recorded by means of fluorescence microscopy. Images of the intersection cross, recorded with both techniques, are shown in Figure 3.13 .

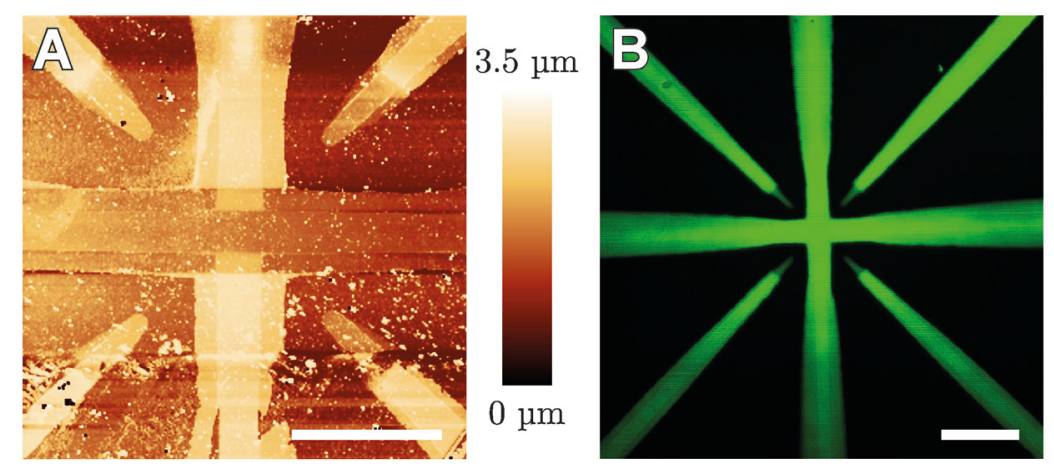

Figure 3.13: The adjustment cross recorded either by AFM or by fluorescence microscopy. A: Adjustment cross is set to measurement range of the AFM and the tip is directly calibrated to the cross intersection. B: The same spot is recorded by fluorescence microscopy and both measurement ranges are calibrated to each other for co-localization. The chromium cross can be detected due to reflection of light. Scale bars: A: $20 \mu \mathrm{m}, \mathrm{B}: 100 \mu \mathrm{m}$.

The cantilever tip is then successfully calibrated to specific position of the fluorescence microscope. Utilizing the BioMatTM shuttle station, the chamber with the adjustment cross can be exchange to the measuring chamber. To correlate a position, adjusting screws at the BioMat ${ }^{\mathrm{TM}}$ workstation allow a movement of the chamber, while the cantilever tip remains adjusted to the same position shown in the of the fluorescence microscope. 


\subsubsection{Confocal Laser Scanning Microscopy}

The technique of fluorescence microscopy is one of the most used techniques in the field of biochemistry, biology and physics. Specimen which carry a fluorescent dye can be visualized. The basic principle relies on the fact that a fluorophore can be excited by absorption of photons using light with a fluorophore specific wavelength. Deactivation occurs with the emission of light which is shifted to longer wavelengths in contrast to the excitation wavelength due to energy dissipation. This phenomenon is the so-called Stokes shift and is used to achieve higher contrast even at simultaneous excitation and emission. ${ }^{[142]}$ A further development of fluorescence microscopy is the confocal laser scanning microscopy (CLSM) which setup is shown in Figure 3.14.

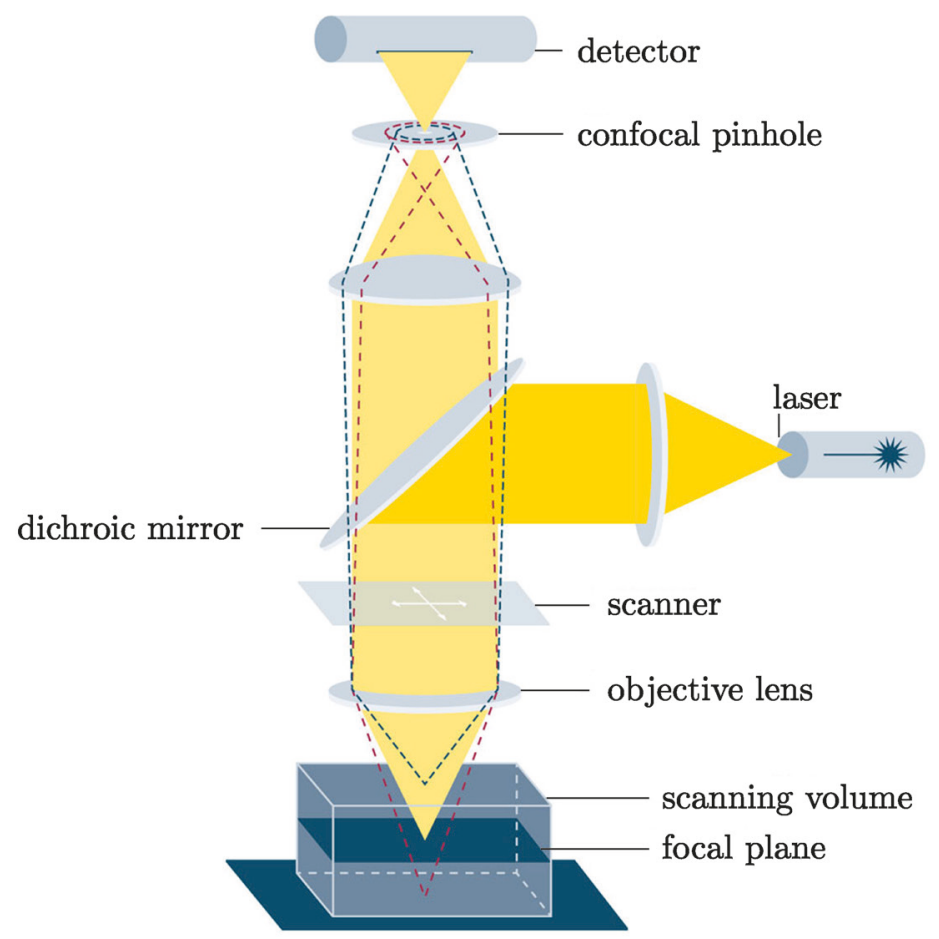

Figure 3.14: Schematic illustration of the principle of Confocal Laser Scanning Microscopy. ${ }^{[143]}$ A laser is used to illuminate the specimen's fluorophore by reflecting the laser onto the specimen with a dichroic mirror. The red-shifted fluorescence signal of the fluorophore passes through the dichroic mirror and is focused through the confocal pinhole until it reaches the detector. In-focus information (yellow) is focused exactly through the pinhole while out-of-focus information (red and blue dotted lines) get caught at the pinhole. A specific information of the focal plane is collected.

First, a monochromatic laser with desired wavelength is used to excite the specimen's fluorophore by focusing the light with an objective lens. A dichroic mirror, which reflected the light towards the specimen, lets the red shifted light passing through, so a further lens can focus the light through a confocal pinhole to the detector. Utilizing a confocal pinhole, only light out of the focal plane will be detected (yellow), as 
out-of-focus information (red and blue dotted lines) are excluded by the pinhole. Therefore, a systematic scan in $x$-y-direction will lead to a visualized information for a small volume which is illustrated as the focal plane. The pinhole diameter can be used to modify the detected volume. Infinitesimal small pinhole diameters would lead to a maximum resolution improvement of factor 1.4 in contrast to a wide-field microscope. ${ }^{[144]}$

The detectors used are typically conventional photomultiplier tubes (PMTs) or even more sensitive gallium arsenide phosphide (GaAsP)-PMTs. In order to obtain 3D information, many $x$-y-slices, each corresponding to one $z$-plane, can be recorded and combined into one stacked image. A spatial representation of the specimen can be achieved. These so-called z-stack images are used to determine height information out of the specimen.

Using CLSM or fluorescence microscopy in general, the most important factor is the achieved resolution of the setup. How a point like emitter is detected after the transmission through an objective with a high numerical aperture (NA) is described by the point-spread-function (PSF). The PSF is highly dependent on the used system and wavelength $(\lambda)$. As an objective is not able to focus light to infinitesimal small points interference occurs and a typical pattern of the PSF is detected (cf. Figure 3.15).
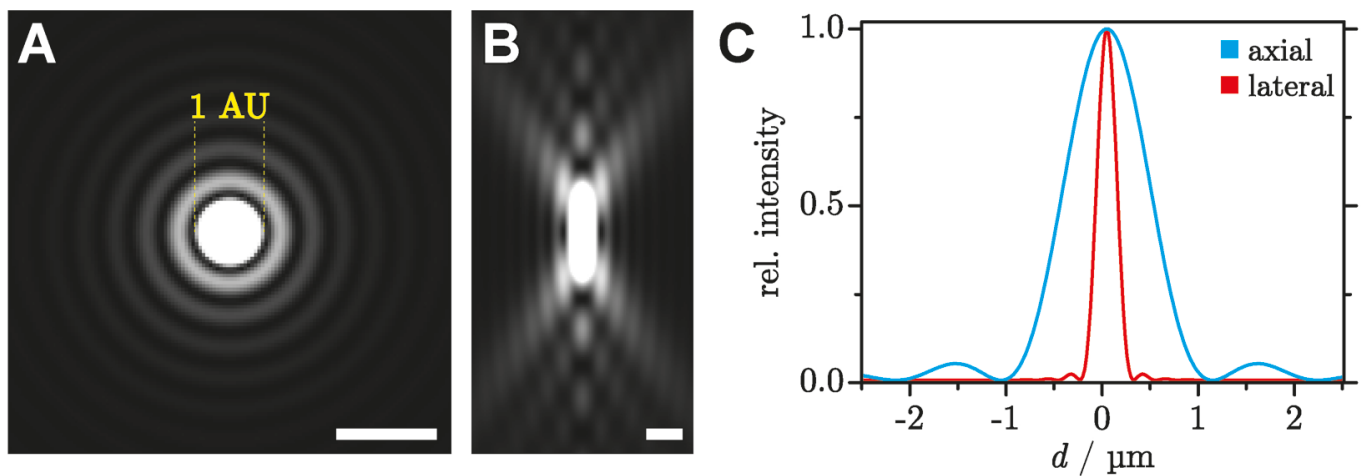

Figure 3.15: Representation of the point-spread-function using the ImageJ plugin PSF Generator. Parameters were set to: $\mathrm{NA}=1.0, \lambda=500 \mathrm{~nm}, n=1.33 .{ }^{[145]}$ A: PSF in $x^{-}$ $y$-plane. One airy unit is illustrated at the first interference minimum. B: PSF in $x$-z-plane. C: Normalized intensity profiles of the resulting calculated PSF. Scale bars: $500 \mathrm{~nm}$. Based on the idea of M. Gleisner. ${ }^{[146]}$

As Figure 3.15 reveals, the geometric pattern of the lateral and the axial profile differs which originates by different alternating series of interference patterns. It is clearly to see that the lateral resolution is better than the axial one. The lateral pattern of the PSF is a so-called airy disc and the diameter of the first intensity minimum is defined as airy unit (AU) as illustrated in Figure $3.15 \mathrm{~A}$. Resolution is now defined as the minimal distance, which is required to distinguish two PSF intensity profile (Figure 3.15 C). Regarding the Rayleigh criterion, the intensity of both objects has to drop to 
$73 \%$ of the maximum which allows a differentiation of both points. The width of the PSF highly depends on the wavelength, additionally to the mentioned NA. As the Rayleigh criterion is hard to measure, the most practical way to determine the resolution is to calculate the full width at half maximum (FWHM). The resolution in axial and lateral dimension of a CLSM using very small pinhole diameter is then given by equation 3.19 and 3.20 .

$$
\begin{gathered}
d_{\text {FWHM,lat }}=\frac{0.37 \cdot \bar{\lambda}}{\mathrm{NA}} \\
d_{\text {FWHM }, \mathrm{ax}}=\frac{0.67 \cdot \bar{\lambda}}{n-\sqrt{n^{2}-\mathrm{NA}^{2}}}
\end{gathered}
$$

$n$ displays the refractive index of the immersion medium and $\bar{\lambda} \approx \sqrt{\lambda_{\mathrm{ex}} \cdot \lambda_{\mathrm{em}}} \cdot{ }^{[142,147]} \mathrm{In}$ the setup used in this work this leads to a lateral resolution in a range of $200-300 \mathrm{~nm}$ and an axial one of about 700-1000 nm.[148]

Resolution is still a topic of current interest as the ongoing development of new techniques or resolution improvements show. During this work the possibility was created to analyze fluorescently labeled specimen using the Airyscan technology. 


\subsubsection{The Airyscan Technology}

Airyscan is a new feature which enables an improved signal-to-noise ratio and superresolution for confocal scanning laser microscopes. Airyscan by itself is a detector concept which provides 32 channel area detectors instead of a single point detector.[149] This area detector is collecting all the light of an airy pattern (illustrated in Figure $3.16 \mathrm{~B} / \mathrm{C}$ ), and simultaneously this arrangement converts each detector into a single, very small pinhole. Thus, the signal-to-noise ratio as well as the resolution of the collected objective is improved.

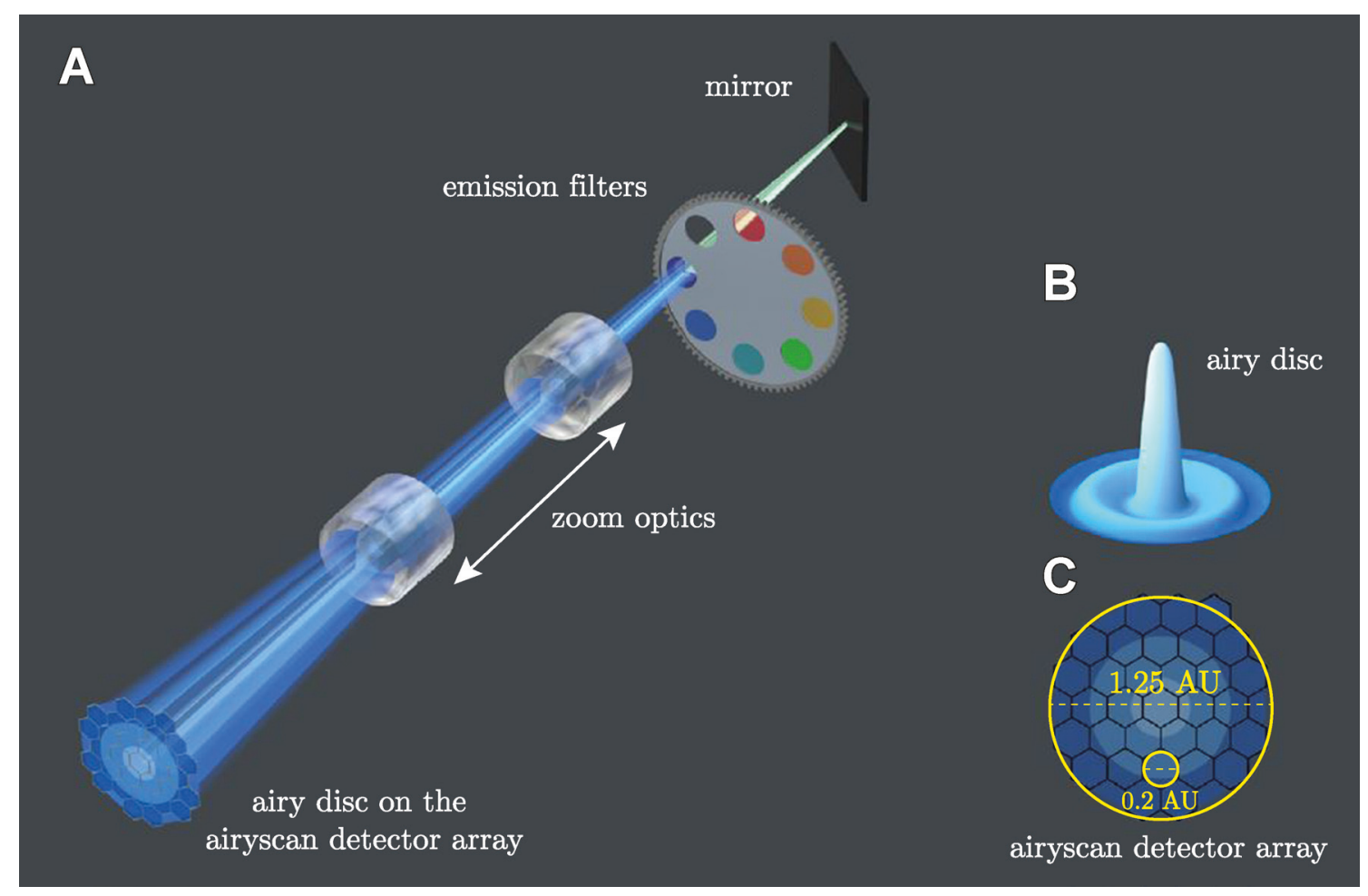

Figure 3.16: Illustration of Airyscan principle. A: Beam path of the Airyscan detector. Light is reflected towards the detector by a mirror and passing through an emission filter. Zoom optics focus the resulting airy disc. B-C: The airy disc (shown in 3D, B) is collected by the Airyscan detector array $(\mathbf{C})$. All photons relating to the airy disc are used as the whole airy disc is collected by the array (C). Modified from Carl Zeiss Microscopy GmbH, Airyscan brochure. ${ }^{[150]}$

As shown above, an Airyscan detector increases the detection efficiency significantly, as this detector array is covering around $1.25 \mathrm{AU}$, importantly more than $1 \mathrm{AU}$ to not reject some light as one pinhole does. Each detector element produces its own image which leads to a sectioning and resolution in $x, y$ and $z$ of $0.2 \mathrm{AU}$, whereas the sensitivity sticks to 1.25 AU (Figure 3.16 C).[151] Then, shifting all images back to the central position which can be done due to the fact that the displacements are known by calibration, and summing up the back shifted signal produces an image (a so-called 
"Sheppard sum") with an already higher resolution of about factor 1.4 compared to a classical confocal image.[151-153] Subsequent deconvolution by ZEN software (Carl ZeisS Microscopy GmbH, Oberkochen, Germany) is further boosting the resolution information.[151]

In this work small actin filaments labeled with Alexa Fluor ${ }^{\circledR}$ 488-phalloidin were imaged with Airyscan. At the wavelength of $488 \mathrm{~nm}$ the resolution is improved to $140 \mathrm{~nm}$ laterally and $400 \mathrm{~nm}$ axially using Airyscan. ${ }^{[153-156]}$ An exemplary comparison of classical confocal image to an Airyscan image of this work is shown in Figure 3.17.
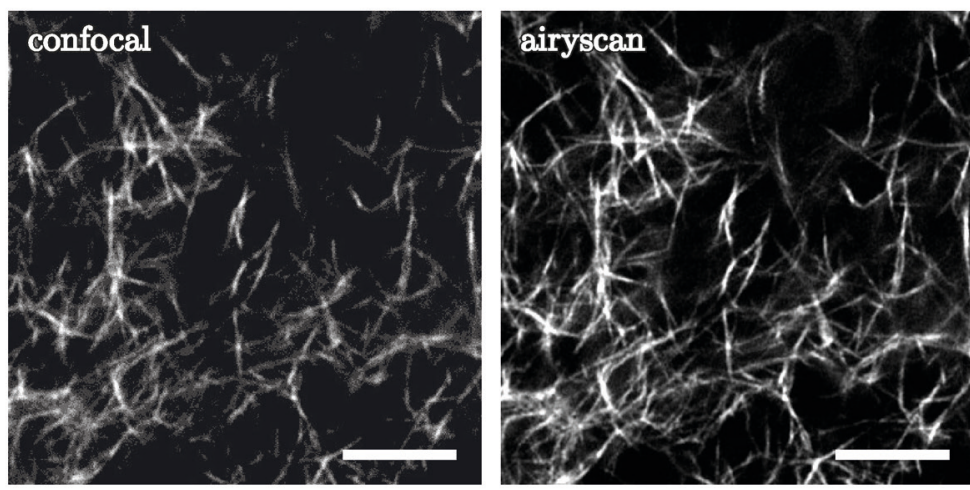

Figure 3.17: Exemplary comparison of a confocal and an Airyscan image of a typical 3D F-actin gel. The left image was obtained using a GaAsP-PMT detector of a Zeiss LSM 880. The pinhole was set to collect 1.25 AU for comparison. The right image is the same spot of the 3D F-actin gel utilizing the Airyscan detector of the LSM 880. A clear resolution improvement is observable. Scale bar: $10 \mu \mathrm{m}$.

In this work two different confocal laser scanning microscopes were used. First, a FluoView 1200 (Olympus Europe SE \& Co.KG, Hamburg, Germany) equipped with 60x (LPUMPlan $\mathrm{N}, \mathrm{NA}=1.0$, Olympus) or 40x (LPUMPlanFL $\mathrm{N}, \mathrm{NA}=0.8$, Olympus) water immersion objective was used. Second, an LSM 880 (Carl ZeisS Microscopy $G m b H$, Oberkochen, Germany) with an Airyscan detector equipped with a 40x (W Plan-Apochromat, NA = 1.0, Zeiss) objective was used after August 2017. 


\subsubsection{Fluorescence Recovery after Photobleaching (FRAP)}

Fluorescence recovery after photobleaching (FRAP) is a method often used to determine two-dimensional diffusion coefficients in cells or other microbiological setups. In this work FRAP experiments are employed to determine the diffusion coefficient of the lipid bilayer's lateral mobility. The general principle of FRAP experiments is illustrated in Figure 3.18. Fluorescently labeled lipids, in case of this work TxR-DHPE, are bleached in a specific region of interest (ROI) due to a powerful laser pulse (Figure $\left.3.18 \mathrm{~B}, t_{0}\right)$. In the tracked readout intensity of this ROI a decrease in intensity can be observed (Figure 3.18 D). Because of the lateral mobility of the lipid bilayer bleached labeled lipids leave the ROI while fluorescently labeled lipids out of the ROI, therefore not bleached, diffuse into. The tracked fluorescence intensity of the ROI increases again until a limiting fluorescence level is reached (I, Figure $3.18 \mathrm{C}$ ).

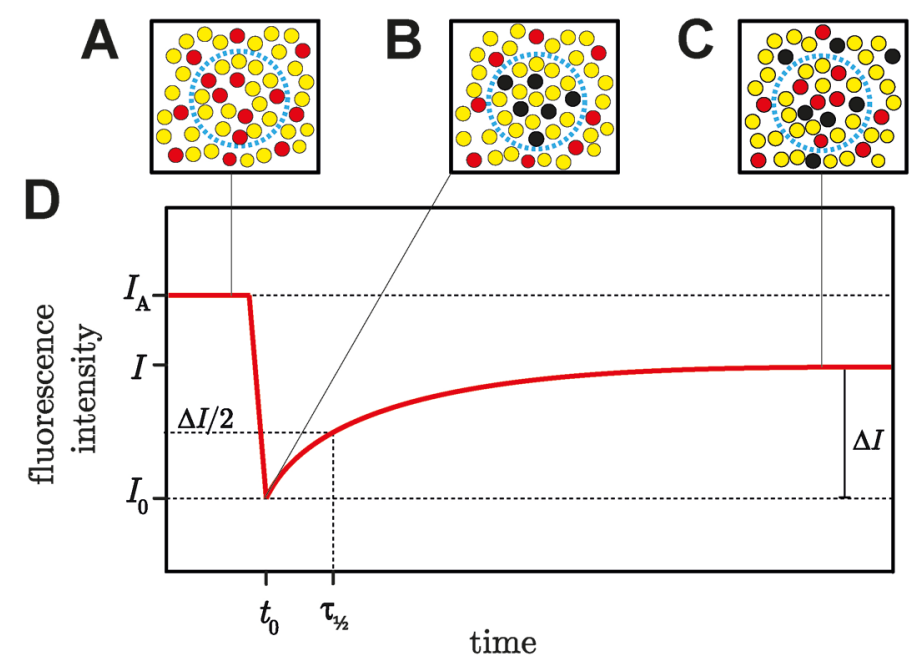

Figure 3.18: Schematically representation of a FRAP experiment. A: A specific region of interest (ROI, cyan) is used to determine the fluorescence of this spot (D). Non-labeled lipids (yellow) as well as fluorescently labeled lipids (red) are homogeneously distributed. B: The fluorescence inside the ROI is bleached using a laser pulse and the fluorescently labeled lipids are bleached (black). C: Due to lateral mobility of the lipid bilayer bleached vesicles leave the ROI and labeled lipids diffuse into the ROI. D: The readout fluorescent intensity is plotted against the experiment duration. An intensity decrease from the starting point $\left(I_{\mathrm{A}}\right)$ to the lowest intensity level occurs $\left(I_{0}\right)$ and a recovery of the intensity until a limiting level $(I)$ can be observed.

Using the time resolved recovery of the fluorescence intensity the diffusion coefficient can be determined. The first approach of quantifying the diffusion coefficient $(D)$ was reported by Axelrod et al. utilizing Fick's second law (equation 3.21).[157]

$$
D=\frac{r^{2}}{4 \cdot \tau_{D}}
$$


$\tau_{D}$ is the characteristic diffusion time which can be determined by an exponential description of the curve progression shown in Figure $3.18 \mathrm{D}$ and $r$ is the radius of the bleached spot. To pursuit this approach, some assumptions were made: First, the profile of the bleaching spot has to be in Gaussian shape, the bleach pulse must be short in contrast to the recovery time and photobleaching is described as an irreversible first-order kinetic. ${ }^{[158]}$ To overcome some of these assumptions, a Hankel transform of the recorded data is used, which does not rely on the bleaching geometry. Furthermore, it provides advantages regarding temporal variations in intensity and a decreased influence of noise in comparison to earlier described FRAP analysis methods. Due to these reasons, a Hankel transform method provided as MATLAB script and published by Jönsson et al. was used in this work to determine diffusion coefficients.[159] 


\subsection{Data Evaluation}

\subsubsection{Skeletonization of Network Images}

One main subject of this work is the evaluation of the self-organization of filamentous actin networks. The main result of how F-actin self-organizes within a gel or during attachment is the network structure. The morphology of these networks is obtained from fluorescence micrographs. These micrographs mostly suffer from different and low signal-to-noise ratios, low contrasts and intensity fluctuation along the filaments. Simplistic analysis methods like contrast enhancement with a subsequent thresholding for example mostly fail to reproduce the network structures. The human eye is still able to detect the filamentous structure while the threshold interrupts the structure. To overcome these challenges two techniques recently presented in literature were applied to the obtained micrographs. The expected outcome is a network skeleton which then can be further evaluated to obtain characteristic, quantitative parameters to describe the network morphology.

\subsubsection{Skeletonization via Artificial Retina}

- Image processing via 'Artificial Retina' has been developed by Nils Dörrer as part of his bachelor thesis -

- Retina analysis has been published in "Rheology of Membrane-Attached Minimal Actin Cortices" by H. Nöding, M. Schön, C. Reinermann, N. Dörrer, A. Kürschner, B. Geil, I. Mey, C. Heussinger, A. Janshoff and C. Steinem, J. Phys. Chem. B, 2018, 122, 4537-4545 [160] -

In a first step the obtained 2D fluorescence micrograph was subjected with a contrastlimited adaptive histogram equalization (CLAHE) to obtain an intensity equilibrated image with full intensity range. The local contrast in distinctive sections of the image is used to redistribute the intensity levels of the image. To not overamplify the noise in homogeneous regions the amplification is limited by the contrast.

The main goal of image processing is to classify each pixel to filament or background. To overcome a simple threshold, the neighborhood of each pixel is considered to get more information about the environmental structure. This was realized using a LoG-filter bank (Laplace of Gaussian) constructed by $N_{\mathrm{f}}$ centered, pole-like filamentous motives ( $N_{\mathrm{f}}$ displays the number of different motives). These motives were usually in a range of $21 \times 21$ pixels up to $81 \times 81$ pixels used for images with a size of 1024x1024 pixels. 
Furthermore, they display intensity profiles which are shown in Figure 3.19 B and are modeled by the Mexican-Hat (equation 3.22) function, which is the Laplacian of the Gaussian (LoG).

$$
I(x, y)=\frac{1}{\pi \cdot \sigma^{2}}\left(1-\frac{1}{2} \cdot \frac{x^{2}}{\sigma^{2}}\right) \cdot e^{-\frac{x^{2}}{\sigma^{2}}}
$$

In this case $\sigma$ controls the thickness of the poles as illustrated in the inlet of Figure 3.19 A. The usage of differentiations of Gaussian normal distributions is a common method for structural analysis of images. ${ }^{[161]}$

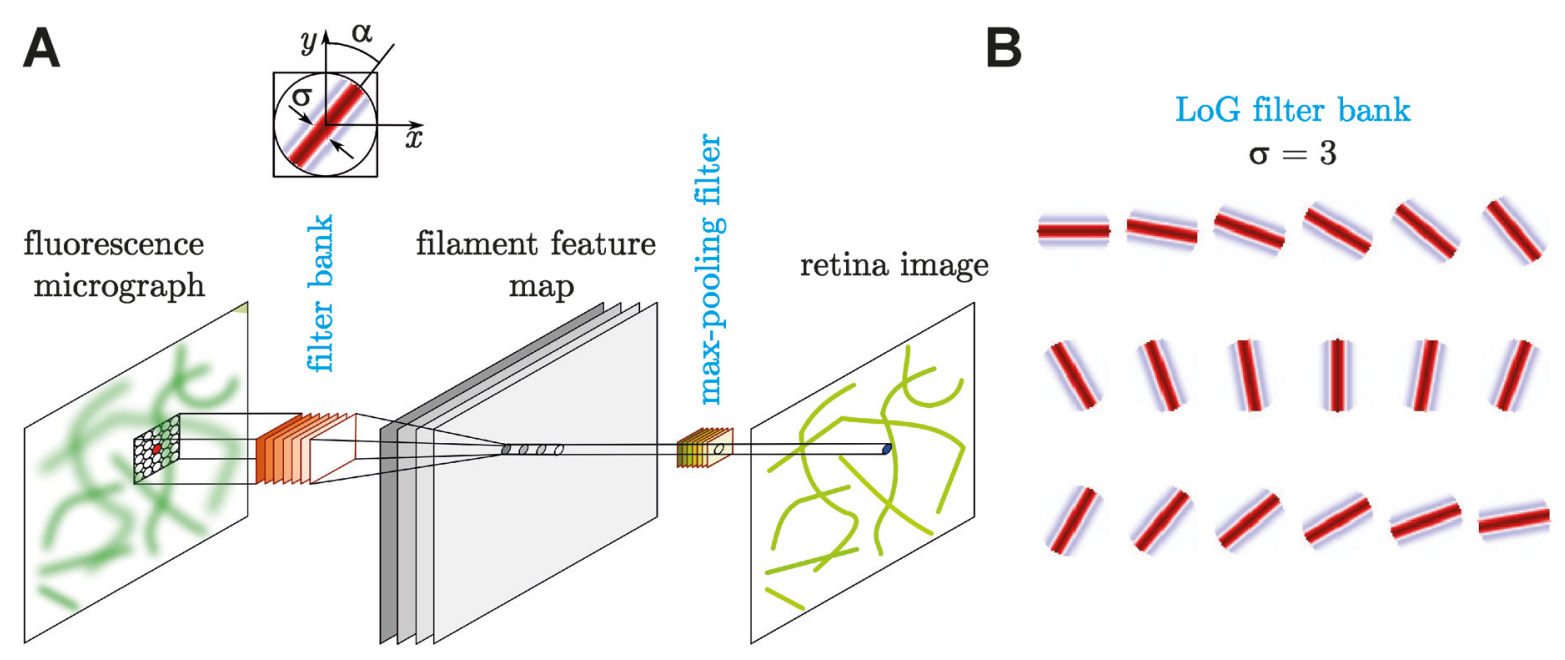

Figure 3.19: Concept of image processing using the artificial retina analysis. ${ }^{[160]} \mathrm{A}$ : Every pixel of a fluorescence micrograph is analyzed in dependence of his surrounding neighborhood. It gets transacted to a filament feature vector using a filter bank. In the inlet the parameters of a single filter bank motif are shown. One exemplary LoG filter bank for 18 rotational steps and a constant $\boldsymbol{\sigma}=3$ is shown in (B). A max-pooling filter, a sample-based discretization process, is used to reduce the dimensionality and create the retina image. Modified and reprinted with permission from Nöding, Schön et al. Copyright 2018 American Chemical Society. ${ }^{[160]}$

The achieved motives are then rotated between $0^{\circ}$ and $170^{\circ}$ to cover possible pole orientations. Typically, orientations were covered in $10^{\circ}$ steps leading to 18 possible filters for one filament thickness $\sigma$. An optimal range for the parameter $\sigma$ was experimentally found and is dependent on the image resolution. Most of the time, a filter bank contains 8 to 10 different $\sigma$-values, leading to a filter bank containing around $N_{\mathrm{f}} \approx 150$ different motives. It turned out that these filters are prone to anisotropic artefacts: poles with a $45^{\circ}$ orientation are longer than other poles. This led to diagonal, stripe-like intensity artefacts in the filter response images. Therefore, every motive was masked with a circle exactly fitting into the square motive and setting the outside intensity to zero. A final refinement was done by normalizing all motives to an integral intensity of zero. In total, every pixel is described with a $N_{\mathrm{f}}$-dimensional feature vector with information about the character and the environment of it. A convolution of these 
feature vectors that encode the information of the local network structure is then used to visualize the resulting information. Therefore, a final reduction of the feature vectors is done. The filament- or background-character of a pixel is obtained by interpreting the value of the largest feature vector component as the resulting intensity. This emphasizes the filaments smoother and with less noise than in the raw image. This resulting image is called retina image (Figure $3.20 \mathrm{~B}$ ) as the retina of mammal eyes also reduces the signal of many sensors to one single image. This retina image is then suited for employing an intensity threshold, followed by skeletonization (Figure 3.20 C). As every pixel is analyzed by its neighborhood, a frame at the image border occurs. This frame must be subtracted for further analysis.
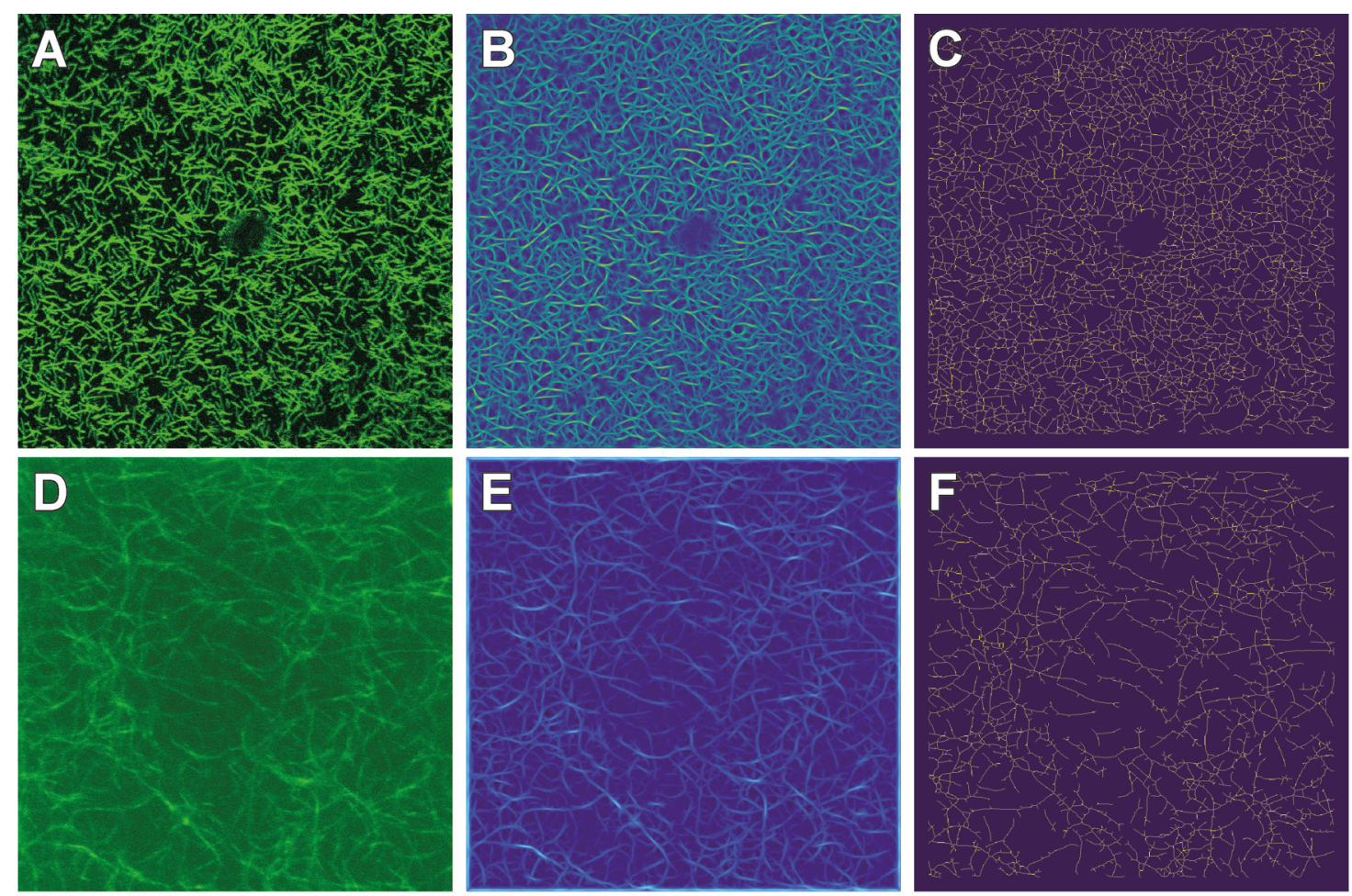

Figure 3.20: Exemplary skeletonization of two fluorescence micrographs with different signal-to-noise ratios via the artificial retina. A-C: A typical fluorescence micrograph $(\mathbf{A})$ is subjected to a retina image $(\mathbf{B})$ using the artificial retina analysis. This image is then skeletonized $(\mathbf{C})$ utilizing an intensity threshold. D-F: A fluorescence micrograph with a lower signal-to-noise ratio $(\mathbf{D})$ is subjected to a retina image $(\mathbf{E})$. However, the resulting retina image shows clear filaments and is almost noise free. The image borders show artefact due to missing neighbor pixels (E). Therefore, the skeleton is framed $(\mathbf{F})$ and does not consider the image borders. 


\subsubsection{Skeletonization via SOAX}

A further skeletonization approach was the use of the software SOAX, which was developed by $X u$ et al. to quantify and evaluate the topology and geometry of dense 3D networks. [162] This method was proposed to extract the network information of a $3 \mathrm{D}$ actin meshwork which were imaged by stacks of confocal microscopy.[163] The software needs input arguments or parameters which were demonstrated by $\mathrm{Xu}$ et al. and were found empirically.[163] The basic method is the initializing and evolution of stretching open active contours (SOACs) as illustrated in Figure 3.21.

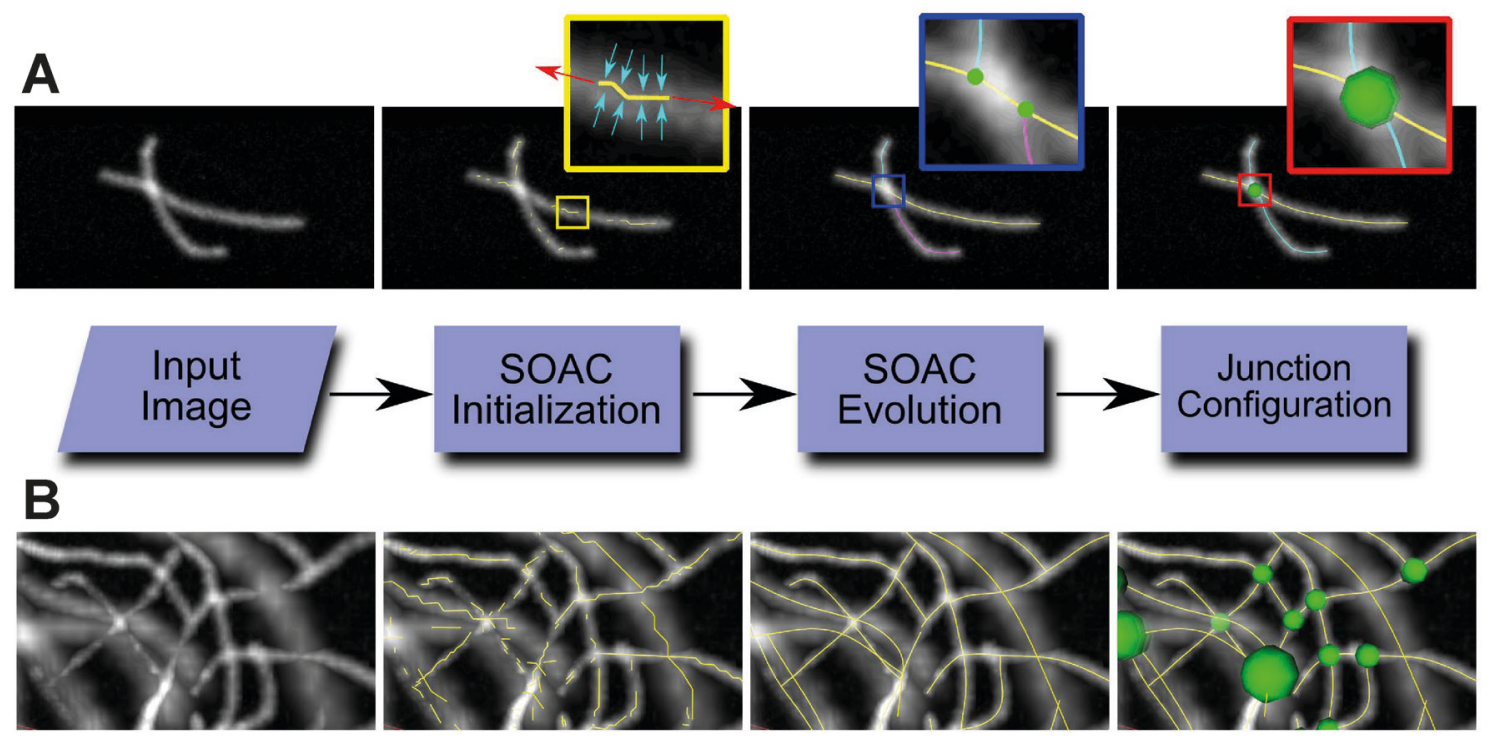

Figure 3.21: Overview of the operating principle of the software SOAX. Scheme is subdivided in four columns of operation steps. First, the input image is given. Second, SOACs are initialized based on the input arguments. Third, SOACs are evolved and merged to obtain a network. Fourth, Junctions (green spheres) are configurated and highlighted. A: Results of every operating step on a simple two filament image. The yellow magnification square shows the forces exerted on a SOAC during evaluation process (cyan arrows). The blue magnification square displays T-junctions of sequential evolved SOACs (green spheres) while the red one shows the reconfigured filament junction. B: The whole process illustrated on a more complex network system. Adapted, in the image order, from $X u$ et al. (work is licensed under Creative Commons Attribution 4.0 International License). ${ }^{[162]}$

The three basic steps of SOAX are: SOAC initialization, SOAC evolution and junction configuration (Figure 3.21 A). The SOAC is simply a parametric curve which is evolved further by stretching in form of elongation till it reaches the tip, the filament end. Meanwhile the SOAC is attracted to the centerline of the filament as shown in the yellow magnification box (cf. Figure $3.21 \mathrm{~A}$ ). The first step, the initialization step, multiple short SOACs (parameter dependent) are placed at intensity ridges of the image which correspond to filament centerlines. Subsequently, these initialized SOACs are evolved by stretching accordingly to the local intensity contrast. The elongation is controlled by a global parameter $k_{\text {str }}$. The evolution is stopped when the SOAC reaches 
a filament end or hits another SOAC. In the second case a T-junction is formed as shown in the blue magnification box (cf. Figure $3.21 \mathrm{~A}$ ). In this example the cyan and magenta colored SOAC hit the yellow SOAC and form two T-junctions. The third and last stage of analysis is clustering of nearby T-junctions into one single junction. Then, the local network connectivity of the SOACs is configured by cutting and slicing the SOACs to avoid ending or sharp bending at junctions (cf. Figure 3.21 A, red box). The obtained re-configured SOACs with combined T-junctions are used as skeleton which represent the topology of the imaged network.

\subsubsection{Node Density Analysis}

- Node density analysis was performed using a PYTHON script of Nils Dörrer developed in his bachelor thesis -

Using the obtained network skeletons, quantitative network parameter should be determined. The detection of intersections or branching points, together defined as nodes, is important to divide filaments in segments and to determine a specific density of nodes per area, the so-called node density. Since skeletons are described with a width of 1 pixel, an analysis regarding to single pixels was used. The skeletonization algorithm reduces the filament to a chain of pixels, therefore every pixel has two neighbor filament-pixels except capping pixel with only one neighbor filament-pixel. Thus, a single filament-pixel is then determined as a node pixel when it has more than two active neighbor filament-pixels as illustrated in Figure 3.22 A. It must be considered that at one intersection more than one pixel can have 3 or more neighbors as illustrated in Figure 3.22 B. In this case, these collections of nodes had to be reduced to one single, real node. The number of determined nodes of one image is then related to the image size which leads to several nodes per area, in case of this work square micrometer $\left(\mu^{2}\right)$.
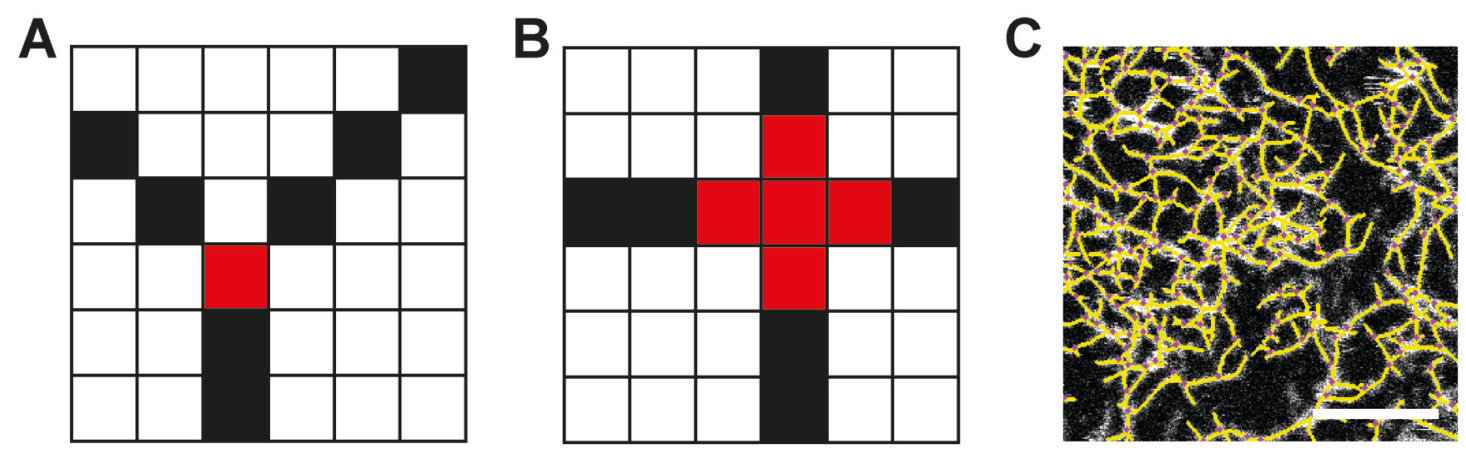

Figure 3.22: Schematic pixel diagrams of node determination and resulting skeleton with highlighted nodes. A: Pixel diagram showing background pixel (white), filament pixel (black) and determined node pixel (red) with three or more filament pixels in its 8-pixel neighborhood. B: In many cases there are a few pixels close to each other having three or more 
neighbors. In this example all five red marked pixels have four filament pixels in their 8-pixel neighborhood. This will be subsequently reduced to one node pixel. C: The resulting skeleton (yellow) illustrated on a black and white fluorescence micrograph with detected nodes highlighted in magenta.

\subsubsection{Mesh Size Analysis}

The used mesh size analysis is based on the work of Molteni et al. who described a " $2 D$ bubble analysis of biopolymer filamentous networks pore size" to obtain the average pore or mesh size $\xi$ of in silico fibrin gels. ${ }^{[164]}$ Their determination of the mesh size is based on placing circles with a maximum diameter inside network pockets. The procedure to find the circle center location is computed using an iterative procedure which converges to a maximum coverage by circle area of the $2 \mathrm{D}$ image. This method was simplified by Münster and Fabry as they showed that using an Euclidian distance map (EDM) reduces the algorithm to a two-step procedure.[165] On the basis of a skeletonized image with filament-pixels (value 1) and the pocket areas (value 0), the EDM is computed for the pocket areas and assigns the distance of each pixel (0) to the nearest filament pixel (1). The created EDM then provides local maxima which determine the center of a circle with the largest diameter without overlapping any filament-pixels. As the network pockets demonstrate many different shapes it is possible that one network pocket creates many circles which overlap inside one pocket (Figure $3.23 \mathrm{~A}$ ). A comparison of overlapping circles is implemented and only the circle with the largest diameter remains inside the network pocket (Figure $3.23 \mathrm{~B}$ ). This circle is decisive for the mesh size $\xi$ of the pocket. The computation of EDM, determination of local maxima and comparison of overlapping circles was implemented in a MATLAB script written by Dr. Ingo P. Mey (Georg-August-Universität Göttingen). Script is attached in the appendix (cf. chapter 7.4.1).
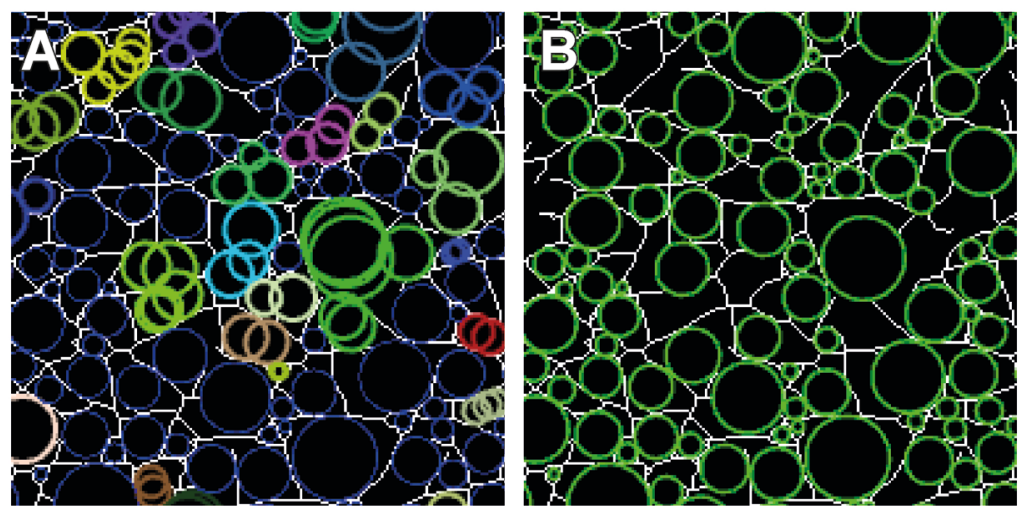

Figure 3.23: Important intermediate steps of the mesh size analysis. A: After computation of the EDM local maxima determine circle centers (multicolored) with a maximum diameter to the filamentous network structure (white). Overlapping circles inside one pocket are shown in one color determined as one cluster of circles. B: A comparison of all clustered circles leaves only one circle per network pocket (green) which is decisive for the mesh size of the pocket. 


\subsubsection{Video Particle Tracking}

- VPT was implemented in cooperation with Helen Nöding as part of project A17 of the collaborative research center $937^{[166]}$

Video particle tracking (VPT) is a passive microrheological force spectroscopy method which was used to determine the frequency dependent viscoelastic properties of MACs (minimal actin cortices). This method is widely used in literature to probe artificial model systems, such as cross-linked or entangled F-actin gels. In this work VPT was used to record the viscoelastic properties of F-actin networks mediated by ezrin to lipid bilayers attached, as well as 3D F-actin gels. One requirement for that is the incorporation of tracer particles inside the F-actin network. F-actin was polymerized in presence of carboxylate-modified polystyrene beads (cf. chapter 3.3.5, $\varnothing=2 \mu \mathrm{m}$ ) to incorporate these beads in the actin meshwork (cf. chapter 3.3.5). Then, MACs were prepared in the same way as MACs without tracer particles (cf. chapter 3.3.6). After an equilibration time of at least two hours, a time series of at least 100,000 images was recorded using a scientific complementary metal-oxide-semiconductor (sCMOS) camera (Zyla 5.5-CL-10, Andor Technology Ltd., Belfast, UK). This camera was mounted onto an Olympus FluoView 1200 CLSM with a 60x objective (cf. chapter 3.5.2). A total of 1-10 beads could be recorded in one frame. Exposure time was about $10 \mathrm{~ms}$ and images were recorded with a frame rate of at least 50 frames per second.

For temperature dependent measurements, a home-built Peltier element mounted to the CLSM setup (built by Johannes Schumacher, Georg-August-Universität Göttingen) was used, which was able to adjust the temperature at the specimen's bottom plate as well as at the objective. Temperature was controlled within a margin of less than a tenth degree Celsius by utilizing a proportional-integral-derivative controller.

\subsubsection{Data Processing}

- VPT was evaluated by Helen Nöding as part of project A17 of the collaborative research center $937^{[166]}$

The typical evaluation pathway of VPT measurements is illustrated in Figure 3.24. The previously recorded time (cf. chapter 3.6.4) series, showing displacements of the tracer particles, is the raw data (cf. Figure $3.24 \mathrm{~A}$ ). Particle tracking, calculation of the mean squared displacements (MSDs) and determination of the frequency dependent shear moduli was conducted using a MATLAB script written by Maria Kilfoil.[167] This script was based on the theory of Weitz et al. and included a tracking algorithm of 
John Crocker. ${ }^{[168]}$ Bead tracking with a subpixel resolution of $3 \mathrm{~nm}$ was possible in the setup of this work (cf. Figure 3.24 B, inlet). Particle trajectories, which expand less at MACs in comparison to 3D F-actin gels (cf. Figure 3.24 B) were checked for artefacts before further analysis, and those with sudden jumps, sharp changes in position or obvious artefacts over time were rejected. The MSD of one bead trajectory was ensemble averaged additionally to the rather high time average of at least 50,000 time points. [166]
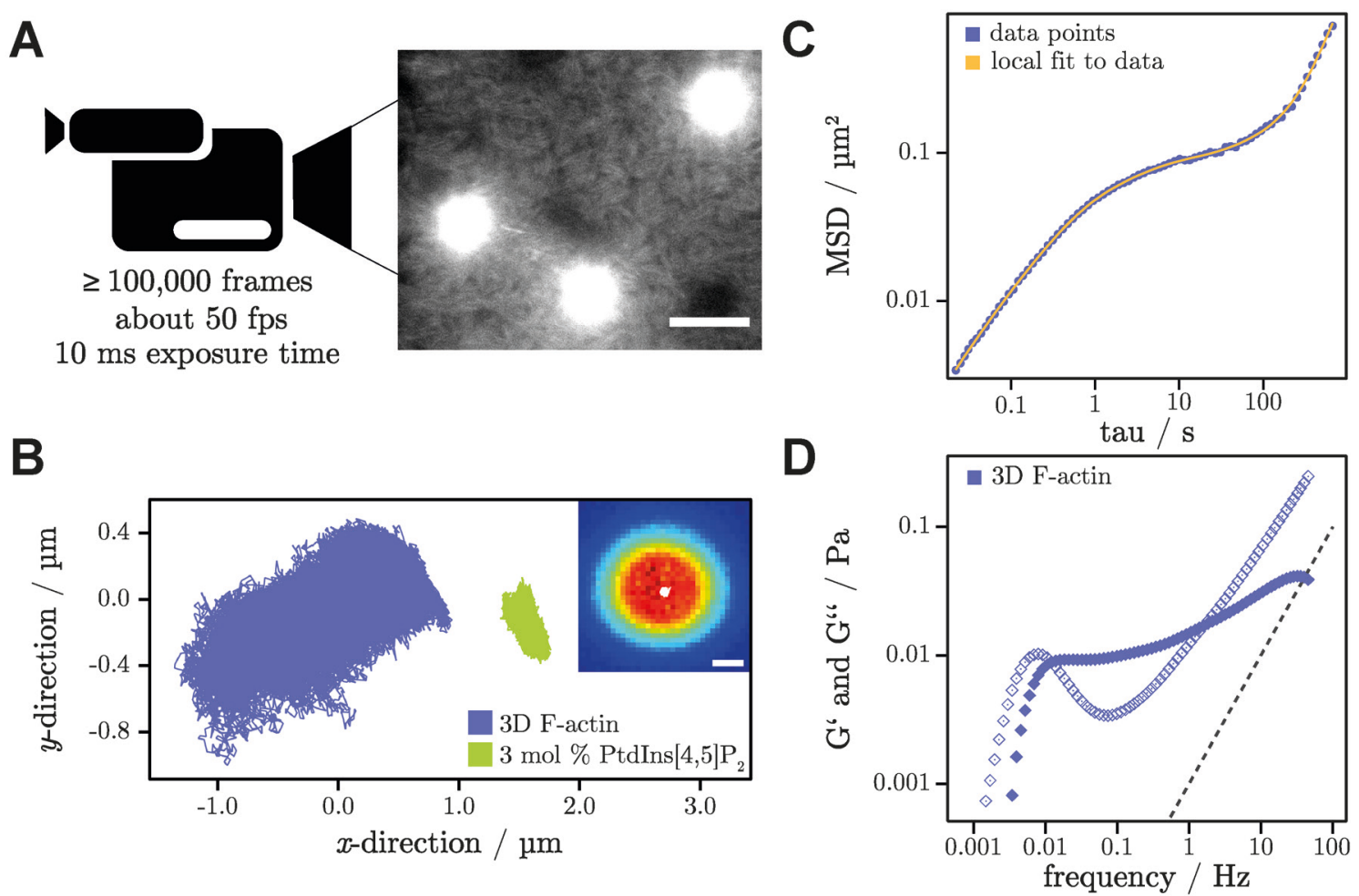

Figure 3.24: Overview of video particle tacking microrheology. A: Schematic illustration of VPT measurement: a time series of bead movement was recorded using a sCMOS camera and displayed settings. B: Exemplary trajectories of tracer particles incorporated in the viscoelastic media of a 3D F-actin gel as well as of a 2D F-actin cortex attached to a lipid bilayer containing $3 \mathrm{~mol} \% \mathrm{PtdIns}[4,5] \mathrm{P}_{2}$ are shown. C: The mean squared displacement and the respective local fit are displayed. D: The resulting viscoelastic properties are shown as storage ( $G$ ', filled symbols) and loss modulus ( $G$ ', open symbols). The dotted line displays the theoretical curve for a tracer particle in water. Scale bars: A: $4 \mu \mathrm{m}, \mathrm{B}: 1 \mu \mathrm{m}$. Graphic was kindly provided by Helen Nöding. ${ }^{[166]}$

The MSD was fitted locally with a second-degree polynomial, which was subsequently used to calculate the viscoelastic moduli (cf. Figure $3.24 \mathrm{C}$ ). Briefly, the relation of the complex shear modulus and the Laplace transform of the MSD $\left\langle\Delta \tilde{x}^{2}(s)\right\rangle$ is established (equation 3.23) by connecting the generalized Stokes-Einstein equation and the relation of the complex viscosity $\tilde{\eta}(s)$ and the shear modulus $\tilde{G}(s) . s$ shows the frequency parameter in the Laplace domain. 


$$
\tilde{G}(s)=\frac{N \cdot k_{\mathrm{B}} T}{3 \pi r \cdot s \cdot\left\langle\Delta \tilde{x}^{2}(s)\right\rangle}
$$

In Fourier space:

$$
G^{*}(\omega)=\frac{N \cdot k_{\mathrm{B}} T}{3 \pi r \cdot i \omega \cdot\left\langle\Delta \tilde{x}^{2}(\omega)\right\rangle}
$$

Here, $N$ is the number of dimensions, $k_{\mathrm{B}}$ is the Boltzmann constant, $T$ the temperature, $r$ the bead radius and $\left\langle\Delta \tilde{x}^{2}(\omega)\right\rangle$ the Fourier transform of the MSD, where $s$ equals $i \omega .{ }^{[166]}$ The complex shear modulus $G^{*}(\omega)$, hence the storage modulus $G^{\prime}(\omega)$ and the loss modulus $G^{\prime \prime}(\omega)$, were determined using an alternative form of the generalized Stokes-Einstein equation derived by Dasgupta et al. based on the method introduced by Mason et al. (cf. Figure 3.24 D).[166,168,169]

The obtained frequency-dependent viscoelastic properties can be interpreted using the semi-flexible polymer theory developed by Morse, Gittes and MacKintosh or Heussinger. ${ }^{[170-173]}$ In general, the elastic response of entangled semi-flexible polymers, such as F-actin, to an applied strain arises from the stretching of filaments. Exemplary frequency dependent viscoelastic properties, illustrated as schematic curve progressions of loss (blue line) and storage moduli (red line), are shown in Figure 3.25. Typically, three different frequency regimes within the viscoelastic behavior of purely entangled networks are distinguished: The low frequency regime (I, blue) which is dominated by filament diffusion such as reptation and transient dissociation, the plateau region (II, green) which is dominated by elastic behavior due to filament entanglement and the high frequency (III, yellow) dominated by bending of single filaments.

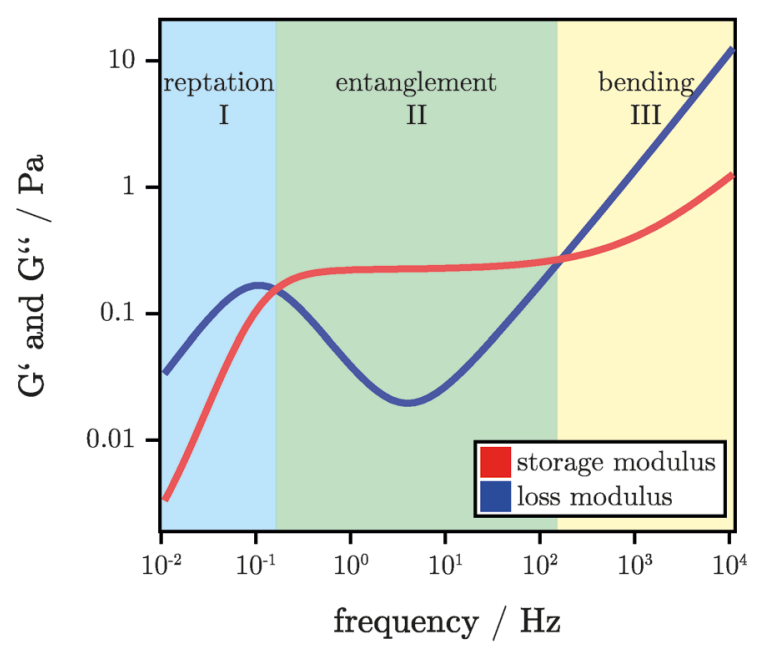

Figure 3.25: Schematic illustration of frequency dependent viscoelastic properties of semi-flexible polymers such as F-actin. Three frequency regimes are distinguished: The low frequency regime (I), dominated by reptation movements of filaments, the plateau regime (II), dominated by entanglements and the high frequency regime (III), dominated by bending modes. Regimes are in each case separated by cross-overs of storage (red) and loss modulus (blue). Graphic was kindly provided by Helen Nöding. ${ }^{[166]}$ 


\section{Results and Discussion}

\subsection{Characterization of Ezrin Binding as a Function of Pinning Point Density}

The protein ezrin is known to bind the receptor lipid L- $\alpha$-phosphatidylinositol4,5-bisphosphate (PtdIns[4,5] $\left.\mathrm{P}_{2}\right)$. The density of the supplied binding sites from PtdIns $[4,5] \mathrm{P}_{2}$ is referred to as pinning point density.

\subsubsection{Ezrin T567D Plasmid Isolation}

First, the quality of the bacterial plasmid had to be checked by its isolation and sequencing as described in chapter 3.2.2 and 3.2.3. A missing of a possible point mutation could thus be prevented. Plasmid sequencing was done at irregular intervals but at least once a year. The Sanger sequencing was carried out by Seqlab, Inc. Polymerization of the plasmid was induced by the two primers T7 promoter and T7 terminator. ${ }^{[174]}$ With $\mathrm{T} 7$ promoter as a forward primer and $\mathrm{T} 7$ terminator as a reverse primer an antiparallel overlay led to the complete base sequence of the plasmid. This gathered base sequence was translated to an amino acid sequence via the Protparam tool by ExPASy.[175] The translated decryption was crosschecked with the original amino acid sequence of the wild type protein as shown in Figure 4.1.[176] Only the desired point mutation at position 567, where threonine is substituted by an aspartate, was observed in every case.

\begin{tabular}{|c|c|c|c|c|c|c|c|}
\hline & 1 & 11 & 21 & 31 & 41 & 51 & \\
\hline & I & 1 & I & I & I & 1 & \\
\hline \multirow[t]{2}{*}{1} & MPKPINVRVT & TMDAELEFAI & QPNTTGKQLF & DQVVKTIGLR & EVWYFGLHYV & DNKGFPTWLK & 60 \\
\hline & MPKPINVRVT & TMDAELEFAI & QPNTTGKQLF & DQVVKTIGLR & EVWYFGLHYV & DNKGFPTWLK & \\
\hline \multirow[t]{2}{*}{61} & LDKKVSAQEV & RKENPLQFKF & RAKFYPEDVA & EELIQDITQK & LFFLQVKEGI & LSDEIYCPPE & 120 \\
\hline & LDKKVSAQEV & RKENPLQFKF & RAKFYPEDVA & EELIQDITQK & LFFLQVKEGI & LSDEIYCPPE & \\
\hline \multirow[t]{2}{*}{121} & TAVLLGSYAV & QAKFGDYNKE & VHKSGYLSSE & RLIPQRVMDQ & HKLTRDQWED & RIQVWHAEHR & 100 \\
\hline & TAVLLGSYAV & QAKFGDYNKE & VHKSGYLSSE & RLIPQRVMDQ & HKLTRDQWED & RIQVI & \\
\hline \multirow[t]{2}{*}{181} & GMLKDNAMLE & YLKIAQDLEM & YGINYFEIKN & KKGTDLWLGV & DALGLNIYEK & DDKL & 2 \\
\hline & GMLKDNAMLE & YLKIAQDLEM & YGINYFEIKN & KKGTDLWLGV & DALGLNIYEK & DDKLTPKIGF & \\
\hline \multirow[t]{2}{*}{241} & PWSEIRNISF & NDKKFVIKPI & DKKAPDFVFY & APRLRINKRI & LQLCMGNHEL & YMRRRKPDTI & \\
\hline & PWSEIRNISF & NDKKFVIKPI & DKKAPDFVFY & APRLRINKRI & LQLC & KPDTI & \\
\hline \multirow[t]{2}{*}{301} & EVQQMKAQAR & EEKHQKQLER & QQLETEKKRR & ETVEREKEQM & MREKEELMLR & LQDYEEKTKK & 360 \\
\hline & EVQQMKAQAR & EEKHQKQLER & QQLETEKKRR & ETVEREKEQM & MREKE & KTKK & \\
\hline \multirow[t]{2}{*}{361} & AERELSEQIQ & RALQLEEERK & RAQEEAERLE & ADRMAALRAK & EELERQAVDQ & IKSQEQLAAE & 420 \\
\hline & AERELSEQIQ & RALQLEEERK & RAQEEAERLE & & EELERQAVDQ & IKSQEQLAAE & \\
\hline \multirow[t]{2}{*}{421} & LAEYTAKIAL & LEEARRRKED & EVEEWQHRAK & EAQDDLVKTK & EELHI & PPPPPPVYEP & 400 \\
\hline & LAEYTAKIAL & LEEARRRKED & EVEEWQHRAK & EAQDDLVKTK & EELHLVMTAP & PPPPPPVYEP & \\
\hline \multirow[t]{2}{*}{481} & VSYHVQESLQ & DEGAEPTGYS & AELSSEGIRD & DRNEEKRITE & AEKNERVQRQ & LLTLSSELSQ & \\
\hline & VSYHVQESLQ & DEGAEPTGYS & AELSSEGIRD & DRNEEKRITE & AEKNERVQRQ & LLTLSSELSQ & \\
\hline \multirow[t]{2}{*}{541} & ARDEN & DI I HNENMRQ & RDKYKTLRQ & IRQGNTKQRI & +2 & & \\
\hline & DENKRTHN & DI I HNENMRQ & RDKYKDLRQ & RQGNTKQRI & EFEAL & & \\
\hline
\end{tabular}

Figure 4.1: Comparison of determined amino acid sequence (1-letter code) with sequence of UniProt library. ${ }^{[176]}$ In blue received data from Seqlab, Inc. are shown and 
compared to the sequence of the UniProt library (black). Every amino acid is in accordance, expect the desired point mutation of threonine 567 to aspartate (highlighted in yellow). At Position 117 and 284 the cysteine residues are positioned which will be addressed later.

\subsubsection{Isolation of Ezrin T567D}

Isolation and purification of the protein ezrin's active mutant T567D was carried out several times as already specified in chapter 3.2.4 and 3.2.5, and was done in analogy to previous studies. ${ }^{[116,131,177,178]}$ Each isolation was controlled by analyzing samples of isolation steps with SDS-PAGE (chapter 3.2.6). An exemplary image of an SDS-Gel is shown in Figure 4.2. SDS-PAGEs only allow a qualitative classification, that is why a low molecular mass standard (Figure 4.2, left line) was needed for interpretation. A band running at around $80 \mathrm{kDa}$ is predominant in every analyzed fraction. L displays the lysate and the relating lane shows a lot of separate bands. Some of the lysate bands are in a lighter signal strength still present in the first washing fraction (WI). All elution fractions (E1-E11) display the predominant band at $80 \mathrm{kDa}$ but maximum intensity is found in fraction E3. Additionally, to E3, E1 and E5 show bands between $30 \mathrm{kDa}$ and the predominant band at $80 \mathrm{kDa}$. All isolations yielded 10 to $14 \mathrm{mg}$ protein per liter nutrient solution. Concentrations were determined by UV/Vis spectroscopy as described in chapter 3.4.2.

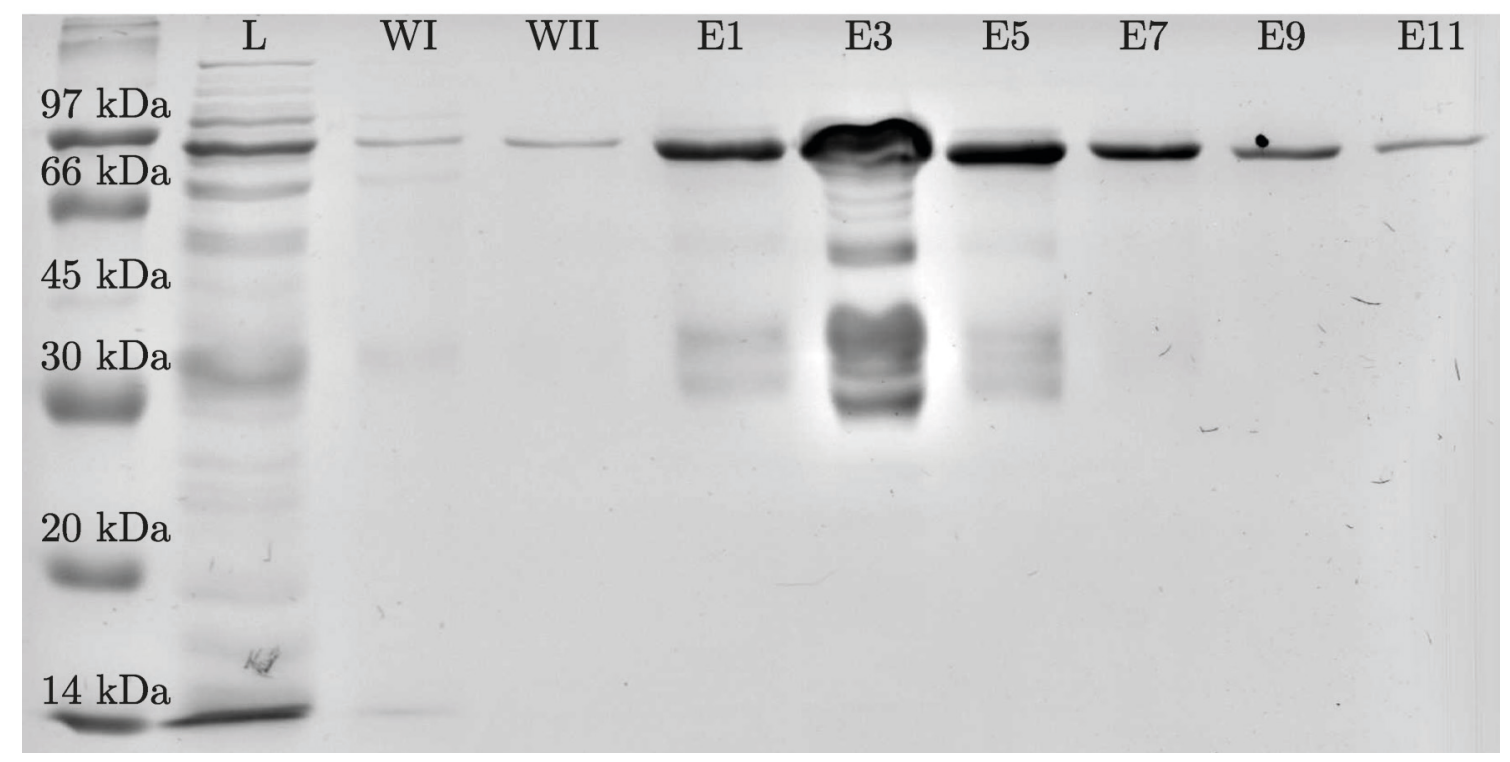

Figure 4.2: Image showing an exemplary SDS-PAGE Gel. A mass standard on the left edge allows relative classification of protein bands. While the lysate (L) is showing a lot of different protein bands, the remaining samples show mostly the predominant band around 80 $\mathrm{kDa}$. The highest band intensity is observed in elution fraction 3 (E3). In these highly concentrated lanes impurities below the predominate band down to $30 \mathrm{kDa}$ are visible. 
Theoretically, ezrin is expected to be visible at $M_{\mathrm{th}}=69.41 \mathrm{kDa}^{[132]}$ but the obtained band with maximum intensity shows a higher mass. This phenomenon is well known in literature and the reason why ezrin was initially termed as 'p81'. ${ }^{[179]}$ A possible and widely accepted explanation can originate from anomalous migration within the SDS-gel due to the protein's highly charged character (213 charged residues $\left.{ }^{[132]}\right) .{ }^{[180]}$ Besides this highly charged character, ezrin harbors a heptaproline sequence inside the linker region which also can be responsible for the anomalous migration of proteins. This sequence imposes conformational restrictions and this is assumed to impact the electrophoretic mobility. ${ }^{[180-182]}$ The further discrepancy of the theoretical expected value and the observations originate from a $N$-terminally fused His 6 -tag which adds a molecular mass of $3.8 \mathrm{kDa}$, including a 24 amino acid long linker.[131] All found impurities were observed before and carry a His6-Tag, which was shown via a Western Blot utilizing an anti-His antibody, ${ }^{[177]}$ and are therefore not eliminated by purification via Ni-NTA agarose chromatography. ${ }^{[116,131,177,178]}$ A purity of $90 \%$ was achieved. Previous experiments have shown that the resulting impurities do not affect the experiments. ${ }^{[116,131]}$

A quite recent study showed ezrin wild type isolation with subsequent removing of the His $_{6}$-tag and additionally anion exchange chromatography and gel filtration to achieve protein in a purity needed to perform small-angle x-ray scattering. [183] To drive the experimental setup closer to physiological conditions an adaption of this protocol for ezrin T567D mutant should be considered.

\subsubsection{Characterization of Ezrin Binding to Solid Supported Lipid Bilayers as a Function of PtdIns $[4,5] \mathrm{P}_{2}$ Content}

- Parts of reflectometric interference spectroscopy measurements have been carried out by Jonas Schäfer as part of his master thesis -

- Experiments in this chapter have been published in "Rheology of MembraneAttached Minimal Actin Cortices" by H. Nöding, M. Schön, C. Reinermann, N. Dörrer, A. Kürschner, B. Geil, I. Mey C. Heussinger, A. Janshoff and C. Steinem, J. Phys. Chem. B, 2018, 122, 4537-4545 [160]-

With the protein ezrin T567D at hand, the binding of the protein to a lipid bilayer doped with PtdIns[4,5] $\mathrm{P}_{2}$ was investigated. Ezrin serves as a linker to establish a connection between the lipid bilayer and an attached minimal actin cortex (MAC) in the model system of this work. Thus, as a first step the surface coverage of ezrin as a function of the PtdIns $[4,5] \mathrm{P}_{2}$ content embedded in the lipid bilayer was characterized. 
Conclusions of a relation between pinning point density for ezrin binding and resulting pinning point density for MACs should be drawn. For the characterization, the spreading of small unilamellar vesicles (SUVs) containing PtdIns $[4,5] \mathrm{P}_{2}$ and subsequent protein binding was monitored by means of reflectometric interference spectroscopy (RIfS), a label-free, non-invasive method (cf. chapter 3.4.1). Utilizing RIfS the resulting adsorption is detected as a change in optical thickness $(\triangle O T)$. A measurement series with different concentrations of PtdIns[4,5] $\mathrm{P}_{2}$ was used to characterize the amount of bound protein, determined as optical thickness. Vesicles and hydrophilic silicon wafers were prepared as described in chapter 3.3.1 and 3.3.2 and subsequent RIfS measurements were performed as described in chapter 3.4.1. Figure 4.3 shows an exemplary RIfS diagram displaying the detected $\triangle O T_{\mathrm{SUV}}$ of spreading POPC/PtdIns[4,5] $\mathrm{P}_{2}$ (92:8) vesicles (B) and subsequently the adsorption of ezrin T567D $\left(\triangle O T_{1}, c=1 \mu \mathrm{M}, \mathrm{D}\right)$. The previous baseline $(\mathrm{A})$ was adjusted by buffer flow with spreading buffer (Na-citrate buffer, $\mathrm{A} / \mathrm{A}_{1}$ ) as well as the plateau with a flow of protein buffer (E1 buffer, C). After successful protein adsorption of ezrin T567D (D) further rinsing with protein buffer (E) led to a decrease in optical thickness, which is the result of desorption of reversibly bound protein. The resulting difference between the plateau after protein desorption (E) and optical thickness after SUV spreading is termed as $\Delta\left(\Delta O T_{2}\right)$ for irreversible ezrin T567D binding, while $\Delta\left(\Delta O T_{1}\right)$ identifies reversible ezrin binding (Figure $4.3 \mathrm{D}$ ). The plateaus as well as the baseline were fitted using $\Delta O T=$ const. to determine exact $\Delta(\triangle O T)$-values. PtdIns $[4,5] \mathrm{P}_{2}$ concentrations of the vesicles were altered in a range of 1-8 mol \%.

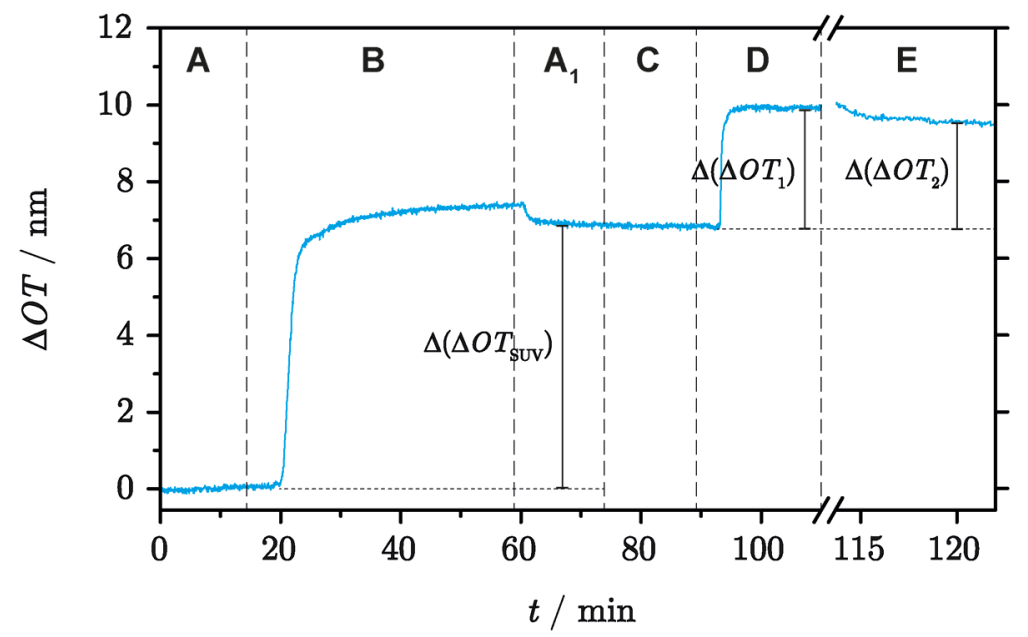

Figure 4.3: Exemplary RIfS diagram showing ezrin T567D binding to a POPC/PtdIns $[4,5] P_{2}$ (92:8) lipid bilayer. A: A baseline was established by rinsing the system with spreading buffer. B: Subsequent spreading of SUVs (POPC/PtdIns[4,5]P 2 (92:8)) led to an increase of around $7 \mathrm{~nm}$ in optical thickness $(O T)$, which adjusts after rinsing with Na-citrate buffer $\left(\mathbf{A}_{\mathbf{1}}\right)$ to $6.8 \mathrm{~nm}$. C-D: The system was transferred into protein buffer $(\mathbf{C})$ and ezrin T567D $(c=1 \mu \mathrm{M})$ was injected in the system which led to a further increase in $\triangle O T$ 
(D). E: After rinsing again with protein buffer a small decrease was observed. Changes in $O T \mathrm{~s}$ were determined in this example: $\Delta\left(\Delta O T_{1}\right)=3.1 \mathrm{~nm}$ and $\Delta\left(\Delta O T_{2}\right)=2.7 \mathrm{~nm}$.

Spreading of POPC/PtdIns[4,5] $\mathrm{P}_{2}$ SUVs in molar ratios from $1 \mathrm{~mol} \% \operatorname{PtdIns}[4,5] \mathrm{P}_{2}$ up to $8 \mathrm{~mol} \%$ resulted in $\Delta O T_{\mathrm{SUV}}$ of $(6.8 \pm 0.6) \mathrm{nm}(N=34)$. With equation 3.4 , the physical thickness was calculated using the refractive index. To be more precise, both refractive indices of POPC and PtdIns $[4,5] \mathrm{P}_{2}\left(n_{\mathrm{POPC}}=1.47, n_{\mathrm{PtdIns}[4,5] \mathrm{P} 2}=1.54\right)^{[184,185]}$ were taken into account, yielding a refractive index for the complete lipid bilayer $\left(n_{\mathrm{LB}}\right)$. $\chi$ denotes the respective molar fractions of POPC and PtdIns[4,5] $\mathrm{P}_{2}$ (equation 4.1):

$$
n_{L B}=n_{P O P C} \cdot \chi_{P O P C}+n_{P t d I n s[4,5] P_{2}} \cdot \chi_{P t d I n s[4,5] P_{2}}
$$

Results of $\Delta O T_{\mathrm{SUV}}$ and physical thickness $d$ according to the molar ratio of PtdIns $[4,5] \mathrm{P}_{2}$ (in $N$ experiments) are summarized in Table 4.1.

Table 4.1: Refractive indices and the resulting physical thicknesses $d$ of spread lipid bilayers.

\begin{tabular}{|c|c|c|c|c|c|c|c|}
\hline$\chi_{P t d I n s[4,5] P_{2}}$ & $1 \underset{\%}{1 \mathrm{~mol}}$ & $2 \mathrm{~mol} \%$ & $3 \mathrm{~mol} \mathrm{\%}$ & $4 \mathrm{~mol} \mathrm{\%}$ & $5 \mathrm{~mol} \%$ & $6 \mathrm{~mol} \mathrm{\%}$ & $8 \mathrm{~mol} \mathrm{\%}$ \\
\hline$n_{\mathrm{LB}}$ & 1.471 & 1.471 & 1.472 & 1.472 & 1.473 & 1.473 & 1.474 \\
\hline$d / \mathbf{n m}$ & $4.4 \pm 0.2$ & $4.8 \pm 0.3$ & $4.3 \pm 0.3$ & $4.3 \pm 0.2$ & $5.3 \pm 0.3$ & $4.6 \pm 0.1$ & $4.4 \pm 0.2$ \\
\hline$N$ & 4 & 5 & 5 & 4 & 6 & 3 & 7 \\
\hline
\end{tabular}

As there was no dependence of the lipid bilayer's physical thickness on the PtdIns $[4,5] \mathrm{P}_{2}$ content found, a total physical thickness averaged over all measurements and PtdIns $[4,5] \mathrm{P}_{2}$ ratios was determined to $(4.6 \pm 0.4) \mathrm{nm}$. This value corresponds well with those reported with literature, as typical phospholipid bilayers show a physical height of 4-5 nm.[186] Furthermore, previous studies using RIfS showed an almost identical lipid bilayer height of $4.5 \mathrm{~nm}$ spreading DOPC and 1,2-dioleoyl-sn-glycero-3phosphoethanolamine-N-[4-(p-maleimidomethyl)-cyclohexanecarboxamide] (MCCDOPE) in a ratio of 9:1.[130] In contrast to that, other studies determined the physical thickness of a pure POPC bilayer at $20{ }^{\circ} \mathrm{C}^{[187]}$ of $(3.98 \pm 0.08) \mathrm{nm}$, which is slightly lower than the measured value. However, the compared literature value was determined by small-angle neutron and x-ray scattering (SAXS) and this technique requires different experimental conditions and detects electron densities. Both facts could be reasons to explain a slightly lower detected physical thickness as both techniques are hard to compare. Furthermore, in this setup, no pure POPC bilayer was measured. 
The added PtdIns[4,5] $\mathrm{P}_{2}$ lipids contribute a larger head group compared to POPC.[59] Therefore, the physical thickness should be slightly increased by adding subsequently PtdIns $[4,5] \mathrm{P}_{2}$. One could argue that the physical thickness should increase with increasing molar ratio of PtdIns $[4,5] \mathrm{P}_{2}$ but since this is not the case, the additional head group height seems to vanish into the signal-to-noise ratio. Considering further experiments in literature, the physical thickness of a lipid bilayer consisting of DPPC/PtdIns [4,5] $\mathrm{P}_{2}$ (DPPC = 1,2-dipalmitoyl-sn-glycero-3-phosphocholine, 97:3) was determined to be around $6.0 \mathrm{~nm}$ by means of AFM. ${ }^{[56]}$ In conclusion, the detected value is in good accordance with the literature. The value is almost identical to measured values with the same technique and it is therefore assumed that the lipid bilayer is covering the whole surface.

Subsequently to the formation of the lipid bilayer, ezrin T567D was added and the change of $O T$ was monitored (cf. Figure $4.3 \mathrm{D}$ ). To check for unspecific binding of ezrin T567D to POPC, two measurements in absence of PtdIns[4,5] $\mathrm{P}_{2}$ were performed which resulted in no change in optical thickness. Molar ratios of $\operatorname{Ptd} \operatorname{Ins}[4,5] \mathrm{P}_{2}$ were then altered in a range of 0-8 mol \%. As Figure 4.4 displays, increasing the molar ratios of PtdIns $[4,5] \mathrm{P}_{2}$ results in a larger $\Delta O T$. A higher $\Delta\left(\Delta O T_{2}\right)$ is caused by a larger amount of irreversibly adsorbed ezrin T567D.

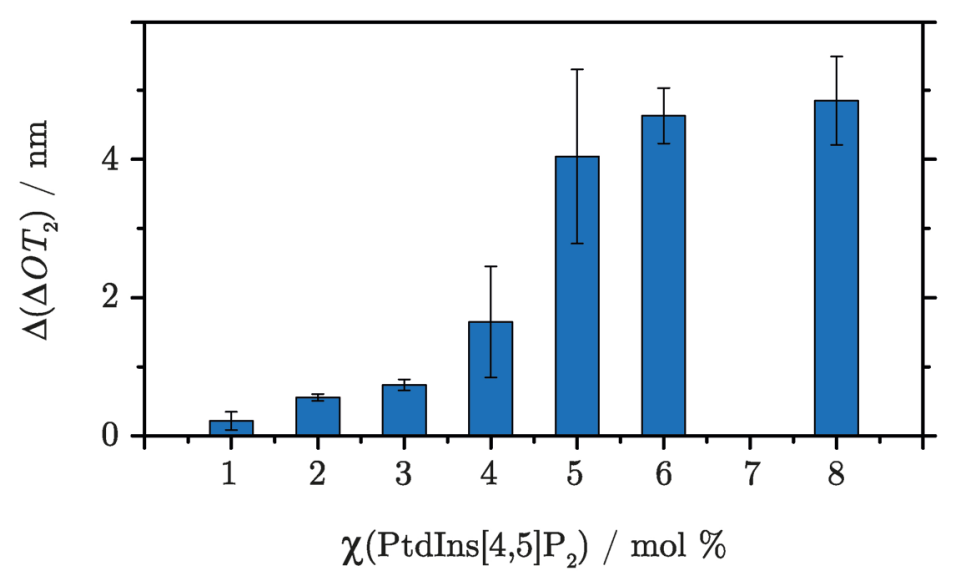

Figure 4.4: Representation of increasing optical thickness $O T$ caused by ezrin T567D adsorption depending on PtdIns $[4,5] \mathbf{P}_{2}$ content. Optical thickness of irreversible ezrin T567D binding $\Delta\left(\Delta O T_{1}\right)$ increases sigmoidal up to $(4.7 \pm 0.6) \mathrm{nm}$ at $8 \mathrm{~mol} \%$ PtdIns $[4,5] \mathrm{P}_{2}(N=7)$. While at low PtdIns $[4,5] \mathrm{P}_{2}$ concentrations only a slight increase from $(0.08 \pm 0.13) \mathrm{nm}\left(1 \mathrm{~mol} \% \operatorname{PtdIns}[4,5] \mathrm{P}_{2}, N=5\right)$ via $(0.4 \pm 0.1) \mathrm{nm}\left(2 \mathrm{~mol} \% \operatorname{PtdIns}[4,5] \mathrm{P}_{2}\right.$, $N=5)$ to $(0.6 \pm 0.1) \mathrm{nm}\left(3 \mathrm{~mol} \% \operatorname{PtdIns}[4,5] \mathrm{P}_{2}, N=5\right)$ was observed, the increase up to $(3.9 \pm 1.3) \mathrm{nm}\left(5 \mathrm{~mol} \% \operatorname{PtdIns}[4,5] \mathrm{P}_{2}, N=3\right)$ via $(1.5 \pm 0.13) \mathrm{nm}\left(4 \mathrm{~mol} \% \operatorname{PtdIns}[4,5] \mathrm{P}_{2}\right.$, $N=4)$ is more rapid. From $6 \mathrm{~mol} \%$ with an $\Delta\left(\Delta O T_{1}\right)$ of $(4.5 \pm 0.4) \mathrm{nm}(N=3)$ no significant increase to $8 \mathrm{~mol} \% \operatorname{PtdIns}[4,5] \mathrm{P}_{2}$ was detected. Error bars display the standard deviation of the mean value. 
Starting with a ratio of $1 \mathrm{~mol} \% \operatorname{PtdIns}[4,5] \mathrm{P}_{2}$ a change in optical thickness $\Delta\left(\Delta O T_{2}\right)=(0.08 \pm 0.13) \mathrm{nm}$ is observed. The adsorbed protein ratio increases slightly till a molar content of $4 \mathrm{~mol} \%$ PtdIns $[4,5] \mathrm{P}_{2}$ shows a sharp signal intensity rise to $\Delta\left(\Delta O T_{2}\right)=(1.5 \pm 0.8) \mathrm{nm} \quad$ in contrast to $\Delta\left(\Delta O T_{2}\right)=(0.6 \pm 0.1) \mathrm{nm} \quad(3 \mathrm{~mol} \%$ $\left.\operatorname{PtdIns}[4,5] \mathrm{P}_{2}\right)$. This increase gets even more rapid up to $\Delta\left(\Delta O T_{2}\right)=(3.9 \pm 1.3) \mathrm{nm}$ when the receptor lipid content is increased up to $5 \mathrm{~mol} \%$. After a certain ratio of 6 mol \% PtdIns $[4,5] \mathrm{P}_{2}$ no further increase in change of optical thickness of ezrin T567D binding was observed (Figure 4.4, compare $\chi_{\text {PtdIns }[4,5] P_{2}}=6 \mathrm{~mol} \%$ and $\chi_{\text {PtdIns }[4,5] P_{2}}=$ $8 \mathrm{~mol} \%)$ after reaching $\Delta\left(\Delta O T_{2}\right)=(4.5 \pm 0.4) \mathrm{nm}$. Even measurements on $8 \mathrm{~mol} \%$ $\operatorname{PtdIns}[4,5] \mathrm{P}_{2}$ showed no significant increase in optical thickness $\Delta\left(\Delta O T_{2}\right)$ anymore. Throughout the entire series of experiments, a sigmoidal-like increase is observable. No further increase and staying at the same level of optical thickness indicated that the surface coverage of protein on the lipid bilayer is already at its maximum. Therefore, this limit is set to $100 \%$ of ezrin T567D surface coverage.

Furthermore, a direct correlation between optical thickness and physical thickness is given using the refractive index (equation 3.4) as described in chapter 3.4.1. In previous studies the refractive index for the protein layer is given as $n=1.5,[188,189]$ staying close to the refractive index of silicon dioxide and in the range of 1.36 to 1.55 as evaluated by Vörös et al. ${ }^{[127]}$ In this work the refractive index of an adsorbed protein layer $\left(n_{\mathrm{P}}\right)$ is presumed with the mean value $\left(n_{\mathrm{P}}=1.455\right)$ of this range as it was already done in a relating study working with ezrin T567D.[56] The physical thicknesses of the respective protein layers were evaluated and displayed in Figure 4.5.

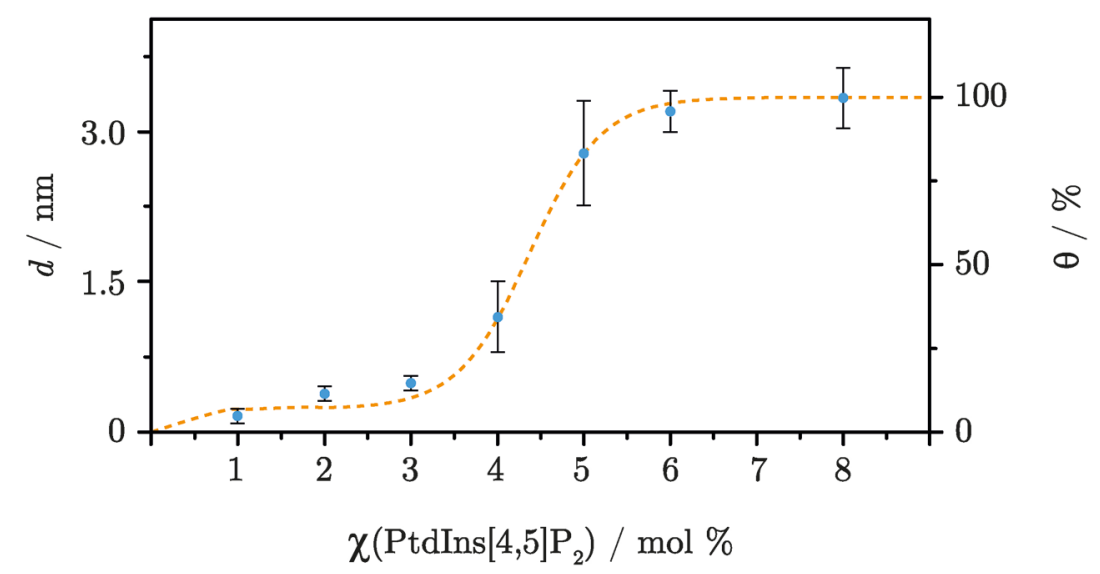

Figure 4.5: Physical thickness and relating relative surface coverage of ezrin T567D $(\theta)$ as a function of PtdIns[4,5] $\mathbf{P}_{2}$ content. Physical thicknesses were calculated using equation 3.4 and the respective $O T$ values of Figure 4.4 determined by means of RIfS. The sigmoidal-like increase in protein coverage is elucidated in orange.

To compare the height to literature values, an atomic force micrograph of a DPPC/PtdIns [4,5] $\mathrm{P}_{2}$ (97:3) lipid bilayer incubated with ezrin T567D is taken. ${ }^{[56]}$ This 
AFM image (cf. Figure 4.6) was used to determine the protein's height. To compare the surface coverage, in this case $3 \mathrm{~mol} \% \operatorname{PtdIns}[4,5] \mathrm{P}_{2}$, a simple threshold analysis was implemented (cf. Figure 4.6). This threshold analysis revealed a protein coverage of around $25 \%$ which is slightly above the detected coverage via RIfS (Figure 4.5) at $3 \mathrm{~mol} \% \operatorname{Ptd} \operatorname{Ins}[4,5] \mathrm{P}_{2}$. The detected value of protein surface coverage fits quite good into the resulting arrangement of ezrin T567D coverage which supports the assumption of full surface coverage at $6 \mathrm{~mol} \% \operatorname{PtdIns}[4,5] \mathrm{P}_{2}$ with a physical protein layer thickness of around $3.2 \mathrm{~nm}$.
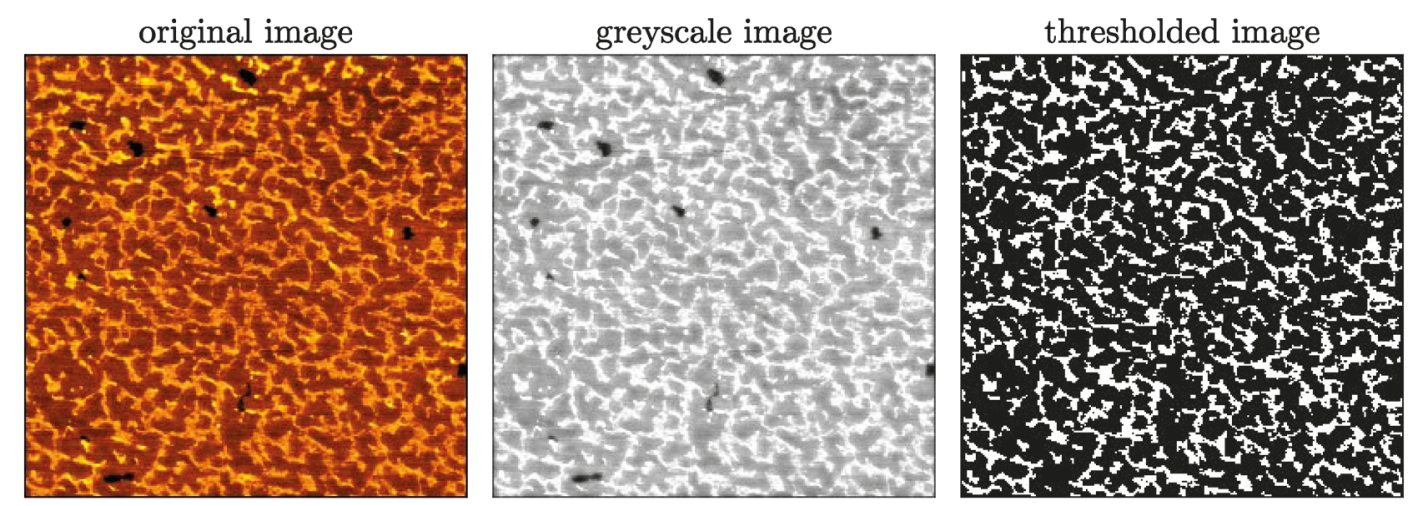

Figure 4.6: Threshold analysis of an atomic force micrograph obtained by Shabardina et $\boldsymbol{a l}^{.{ }^{[56]}}$ The literature obtained AFM micrograph displays ezrin T567D (light brown) incubated on a lipid bilayer consisting of DPPC/PtdIns $[4,5] \mathrm{P}_{2}$ (97:3, dark brown). Besides different matrix lipids the experimental conditions are nearly the same as ezrin T567D is bound to $\mathrm{Ptd} \operatorname{Ins}[4,5] \mathrm{P}_{2}$. An applied threshold separates pixels relating to protein coverage (white area of thresholded image) were compared to the maximal amount of image pixels resulting in a coverage of around $25 \%$. Original image retrieved from Shabardina et al. and reprinted with permission from Elsevier. ${ }^{[56]}$

Defects in the solid supported lipid bilayer (black areas in Figure 4.6, original image) were not taken into consideration since RIfS is also averaging an area of around $1 \mathrm{~mm}^{2}$. The comparison of both analysis is therefore consisting in ignoring membrane defects and contribute to the error of the measurement. AFM and RIfS measurements therefore agree with each other concerning the surface coverage but do not explain the fairly low total height of the protein layer with respect to recent literature. Phang et al. used small-angle x-ray scattering data of ezrin domains and the crystal structure of the full length sfmoesin ${ }^{[190]}$ (moesin of the insect Spodoptera frugiperda) to develop a model of the monomeric closed conformation (dormant) revealing a height of $(16.5 \pm 0.5) \mathrm{nm}$ in case of the maximum chord of the extended coiled coil.[183] As the crystal structure of full-length ezrin is still unclear the crystal structure of $N$-ERMAD serves as a benchmark (Figure 4.7) for further considerations. 


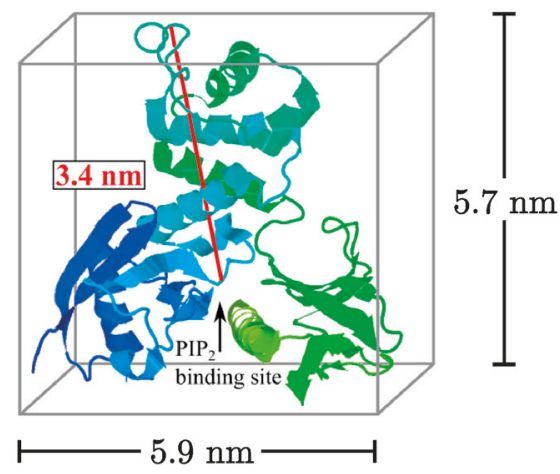

Figure 4.7: Crystallographic structure of $N$-ERMAD showing three subdomains (F1, F2 and F3). ${ }^{[56]}$ Structure is shown in the possible orientation of PtdIns $[4,5] \mathrm{P}_{2}$-binding (abbreviated as $\mathrm{PIP}_{2}$ inside the illustration). Crystallographic dimensions demonstrate a domain height of $3.4 \mathrm{~nm}$ in case of bound protein. Retrieved from Shabardina et al. and reprinted with permission from Elsevier ${ }^{[56]}$

Comparing the detected protein layer's physical thickness at full surface coverage of $3.2 \mathrm{~nm}$ with the estimated height of $N$-ERMAD (cf. Figure 4.7) the obtained value is still below the assumed height of solely the $N$-terminal domain. This phenomenon of too low protein layers in case of ezrin T567D adsorbed to PtdIns $[4,5] \mathrm{P}_{2}$ is known in literature as an earlier study detected a significantly lower height of ezrin T567D mutant by means of AFM of about $(1.6 \pm 0.3) \mathrm{nm}$. Interestingly, while RIfS averages an area of about $1 \mathrm{~mm}^{2}$, AFM can detect single protein structures. Based on these findings, Shabardina et al. assumed a binding model where the activated mutant of ezrin is more loosely packed when it is bound to artificial bilayers doped with PtdIns[4,5] $\mathrm{P}_{2}$ (cf. Figure 4.8 B)..56] 

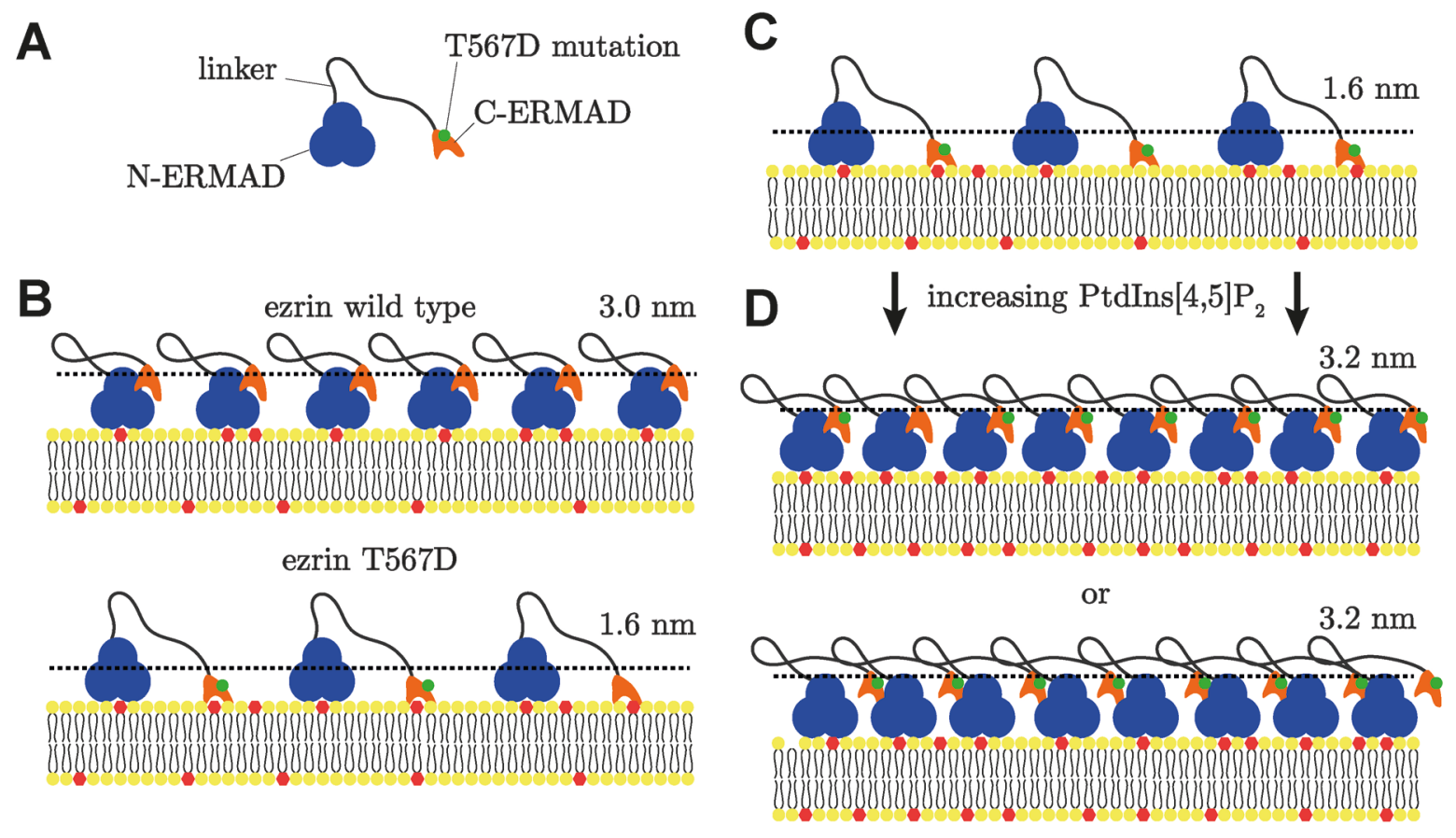

Figure 4.8: Proposed model of ezrin T567D's arrangement on a lipid bilayer doped with PtdIns $[4,5] \mathbf{P}_{2}$. A: Schematic key how ezrin T567D is illustrated within the graphic. B: Model adapted from Shabardina et al. ${ }^{[56]}$ It is assumed that ezrin T567D is loosely packed on a solid supported lipid bilayer (SLB) in contrast to the wild type. C-D: Developing this model, with increasing ratio of PtdIns[4,5]P2 the unspecific binding of the $C$-ERMAD on the SLB could be displaced by the high surface coverage. Either intramolecular (D top) or intermolecular (D bottom) interactions of ezrin T567D molecules are assumed to be the reason why the average height is only getting close to the structural height of $N$-ERMAD and not exceed it.

The model which was proposed for the situation on $3 \mathrm{~mol} \% \operatorname{PtdIns}[4,5] \mathrm{P}_{2}$ suggests that the $C$-ERMAD takes a binding place at the lipid bilayer. This means that the average height over the whole square-micrometer detected by RIfS is significantly lower (cf. Figure $4.8 \mathrm{C}$ ). An increase in pinning points would then lead to a more densely packed protein layer displacing the $C$-ERMAD from the pinning points within the SLB (cf. Figure $4.8 \mathrm{D}$ ). The largest detected average height of the protein monolayer is still smaller than the height determined for $N$-ERMAD crystal structure. If the $C$-ERMAD of ezrin would protrude from the protein layer, thus a higher average height should be obtained. Furthermore, the $C$-ERMAD could take space between $N$-ERMADs within the protein monolayer, but without any molecular interaction. It seems very unlikely that binding of $N$-ERMAD to PtdIns $[4,5] \mathrm{P}_{2}$ cannot displace the $C$-ERMAD, therefore interactions between the protein domains should be taken into consideration. It was shown that ezrin T567D still forms $N$-ERMAD- $C$-ERMAD contacts, inter- as well as intramolecular, although it carries an additional charge. ${ }^{[54]}$ Consequently, a further study revealed that even ezrin mutants form a closed conformation where $C$-ERMAD assembles at the $N$-ERMAD.[191] The activated $C$-ERMAD therefore seems to need a 
binding partner where this model proposes different affinities. It is able to bind a free binding place at the lipid bilayer as well as a $N$-ERMAD domain.

Therefore, in a densely packed ezrin monolayer an intermolecular effect is also possible as the $C$-ERMAD can assemble at the $N$-ERMAD even of the T567D mutant (illustrated in Figure 4.8 D, bottom). ${ }^{[54,183,191]}$ This cooperative effect in ezrin binding was already reported for ezrin wild type and therefore contingent for the T567D mutant as C- and $N$-ERMAD assembling was reported for both proteins. ${ }^{[106]}$ Recapitulated, the employed experiments in this chapter support and develop the model presented by Shabardina et al. as the maximum detected height does not increase further than $(3.2 \pm 0.4) \mathrm{nm}$ which corresponds to the height of $N$-ERMAD (Figure 4.7$)$.

\subsubsection{Fluorescent Labeling of Ezrin T567D}

- Experiments have been carried out by Malte Holmer as part of his bachelor thesis -

To characterize ezrin's distribution on different substrates, the protein was visualized by labeling the protein with the fluorophore Atto 488. Atto 488 was bound to a cysteine residue via a Michael addition as described in chapter 3.2.7. UV/Vis spectroscopy was used to determine the concentration of protein and degree of labeling (DOL). Unlike described in equation 3.13 protein concentration can only be calculated by taking a correction factor into account (equation 3.14) respecting the intensity of the fluorophore at the measured wavelength. In Figure 4.9 an exemplary UV/Vis spectrum of a concentrated elution fraction is shown in blue.

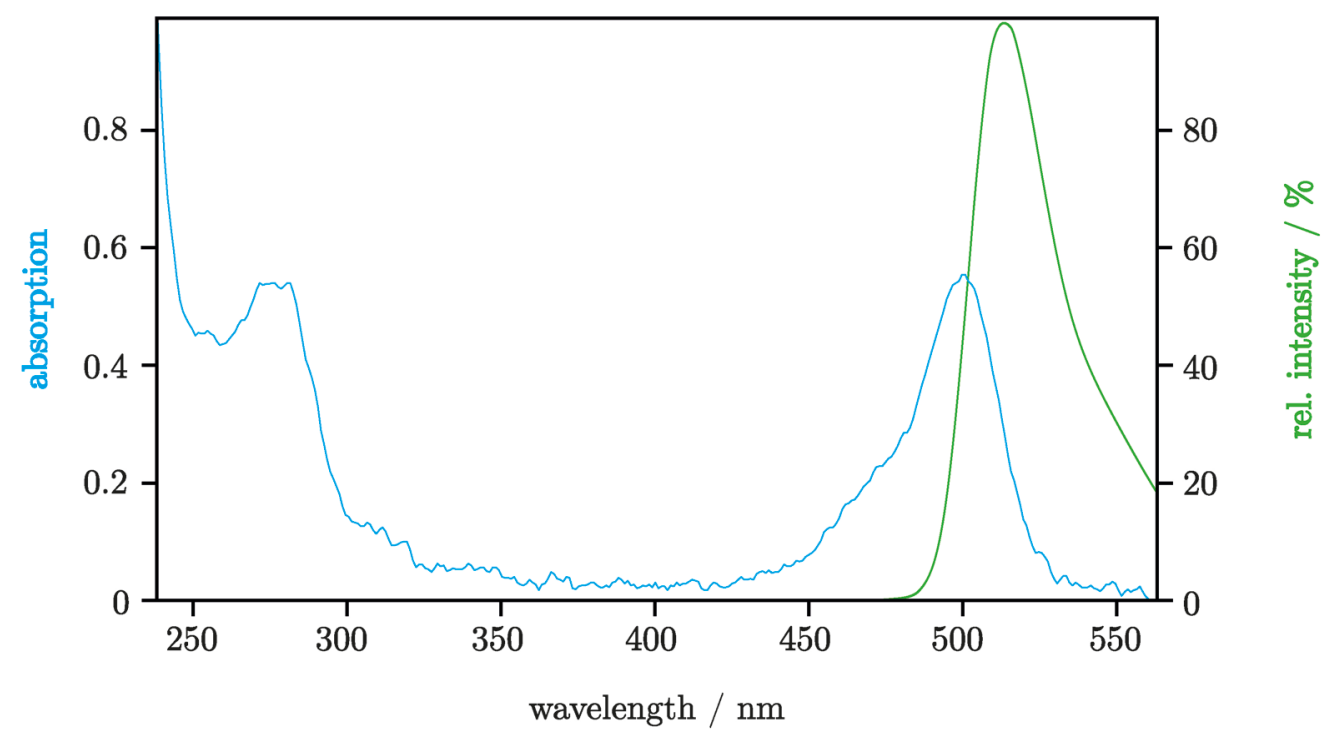

Figure 4.9: Exemplary UV/Vis spectrum of an elution fraction as outcome of a labeling reaction. In blue an absorption spectrum of a typical elution fraction is shown. In comparison with the green intensity spectrum of free fluorophore Atto 488 a clear correlation 
between the second absorption peak at $502 \mathrm{~nm}$ and the emission of Atto 488 is detected. The first maximum at $280 \mathrm{~nm}$ derives therefore characteristically from the protein.

The spectrum shows two maxima at $280 \mathrm{~nm}$ and $502 \mathrm{~nm}$. As described in chapter 3.2.7, the absorption peak at $280 \mathrm{~nm}$ is characteristic for proteins due to tryptophan, phenylalanine and tyrosine residues. The absorption spectrum of the Atto 488 fluorophore (Figure 4.9, green) indicates that the second maximum derives from the bound fluorophore.[133] For all fluorescence labeling reactions a yield of (30 \pm 13$) \%$ with a DOL of $0.7 \pm 0.2$ was achieved $(N=5)$. Noticeable there are two cysteine residues found in the amino acid sequence of ezrin (Figure 4.1) which would theoretically allow a DOL of 2 as $100 \%$ success rate of labeling proteins. Previous studies in our group showed that labeling of ezrin wild type and ezrin T567D with Alexa Fluor ${ }^{\circledR}$ 633-maleimide was performed successfully with DOL of 1.4 for the wild type protein and 2.0 for ezrin T567D.[131] On the other hand, Blin et al. described a labelling procedure where they add a further cysteine residue at the $C$-terminus as they explained both cysteine residues would be inaccessible.[192] Concerning the crystal structure, the two cysteine residues were both detected in the $N$-ERMAD, C117 is located in the F2 subdomain and C284 in the F3 subdomain.[193] With the crystal structure of $N$-ERMAD in hand (cf. Figure 3.14), it was demonstrated that C284 is located inside an $\alpha$-helix within the domain while $\mathrm{C} 117$ is located in a so called [IL]-x-C-X-x-[DE] motif. This structure is a target motif for cysteine S-nitrosylation mediated by a transnitrosylase complex belonging to the glyceraldehyde-3-phosphate dehydrogenase family.[132,194] Therefore, C117 is not only a central amino acid in an active center, it should also be ensured that the cysteine residue is accessible for nitrosylation. As the fluorophore is way smaller than an enzyme complex it seems reasonable that $\mathrm{C} 117$ is easier accessible than $\mathrm{C} 284$ which is located in an $\alpha$-helix. More vigorous reaction conditions could make the second cysteine accessible but considering that only one, easy accessible cysteine is labeled, this is still enough to make the protein visible by means of fluorescence microscopy. Consequently, a DOL of 1 means that every protein carries the marker. The yield achieved in these experiments corresponding to the described assumptions is therefore $(70 \pm 13) \%$ success rates in labeling ezrin T567D which is quite typical for cysteine labeling reactions. [110] 


\subsection{Self-organization of F-actin Networks depending on the Pinning Point Density}

- Experiments in this chapter have been published in "Rheology of MembraneAttached Minimal Actin Cortices" by H. Nöding, M. Schön, C. Reinermann, N. Dörrer, A. Kürschner, B. Geil, I. Mey, C. Heussinger, A. Janshoff and C. Steinem, J. Phys. Chem. B, 2018, 122, 4537-4545 [160] -

In cells, ezrin is known to link the plasma membrane (via PtdIns[4,5] $\mathrm{P}_{2}$ lipids) to filamentous actin (F-actin) networks. ${ }^{[50]}$ It was shown that the pseudo-phosphorylated mutant ezrin T567D is able to establish this connection similarly in in vitro experiments. ${ }^{[51,116]}$ Thus, F-actin networks will be attached to ezrin T567D decorated lipid bilayers containing various ratios of PtdIns $[4,5] \mathrm{P}_{2}$, which describe the pinning

point density. The content of the receptor lipid PtdIns[4,5] $\mathrm{P}_{2}$ and ezrin T567D binding was correlated in chapter 4.1.3 and this correlation will be used in the following section, in order to characterize the self-organization of these F-actin network in dependence of the pinning point density. First, F-actin network gels were produced by polymerization from globular protein (G-actin) solutions. These network gels were referred to as pre-polymerized F-actin gels, because the polymerization was completed before any further investigations were pursued. Furthermore, filaments were stabilized and labeled using Alexa Fluor ${ }^{\circledR}$ 488-phalloidin.

\subsubsection{Characterization of 3D pre-polymerized F-actin gels}

F-actin networks were polymerized as described in chapter 3.3 .5 resulting in three-dimensional (3D) network gels. In order to investigate these pre-polymerized 3D F-actin networks, they were filled either in a microfluidic channel or a microfluidic chamber (cf. Figure 3.5). Utilizing these microfluidic chambers or channels, F-actin networks could be monitored by means of confocal laser scanning microscopy. Resulting F-actin gels were found in many different morphologies, as shown in Figure 4.10. All images are exemplary representations of network morphologies. In general, F-actin networks within a microfluidic channel showed a different visual appearance demonstrating a clear orientation, in contrast to networks monitored in microfluidic chambers showing no preferred orientation. 

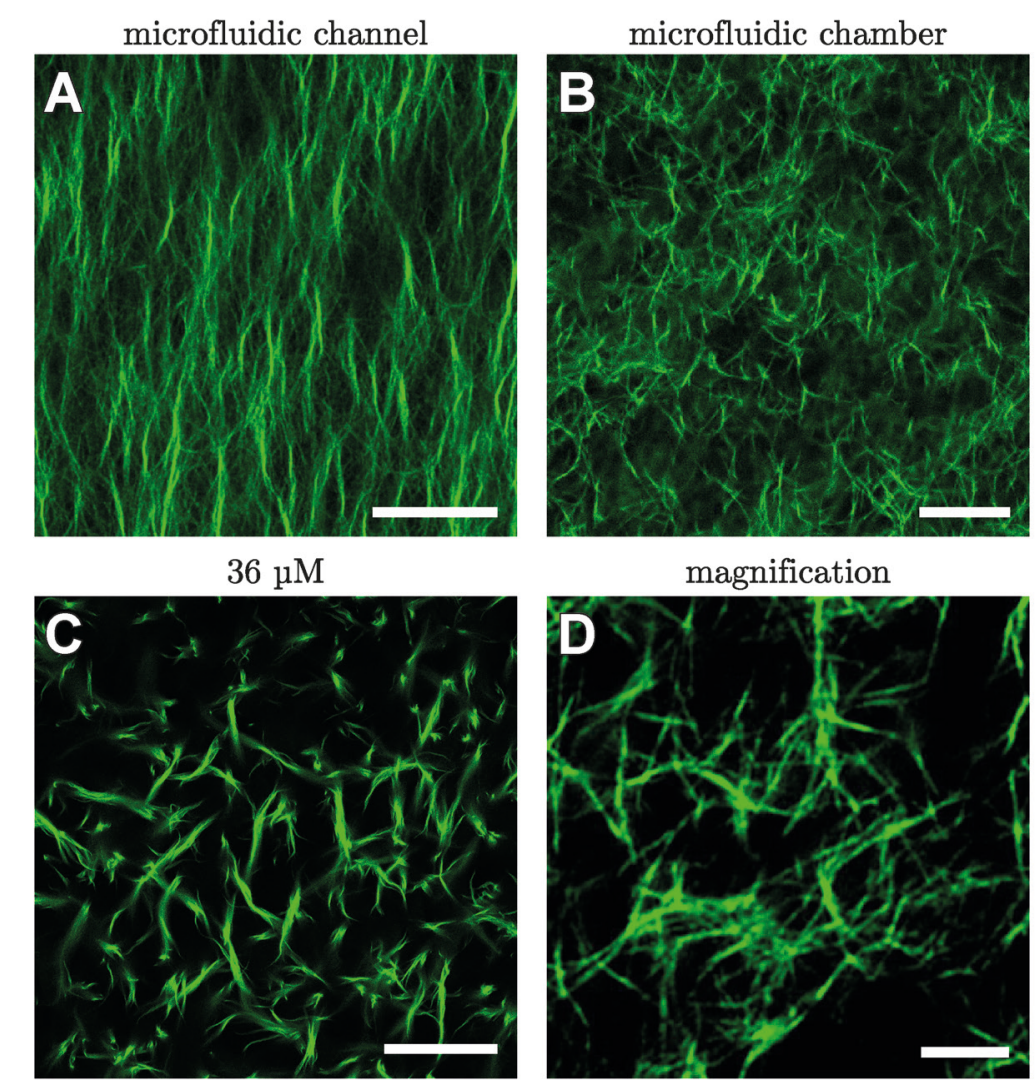

Figure 4.10: Fluorescence micrographs reveal different morphologies of 3D F-actin gels. A: F-actin labeled with Alexa Fluor ${ }^{\circledR}$ 488-phalloidin was polymerized at a concentration of $8 \mathrm{\mu M}$ and imaged in a microfluidic channel. In case of microfluidic channels, actin filaments show a preferred orientation along the channel direction. B: A similar network $(8 \mu \mathrm{M})$ was imaged in a microfluidic chamber where no preferred orientation was observed. C: F-actin, polymerized at a higher concentration of $36 \mu \mathrm{M}$, appears to be less branched and shows thicker filamentous structures. D: A magnification of a network morphology like the network shown in (B) reveals different fluorescence intensities for filamentous structures. Scale bars: A-B: $20 \mu \mathrm{m}$; C: $50 \mu \mathrm{m}$; D: $5 \mu \mathrm{m}$.

It has been shown that structural differences within the F-actin networks can be observed, depending on the usage of a microfluidic chamber or a microfluidic channel. In channels, actin filaments often show a preferred orientation along the channel direction (Figure $4.10 \mathrm{~A}$ ), whereas images of networks inside a microfluidic chamber reveal a highly branched network morphology (Figure $4.10 \mathrm{~B}$ ) without an apparent orientation. A magnification of these networks shows that filamentous structures vary in fluorescence intensity, suggesting different thicknesses which can be a result of the presence of bundled single filaments (Figure 4.10 D). When F-actin was polymerized from a G-actin solution of $36 \mu \mathrm{M}$, in contrast to the described polymerization in chapter 3.3.5 using a lower G-actin concentration $(c=8 \mu \mathrm{M})$, a more bundled network, as shown in Figure $4.10 \mathrm{C}$, can be observed. The resulting structures appear to be significantly thicker than filaments of Figure $4.10 \mathrm{~A}$ and B. These images present 
mentioned trends which were observed generally in all experiments, although each network constitution is strongly heterogeneous.

Furthermore, the total extent of an F-actin network inside a microfluidic chamber was investigated. Many fluorescence micrographs, imaged by CLSM, were combined to achieve a complete $z$-stack of an Alexa Fluor ${ }^{\circledR}$ 488-labeled 3D F-actin gel. One exemplarily characterized 3D F-actin network gel is shown in Figure 4.11, which is presenting single recorded $z$-planes (cf. Figure $4.11 \mathrm{~A} / \mathrm{C}$ ), the combination of many slices as a z-projection averaged over many layers (cf. Figure $4.11 \mathrm{D}$ ) as well as an orthogonal view of the layers (cf. Figure $4.11 \mathrm{~B}$ ).
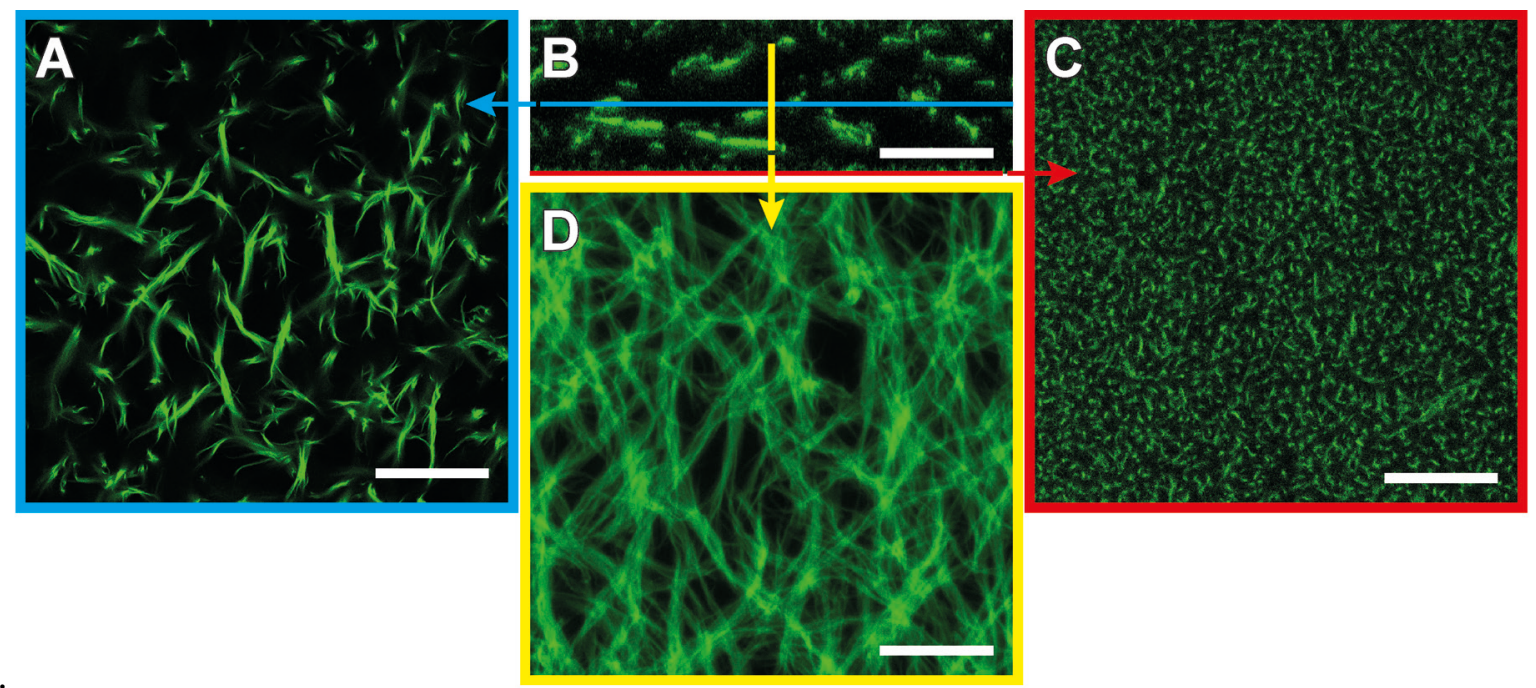

Figure 4.11: Fluorescence micrograph of a 3D F-actin gel's extension in a microfluidic chamber. A (cyan): A typical confocal image of a plane at medium height of the microfluidic chamber (see arrow in $(\mathbf{B})$ ). B: Cross section ( $x$-z-direction) of a $z$-stack through a microfluidic chamber. On top and bottom plane, a layer of fluorescence intensity is observed (C) and the filamentous network extents between these planes (A/C). C (red): Confocal image recorded at the bottom plane of the microfluidic channel. Short fragments of fluorescence intensity can be observed. D (yellow): A projection of fluorescence micrographs of the labeled F-actin network in axial direction is shown, indicated by the yellow line in (B). Filamentous structures appear larger in contrast to single recorded layers as shown in (A). Scale bars: $20 \mu \mathrm{m}$. Total height of the $z$-stack (B): $106 \mu \mathrm{m}$.

Interestingly, the microscope slide and the coverslip are clearly detectable, because the observed fluorescence signal at top and bottom plane differ from the one obtained between these two slides (compare Figure 4.11 A and Figure 4.11 C). Furthermore, the fluorescence in the middle of the microfluidic chamber showed clear filamentous structures (cf. Figure $4.11 \mathrm{~A}$ ), while at the detected top or bottom plane (cf. Figure $4.11 \mathrm{C}$ ) only short fragments of fluorescence intensity can be observed. This indicates that either filament ends attaching to the coverslip or short filament fragments are observed at these planes. In between these two layers assigned to top and bottom of the chamber, the extension of a filamentous network is observed. Single confocal images 
of one slice of the microfluidic chamber shows the typical filamentous morphology as already presented in Figure 4.10. A projection of many confocal slices into one layer, like Figure $4.11 \mathrm{~A}$, by averaging the bleaching corrected fluorescence intensity reveals the total propagation of the obtained filaments, or filament bundles, through the whole microfluidic chamber (Figure 4.11 D). The length of the structures appears to be much longer in the z-projection than in a single confocal image, because many filamentous structures pass through various $z$-planes.

To analyze the 3D images of the obtain filaments, in order to determine the filament lengths, the software SOAX (cf. chapter 3.6.1.2) was used. In chapter 3.6.1 the two methods of artificial retina and the software SOAX are presented, which can skeletonize filamentous networks from fluorescence micrographs. Using these skeletons, quantitative parameters, like the filament length, can be obtained. While the artificial retina is a method based on the image enhancement of $2 \mathrm{D}$ images, SOAX is a software precisely developed for "quantification of 3D biopolymer networks".[162] Therefore, SOAX is well suited for analyzing the obtained 3D stacks of fluorescence micrographs, because in case of using the artificial retina obtained information of the filament length extension in z-direction would be lost. An exemplary image stack of a 3D F-actin gel which was evaluated using SOAX is shown in Figure $4.12 \mathrm{~A} / \mathrm{B}$. The detected filament lengths as issued from SOAX are shown in Figure 4.12 C.
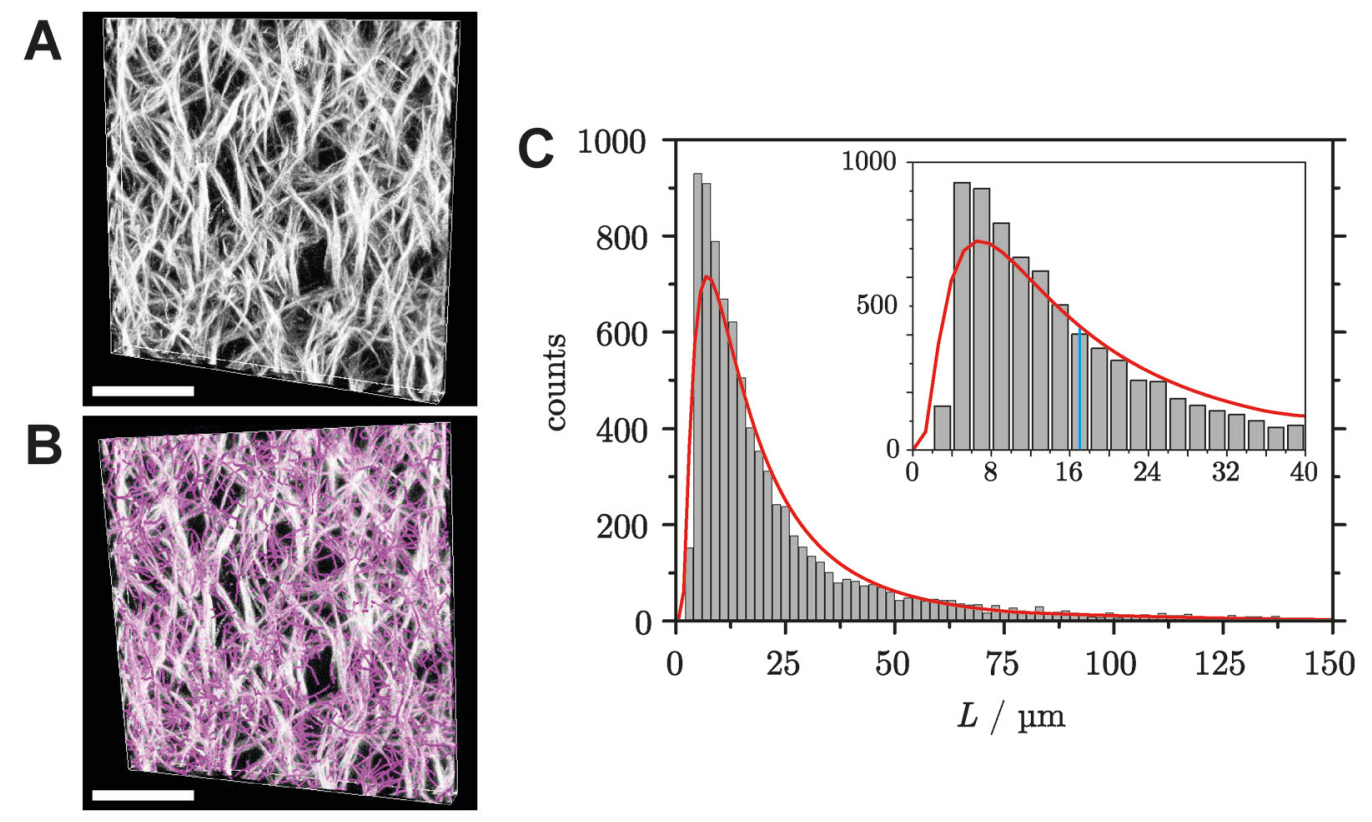

Figure 4.12: Determination of F-actin filament length $(L)$ in $3 D$ actin gels by means of SOAX. A/B: Snapshots during SOAX evaluation showing the $z$-stack rendered to a $3 \mathrm{D}$ image (A) and subsequently with identified chains illustrated in magenta (B). C: The detected filament lengths of three independent experiments were plotted as distribution histogram and a log-normalfit (red) was applied to determine an average filament length of $(17 \pm 3) \mu \mathrm{m}$ (median, inlet: blue line). Scale bars: $30 \mu \mathrm{m}$. 
The obtained filament lengths were plotted as illustrated in Figure 4.12 C. They result in a left-skewed distribution and a log-normal function (equation 4.2) is fit to the histogram data utilizing MATLAB.

$$
f(x)=\frac{1}{x \cdot \sigma \cdot \sqrt{2 \pi}} \cdot \exp \left(-\frac{1}{2 \cdot \sigma^{2}}(\log (x)-\mu)^{2}\right)
$$

Fitting equation 4.2 to the data obtained two parameters: $\mu$, the mean value, and the standard deviation $\sigma$. The mean value $\mu$ of filament lengths was determined to be $2.82 \mu \mathrm{m}$ and the standard deviation $\sigma$ to be $0.943 \mu \mathrm{m}$. When presenting log-normal fitted data, there is an advantage in showing the "back-transformed" data as these values are the corresponding ones to normally distributed data. The "back-transformed" mean value $\mu$ leads to the median $\mu^{*}$ (equation 4.3 ), as well as the standard deviation $\sigma$ leads to the multiplicative standard deviation $\sigma^{*}$ (equation 4.4).[195]

$$
\begin{aligned}
\mu^{*} & =e^{\mu} \\
\sigma^{*} & =e^{\sigma}
\end{aligned}
$$

This is done because for normally distributed data the confidence interval $\mu \pm \sigma$ covers a probability of $68.3 \%$, while $\mu \pm 2 \sigma$ covers $95.5 \%$. In log-normal quantities the corresponding parameters for the confidence intervals are $\left[\mu^{*} / \sigma^{*}, \mu^{*} \cdot \sigma^{*}\right]$ for $68.3 \%$ or $\left[\mu^{*} /\left(\sigma^{*}\right)^{2}, \mu^{*} \cdot\left(\sigma^{*}\right)^{2}\right]$ for $95.5 \%$. Therefore, typically the geometric mean value $\mu^{*}$ and the multiplicative standard deviation $\sigma^{*}$ is given. ${ }^{[195]}$ In this case, this amounts to a filament length of $(17 \pm 3) \mu \mathrm{m}\left(\mu^{*} \pm \sigma^{*}\right)$ with a confidence interval of $[6.5 \mu \mathrm{m}$, $43.1 \mu \mathrm{m}]$ covering a probability of $68.3 \%$. This detected value corresponds well with those presented in literature, where in vitro filament lengths of actin was observed to be in a range of 10 to $20 \mu \mathrm{m} .{ }^{[196,197]}$ Gittes et al. even showed an in vitro F-actin persistence length of about $17.7 \mu \mathrm{m}$ which is nearly the same as the detected value in this work. ${ }^{[196]}$

\subsubsection{Characterization of filament thicknesses by means of AFM}

- Experiments were performed in cooperation with Helen Nöding as part of project A17 of the collaborative research center $937^{[166]}$

F-actin structures in 3D gels already appeared to present in different thicknesses, which was indicated by varying fluorescence intensities between single filaments. In order to distinguish between the presence of single filaments or filament bundles, pre-polymerized networks were analyzed by means of atomic force microscopy (AFM). Specimens were prepared as described in chapter 3.3.5 using pre-polymerized F-actin gels at a concentration of $8 \mu \mathrm{M}$. AFM measurements were conducted in quantitative 
imaging (QI) mode using a JPK Nanowizard 4 (JPK Instruments, Berlin, Germany) as described in chapter 3.5.1.

In this setup, F-actin network gels were adhered to poly-D-lysin surfaces. These cationic surfaces are commonly used in tissue culture, to attach negatively charged cells. ${ }^{[198]}$ Due to the fact that actin filaments can bind to positively charged liposomes, the electrostatic interaction was used to adhere F-actin to poly-D-lysin, in order to avoid displacement caused by the cantilever. ${ }^{[103]}$ Attaching the filaments is therefore advantageous as imaging the height of resulting structures by means of AFM reveal the filament diameter (cf. Figure $4.13 \mathrm{~A}$ ). In a first approach, only $1 \mu \mathrm{L}$ of the pre-polymerized F-actin gel $(c=8 \mu \mathrm{M})$ was added to the surface to obtain single filamentous structures (Figure $4.13 \mathrm{~A}$ ). By imaging single filaments, the suitability of the experimental setup was checked. After single filamentous structures were identified, line scans perpendicular to the found structures (indicated in Figure $4.13 \mathrm{~A}$ ) revealed the detected height, which corresponds to filaments thicknesses (Figure 4.13 B). Single filaments (Figure 4.13 B, yellow box) as well as small bundles (Figure 4.13 B, cyan box) were found. Averaging $N$ line scans, filament heights or diameters of $(7.9 \pm 2.4) \mathrm{nm}(N=18$, yellow $)$ and $(19.1 \pm 2.6) \mathrm{nm}(N=10$, cyan $)$ were determined.
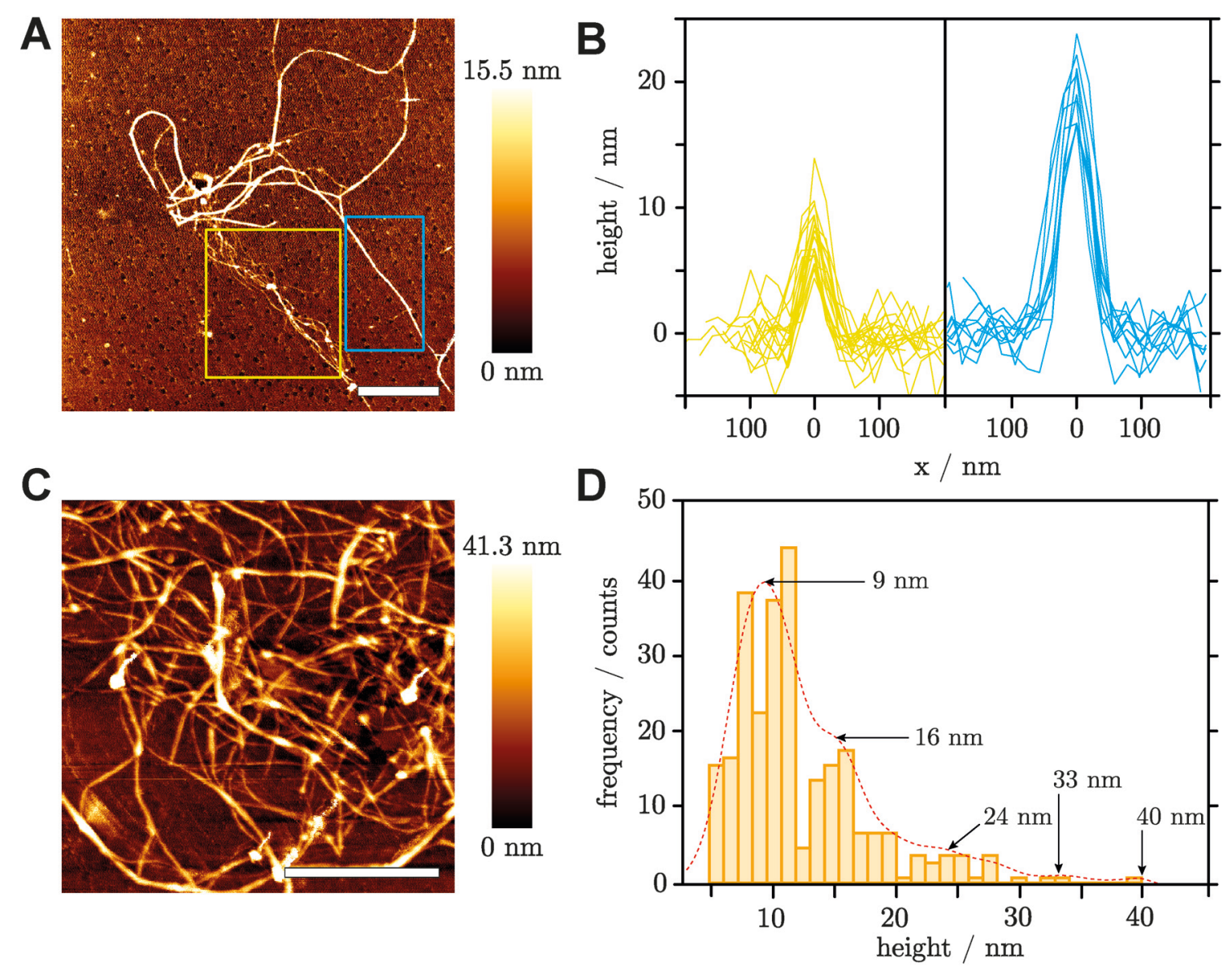

Figure 4.13: Analysis of F-actin filament thicknesses by means of AFM.[160] A: A QI micrograph of an isolated F-actin structure attached to a poly-D-lysine surface $(1 \mu \mathrm{L} \mathrm{F}$-actin gel). Single filamentous structures were chosen (yellow and blue frames) to evaluate 
perpendicular line scans (B). B: Several perpendicular line scans were evaluated to obtain an average diameter of $(7.9 \pm 2.4) \mathrm{nm}(N=18)$ in case of the filamentous structure framed in yellow. Similarly, perpendicular line scans of the filamentous structure framed in cyan revealed a diameter of $(19.1 \pm 2.6) \mathrm{nm}(N=10)$. C: Further QI micrograph of a whole attached network structure $(10 \mu \mathrm{L} \mathrm{F}$-actin gel). D: Histogram of the distribution of the obtained diameters of attached actin filaments $(N=277)$. A probability density fit revealed maxima as shown in the diagram. Primarily, filaments with the diameter of $9 \mathrm{~nm}$ and $16 \mathrm{~nm}$ were found, but also rarely larger diameters of 24, 33 and $40 \mathrm{~nm}$. Modified and reprinted with permission from Nöding, Schön et al. Copyright 2018 American Chemical Society. ${ }^{[160]}$

In subsequent experiments, the poly-D-lysin surface was incubated with $10 \mu \mathrm{L}$ of the pre-polymerized F-actin gel, resulting in whole attached networks (cf. Figure 4.13 C) instead of isolated filamentous structures (cf. Figure 4.13 A). An exemplary QI image of an attached network in shown in Figure $4.13 \mathrm{C}$. For analysis, each image was divided into 49 squares, each filament was recorded once per square and the diameters were presented in one histogram (cf. Figure $4.13 \mathrm{D}$ ). With a probability of $64 \%$ filaments with an average diameter of around $9 \mathrm{~nm}$ were found. Nearly the same thicknesses of 7-9 nm was previously determined in literature for single actin filaments, ${ }^{[5,24,96]}$ which suggests that in the used preparations roughly two third of the structures are present as single filaments. Besides that, an accumulation around $16 \mathrm{~nm}$, which corresponds to two times a single filament, is observable. These bundles consisting of two filaments occur with a probability of around $27 \%$. Only $9 \%$ of the observed bundles were even thicker.

\subsubsection{Attachment of F-actin Networks to Lipid Bilayers}

As a next step, these pre-polymerized F-actin network gels were attached to an ezrin decorated lipid bilayer to form a minimal actin cortex (MAC) as described in detail in chapter 3.3.6. Briefly, a lipid bilayer, containing POPC and PtdIns[4,5] $\mathrm{P}_{2}$, was spread onto a hydrophilic silicon dioxide surface and the active ezrin T567D mutant was bound to the receptor lipid PtdIns[4,5] $\mathrm{P}_{2}$.

In order to exclude an unspecific interaction between the matrix lipid POPC and the F-actin network, a pure POPC bilayer was spread onto a hydrophilic substrate, ezrin T567D and subsequently the pre-formed F-actin network was incubated with the lipid bilayer (cf. Figure 4.14). 

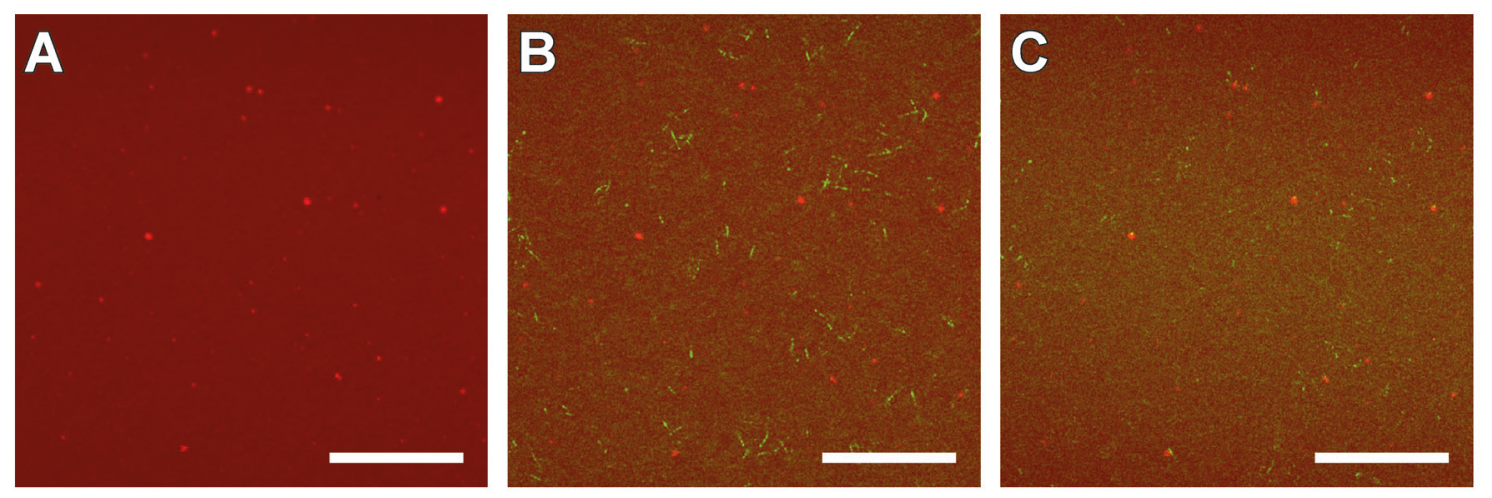

Figure 4.14: Fluorescence micrographs of F-actin attachment to an artificial bilayer without receptor lipid. A: A solid supported lipid bilayer consisting of POPC/TxR-DHPE (99.6:0.4) is showing a red uniform fluorescence signal. Some brighter spots, originating from lipid material, can be observed on top of the bilayer. B: A fluorescence overlay of green Alexa Fluor ${ }^{\circledR} 488$ (F-actin) and red TxR fluorescence intensity (lipid bilayer) is shown. After F-actin incubation, a few filamentous structures can be observed. C: After rinsing the substrate with an Eppendorf pipette almost all filamentous structures are gone. Only a few bright spots are visible in the overlay. Scale bars: $20 \mu \mathrm{m}$.

As shown in chapter 4.1.3 ezrin does not bind to a pure DOPC bilayer, but incubation was nevertheless carried out to maintain the experimental conditions, to enable comparable experiments when using PtdIns[4,5] $\mathrm{P}_{2}$. Figure 4.14 shows that a successfully spread lipid bilayer could be observed (cf. Figure 4.14 A), and that after F-actin incubation small filaments become visible (Figure $4.14 \mathrm{~B}$ ). Afterwards, the specimen was rinsed, and the same spot was recorded again to observe possible changes. The fluorescence micrograph shown in Figure 4.14 C demonstrates the situation after rinsing. The membrane appears similar to Figure $4.14 \mathrm{~A} / \mathrm{B}$ because the brighter spots, originating from lipid material, remain at their position, as observed in the red TxR-DHPE fluorescence of the micrographs. In green fluorescence less filament fragments, in contrast to Figure $4.14 \mathrm{~B}$, were observed. Therefore, the filament fragments in Figure 4.14 B are flushed away due to rinsing. No attachment of an F-actin network to a lipid bilayer in absence of $\operatorname{PtdIns}[4,5] \mathrm{P}_{2}$ could be detected in three independent experiments.

In contrast to the experiments presented in Figure 4.14, a clearly attached F-actin network morphology could be realized when PtdIns [4,5] $\mathrm{P}_{2}$ was present in the lipid bilayer. The PtdIns[4,5] $\mathrm{P}_{2}$ ratio inside the lipid bilayer was altered from 1-8 mol \%, like it was already done for characterization of ezrin T567D binding (cf. chapter 4.1.3). Exemplary fluorescence micrographs of an F-actin network attached to a lipid bilayer with $3 \mathrm{~mol} \% \operatorname{PtdIns}[4,5] \mathrm{P}_{2}$, recorded after rinsing the specimen, are shown in Figure 4.15. 


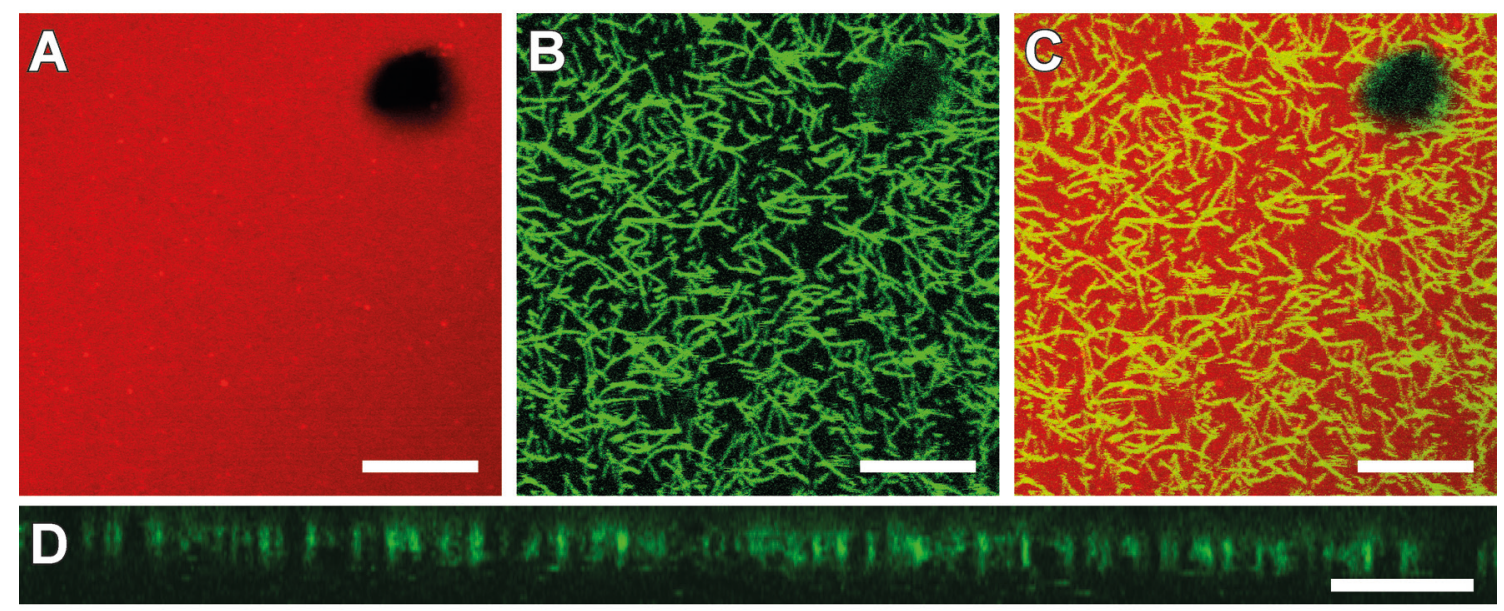

Figure 4.15: Fluorescence micrographs showing an F-actin network attached to an ezrin decorated lipid bilayer.[160] A: A lipid bilayer composed of DOPC/PtdIns[4,5] $\mathrm{P}_{2} /$ TxR-DHPE (96.6:3:0.4) was spread on a hydrophilic silicon surface. Besides the homogeneous red membrane fluorescence, a black spot in the upper right corner is detected showing a membrane defect. B: The attached filamentous actin network, labeled with Alexa Fluor ${ }^{\circledR}$ 488-phalloidin, is visible as green structures forming a network morphology. C: An overlay of $(\mathbf{A})$ and $(\mathbf{B})$ reveals that no F-actin structures are found in the membrane-less area of the membrane defect. D: A cross section of a $z$-stack shows the height of the MAC. Total z-stack image height: $4 \mu \mathrm{m}$. Scale bars: A-C: $5 \mu \mathrm{m}$, D: $2 \mu \mathrm{m}$. Modified and reprinted with permission from Nöding, Schön et al. Copyright 2018 American Chemical Society.[160]

In Figure 4.15 A, the TxR-DHPE fluorescence signal of the lipid bilayer is shown, while $\mathrm{B}$ displays the fluorescence signal of the F-actin network. In the upper right corner of the membrane fluorescence, a black spot is observed showing a membrane-less area or a membrane defect (cf. Figure 4.15 A). This defect is also observed in the fluorescence micrograph of the F-actin network (cf. Figure $4.15 \mathrm{~B}$ ). Clearly to see in the overlay of both fluorescence signals (cf. Figure $4.15 \mathrm{C}$ ), the F-actin network also leaves out the membrane defect neither spanning over this area nor binding to the hydrophilic surface. Therefore, a specific binding to ezrin, which is bound to the lipid bilayer, is suggested because the F-actin network neither binds to the hydrophilic surface of the silicon substrate (cf. Figure 4.15 A-C) nor to the matrix lipid DOPC without any receptor lipid (Figure 4.14). A successful formation of a minimal actin cortex (MAC) is achieved. Interestingly, the length of the MAC's filaments appears to be shorter than the observed length of 3D F-actin networks gels (cf. Figure 4.10).

Furthermore, a cross section of recorded z-stacked images reveals a flat layer of the network (cf. Figure 4.15 D). In the cross section, a clear plane of wide-spread points is observed, which were suggested to originate from crosswise cut filamentous structures (green, bright spots) and pockets (dark areas) of the network. The green fluorescence signal of the plane extents around $1 \mu \mathrm{m}$ in height. 
Figure 4.15 is only one example of an MAC, formed with a lipid bilayer containing $3 \mathrm{~mol} \%$ PtdIns $[4,5] \mathrm{P}_{2}$. As already mentioned, PtdIns $[4,5] \mathrm{P}_{2}$ ratio was varied from $1-8 \mathrm{~mol} \%$ and in chapter 4.1 .3 , it was presented that the ezrin surface coverage increases with increasing $\mathrm{PtdIns}[4,5] \mathrm{P}_{2}$ ratio, the defined pinning point density. Therefore, MAC attachment was investigated in dependence of the ezrin surface coverage which was adjusted by the pinning point density. Fluorescence micrographs were recorded to study the changing network morphology of the MACs. Some exemplary MACs with different pinning point densities are shown in Figure 4.16. In total, florescence micrographs of 1,3,4,5,6,7 and $8 \mathrm{~mol} \%$ were taken, but not shown for clarity. Worthy of mention, confocal fluorescence micrographs differ a lot in appearance. Not only the signal-to-noise ratio can be different (compare Figure 4.16 A and B), also the resolution was improved by changing from FV 1200 (Olympus Europe $S E \&$ Co.KG, Hamburg, Germany) to LSM 880 with Airyscan technology (Carl ZeisS Microscopy GmbH, Oberkochen, Germany). One exemplary Airyscan image is shown in Figure 4.16 D.

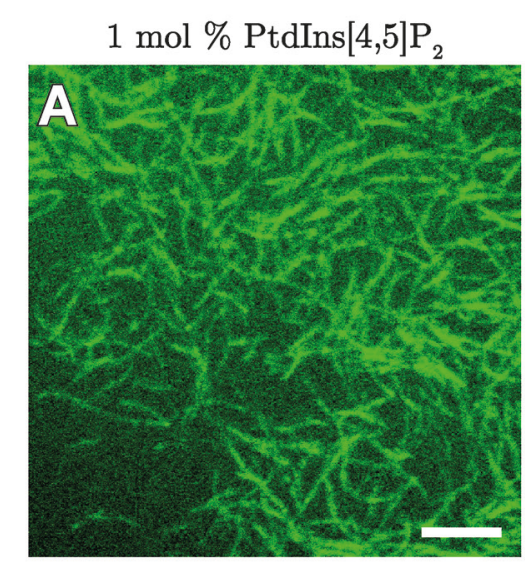

5 mol \% PtdIns $[4,5] \mathrm{P}_{2}$

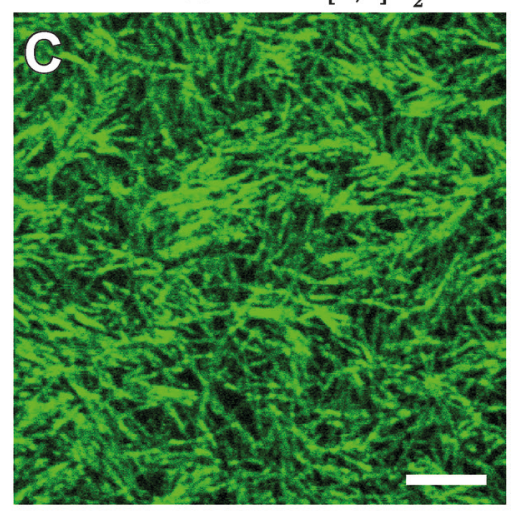

$3 \mathrm{~mol} \% \mathrm{PtdIns}[4,5] \mathrm{P}_{2}$

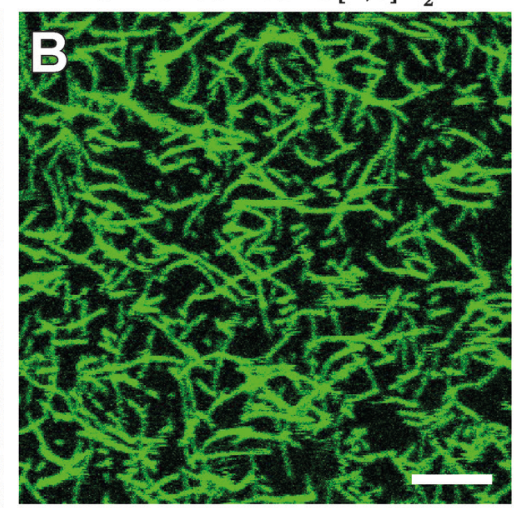

$8 \mathrm{~mol} \% \operatorname{PtdIns}[4,5] \mathrm{P}_{2}$

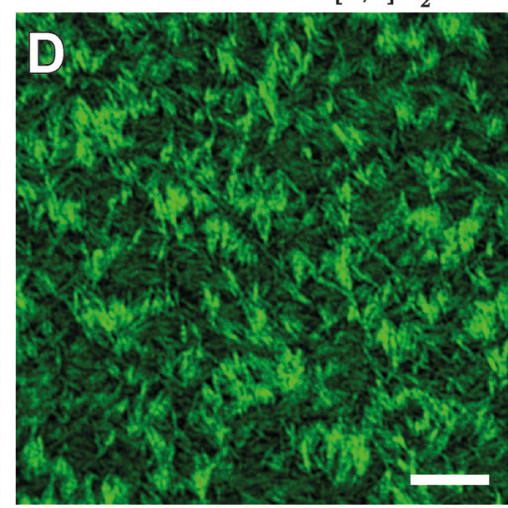

Figure 4.16: Exemplary network morphologies of attached MACs with different pinning point densities. A: An MAC labeled with Alexa Fluor ${ }^{\circledR}$ 488-phalloidin on an ezrin-decorated lipid bilayer with only $1 \mathrm{~mol} \% \operatorname{PtdIns}[4,5] \mathrm{P}_{2}$ shows a layer of bound F-actin structures. Some parts of the substrate are free of F-actin as shown in the lower left corner. B: With increased pinning point density of $3 \mathrm{~mol} \% \operatorname{PtdIns}[4,5] \mathrm{P}_{2}$ in the lipid bilayer it can be observed that areas free of $\mathrm{F}$-actin disappear and the whole substrate is covered with F-actin. C: A further increase in pinning point density leads to a denser network, as it is suggested by 
the naked eye. D: At $8 \mathrm{~mol} \% \operatorname{PtdIns}[4,5] \mathrm{P}_{2}$, the network gets so dense that the network morphology appears to be fan-shaped in some cases. The resulting filaments appear to be shorter, and the MAC seems to have two levels in intensity suggesting more than one F-actin layer. However, a higher cortex thickness could not be verified by z-stacked images. Notably, only (D) is an Airyscan image. Scale bars: $5 \mu \mathrm{m}$.

The detected morphology of the MACs differs a lot dependent on the change in pinning point density by stepwise increase of the PtdIns $[4,5] \mathrm{P}_{2}$ content in the lipid bilayer. At $1 \mathrm{~mol} \% \operatorname{PtdIns}[4,5] \mathrm{P}_{2}$, it is obvious that the ezrin surface coverage is not enough as the F-actin network is not able to cover the substrate with a complete layer (Figure $4.16 \mathrm{~A})$. Thus, the offered links by ezrin are too less to attach the network to the lipid bilayer. Increasing the pinning point density led to an MAC that covers the whole substrate as shown in Figure 4.16 B. A further increase of pinning point density via $5 \mathrm{~mol} \%$ (Figure $4.16 \mathrm{C}$ ) up to $8 \mathrm{~mol} \% \operatorname{PtdIns}[4,5] \mathrm{P}_{2}$ changed the morphology to denser networks. The micrograph shown in Figure $4.16 \mathrm{D}$ appears to be very special. At molar ratios of $8 \mathrm{~mol} \% \operatorname{PtdIns}[4,5] \mathrm{P}_{2}$ a fan-shaped morphology, like the one shown here, was sometimes observed. These structures were only observable in a few experiments but appeared only in experiments with $8 \mathrm{~mol} \% \operatorname{PtdIns}[4,5] \mathrm{P}_{2}$, thus with a large pinning point density. The fluorescence signal seems to be in two levels, one darker level and one with fan-shaped highlighted structures on top. However, a higher cortex thickness could not be verified by z-stacked images. Considering all recorded z-stacked images in general, there was no dependence of pinning point density and cortex height found. To evaluate the cortex height, the extension of the fluorescence signal in $z$-direction was plotted which results in a normal distribution. The full width at half maximum (FWHM) of this distribution is determined, resulting in a mean value of $(1.5 \pm 0.3) \mu \mathrm{m}$. This mean value was determined using all $z$-stacked images of fluorescence micrographs of F-actin networks, no matter which $\operatorname{PtdIns}[4,5] \mathrm{P}_{2}$ ratio, because no dependence between the different ratios could be observed. It must be considered that the point-spread-function in $z$-direction is quite large which reduces the axial resolution under $1 \mu \mathrm{m}$. The Rayleigh limit shows that two points closer than $700 \mathrm{~nm}$ will appear as one, at axial scales using the wavelength and the microscopic setup of this work (cf. chapter 3.5.2).[147] This suggests that the MAC is thinner than $1 \mu \mathrm{m}$. The detected thickness is on the same magnitude as the average cell cortex height of living cells which is about $200 \mathrm{~nm}$. Furthermore, it was shown that the cortex height is independent of membrane-cortex attachment by depletion experiments of ERM proteins. ${ }^{[4]}$ 


\subsubsection{Analysis of Self-Organization of attached MACs}

Fluorescence micrographs shown in Figure 4.16 indicate that the obtained MACs get denser with increasing pinning point density. To verify this hypothesis, it was necessary to get access to quantitative parameters of the network morphology. Some typical parameters of biopolymer networks used in literature are displayed in Figure 4.17.

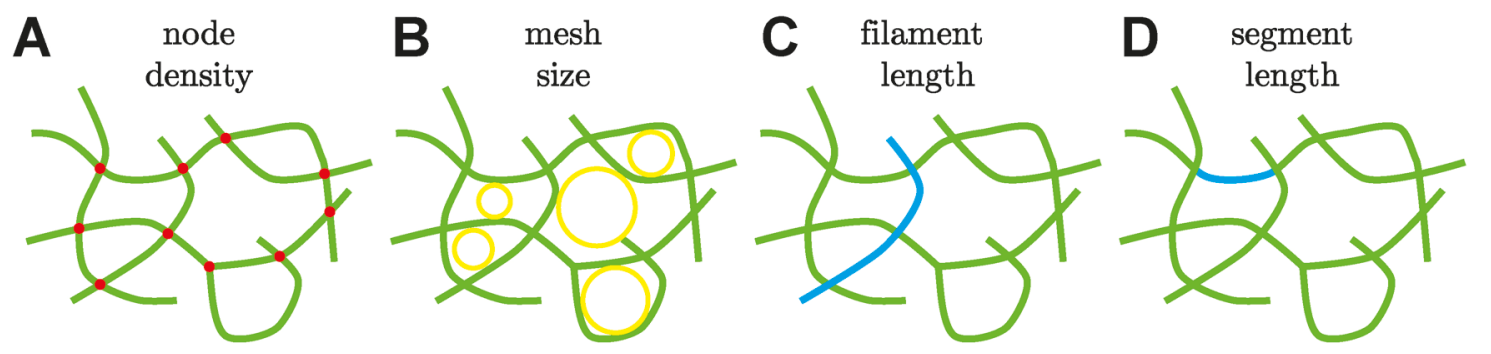

Figure 4.17: Schematic illustration of quantitative network parameters. A: Filaments crossing each other or bundles partitioning into two filaments have entanglement points or network nodes. Calculating the sum of nodes per square micron indicates a node density related to each network. B: The resulting network displays pockets between filaments which relate to the so-called mesh size. C-D: The contour length of the whole filament or bundle as well as the length of a segment between two nodes (highlighted in cyan) are also quantitative parameters of each network.

This work focuses on the node density and mesh size, two parameters which should give a clear hint about the density of the imaged actin cortex. In this case, nodes were defined as all kind of entanglement points: junction points as well as intersections of two actin filaments. A distinct number of nodes per specific area e.g. nodes per $\mu^{2}$ delivers a comparable network parameter. Additionally, the mesh size, respectively the arising pockets between the actin filaments are also specific for the resulting network morphology and show also the density of a network. Since these network pockets arise in different geometries, for example a wide rectangle in contrast to a square pocket, these pockets are hard to evaluate. In literature, biopolymer network pockets were evaluated by finding the one special circle (in literature so-called "bubble") which has the largest diameter and is still fitting into the pocket.[164] This method will also be utilized in this work. Furthermore, the whole filament contour lengths (Figure 4.17 C) and segment lengths (Figure $4.17 \mathrm{D}$ ) which is the length between two nodes or ends, will be evaluated in the subsequent parts.

Therefore, the most important step to analyze these quantitative parameters is to convert the confocal fluorescence micrograph into a skeleton which describes the network morphology. Different signal-to-noise ratios and image resolutions ranging from $25 \mathrm{~nm} \cdot \mathrm{px}^{-1}$ up to $110 \mathrm{~nm} \cdot \mathrm{px}^{-1}$ challenge every routine of skeletonization. In this work, two pathways of skeletonization were utilized: On the one hand, networks were skeletonized with the software SOAX, on the other hand skeletonization was achieved 
via processing with the artificial retina. Both methods are described in detail in chapter 3.6.1. In order to decide which method works best with the shown fluorescence micrographs, both methods, using a small data set of three different PtdIns $[4,5] \mathrm{P}_{2}$ ratios, were compared.

The small data set consists of: $\quad N\left(\operatorname{PtdIns}[4,5] \mathrm{P}_{2}=1 \mathrm{~mol} \%\right)=5$, $N\left(\mathrm{PtdIns}[4,5] \mathrm{P}_{2}=3 \mathrm{~mol} \%\right)=12, N\left(\mathrm{PtdIns}[4,5] \mathrm{P}_{2}=1 \mathrm{~mol} \%\right)=12$ with $N$ as the number of fluorescence micrographs.

\section{Network Skeletonization using the software SOAX or the Artificial Retina}

The open source software SOAX is based on the principle of evolving initialized "stretching open active contours" (SOACs) on an FFT (fast Fourier transform) filter processed fluorescence micrograph as described in detail in chapter 3.6.1.2. This software provides a computed skeleton as well as the detected filament contour length (cf. Figure 4.18 B1). The optimized parameter set which was used for an analysis routine can be found in the appendix (chapter 7.2). The mesh size of the network was analyzed using the "bubble analysis" applied to the resulting skeleton as presented in literature. This analysis utilizes an Euclidian distance map (EDM) and was employed using the software MATLAB. ${ }^{[164,165]}$ It is further described in chapter 3.6.3. An exemplary evaluation is illustrated in Figure 4.18 on the left side (1) showing the main steps of evaluation.

A second approach of evaluating the morphology of MACs was the use of the artificial retina analysis. Briefly, obtained fluorescence micrographs were processed to a retina image (cf. Figure 4.18 B2, detailed information in chapter 3.6.1.1) which was thresholded and therefore led to a network skeleton which was further evaluated. Filament lengths and number of nodes were directly extracted by the PYTHON script of artificial retina developed by Nils Dörrer (Georg-August-Universität Göttingen). The analysis of mesh sizes was again performed using the "bubble analysis". The described pathway of analyzing a fluorescence micrograph via this method is illustrated in Figure 4.18 on the right side (2). In total, Figure 4.18 displays a comparison of both analysis methods. 

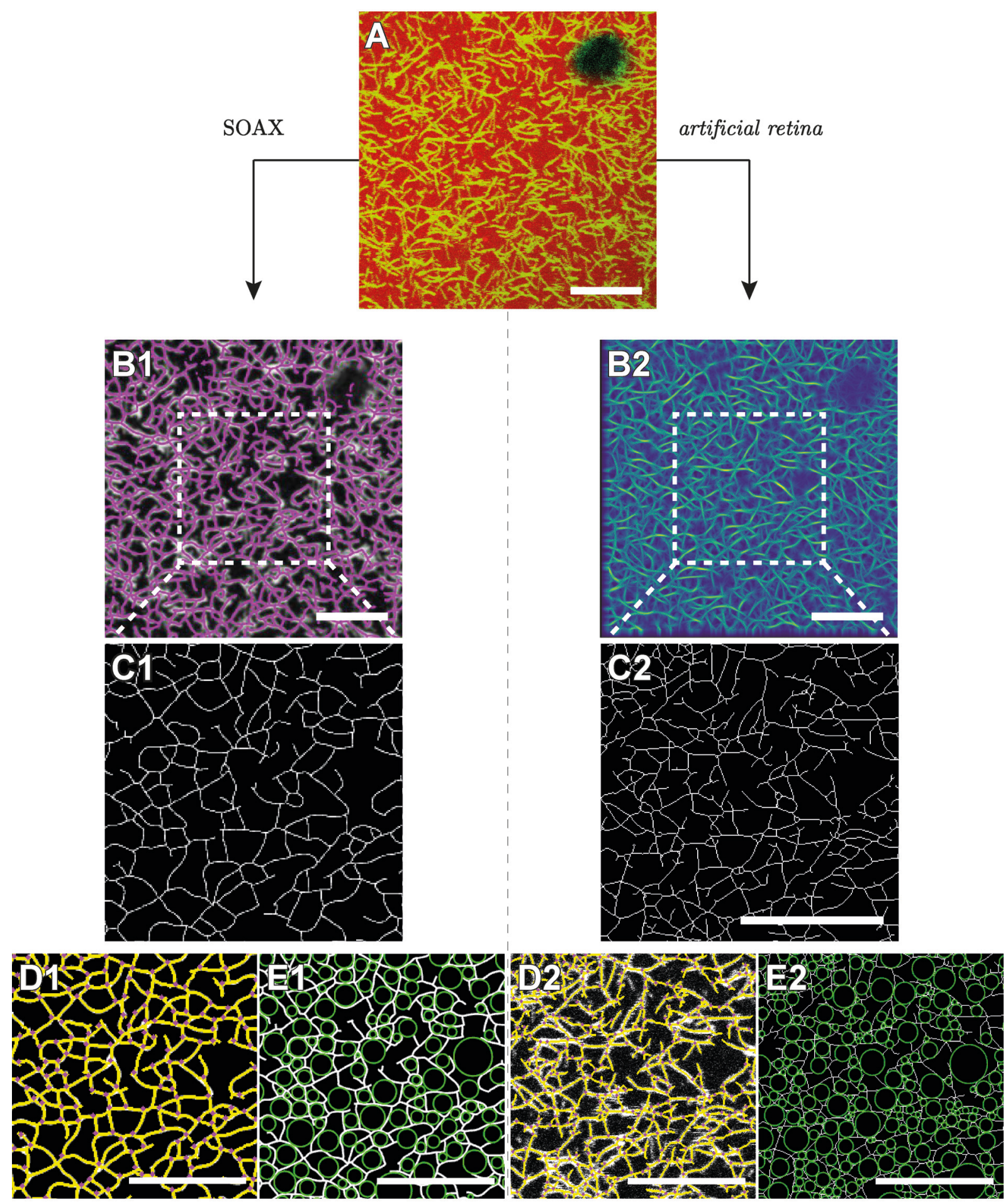

Figure 4.18: Typical pathway of analyzing a fluorescence micrograph using SOAX (1) or the artificial retina (2). A: An exemplary fluorescence micrograph showing an overlay of fluorescence signal of the lipid bilayer (TxR-DHPE, red) and the F-actin network (Alexa Fluor ${ }^{\circledR}$ 488-phalloidin, green). B: The fluorescence micrograph of the actin network only (A, green) was subjected to either a network of computed SOACs (B1, magenta) or the artificial retina revealing a skeletonized or a retina image (B2), respectively. C: While the computed SOACs directly display the network skeleton $(\mathbf{C 1})$ the obtained retina image (B2) was subsequently thresholded to obtain the network skeleton (C2). D-E: Both skeletons were analyzed aiming at the detection of the node density $\left(\mathbf{D}\right.$, nodes per $\left.\mu \mathrm{m}^{2}\right)$ and mesh size $(\mathbf{E}$, circle diameter). Scale bars: $5 \mu \mathrm{m}$. 
Exemplary skeletons (Figure $4.18 \mathrm{C}$ ) resulting from thresholded retina images or SOAXs supply a clear morphologic network structure where the node density (Figure $4.18 \mathrm{D}$, purple nodes on a yellow skeleton) and the mesh size (Figure $4.18 \mathrm{E}$, green circles within the skeleton) can be extracted. Comparing both skeletons among themselves, the skeleton computed with SOAX appears way smoother than the skeleton of the artificial retina. The skeleton of the artificial retina provides many small filaments intersecting the longer filaments and more detailed structures in general. It seems reasonable that the detected values for the quantitative network parameters may differ between both analysis methods. Therefore, both methods and the obtained data must be compared to evaluate which analyzing method suits best for the obtained fluorescence micrographs.

\section{Comparison of SOAX' and Artificial Retina's Data}

First, the length parameters of both methods are compared. The software SOAX only provides the lengths of the detected SOACs but does not supply the direct values for network nodes and the segment lengths. Therefore, the computed filaments were redrawn with the obtained data and the resulting network (denoted as SOAX skeleton) was analyzed. Utilizing PYTHON, the skeletons were thresholded, nodes were determined (cf. chapter 3.6.2), and the resulting fragments in between, the so-called segment lengths, were collected as pixels and recalculated to lengths scales using the image resolution. The filament length was obtained by adding distinct segment lengths: each node has 3 or 4 adjoining fragments. In case of 4 adjoining fragments, the opposite fragments were summed up to get the filament length. In case of 3 adjoining fragments, the two fragments with the greatest angle in between were recognized as belonging together. This construct gets important when a bundled structure branches into two filaments. Detected filament lengths were plotted as a histogram and fitted with a log-normal function (cf. equation 4.2) revealing the median and multiplicative standard deviation. The detected segment lengths for the minimal data set are displayed in Table 4.2, the filament contour lengths in Table 4.3.

Table 4.2: Determined segment lengths of the minimal data set via SOAX's and artificial retina's skeleton.

\begin{tabular}{c|c|c}
$\begin{array}{c}\text { PtdIns[4,5]P } \\
\text { 2 }\end{array}$ & $\begin{array}{c}\text { segment length } / \mathbf{\mu m} \\
\text { [SOAX skeleton] }\end{array}$ & $\begin{array}{c}\text { segment length } / \mathbf{u m} \\
\text { [artificial retina] }\end{array}$ \\
\hline 1 & $1.7 \pm 2.5$ & $1.4 \pm 2.5$ \\
3 & $1.6 \pm 2.9$ & $1.6 \pm 3.0$ \\
5 & $1.5 \pm 2.7$ & $1.2 \pm 2.9$
\end{tabular}


Table 4.3: Determined filament lengths of the minimal data set of SOAX [SOAX], the artificial retina [artificial retina] and the redrawn, thresholded SOAX-skeleton [SOAX skeleton]. $N$ displays the number of found filaments.

\begin{tabular}{|c|c|c|c|}
\hline $\begin{array}{c}\operatorname{PtdIns}[4,5] \mathrm{P}_{2} \\
/ \mathrm{mol} \%\end{array}$ & $\begin{array}{c}\text { filament length } \\
\qquad / \mu \mathrm{m} \\
{[\mathrm{SOAX}]}\end{array}$ & $\begin{array}{l}\text { filament length } \\
\qquad / \mu \mathrm{m} \\
\text { [artificial retina] }\end{array}$ & $\begin{array}{c}\text { filament length } \\
\text { / / } \\
\text { [SOAX skeleton] }\end{array}$ \\
\hline 1 & $\begin{array}{c}21 \pm 2 \\
(N=3191)\end{array}$ & $\begin{array}{l}3.8 \pm 2.5 \\
(N=397)\end{array}$ & $\begin{array}{l}4.3 \pm 2.6 \\
(N=406)\end{array}$ \\
\hline 3 & $\begin{array}{c}26 \pm 2 \\
(N=17640)\end{array}$ & $\begin{array}{l}3.8 \pm 3.5 \\
(N=3157)\end{array}$ & $\begin{array}{l}4.2 \pm 3.4 \\
(N=1600)\end{array}$ \\
\hline 5 & $\begin{array}{c}24 \pm 2 \\
(N=10616)\end{array}$ & $\begin{array}{c}3.4 \pm 3.5 \\
(N=1551)\end{array}$ & $\begin{array}{l}4.1 \pm 2.8 \\
(N=898)\end{array}$ \\
\hline
\end{tabular}

The detected segment lengths are in the same range but have a large (multiplicative) standard deviation $\sigma$. The reason for that can be the heterogeneity of the network morphology. Different appearing meshes are surrounded by filament segments with different lengths. A variety of pocket geometries would therefore explain the large deviation. Furthermore, the detected change between the molar ratios appears to be very small, especially when the error margin is considered. Comparing both methods of skeletonization, the values are in a close range to each other. The detected segment length determined with the method of artificial retina displays smaller lengths. Regarding the skeletons shown in Figure $4.18 \mathrm{D}$, this is reasonable as the retina's skeleton shows more small filamentous fragments due to the thresholding procedure which moves the distribution of segment lengths slightly to smaller length scales. Interestingly, the detected filament contour length shows great differences depending on the evaluation method. While the artificial retina detects filament lengths between 3 and $4 \mu \mathrm{m}$ the software SOAX output filament lengths between 21 and $26 \mu \mathrm{m}$. Due to the fact that the SOAX-skeletons were re-evaluated with PYTHON for segment length analysis anyway, the filament contour length read out with this method was also shown for comparison. In this case, filament lengths of about $4 \mu \mathrm{m}$ were found. That underlines that the detection of filament lengths outputted by SOAX must be a completely different approach. This is supported by the fact that SOAX determines around the 10-fold more filaments overall. Small differences in obtained filaments are reasonable due to different approaches of skeletonization but a 10-fold difference shows significant differences in methodology. The number of found filaments is equal with the number of evolved SOACs. In literature, three reasons can be found why the network reconfiguration of SOAX using the SOACs might not be correct: ${ }^{[163]}$ first, the sequential evolution is only designed for $\mathrm{T}$ - or corner junctions without respecting higher-degree junctions. Second, SOACs evolve one after the other which leads to the fact that earlier evolved SOACs have a better chance to extend along multiple branches or junctions. 
Last, sharp turns may also be interpreted as a SOAC end as they can be influenced by "strong external force fields", such as a very bright branch nearby. ${ }^{[163]}$ In order to illustrate these challenges, an exemplary SOAC evolution on a small image was performed (cf. Figure 4.19). Here, every detected SOAC is drawn in a different color. The problem of SOAC initialization order (reason 2) can be observed in the yellow box of Figure 4.19 B. The red illustrated filament is clearly divided by the blue and the cyan filament. By human eye one would assume that the red filament should be counted as one as well as the blue and cyan filament belongs together. Furthermore, also a sharp turn close to a bright branch is observed (cf. Figure 4.19

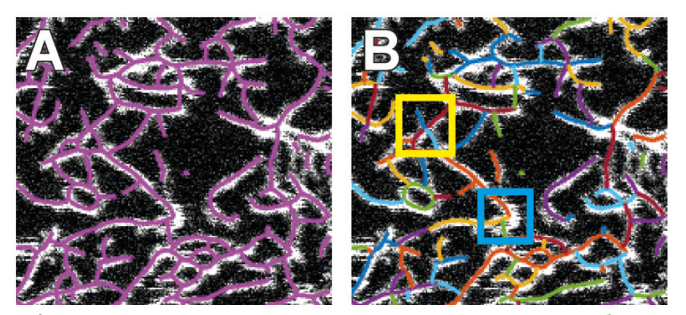

Figure 4.19: Exemplary comparison of the detected SOAX skeleton (A) and the skeleton filaments assorted to colors (B).

B cyan box). As postulated in literature, SOAX divides the filament into the orange and yellow one although one would assume that it could be one filament. These reasons are the main origin why SOAX is detecting more filaments as the artificial retina analysis. The second conspicuity is that the higher mean value of the filament length can originate from two reasons: First, the detected SAOCs must be longer than the detected filaments of the artificial retina across the board. This would cause a shift of the whole distribution to longer filament lengths. The second possibility is that all the short fragments, which are detected using the artificial retina, are missing in the skeleton of SOAX. This possibility seems to be very likely, since one starting parameter of the SOAX evaluation is setting up a minimal snake length. This parameter was set to five pixels because in case of three pixels too many terminations of the SOAC evolution were observed. Sensitive to the image resolution, this already would exclude many small fragments which are recorded by the method of artificial retina. Last, the first mentioned challenge of the disrespecting of higher-degree junctions or branches is a complication which both methodologies of skeletonization must deal with as also the analysis via artificial retina gets inaccurate in case of 5 or more adjoining fragments.

Because the length parameters of the skeletons mostly show differences between both methods instead of differences between the different $\mathrm{PtdIns}[4,5] \mathrm{P}_{2}$ ratios, the network morphology was further evaluated in order to obtain the mesh size and node density. The mesh size of both methods' skeletons was evaluated using the "bubble analysis" and the resulting mesh size diameters are shown in Figure 4.20. 

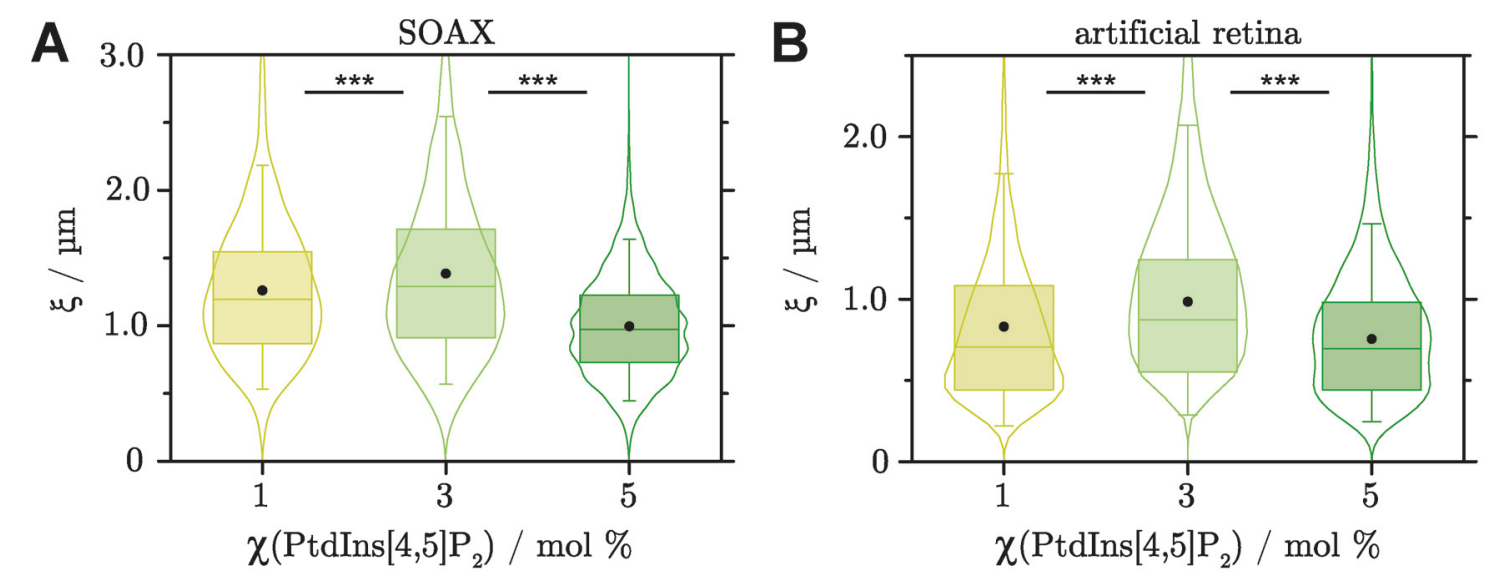

Figure 4.20: Violin plot of the resulting mesh sizes $\xi$ (circle diameter) of network pockets dependent on skeletonization method. A: The mean values of detected mesh sizes utilizing the SOAX skeleton were all above $1 \mu \mathrm{m}$, showing a clear tendency to smaller meshes from $3 \mathrm{~mol} \%$ to $5 \mathrm{~mol} \% \operatorname{PtdIns}[4,5] \mathrm{P}_{2}$. B: The meshes of the artificial retina's skeleton show the same tendency. In both cases the mesh size of networks attached to 1 mol \% PtdIns $[4,5] \mathrm{P}_{2}$ show a smaller mesh size than 3 mol \% PtdIns $[4,5] \mathrm{P}_{2}$. Mann-Whitney $U$ tests were performed to show that two populations are not populations with equal means. Significance levels are: $5 \%\left(^{*}\right), 1 \%(* *)$ and $0.1 \%\left(^{* *}\right)$. Box plots extent from $25^{\text {th }}$ to $75^{\text {th }}$ percentile and whiskers from $5^{\text {th }}$ to $95^{\text {th }}$. The violin body represents the kernel density function of the mesh size distribution.

The determination of the mesh size $\xi$ reveals similar tendencies for both methods of network skeletonization. In general, the mesh size obtained by SOAX skeleton is larger than the one obtained by the artificial retina's skeleton. While SOAX displays mean values of mesh sizes from $(1.3 \pm 0.5) \mu \mathrm{m}\left(\chi_{\mathrm{PtdIns}[4,5] \mathrm{P}_{2}}=1 \mathrm{~mol} \%\right)$ via $(1.4 \pm 0.6) \mu \mathrm{m}$ $\left(\chi_{\mathrm{PtdIns}[4,5] \mathrm{P}_{2}}=3 \mathrm{~mol} \%\right)$ to $(1.0 \pm 0.4) \mu \mathrm{m}\left(\chi_{\mathrm{PtdIns}[4,5] \mathrm{P}_{2}}=5 \mathrm{~mol} \%\right)$, the artificial retina shows mean values of $(0.8 \pm 0.5) \mu \mathrm{m},(1.0 \pm 0.6 \mu \mathrm{m}$ and $(0.8 \pm 0.4) \mu \mathrm{m}$ (cf. Figure 4.20). Thus, SOAXs show mesh size above $1 \mu \mathrm{m}$ while all mesh sizes of the artificial retina are beyond $1 \mathrm{\mu m}$, but both methods show the same tendency between the molar ratios of $\operatorname{PtdIns}[4,5] \mathrm{P}_{2}$. Concerning the comparison of the skeletons shown in Figure $4.18 \mathrm{C}$, this tendency is not surprising as the artificial retina's skeleton provides the already mentioned little fragments intruding the network pockets. This intruding filament segments reduces the diameter of the resulting best-fitting circle inside the pocket which therefore decreases the determined mesh size. Additionally, the presented violin bodies deriving from the kernel density function of all detected mesh sizes show a very broad distribution of mesh sizes (cf. Figure 4.20). This underlines the heterogeneity of the F-actin network morphology. In case of the artificial retina's skeleton this distribution appears to be slightly broader than the mesh size of SOAX's skeleton. Both data sets indicate that a larger data set for final network evaluation is needed.

The last and most promising network parameter is the node density. While the analysis of mesh size is a good indicator for the density of networks, it faces the already $-98-$ 
mentioned problem of different appearances of network pocket which are finally reduced to one single circle with the largest diameter. The network junction points, no matter if entanglement, cross-over or branching point, are a clear parameter for the network's density. In contrast to the mesh size, the nodes are comparatively easy to evaluate (cf. chapter 3.6.2). A comparison of node densities obtained from SOAX and artificial retina is displayed in Figure 3.21.
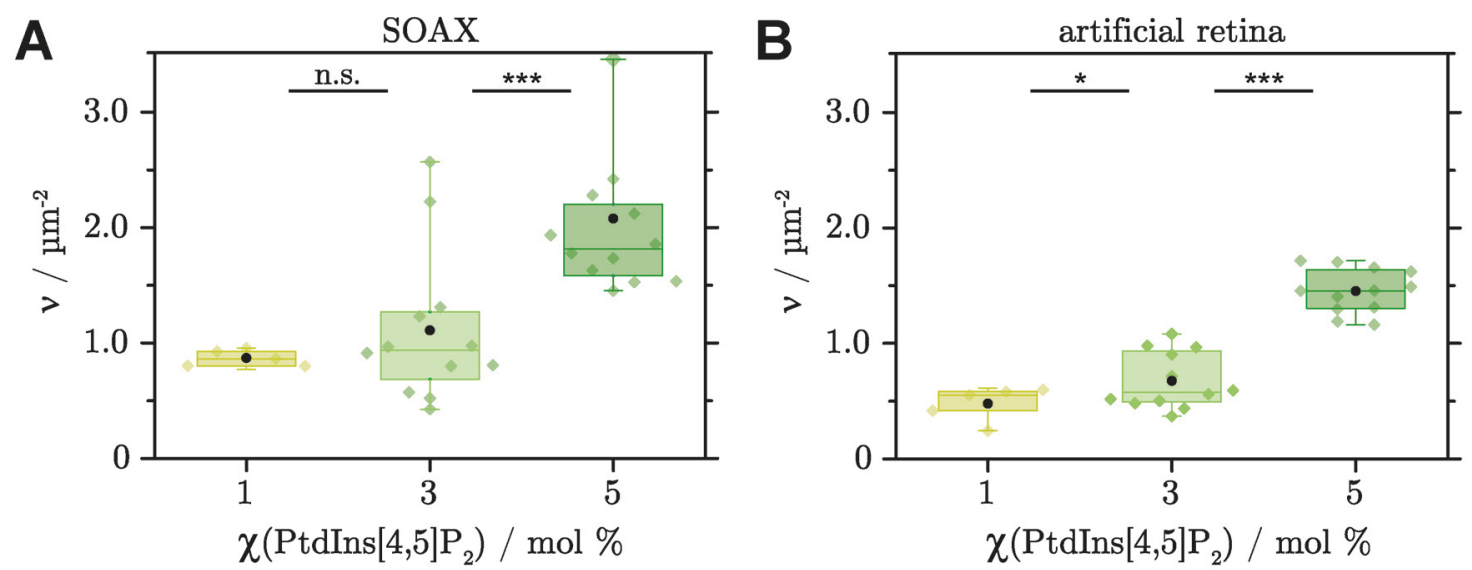

Figure 4.21: Box plots representing the node density $v$ (nodes per $\mu^{2}{ }^{2}$ ) of network skeletons dependent on the method of skeletonization. A: Node density of SOAX's network skeletons shows a continuous increase in node density with increasing pinning point density. B: The same dependence can be observed in case of artificial retina's skeletons. The determined node density is generally smaller than the presented node density in $(\mathbf{A})$. Furthermore, the distribution is narrower. Mann-Whitney $U$ tests were performed to show that two populations are not populations with equal means. Significance levels are: $5 \%(*), 1 \%$ $(* *)$ and $0.1 \%(* * *)$ while n.s. displays two non-significant data sets. Box plots extent from $25^{\text {th }}$ to $75^{\text {th }}$ percentile and whiskers from $5^{\text {th }}$ to $95^{\text {th }}$. Each node density of a single image is shown in the background of the box plot.

As before, the tendency within the $\mathrm{PtdIns}[4,5] \mathrm{P}_{2}$ ratio of both skeletonization methods is nearly the same. A slight increase from $1 \mathrm{~mol} \%$ to $3 \mathrm{~mol} \%$ is followed by a more apparent increase at $5 \mathrm{~mol} \%$. While the distribution of detected node densities is quite narrow in case of the artificial retina's skeletons, it is surprisingly wide spread at SOAX's skeletons. Considering the skeleton of the artificial retina showing many of the mentioned little filament fragments (Figure $4.22 \mathrm{C}$, yellow box), it was assumed that these skeletons show a higher node density than SOAX's skeletons as every fragment comes along with an additional node. One possible explanation could be that the computation of the SOAX "snakes" often bridges between two filaments establishing a connection where the fluorescence micrograph does not obviously show a connection. This connection is therefore classified differently by naked eye or the artificial retina which can be observed in the enlarged skeleton shown in Figure $4.18 \mathrm{D}$ and is compared to the original fluorescence micrograph in Figure 4.22 A-C. 

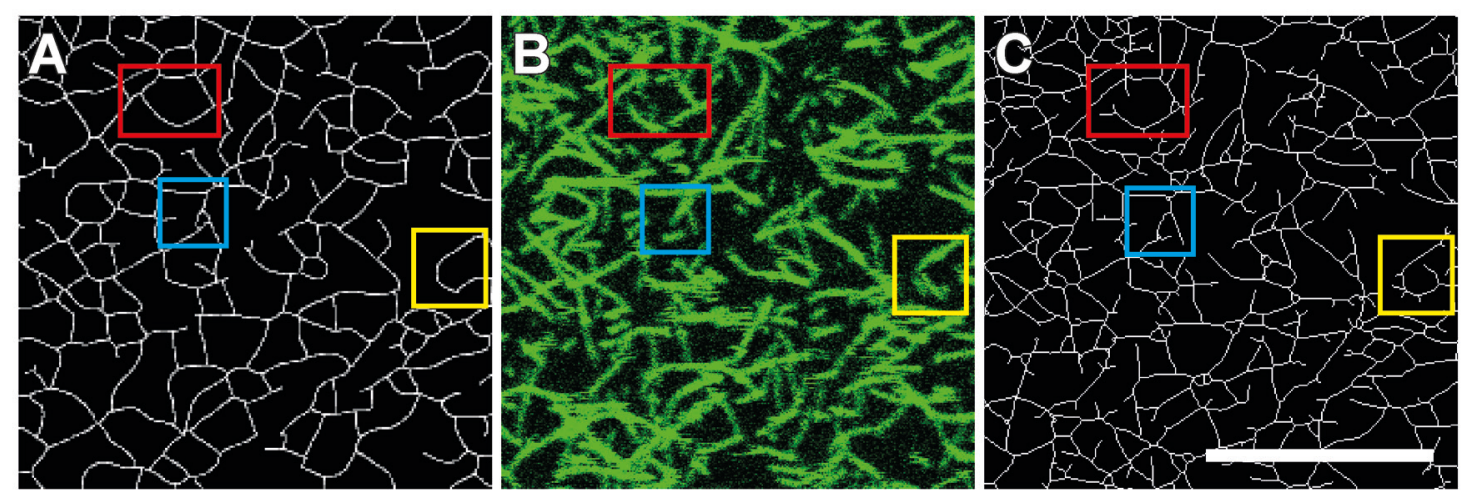

Figure 4.22: Comparison of SOAX's skeleton (A), skeleton of thresholded artificial retina (C) with the original fluorescence micrograph (B). The comparison reveals several mistakes in network skeletonization of both methods. Red box: The skeleton is bridged by the skeletonization. A small interruption (bottom) is bridged by both methods while SOAX also bridges the larger cleft on the left side. Cyan box: In this case, all interruptions are bridged by SOAX, the artificial retina bridges two of three gaps. Yellow box: In this box the branched, small filament fragments of the artificial retina's skeleton $(\mathbf{C})$ are highlighted. These fragments appear because the retina image is thresholded. Scale bar: $5 \mu \mathrm{m}$.

Bridging occurs in both skeletonization methods but seems to appear more often in case of SOAX. Furthermore, in case of SOAX skeletons, at the molar ratios of 3 and $5 \mathrm{~mol} \% \operatorname{PtdIns}[4,5] \mathrm{P}_{2}$, a dependence of image resolution on the determined node density was observed. In most cases, images with smaller resolution showed very high node densities compared to images with a higher resolution at the same pinning point density. This suggests that the bridging of the skeletons is dependent on the resolution of the image. A standardized parameter set like it was applied to this preliminary small data set is therefore not favorable for an evaluation of fluorescence images.

\section{Choice of preferred Analysis Method}

As already pointed out, a standardized routine of network skeletonization is not possible. In case of SOAX, the parameter set must be adjusted for each image and the skeleton must be compared with the original fluorescence micrograph to reduce bridging gaps and missing filaments. With regard to

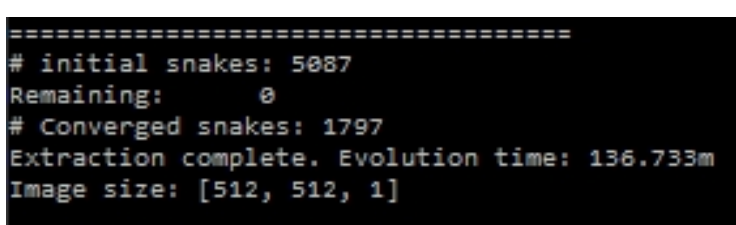

Figure 4.23: Exemplary report of SOAX. the snake evolution time, which is around 2.5 hours for a $512 \times 512$ pixel image (cf. Figure 4.23) and around 6 to 8 hours for a $1024 \times 1024$ pixel image, the evaluation of one image would require a large amount of time. On the other hand, the thresholding of the artificial retina must be checked as well. The threshold is a crucial factor to prevent or minimize filament fragmentation caused by a bad signal-to-noise ratio (cf. Figure 4.22, yellow box) as well as gap bridging. The observed differences in the 
obtained values for all four network parameters were attributed to differences in network skeletonization. In general, no skeletonization was able to show the absolute correct network skeleton as it is assumed by human eye. Both pathways of evaluation led to tendencies which can be qualitatively compared to each other to get an insight into the change of the network morphology induced by the altered pinning point density. As the artificial retina showed less mistakes by comparing the resulting skeleton with the fluorescence micrograph by naked eye (cf. Figure 4.22), the artificial retina appeared to be already a "good guess". When setting the threshold by hand and evaluating the obtained skeleton by eye, this skeletonization will be improved for the whole data set. Furthermore, a cost-benefit consideration promotes the artificial retina skeletonization. In contrast to a timescale of hours utilizing SOAX, the conversion of a fluorescence micrograph to the artificial retina with subsequent thresholding requires less than a minute.

The third reason which promotes the use of the artificial retina is the comparison with a benchmark image. As it was clearly to see that our human eye apparently works best in skeletonizing a network, two single images (2 and 5 mol \% PtdIns $[4,5] \mathrm{P}_{2}$ ) were traced by hand which served as a benchmark skeleton. The resulting values for network density parameters and filament length are presented in Table 4.4. Worth mentioning, the filament length of the SOAX skeleton was determined with the previous described method. Values output from SOAX were not considered as it was shown before that the obtained length are neither the filament length nor the segment length (cf. Figure 4.17).

Table 4.4: Exemplary comparison of SOAX's and artificial retina's skeletons with two benchmark networks traced by naked eye.

\begin{tabular}{|c|c|c|c|}
\hline network parameter & SOAX skeleton & $\begin{array}{c}\text { artificial } \\
\text { retina } \text { skeleton }\end{array}$ & $\begin{array}{c}\text { benchmark } \\
\text { skeleton }\end{array}$ \\
\hline $\begin{array}{c}\text { mesh size } \xi / \mu \mathrm{m} \\
\left(2 \mathrm{~mol} \% \text { PtdIns }[4,5] \mathrm{P}_{2}\right)\end{array}$ & $0.84 \pm 0.30$ & $0.87 \pm 0.59$ & $0.68 \pm 0.40$ \\
\hline $\begin{array}{c}\text { node density } v / \mu \mathrm{m}^{-2} \\
\left(2 \mathrm{~mol} \% \text { PtdIns }[4,5] \mathrm{P}_{2}\right)\end{array}$ & 1.18 & 1.51 & 1.48 \\
\hline $\begin{array}{l}\text { filament length } L / \mu \mathrm{m} \\
\qquad\left(2 \mathrm{~mol} \% \operatorname{Ptd} \operatorname{Ins}[4,5] \mathrm{P}_{2}\right)\end{array}$ & $4.7 \pm 3.3$ & $3.3 \pm 2.5$ & $4.9 \pm 4.0$ \\
\hline $\begin{array}{c}\text { mesh size } \xi / \mu \mathrm{m} \\
\left(5 \mathrm{~mol} \% \operatorname{PtdIns}[4,5] \mathrm{P}_{2}\right)\end{array}$ & $1.08 \pm 0.37$ & $0.82 \pm 0.46$ & $0.42 \pm 0.24$ \\
\hline $\begin{array}{c}\text { node density } v / \mu \mathrm{m}^{-2} \\
\left(5 \mathrm{~mol} \% \text { PtdIns }[4,5] \mathrm{P}_{2}\right)\end{array}$ & 1.18 & 1.53 & 2.94 \\
\hline $\begin{array}{l}\text { filament length } L / \mu \mathrm{m} \\
\quad\left(5 \mathrm{~mol} \% \operatorname{Ptd} \operatorname{Ins}[4,5] \mathrm{P}_{2}\right)\end{array}$ & $5.8 \pm 3.2$ & $3.2 \pm 5.2$ & $3.9 \pm 4.7$ \\
\hline
\end{tabular}


This benchmark comparison shows that both methods are not as good as the skeletonization by human eye. It furthermore reveals that the detected and described problems seem to increase at denser F-actin networks. The detected parameters deviate apparently more at a pinning point density generated by $5 \mathrm{~mol} \% \operatorname{PtdIns}[4,5] \mathrm{P}_{2}$ instead of $2 \mathrm{~mol} \%$. Especially comparing the mesh size $\xi$ and the node density $v$, the skeleton of the artificial retina is closer to the benchmark skeleton than SOAX. Concluding, the artificial retina is the skeletonization method of choice when making use of quality enhancement of skeletonization by checking every network of the fluorescence micrographs against the obtained skeleton. The detected values are qualitatively comparable to each other.

\section{Network Morphology in Dependence of the Pinning Point Density}

The whole data set was analyzed by skeletonization using the artificial retina. Since the change in segment length was observed to be undersized compared to the error margin, an evaluation of the segment length was renounced. This results from the heterogeneity of the network pockets which is present at all pinning point densities and is therefore no significant parameter for the change of network morphology. The heterogeneity of the networks can also be observed in case of filament length, mesh size and node density. In contrast to the segment length, no large, incomparable distribution results, but rather an overweight of small filament lengths or small meshes in contrast to larger, but rare, filaments or meshes is observed. At the node density the heterogeneity is only present as a relatively large error margin but shows almost normally distributed data. Thus, the most meaningful parameter is the node density $v$ of the resulting networks. It is not only easy to obtain but also the change induced by a varied network density is observed obviously. In order to obtain these conclusive parameters dependent on the pinning point density, a large data set was evaluated. In total, 194 fluorescence micrographs $(N=194)$ out of 22 independent preparations $(M=22)$ were evaluated as presented before. Since a complete surface coverage of ezrin T567D above 6 mol \% PtdIns[4,5] $\mathrm{P}_{2}$ was shown in chapter 4.1.3, experiments with $6 \mathrm{~mol} \%$ and $8 \mathrm{~mol} \% \quad \operatorname{PtdIns}[4,5] \mathrm{P}_{2} \quad$ were summarized within " $\geq 6 \mathrm{~mol} \%$ PtdIns $[4,5] \mathrm{P}_{2}$. . Resulting node densities are displayed in Figure 4.24. 


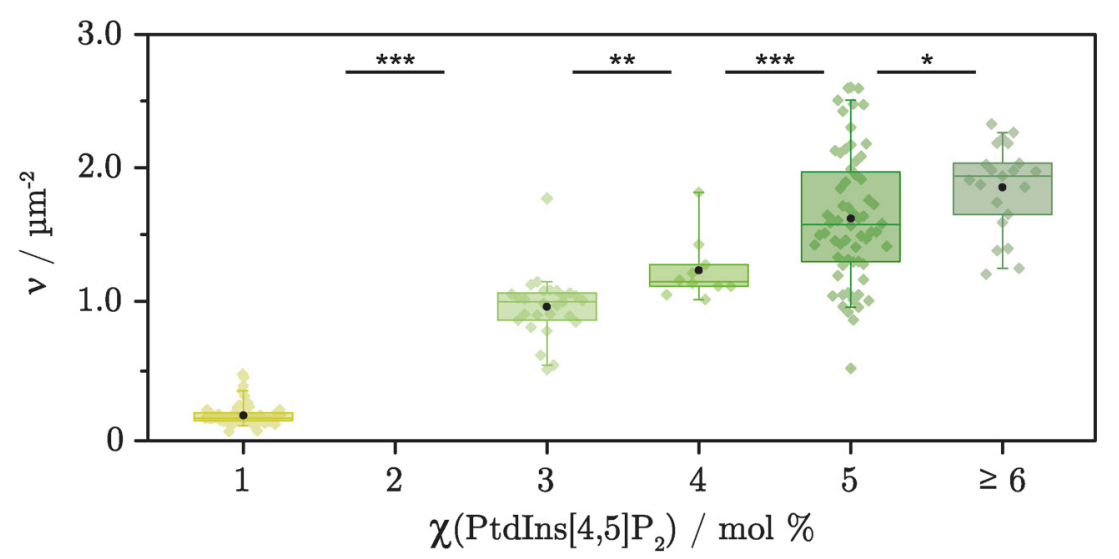

Figure 4.24: Node density $v$ of membrane-attached F-actin networks as a function of the pinning point density. ${ }^{[160]}$ Pinning point density is described as the relating PtdIns $[4,5] \mathrm{P}_{2}$ concentration in the lipid bilayer. Node densities of networks anchored to lipid bilayers with $1 \mathrm{~mol} \%(N=63, M=3), 3 \mathrm{~mol} \%(N=27, M=6), 4 \mathrm{~mol} \%(N=10, M=2)$, $5 \mathrm{~mol} \%(N=64, M=7)$ and over $6 \mathrm{~mol} \% \operatorname{PtdIns}[4,5] \mathrm{P}_{2}(N=30, M=4)$ are displayed. With increasing pinning point density, a clear tendency to higher node densities is observed. Box plots extent from $25^{\text {th }}$ to $75^{\text {th }}$ percentile and whiskers from $5^{\text {th }}$ to $95^{\text {th }}$. A Mann-Whitney $U$ test was performed to show that two populations are not populations with equal means. Significance levels are: $5 \%(*), 1 \%(* *)$ and $0.1 \%(* * *)$. Modified and reprinted with permission from Nöding, Schön et al. Copyright 2018 American Chemical Society. ${ }_{[160]}$

The respective node density reveals a clear tendency to more nodes per square micron indicating a denser network at higher pinning point densities. More PtdIns $[4,5] \mathrm{P}_{2}$ within the lipid bilayer and therefore more attachment points for the F-actin network leads to more compact MACs. Node densities above $\chi_{\operatorname{PtdIns}[4,5] \mathrm{P}_{2}}=6 \mathrm{~mol} \%$ were identically and therefore summarized as mentioned before. Therefore, relation between node density and ezrin surface coverage is clearly consistent. In contrast to networks attached to membranes with $\chi_{\operatorname{PtdIns}[4,5] \mathrm{P}_{2}}=1 \mathrm{~mol} \%\left(v=(0.12 \pm 0.03) \mu \mathrm{m}^{-2}\right)$, a 16-fold increase was observed on membranes with $\chi_{\operatorname{PtdIns}[4,5] \mathrm{P}_{2}} \geq 6 \mathrm{~mol} \%(v=(1.94 \pm$ 0.46) $\mu^{-2}$ ). While the ezrin T567D surface coverage showed a sigmoidal increase in surface coverage, the dependence in node density appears to be almost linearly as $v \propto\left(\chi_{\mathrm{PtdIns}[4,5] \mathrm{P}_{2}}\right)^{1.1}$. Already at membranes with $\chi_{\mathrm{PtdIns}[4,5] \mathrm{P}_{2}}=3 \mathrm{~mol} \%(v=(1.00 \pm$ $0.11) \mathrm{\mu m}^{-2}$ ), an 8 -fold increase was observed.

The second parameter for MAC density indication is the mesh size. As already mentioned, the mesh size parameter is not as reliable as the node density due to different geometries and an excess weight of small meshes across the whole F-actin network. Due to that fact, the resulting meshes were further weighted by their area. The detected mesh sizes (Figure 4.25 A) as well as a cumulative plot (Figure $4.25 \mathrm{~B}$ ) as a function of the pinning point density are shown in Figure 4.25. 

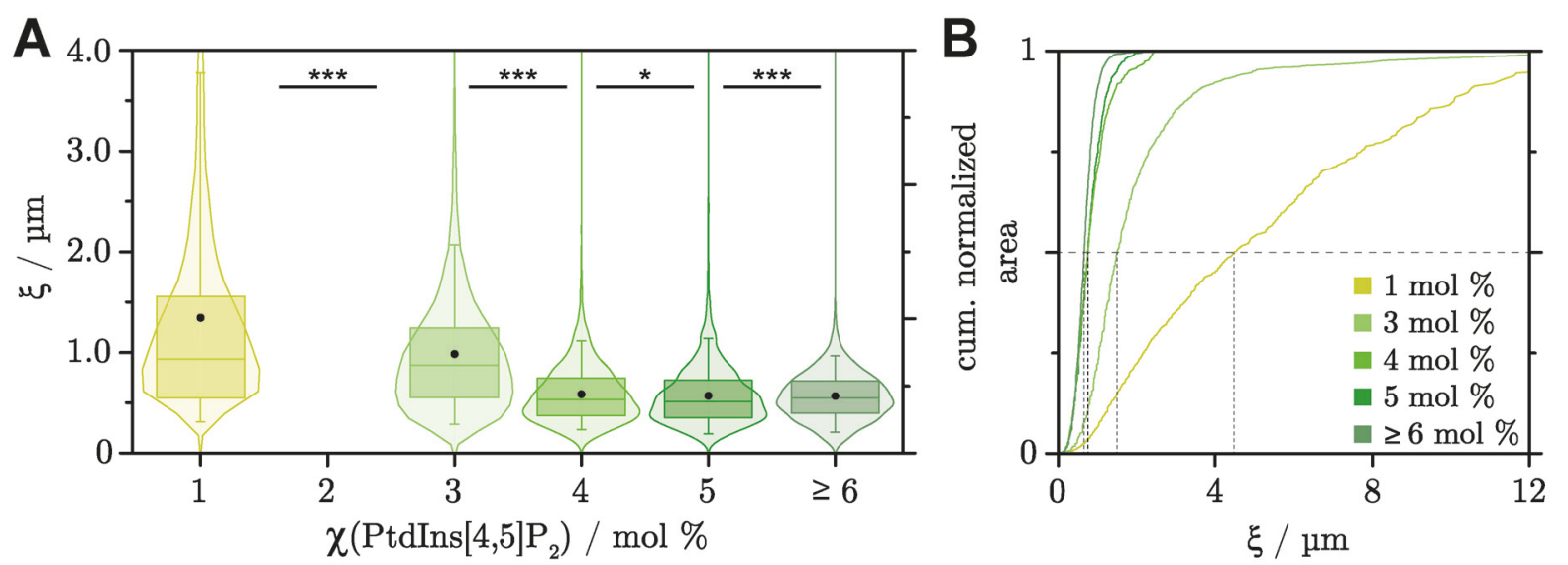

Figure 4.25: Mesh size $\xi$ of membrane-bound F-actin networks as a function of pinning point density represented as relating $\operatorname{PtdIns}[4,5] \mathbf{P}_{2}$ content. A: Violin plots display the obtained mesh size with relating density function. From $1 \mathrm{~mol} \%$ to $3 \mathrm{~mol} \%$ a clear decrease in mesh size is observed, while above $4 \mathrm{~mol} \%$ no further obvious change is detected. Box plots extent from $25^{\text {th }}$ to $75^{\text {th }}$ percentile and whiskers from $5^{\text {th }}$ to $95^{\text {th }}$. A Mann-Whitney $U$ test was performed to show that two populations are not populations with equal means. Significance levels are: $5 \%\left({ }^{*}\right), 1 \%\left({ }^{* *}\right)$ and $0.1 \%(* *)$. B: A cumulative plot of meshes normalized with the area of the circle shows the tendency more clearly. Mean values respectively for every pinning point density were recorded at a cumulative normalized area of 0.5 (dotted lines).

Considering the regime with a low pinning point density (1-3 mol \% PtdIns $\left.[4,5] \mathrm{P}_{2}\right)$, the detected change in mesh size shows a clear tendency to significantly smaller mesh sizes with increasing pinning point density. This tendency is strongly attenuated or undetectable in the regime of high pinning point densities $\left(\geq 4 \mathrm{~mol} \% \operatorname{Ptd} \operatorname{Ins}[4,5] \mathrm{P}_{2}\right)$. Furthermore, the bodies of the violins display the density function of obtained mesh sizes which demonstrates the mentioned excess weight of smaller meshes, because the vertical plotted functions are mostly left-skewed with the maximum below the mean and median value. A broad shoulder to higher mesh sizes completes this representation. This dependency can be illustrated more clearly by weighting the resulting mesh size diameter with the area of the circle. Big meshes occur rarely but represent a large area of the image. Therefore, the obtained value of one big mesh is large, but suppressed in the statistics by the large frequency of occurrence of small meshes and weighting leads to the fact that this value is taken more into account. A cumulative plot (cf. Figure $4.25 \mathrm{~B}$ ) is used to determine the mean values of the resulting weighted mesh size distribution. The obtained mean values are illustrated as dotted lines intersecting the plots at cumulative normalized area of 0.5. Resulting mean values are summarized and compared to unweighted mean values in Table 4.5. 
Table 4.5: Obtained mesh sizes $\xi$ compared with mesh sizes weighted by the area of the detected circles.

\begin{tabular}{c|c|c|c|c|c}
$\begin{array}{c}\text { PtdIns[4,5]P } \\
\text { content }\end{array}$ & $\mathbf{1}$ mol \% & $\mathbf{3}$ mol \% & $\mathbf{4}$ mol \% & $\mathbf{5}$ mol \% & $\mathbf{2} \mathbf{6} \mathbf{~ m o l ~ \%}$ \\
\hline $\begin{array}{c}\text { mesh size } / \\
\mathbf{\mu m}\end{array}$ & $1.34 \pm 1.41$ & $0.98 \pm 0.63$ & $0.58 \pm 0.28$ & $0.57 \pm 0.30$ & $0.57 \pm 0.23$ \\
$\begin{array}{c}\text { unweighted } \\
\text { mesh size } / \\
\mathbf{\mu m} \\
\text { weighted }\end{array}$ & 4.54 & 1.56 & 0.82 & 0.82 & 0.72
\end{tabular}

In general, the tendency to smaller mesh sizes with increasing pinning point density is observed in unweighted as well as weighted data. At higher pinning point densities (above $4 \mathrm{~mol} \%$ PtdIns $[4,5] \mathrm{P}_{2}$ ) just small mesh size changes appear or they are observable as a shrinking of the error margin (from 5 to $\geq 6 \mathrm{~mol} \%$ ). The weighted data shows that the change between 4 and $5 \mathrm{~mol} \%$ is smaller than the ongoing change to $6 \mathrm{~mol} \%$. It is further shown that very big meshes apparently occur below $3 \mathrm{~mol} \%$, since the mean mesh sizes of the weighted data approaches closer to the mean mesh size of the unweighted date with increasing pinning point density.

Last evaluated network parameter is the filament contour length $L$. All lengths were collected from 194 images and the resulting frequency distribution was observed as a strongly left-skewed histogram (not shown for clarity). As done before for filament lengths in 3D F-actin gels, a log-normal function (cf. equation 4.2) was fitted to the data to determine the filament length. Resulting parameters are displayed in Table 4.6.

Table 4.6: Detected filament lengths $(L)$ utilizing a log-normal fit of the left-skewed length distribution. Mean value, geometric mean value (synonymous to actuarial expectation), standard deviation and confidence interval are given in detail.

\begin{tabular}{c|c|c|c|c}
$\begin{array}{c}\text { PtdIns[4,5]P } \\
\text { content }\end{array}$ & $\begin{array}{c}\text { mean } \\
\text { value } \\
\boldsymbol{\mu} / \mathbf{\mu m}\end{array}$ & $\begin{array}{c}\text { geometric } \\
\text { mean } \\
\text { value } \\
\boldsymbol{\mu}^{*} / \mathbf{\mu m}\end{array}$ & $\begin{array}{c}\text { standard } \\
\text { deviation } \\
\boldsymbol{\sigma} / \mathbf{\mu m}\end{array}$ & $\begin{array}{c}\text { confidence interval (68.3 \%) } \\
{\left[\boldsymbol{\mu}^{*} / \boldsymbol{\sigma}^{*}, \boldsymbol{\mu}^{*} \cdot \boldsymbol{\sigma}^{*}\right] / \boldsymbol{\mu m}}\end{array}$ \\
\hline $\mathbf{1} \mathbf{~ m o l ~ \%}$ & 1.55 & 4.71 & 1.09 & {$[1.58,14.04]$} \\
$\mathbf{3} \mathbf{~ m o l ~ \%}$ & 1.22 & 3.38 & 1.21 & {$[1.01,11.30]$} \\
$\mathbf{4} \mathbf{~ m o l ~ \%}$ & 1.14 & 3.12 & 1.22 & {$[0.92,10.61]$} \\
$\mathbf{5} \mathbf{~ m o l ~ \%}$ & 1.09 & 2.97 & 1.36 & {$[0.76,11.58]$} \\
$\mathbf{2} \mathbf{6 ~ m o l ~ \%}$ & 1.00 & 2.71 & 1.21 & {$[0.81,9.09]$} \\
\hline
\end{tabular}


Already the mean value, but more specifically the geometric mean value, shows a significant tendency to smaller filament lengths with increasing pinning point density. The detected change is slight but consistent. Also, the confidence interval, which includes a probability of $68.3 \%$ of all detected lengths, shows a smaller range with increasing pinning points and respectively more binding sites for attachment of the F-actin network. This fact is quite surprising because the network before attachment is pre-polymerized with a certain filament length. This detected filament length in pre-polymerized 3D networks is larger than the observed filament length in MACs. Therefore, the attachment process of the F-actin network must induce some kind of sorting effect. Interestingly, the sorting effect seems to be stronger when more binding sites for F-actin are present as the detected filament length in MACs decreases with increasing pinning point density. One possibility is that only short filaments attach to the ezrin T567D decorated lipid bilayer. This would imply that all detected long bundled filaments observed in microfluidic channels or chambers are sorted out and are rinsed away because they had not been attached to the $C$-ERMAD of ezrin T567D. Contrary to this assumption, no longer filaments than the observed and recorded ones were detected before rinsing. A further possibility is that the long pre-polymerized filaments are breaking or rupture during the attachment process to the $C$-ERMAD of ezrin. This would explain why the long filaments are not observed laying on the MAC before rinsing the specimen. 


\subsubsection{Effect of MAC attachment to the mobility of lipid bilayers}

Using fluorescence recovery after photo bleaching experiments (FRAP, chapter 3.5.2.2) fluidity of the lipid bilayer was determined. Therefore, time series of fluorescence micrographs of solid supported lipid bilayers doped with 0.4 mol \% TexasRed-DHPE were taken in an interval of around $300 \mathrm{~ms}$. Three images were taken before the bleach was applied (Figure 4.26 A, $t=-1 \mathrm{~s}$ ). Various lipid bilayers containing either $3 \mathrm{~mol} \%$ or $5 \mathrm{~mol} \% \mathrm{PtdIns}[4,5] \mathrm{P}_{2}$ were used. As diffusion coefficients of both lipid compositions with and without MAC were basically identical, measurements were cumulated to obtain one diffusion coefficient without and one with MAC, respectively.
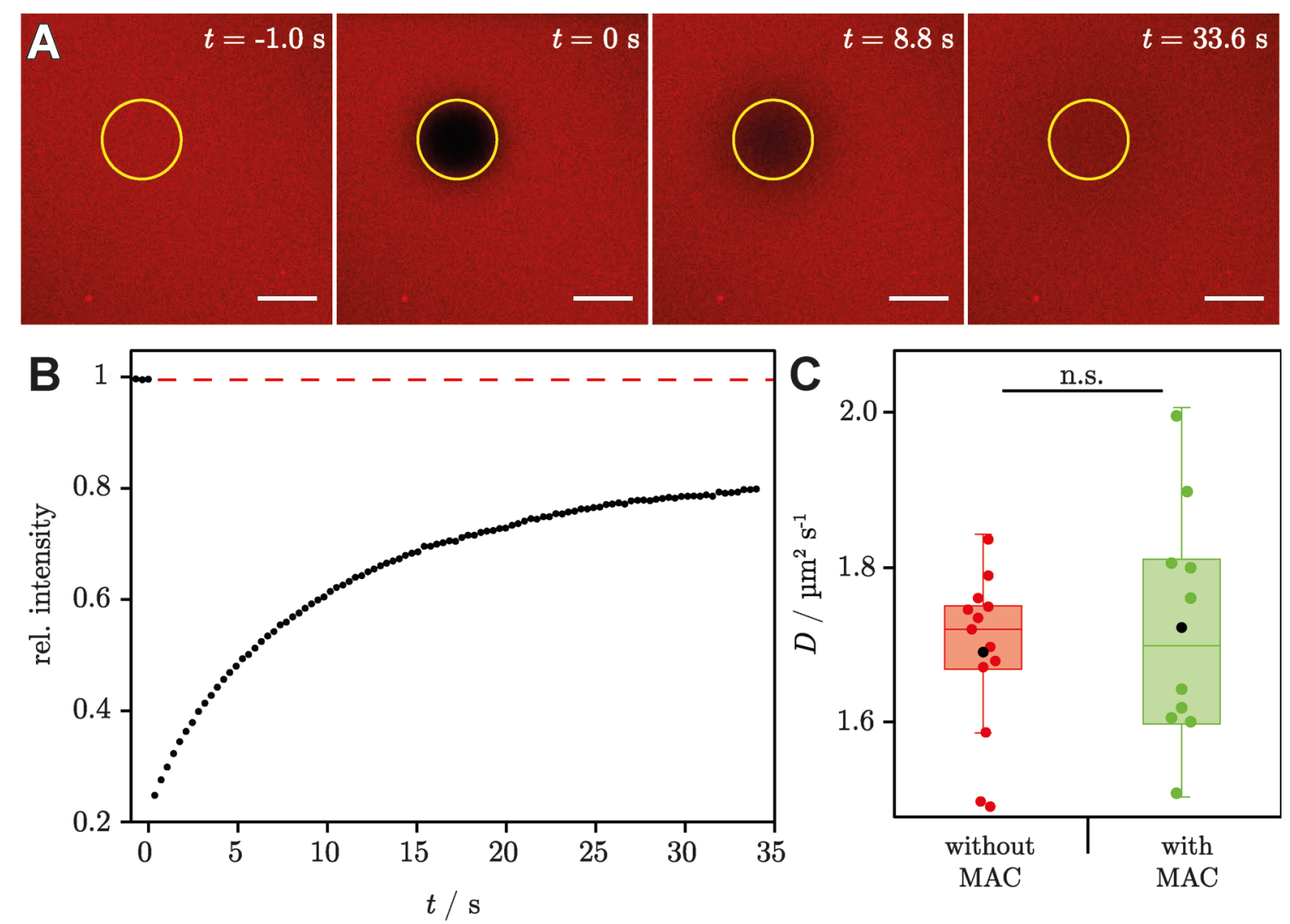

Figure 4.26: Exemplary FRAP experiment starting with a time series of fluorescence micrographs. ${ }^{[160]}$ A: Fluorescence intensity of TxR-DHPE is recorded in a time resolved manner to track bleaching $(t=0 \mathrm{~s})$ and subsequent recovery $(t=8.8 \mathrm{~s}$ and $t=33.6 \mathrm{~s}$ ) in a region of interest (ROI, yellow). Scale bars: $10 \mu \mathrm{m}$. B: Relative fluorescence intensity is recorded for every ROI within the time series. Normalized and bleaching corrected data is plotted and evaluated as described in literature. ${ }^{[159]}$ C: Box plot diagram displaying determined diffusion coefficients of $(1.7 \pm 0.1) \mu^{2} \cdot \mathrm{s}^{-1}(N=13)$ for lipid bilayers without MAC and $(1.7 \pm 0.2) \mathrm{\mu m}^{2} \cdot \mathrm{s}^{-1}(N=10)$ for lipid bilayers with MAC. Box plots extent from $25^{\text {th }}$ to $75^{\text {th }}$ percentile and whiskers from $5^{\text {th }}$ to $95^{\text {th }}$. Modified and reprinted with permission from Nöding, Schön et al. Copyright 2018 American Chemical Society.[160]

At time point zero (Figure $4.26 \mathrm{~A}, t=0 \mathrm{~s}$ ), the fluorescence intensity was bleached by a strong laser pulse $(\lambda=561 \mathrm{~nm})$ in a region of interest (Figure $4.26 \mathrm{~A}$, yellow circle) 
and the subsequent recovery (Figure $4.26 \mathrm{~A}, t=8.8 \mathrm{~s}$ and $t=33.6 \mathrm{~s}$ ) was detected. The mean intensity was corrected against general bleaching by taking any spot beyond the ROI into account. Normalized data (Figure $4.26 \mathrm{~B}$ ) was used to determine the diffusion coefficient by a MATLAB based program from Jönsson et al.[159] Evaluation revealed no significant difference between lipid bilayers without any attachment $\left(D=(1.7 \pm 0.1) \mu^{2} \cdot \mathrm{s}^{-1}\right)$ and lipid bilayers with an attached minimal actin cortex $\left(D=(1.7 \pm 0.2) \mathrm{\mu m}^{2} \cdot \mathrm{s}^{-1}\right.$, Figure $\left.4.26 \mathrm{C}\right)$. The detected diffusion coefficients are in a good accordance with values found in literature. ${ }^{[119,159]}$ Braunger et al. showed a diffusion coefficient of $D=(2.9 \pm 0.2) \mathrm{um}^{2} \cdot \mathrm{s}^{-1}$ for a solid supported lipid bilayer consisting of POPC/PtdIns[4,5] $\mathrm{P}_{2}$ /bodipy-TMR-PtdIns[4,5] $\mathrm{P}_{2} /$ perylene (96:2.5:0.5:1) which is slightly higher than the diffusion coefficient of this work. Several differences can explain the slight divergence. On the one hand, they analyzed the diffusion coefficient by bleaching labeled PtdIns $[4,5] \mathrm{P}_{2}$ lipids. The fluorophore bodipy-TMR is connected to one fatty acid which could lead do a change in lateral mobility of the lipid bilayer. On the other hand, their substrate functionalization was nearly the same but with an additional step. The hydrophilic surface functionalization was performed with a final treatment with oxygen plasma. Oxygen plasma is known to increase the hydrophilicity of a surface which was verified by a decreasing contact angle of a water droplet. ${ }^{[199]}$ A higher hydrophilicity could therefore explain a higher lateral mobility which would be consistent between this work and the work of Braunger et al. Furthermore, they showed a decreased mobility of PtdIns $[4,5] \mathrm{P}_{2}$ by factor 3 on a supported hybrid membrane (SHM) when ezrin is bound to PtdIns $[4,5] \mathrm{P}_{2}$. These SHMs consist of a dodecyltrichlorosilane-functionalized substrate with only a lipid monolayer on top. It seems reasonable that ezrin bound PtdIns $[4,5] \mathrm{P}_{2}$ diffuses slower than unbound lipid. Since a SHM only consists of a monolayer, and only accessible receptor lipids will be affected by protein binding, this effect is softened in system utilizing a lipid bilayer because one leaflet remains totally unaffected. Considering the lateral mobility of the total lipid bilayer, the decrease of lateral lipid mobility should also be attenuated because only the receptor lipid, present in a low ratio, is affected. The experiments in this work indicates that this decrease of $\operatorname{PtdIns}[4,5] \mathrm{P}_{2}$ mobility vanishes within the lateral mobility of the whole lipid bilayer which is analyzed by bleaching TxR-DHPE. Therefore, the ezrin binding and subsequent attachment of an F-actin network does not alter the fluidity of the whole lipid bilayer but will decrease the mobility of the receptor lipid. The nonexistent difference between diffusion coefficients with different ratios of $\mathrm{PtdIns}[4,5] \mathrm{P}_{2}$ supports this assumption.

In contrast to these findings an article about "lateral membrane diffusion modulated by a minimal actin cortex" is found in literature. ${ }^{[200]}$ They showed that an F-actin network attached to pore spanning lipid bilayers $(\varnothing=2.5 \mu \mathrm{m})$ via a neutravidin/biotin-tagged lipid can decrease the lipid mobility from $D=(9.9 \pm 0.6) \mathrm{\mu m}^{2} \cdot \mathrm{s}^{-1}$ to 
$D=(4.8 \pm 0.4) \mu^{2} \cdot \mathrm{s}^{-1}$. The generally higher diffusion coefficients can be explained by the fact that these were measured on a free-standing lipid bilayer instead of a solid supported membrane as used in this work. Diffusion coefficients were determined by means of fluorescence correlation spectroscopy (FCS). FCS was measured either using the Alexa Fluor ${ }^{\circledR} 647$ labeled protein CtxB or the lipid bound fluorophore Atto $647 \mathrm{~N}$ DOPE. By determining the actin density with averaging the fluorescence intensity in the area of FCS measurement, they correlated a decreased lipid mobility with an increasing density of the actin network. Interestingly, the diffusion coefficient of the protein-bound fluorophore showed a stronger decrease than coefficient of the fluorescent lipid. This indicates that a direct interaction of the diffusive species, the protein or the lipid-bound fluorophore head, with the actin network also has a strong effect. Additionally, the change of anchor points for the F-actin network by decreasing the ratio of used biotinylated actin from $20 \%$ to $5 \%$ did not result in any change of the lipid mobility. This supports the hypothesis of this work that anchoring the MAC is not a major source of mobility reduction. Rather the collision or interaction of the bulky protein or the fluorophore with the actin filaments appears to be the main reason. This shows that the results and accordingly this work are not contrary with the work of Heinemann et al. [200]

In total, this work and the literature show that anchoring an F-actin network to a lipid bilayer does not modulate the lateral mobility of the underlying membrane. Of course, ezrin bound receptor lipids $\left(\operatorname{Ptd} \operatorname{Ins}[4,5] \mathrm{P}_{2}\right)$ are hindered in their mobility as shown by Braunger et al. ${ }^{[119]}$ but the overall mobility is not changed significantly. However, it was already shown that a natural cell cortex underneath the plasma membrane is able to reduce the mobility of the plasma membrane.[201,202] The decrease in mobility presented in this study of Iino et al. was strongly dependent on the oligomer size of the membrane protein E-cadherin which led to the clue that the interaction of E-cadherin and the actin skeleton is mainly responsible for the decrease in diffusivity. ${ }^{[202]}$ Concluding, further agents like proteins or membrane interacting, actin binding proteins must be the reason how the cell cortex can have an effect on the lateral membrane mobility.

\subsubsection{Discussion of Self-Organization of F-actin Attachment to Artificial Lipid Bilayers}

It was presented that the specific attachment of F-actin networks to an ezrin-covered lipid bilayer, to form minimal actin cortices, is highly dependent on the pinning point density. The F-actin network was shown to be a flat layer on the membrane, which is organized differently at various ratios of $\operatorname{PtdIns}[4,5] \mathrm{P}_{2}$, resulting in various network 
morphologies. These morphological changes were accompanied with a tendency to denser networks when increasing the pinning point ratio. The evaluation of the network morphology was found to be highly challenging as the skeletonization of fluorescence micrographs of MACs was difficult to implement, because of different image resolutions and signal-to-noise ratios. Different image resolutions can hardly be avoided as magnifications improve the detection of network meshes and node densities but restrict the resulting detectable filament length. The comparison of the resulting skeletons of the software SOAXs and thresholded retina images with a benchmark showed advantages using the artificial retina but also that no computational skeletonization is as good as the human eye. Evaluation of a large data set of fluorescence micrographs recorded on MACs, using pinning point densities between 1 and $8 \mathrm{~mol} \% \operatorname{Ptd} \operatorname{Ins}[4,5] \mathrm{P}_{2}$, confirmed the increasing density of the F-actin network at ascending pinning point density. A rise in the node density and a decrease in the mesh size of the resulting networks with increasing pinning point densities was observed. Furthermore, shorter filament contour lengths were found at higher node densities. Interestingly, the contour length obtained at MACs was smaller than the contour length obtained in 3D F-actin gels. While SOAX revealed a filament length of $(17 \pm 3) \mu \mathrm{m}$ in $3 \mathrm{D}$ actin gels, the filament length at MACs was determined to be around 1 um (from artificial retina analysis). In general, it was shown that SOAX detects longer filament lengths, thus no clear shortening factor can be given. In order to obtain a comparable value, the artificial retina was applied to $2 \mathrm{D}$ projections of $3 \mathrm{D}$ F-actin network's $z$-scans, which were achieved by averaging the intensity in z-direction, and the resulting skeleton was evaluated. The filament length was determined to $(5.6 \pm 3.3) \mu \mathrm{m}$ which is still significantly higher than the observed filament length in MACs. The software SOAX works better in 3D because the program was originally developed to obtain information about biopolymer networks in 3D.[162,163,195,203] The artificial retina was shown to perform better in 2D (cf. chapter 4.2.3) but transferring a 3D image into 2D rejects spatial information which SOAX can use. Therefore, it is assumed that the detected 3D filament length of SOAX is more reliable but hard to compare to the obtained values in 2D. To sum up, the factor of filament shortening, when attaching an F-actin network to an ezrin-decorated lipid bilayer, is still unclear but is in a range of 5 to 15 -fold shorter. Filament lengths of MACs are only rarely presented in literature, but Vogel et al. showed myofilament lengths (actomyosin-networks) of about $600 \mathrm{~nm}$ using topographical AFM images and using a biotin-neutravidin linkage. ${ }^{[82]}$ These myofilaments are slightly shorter than filaments out of this work, but they also detected a decreasing filament length with increasing F-actin density. Due to the fact that they did not quantified the F-actin density, no further comparison is possible. A comparable quantitative parameter for the density of F-actin networks was reported by Morone et al., who determined mesh sizes of NRK (normal rat kidney fibroblast) and FRSK (fetal 
rat skin keratinocyte) cell fragments by means of electron microscopy. ${ }^{[44]}$ They showed mesh sizes of 50 or $200 \mathrm{~nm}$ dependent on the cellular system. On the one hand, this demonstrates that the mesh size of an actin network is highly dependent on the cell type, but it also shows that mesh sizes observed in this work are in a similar range concerning high ratios of PtdIns $[4,5] \mathrm{P}_{2}$. It has to be considered that the membrane skeleton of Morone et al. presents a top-down approach, therefore an cross-linked network in contrast to the presented minimal actin cortex of this work. This suggests, that cross-links, which are present in NRK or FRSK cell fragments, may lead to an increasing of F-actin network density. This tendency would also be highly dependent on the present type of cross-linker, which could explain why FRSK cells show a mesh size of $50 \mathrm{~nm}$ while NRK cells show a less dense cortex with a mesh size of around $200 \mathrm{~nm} \cdot{ }^{[44]}$

At the attachment process of the presented MAC, a sorting-effect arises which is assumed to be a breaking of filaments more likely than a binding effect of only short polymerized filament. This hypothesis is supported by the fact that the observed long and thick filament are never present on the substrate, even not before rinsing the specimen after the F-actin network is incubated on the lipid bilayer. The occurring remodeling during the attachment progress is surprisingly fast as it was not possible to image this process by means of CLSM.

The observed filament length of in vitro pre-polymerized F-actin network gels showed the usually reported length of about 10-20 $\mu \mathrm{m},{ }^{1197,204]}$ while in living cells the filaments are substantially shorter with contour lengths smaller than $2 \mu \mathrm{m} .{ }^{[205]}$ The experiments in this work indicate that not only the presence of many actin binding proteins or the treadmilling effect of $\mathrm{F}$-actin reduces the filament length of the natural cell cortices but also the attachment of the cell cortex to the plasma membrane is a major impact for the shorter actin filament lengths and the resulting network architecture. A possible origin for that is given by Chugh et al. who showed that filament shortening is a promising way to modulate the plasma membrane tension. ${ }^{[43]}$ Filament breakage and rupture within in vivo processes is believed to be mostly induced by active cortex components like myosin motor proteins. ${ }^{[5]}$ The tensile strength of phalloidin-stabled, single actin filaments was found to be independent from filament length and was determined to $F=(108 \pm 5) \mathrm{pN}$ by means of micromanipulation experiments. ${ }^{[206]}$ The breakage induced by myosin II was already shown in an in vitro approach within a formed MAC. ${ }^{[82]}$ Remarkably, many articles in literature focus on the morphologic change as a result of the motor protein's action but the experiments in this work show that the architecture of the MAC is also very important for network organization. No active components, like myosin II motor proteins, were used and the sorting effect, which appears during MAC formation, can only be induced by providing attachment points and the binding process of ezrin to F-actin. The change in pining point density 
can regulate the density of the resulting MAC which underlines the importance of the organization and composition of the underlying plasma membrane. Recent studies showed how the self-organization of the cytoskeleton is able to generate distinct patterns like stars or asters which are primarily driven by the nucleation of the Arp2/3 complex.[207,208] Concluding, the presented experiments revealed insights in the importance of F-actin network attachment and the relating pinning point density. The process of attachment itself appears to be a great rearrangement which is not understood yet. Diverse observations in literature could be supported and a clear correlation between the network density and the pinning point ratio was made. Furthermore, it was shown that the attachment of MACs has no significant impact on the mobility of the underlying lipid bilayer.

The importance of the self-organization is mainly associated with an influence on the mechanical properties of the cell which is controversial discussed in literature. ${ }^{[43,207]}$ The presented MAC and the observed changes in the morphologic structure will be used to characterize the impact on the F-actin network mechanics in the following section. 


\subsection{Mechanics of F-actin Networks}

\subsubsection{Frequency-Dependent Viscoelastic Properties of F-actin Networks depending on Pinning Point Density}

- Some experiments in this chapter have been published in "Rheology of MembraneAttached Minimal Actin Cortices" by H. Nöding, M. Schön, C. Reinermann, N. Dörrer, A. Kürschner, B. Geil, I. Mey, C. Heussinger, A. Janshoff and C. Steinem, J. Phys. Chem. B, 2018, 122, 4537-4545 [160]-

- Microrheology experiments were performed in cooperation with Helen Nöding as part of project A17 of the collaborative research center $937^{[166]}$

In a next step the mechanical properties of the obtained MACs (cf. chapter 4.2) were investigated. For this, the viscoelastic properties of MACs were evaluated by means of video particle tracking which is a passive form of microrheology (cf. chapter 3.6.4). The effect of membrane attachment as well as the adjustment of network density obtained in the previous chapter is investigated. As tracer particles carboxylate-modified polystyrene beads with a radius of $1 \mu \mathrm{m}$ were chosen, large enough to achieve a suitable signal-to-noise ratio and small enough to fit into the networks meshes and cortex height. It was found that carboxylate-modified polystyrene beads bind non-specifically but provide a stable interaction with a polymer network. ${ }^{[209]}$ In a broad frequency regime as displayed in chapter 3.6.4 the shear modulus $G^{*}(f)=G^{\prime}(f)+G^{\prime \prime}(f)$ of the respective MAC has been made accessible with the technique of VPT.[168] Frequency dependent viscoelastic properties are mostly collected in 3D F-actin network gels and only rarely reported in membrane-attached systems. Therefore, rheological data of 3D F-actin was collected in our setup and subsequently the data of an F-actin network attached to a lipid bilayer were recorded to determine the influence of attachment itself and the influence of ezrin T567D binding.
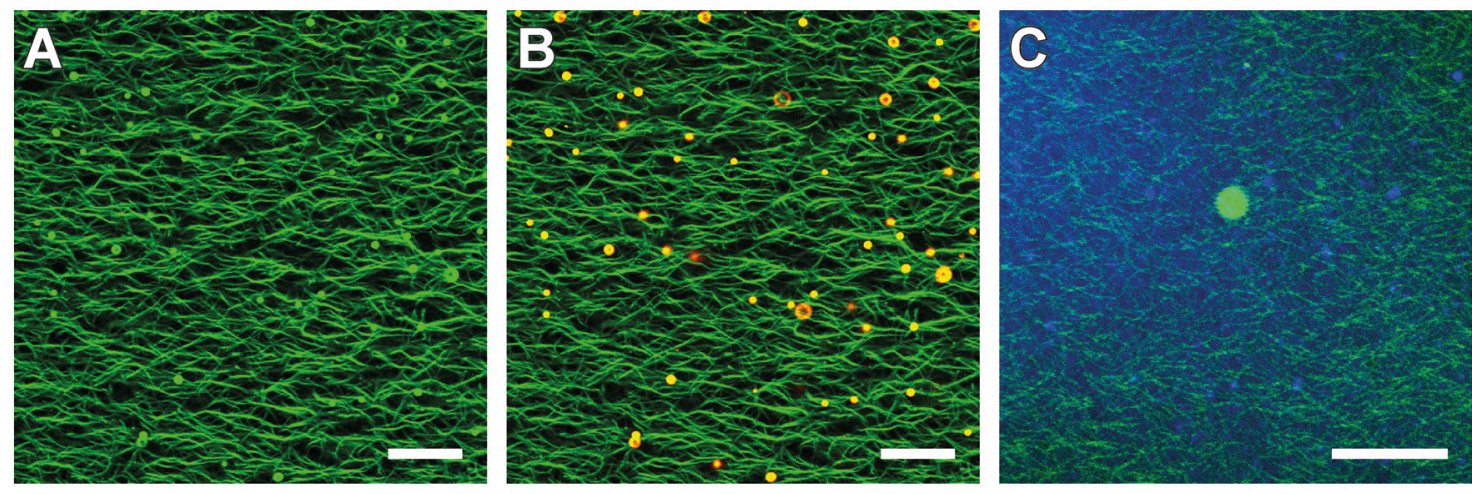

Figure 4.27: Polymerized F-actin networks containing beads with a radius of $1 \mathrm{\mu m}$. A/B: A fluorescence micrograph of polymerized F-actin networks labeled with 
Alexa Fluor ${ }^{\circledR}$ 488-phalloidin in a microfluidic channel is shown. The red fluorescence of the beads cross-talks into the detected green fluorescence, therefore an overlay of both signals is shown in (B). C: Fluorescence micrograph of an MAC containing a single bead integrated in an F-actin network bound to a lipid bilayer consisting of POPC/PtdIns[4,5] $\mathrm{P}_{2} /$ Atto 390-DOPE (96:3:1). Again, the bead is clearly visible in green fluorescence due to cross-talk. Scale bars: A/B: $50 \mu \mathrm{m}$. C: $15 \mu \mathrm{m}$.

The preparation procedure for MACs remained nearly the same, the only difference was the presence of tracer particles (beads) during polymerization of the network. In Figure 4.27 an F-actin network with beads in a microfluidic channel (A/B) and an MAC containing a single bead (C) is shown. Since the bead fluorescence cross-talks into the green fluorescence obtained from Alexa Fluor ${ }^{\circledR}$ 488-phalloidin, the tracer particles incorporation into the F-actin network was characterized further. For this reason, non-fluorescent tracer particles with the size of $2 \mu \mathrm{m}$ in diameter were added during polymerization as it was done for fluorescent tracer particles. An F-actin network containing non-fluorescent beads was attached to a lipid bilayer containing $3 \mathrm{~mol} \% \mathrm{PtdIns}[4,5] \mathrm{P}_{2}$ via ezrin T567D binding. The obtained network morphology is characterized using fluorescence micrographs shown in Figure 4.28.
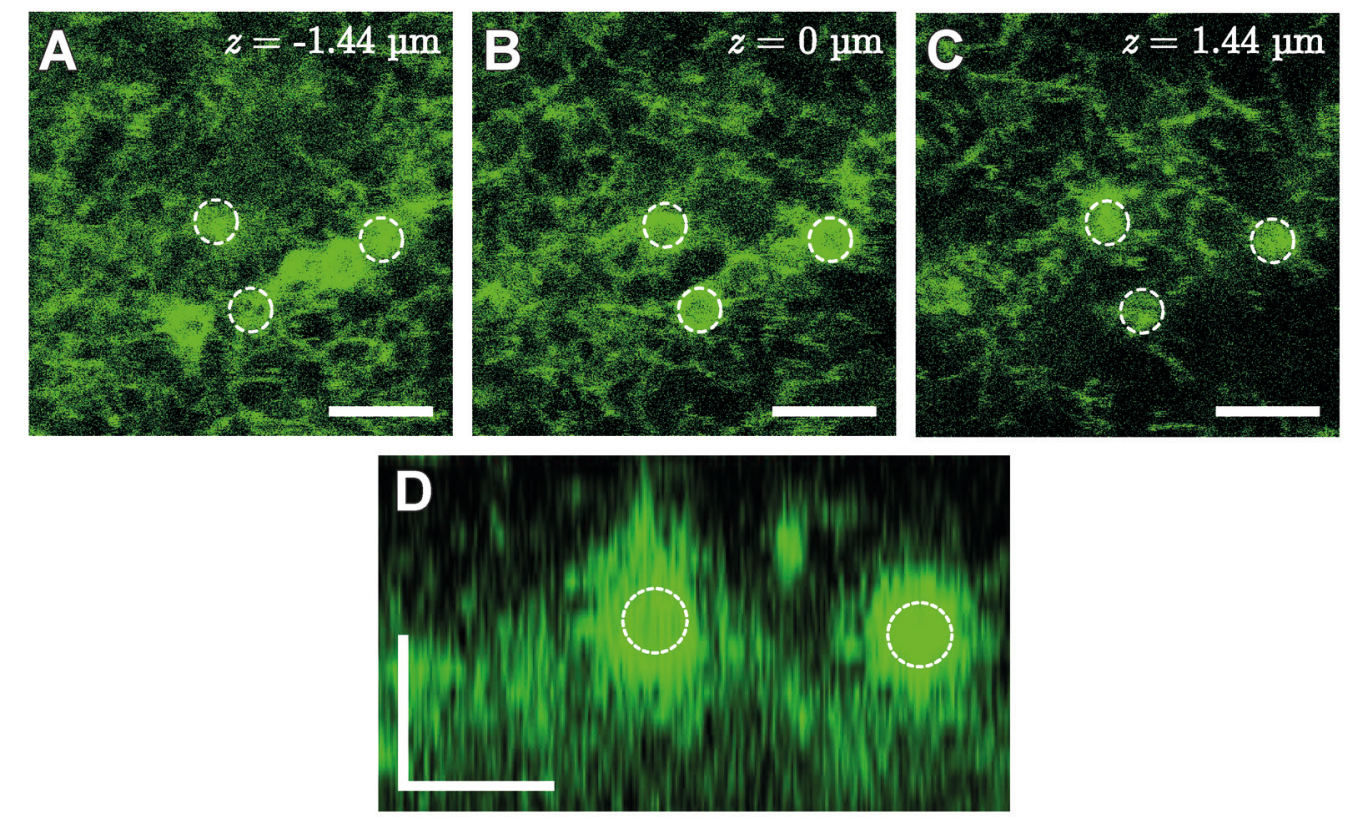

Figure 4.28: Fluorescence micrographs visualize the incorporation of beads into the MAC. An F-actin network labeled with Alexa Fluor ${ }^{\circledR}$ 488-phalloidin with non-fluorescent beads was attached ezrin-mediated to a lipid bilayer containing 3 mol \% PtdIns $[4,5] \mathbf{P}_{2} \cdot{ }^{[160]}$ A-C: Three images out of one $z$-stack which show three incorporated beads. As the beads themselves are non-fluorescent their estimated position localized via actin accumulation around the beads is illustrated within the graphic (white dotted circles). D: An orthogonal view shows that at local bead positions the F-actin network layer is higher than without a bead. Scale bars: $5 \mu \mathrm{m}$ (horizontal and vertical). Modified and reprinted with permission from Nöding, Schön et al. Copyright 2018 American Chemical Society. ${ }^{[160]}$ 
First, the obtained micrographs show that the actin network organizes around the bead to incorporate the bead into the F-actin network. Second, it also shows that the tracer particles are fully enclosed by F-actin and not caged between the lipid bilayer and the F-actin network. Therefore, the bead experiences the movement and viscoelastic response of the encaging actin network. Third, the $z$-stacked images also reveal that the height of the F-actin layer around the beads is locally higher than attached F-actin networks without beads (cf. chapter 4.2.2). It seems that the attached layer which constitutes the bottom site of the cage contributes its raised stiffness to the other sides, thus leading to the change of viscoelastic properties as this is the main difference to $3 \mathrm{D}$ actin gels.

For microrheological experiments, VPT was performed as described in chapter 3.6.4. The recorded viscoelastic frequency spectra are shown in Figure 4.29.
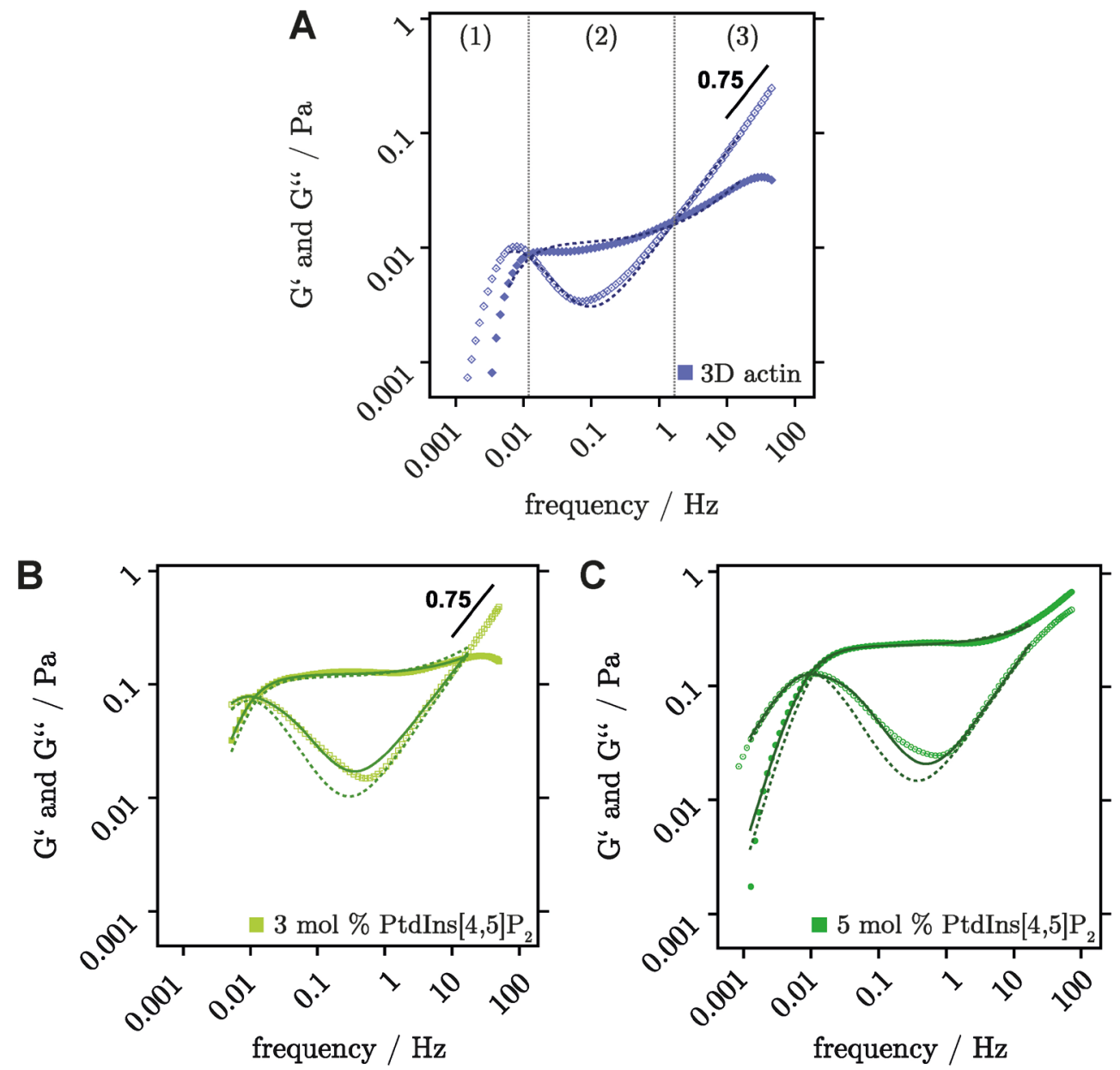

Figure 4.29: Frequency dependent viscoelastic properties of MACs in comparison to 3D F-actin gels. ${ }^{[160]}$ In all cases the storage modulus $G^{\prime}$ is displayed as filled symbols and $G$ " as open symbols. Lines display a fit according to the theoretical model from Plagge et al. The dashed lines display a fit including only one kind of cross-link and the solid line concerning two cross-links. ${ }^{173]}$ A: F-actin networks in 3D gels $(8-23 \mu \mathrm{M})$ show a plateau modulus of $G_{0}=9 \mathrm{mPa}(N=5)$. The power law scaling behavior with an exponent of 0.75 is illustrated 
as a black line. The frequency spectra are divided into three regimes: the low frequency regime (1), the plateau regime (2) and the high frequency regime (3). B: A plateau modulus of $G_{0}=0.135 \mathrm{~Pa}(N=6)$ is determined for F-actin networks attached to a lipid bilayer doped with $3 \mathrm{~mol} \%$ PtdIns[4,5] $\mathrm{P}_{2}$ via ezrin T567D. C: Ezrin mediated MACs on a lipid bilayer with $5 \mathrm{~mol} \% \mathrm{PtdIns}[4,5] \mathrm{P}_{2}$ display a higher plateau modulus of $G_{0}=0.233 \mathrm{~Pa}(N=9) . N$ displays the averaged bead trajectories. Modified and reprinted with permission from Nöding, Schön et al. Copyright 2018 American Chemical Society. ${ }^{[160]}$

In Figure 4.29 A, a typical spectrum of frequency dependent viscoelastic properties is shown in form of the storage modulus $G^{\prime}$ (filled symbols) and the loss modulus $G^{\prime \prime}$ (open symbols). As already mentioned in chapter 3.6.4, three main regimes are obtained. In the low frequency regime (1) dynamic processes like binding kinetics or diffusion processes dominate. The plateau regime (2) differs from both other regimes as the elastic response prevails the viscous part $\left(G>G^{\prime}\right)$. The last regime found at high frequencies (3) is mostly dominated by mechanical properties of single filaments. It typically shows a power law scaling exponentially scaled with $3 / 4$ as illustrated in Figure 4.29 A/B. Even in an almost 2D system of an attached MAC these three regimes are clearly distinguishable. The plateau modulus $G_{0}$ is a characteristic property as it refers to the stiffness of the measured system. This value can be extracted by determining the magnitude of the storage modulus at the minimum of the loss modulus in this region. For 3D F-actin gels (Figure $4.29 \mathrm{~A}$ ) a plateau modulus of $G_{0}=9 \mathrm{mPa}$ was found (cf. Figure 4.30). Compared with literature the measured value of this work is three times lower. ${ }^{[210]}$ These values depend on the preparation of the networks and in the work of Gardel et al. the use of phalloidin was waived which could lead to this discrepancy.

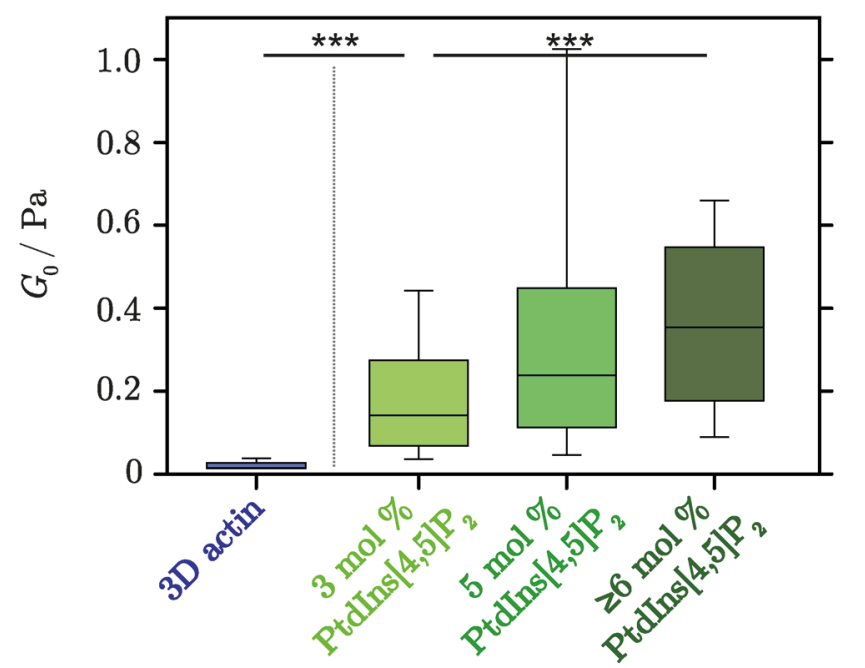

Figure 4.30: Change of the plateau modulus $G_{0}$ of actin model-systems as function of membrane-attachment and further on pinning point density. ${ }^{[160]}$ While 3D F-actin network gels $(8-23 \mu \mathrm{M})$ show a system stiffness of $G_{0}=9 \mathrm{mPa}(N=8)$, the attachment to a lipid bilayer doped with $3 \mathrm{~mol} \% \operatorname{PtdIns}[4,5] \mathrm{P}_{2}$ lead to a 15 -fold increase of the plateau modulus to $G_{0}=0.135 \mathrm{~Pa}(N=306)$. Higher pinning point densities lead to an even more pronounced 
increase: $\quad 5 \mathrm{~mol} \% \operatorname{PtdIns}[4,5] \mathrm{P}_{2}: \quad G_{0}=0.233 \mathrm{~Pa} \quad(N=14) . \quad \geq 6 \mathrm{~mol} \% \quad \operatorname{PtdIns}[4,5] \mathrm{P}_{2}$ : $G_{0}=0.348 \mathrm{~Pa}(N=243)$. Boxes represent data from $25^{\text {th }}$ to $75^{\text {th }}$ percentile, solid line displays the median and whiskers extent to the $10^{\text {th }}$ and $90^{\text {th }}$ percentile. A two sample Mann-Whitney U-test was performed. The null hypothesis was rejected in both cases at the $0.1 \%$ significance level (***). Modified and reprinted with permission from Nöding, Schön et al. Copyright 2018 American Chemical Society. ${ }^{[160]}$

The subsequent attachment of the F-actin network via ezrin T567D to a lipid bilayer containing $3 \mathrm{~mol} \% \operatorname{PtdIns}[4,5] \mathrm{P}_{2}$ resulting in the formation of an MAC leads to a 15-fold increase of the plateau modulus up to $G_{0}=0.135 \mathrm{~Pa}$ as shown in Figure 4.30. It is unlikely that this great increase in the overall stiffness $G_{0}$ is only a function of a barely more condensed F-actin network as the plateau modulus of densely cross-linked networks only has a dependence of $G_{0} \propto c_{a}{ }^{2.2}$ concerning that a higher G-actin concentration $\left(c_{\mathrm{a}}\right)$ also leads to more condensed F-actin networks. Therefore, it is suggested that also the binding to the $C$-ERMAD of ezrin, which confines the movement of the actin filaments, increases the stiffness of the MAC. An even higher stiffness of $G_{0}=0.223 \mathrm{~Pa}$ was found when offering more pinning points due to the use of $5 \mathrm{~mol} \% \operatorname{PtdIns}[4,5] \mathrm{P}_{2}$. This supports the suggestion that the confinement of movement by ezrin binding increases the stiffness of the MAC. When offering a complete surface coverage of ezrin T567D cross-links $\left(\geq 6 \mathrm{~mol} \% \operatorname{PtdIns}[4,5] \mathrm{P}_{2}\right.$, cf. Figure 4.5, chapter 4.1.3) the plateau modulus increases finally up to $G_{0}=0.348 \mathrm{~Pa}$.

The ability of cross-linkers to strengthen networks is usually described in literature as a power law scaling of the plateau modulus $G_{0} \propto R^{\mathrm{x}}$. $R$ displays the ratio of cross-linkers $\left(c_{\mathrm{c}-1}\right)$ to $\mathrm{G}$-actin $\left(c_{\mathrm{a}}\right)$ with $\mathrm{R}=c_{\mathrm{c}-\mathrm{l}} / c_{\mathrm{a}}$. As the number of ezrin cross-links is determined by the pinning point density of PtdIns[4,5] $\mathrm{P}_{2}$ it is assumed that the content of $\operatorname{PtdIns}[4,5] \mathrm{P}_{2}$ is equivalent to $R$. A relation of $G_{0} \propto\left(\chi_{\mathrm{PtdIns}[4,5] \mathrm{P}_{2}}\right)^{1.5}$ was determined which is lower than the found relation in literature for $3 \mathrm{D}$ F-actin networks. For these $3 \mathrm{D}$ networks a power law coefficient of 2 was found, ${ }^{[211]}$ while for an F-actin network attached to a lipid monolayer via biotin-streptavidin binding a coefficient of 1 was reported. ${ }^{[74]}$ For completely affinely deforming networks in 2D, a simulation study also showed a power law scaling of the plateau modulus on the cross-linker density of $G_{0} \propto \chi^{\left.1.0 .{ }^{2} 212\right]}$ As shown in chapter 4.2.3, the node density $\nu$ of MACs ascends nearly linearly with increasing pinning point density inside the lipid bilayer. Interconnected with the dependency of the plateau modulus $G_{0}$ on the pinning point density a scaling behavior of $G_{0}$ with the node density, the resulting $2 \mathrm{D}$ projection as characterized in chapter 4.2.3, of $G_{0} \propto \nu^{1.5}$ is found.

As already mentioned, in the high frequency regime (Figure $4.29 \mathrm{~A}(3)$ ) the viscoelastic response is prevailed by bending mode relaxations which derive from single filament mechanics and typically result in a power law exponent of 3/4. ${ }^{[171,213]}$ In our experiments 
an exponent of 0.60 to 0.86 was found both in the viscoelastic properties of $3 \mathrm{D}$ network gels and MACs. The slight difference in scaling of the real part at higher frequencies could be a result of inertia effects. ${ }^{[214]}$

In the low frequency regime (Figure $4.29 \mathrm{~A}(1)$ ) the elastically stored energy is dissipated both for 3D actin gels as well as for MACs. Slow dynamics like bindingunbinding processes or filament reptation are a possible origin for this energy dissipation. ${ }^{[171,215]}$ Filament reptation, thus diffusion along the contour length, is highly dependent on the filament length. To test this, the F-actin network was modified by a decreasing filament length. The protein gelsolin was used to intentionally shorten the mean filament length as gelsolin is a well-known F-actin capping protein. ${ }^{[216-218]}$
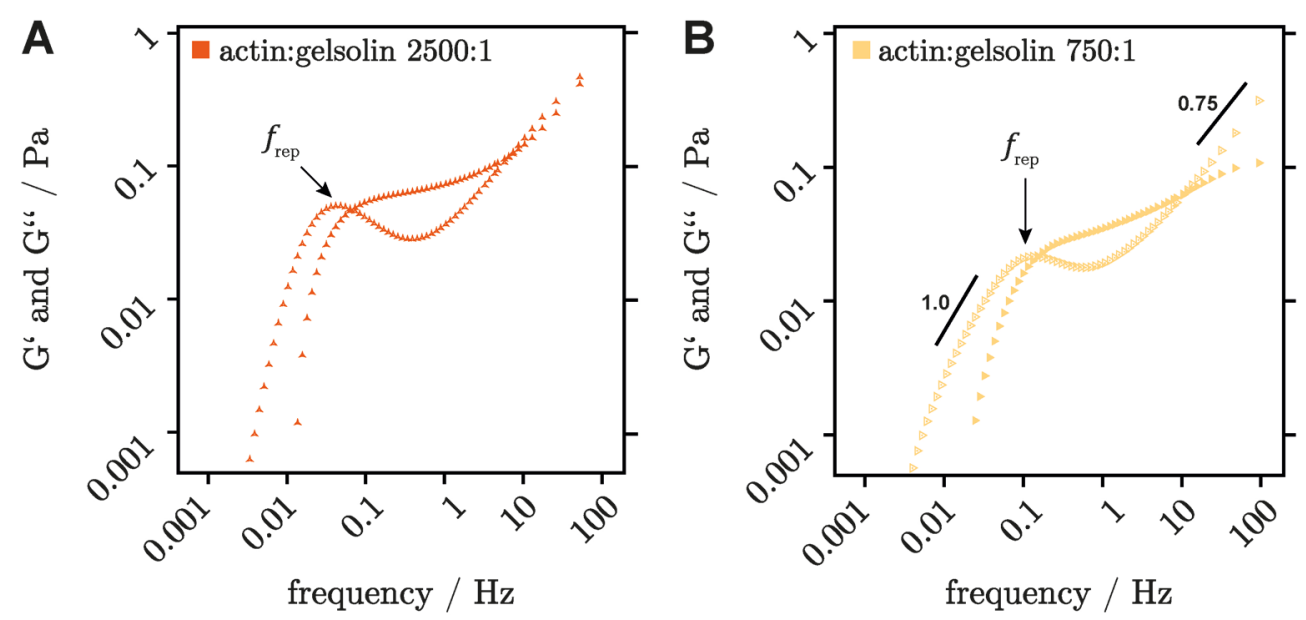

Figure 4.31: Frequency dependent viscoelastic properties of 3D F-actin networks with shortened mean filament length by gelsolin. ${ }^{[160]}$ The storage modulus $G^{\prime}$ is displayed as filled symbols and $\boldsymbol{G}^{\prime \prime}$ as open symbols. A: Viscoelastic properties of $23 \mu \mathrm{M}$ entangled F-actin networks polymerized together with gelsolin in a ratio of 2500:1 (actin:gelsolin, orange, $N=5$ ) show a reptation frequency of $f_{\text {rep }}=0.040 \mathrm{~s}^{-1}$. B: Increasing the gelsolin ratio up to 750:1 (actin:gelsolin, $c_{\mathrm{a}}=26 \mu \mathrm{M}$ ), thus shortening the mean filament length even more, shifts the reptation frequency up to $f_{\text {rep }}=0.128 \mathrm{~s}^{-1}(N=5)$. Typical scaling behavior of 1.0 in the low and 0.75 in the high frequency regime is pointed out in $(\mathbf{B}) . N$ is the number of averaged bead trajectories. Modified and reprinted with permission from Nöding, Schön et al. Copyright 2018 American Chemical Society. ${ }^{[160]}$

A clear shift of the reptation frequency of 3D F-actin gels as presented in Figure 4.29 from $f_{\text {rep }}=0.008 \mathrm{~s}^{-1}$ via $f_{\text {rep }}=0.040 \mathrm{~s}^{-1}$ (actin:gelsolin 2500:1, Figure $4.31 \mathrm{~A}$ ) up to $f_{\text {rep }}=0.128 \mathrm{~s}^{-1}$ (actin:gelsolin 750:1, Figure $4.31 \mathrm{~B}$ ) is obtained by adding and increasing the gelsolin ratio. According to Morse, ${ }^{[171]}$ the observed increase of the crossover frequency $f_{\text {rep }}$ in the experiments displayed in Figure 4.31 can be expected with shorter filaments. The relationship between the average filament contour length $(L)$ and the reptation frequency or rather the reptation time is predicted by his theory: ${ }^{[171]}$

$$
f_{\text {rep }}=\tau_{\text {rep }}{ }^{-1}
$$




$$
\tau_{\text {rep }}=\frac{L^{3} \cdot 2 \eta}{\pi \cdot k_{\mathrm{B}} T \cdot \ln \left(\frac{\xi}{d}\right)}
$$

With the assumption that the solvent viscosity $\eta=0.891 \mathrm{mPa} \cdot \mathrm{s}$, the mesh size $\xi=900 \mathrm{~nm}$ and the F-actin diameter of $d=8 \mathrm{~nm}$ do not differ exceptionally within the experiments of untreated 3D F-actin gels and gelsolin treated F-actin the mean length of the filaments $(L)$ can be determined. For untreated F-actin networks an average filament length of $L=16.3 \mu \mathrm{m}$ was obtained. According to literature, this corresponds quite well to typically F-actin filament lengths in vitro of about 10-20 $\mathrm{mm} .{ }^{[196,197]}$ Furthermore, this is in good accordance with the observed filament length of F-actin in 3D gels which were determined to be $(17 \pm 3) \mu \mathrm{m}$ in chapter 4.2.1. As already pointed out in chapter 4.2.3, this value is higher compared to actin filament lengths found in the cortex of living cells which show an average length below $2 \mu \mathrm{m} .{ }^{[205]}$ In case of gelsolin treated F-actin networks shorter filament lengths of $L=9.5 \mu \mathrm{m}$ (actin:gelsolin 2500:1) and $L=6.5 \mu \mathrm{m}$ (actin:gelsolin 750:1) were found. As already expected, the gelsolin treatment significantly shortens the filaments and in turn this leads to a shift of the low frequency crossover to higher frequencies with $f_{\text {rep }} \propto L^{-3}$ according to equation 4.6. This finding verifies the presence of filament reptation dynamics in the low frequency regime of 3D F-actin gels. In case of membrane-attached F-actin networks, in the rheological data of the low frequency regime, bindingunbinding kinetics of the ezrin-F-actin attachment, additionally to the filament reptation, can be found. To get access to these distinct unbinding rates, the spectral properties of these viscoelastic cortices were fitted by a recently developed theory which describes reversibly cross-linked networks of semi-flexible polymers within a non-affine concept by Plagge et al.[173] Besides a basic assumption that actin filaments are inextensible, this model is based on the theory that linear bending deformations of an actin filament occur inside an effective "tube-like" medium formed by the surrounding network. This is justified as bending modes are by far softer than stretching of filaments. The surrounding medium is described as harmonic potential acting on the filament while it is assumed that network straining is inducing a local position change of the filament and is not changing the actual length. The final coupling of the surrounding medium to the actin filament is assumed to happen through reversible cross-links, where the thermal energy is comparable to the strength of the respective cross-link. In case of our experiments the cross-links are equated to entanglements of F-actin filaments (in 3D F-actin gels). In case of attached MACs, the cross-links are equated to a sum of the still present entanglements and the ezrin binding to filamentous actin. As the lifetime of ezrin bonds is found to be in the range of a second, it is expected to observe distinct relaxation times in the rheological spectra. ${ }^{[219]}$ It is assumed that the cross-links exist in a rough periodic 1D energy landscape distorted by external force rendering escape from the potential-well preferential in one direction from force 
dependent rates. This implies that the rough energy landscape generates friction that can be represented by a dashpot. Thus, the response function, representing the viscoelasticity of the effective medium which is the F-actin network, consists of Maxwell elements. The number of Maxwell elements is equal to the number of cross-link types with different binding-unbinding rates. Developing the model, for one type of cross-link a simple equation 4.7 can be formulated for the effective modulus $G^{*}$.

$$
\frac{1}{G^{*}}=\frac{1}{G_{\mathrm{S}}}+\frac{1}{i \eta \omega}
$$

$G_{\mathrm{s}}$ is the modulus with permanent cross-links and $\eta$ is the friction coefficient relating to binding-unbinding of cross-linkers, which is simulated by a sliding along the filaments. The permanent cross-link modulus $G_{\mathrm{S}}$ can be written as sum over filament bending modes with wavenumber $q_{\mathrm{n}}$ as

$$
\frac{1}{G_{\mathrm{S}}}=\frac{1}{G_{0}} \sum_{n} \frac{\sin ^{2}\left(q_{n} L / 2\right)}{\left(L q_{n}\right)^{4}+i \omega / \omega_{0}}
$$

As before, $G_{0}$ is the plateau modulus and $L$ the filament contour length while $\omega_{0}$ is the cross-over frequency to the high frequency branch. This leads at high frequencies to the typical $G_{\mathrm{S}} \approx(i \omega)^{3 / 4}$ scaling describing single filament mechanics and at low frequency a plateau at $G_{0}$. Concerning the limiting cases for the real (equation 4.9) and the imaginary part (equation 4.10) a simplified formula (equation 4.11 and 4.12 ) can be formulated:

$$
\begin{gathered}
G^{\prime}{ }_{\mathrm{S}} \rightarrow G_{0}\left\{\begin{aligned}
0.38 \cdot \alpha_{0} \cdot\left(\frac{\omega}{\omega_{0}}\right)^{\frac{3}{4}}, & \omega \gg \omega_{0} \\
1, & \omega \ll \omega_{0}
\end{aligned}\right. \\
G^{\prime \prime}{ }_{\mathrm{S}} \rightarrow G_{0}\left\{\begin{aligned}
0.92 \cdot \alpha_{0} \cdot\left(\frac{\omega}{\omega_{0}}\right)^{\frac{3}{4}}, & \omega \gg \omega_{0} \\
\left(\frac{\omega}{\omega_{0}}\right), & \omega \ll \omega_{0}
\end{aligned}\right.
\end{gathered}
$$

The simplified formulas show numerical factors which derive from $i^{3 / 4}$ and introduce a scaling parameter $\alpha_{0}$.

$$
\begin{gathered}
G^{\prime}{ }_{\mathrm{S}}=G_{0} \cdot\left(1+0.38 \cdot \alpha_{0} \cdot\left(\frac{\omega}{\omega_{0}}\right)^{\frac{3}{4}}\right) \\
G^{\prime \prime}{ }_{\mathrm{S}}=G_{0} \cdot\left(\left(0.92 \cdot \alpha_{0}\right)^{-1} \cdot\left(\frac{\omega}{\omega_{0}}\right)^{-\frac{3}{4}} \cdot\left(\frac{\omega}{\omega_{0}}\right)^{-1}\right)^{-1}
\end{gathered}
$$

Splitting for real and imaginary parts, one writes

$$
G^{\prime}=(\eta \omega)^{2} \cdot \frac{G^{\prime}}{D}
$$




$$
G^{\prime \prime}=\alpha_{1} \eta \omega \cdot \frac{\left(\left(G_{S}^{\prime}\right)^{2}+G^{\prime \prime}{ }_{S}\left(G^{\prime \prime}{ }_{S}+\eta \omega\right)\right)}{D}
$$

with

$$
D=\left(G^{\prime}\right)^{2}+\left(G^{\prime \prime}{ }_{S}+\eta \omega\right)^{2} .
$$

The newly introduced parameter $\alpha_{1}$ allows shifting the imaginary part relatively to the real part which leads to five fit parameters in total: $G_{0}, \omega_{0}, \eta, \alpha_{0}$ and $\alpha_{1}$. The resulting fits to the data using the introduced model are displayed in Figure 4.29 as dashed lines. The unbinding timescale is given by $\tau=\eta / G_{0}$. For ezrin mediated membrane-attached F-actin networks the wing right beside the peak of $G^{\prime \prime}$ in the low frequency regime is broader than it is expected from only one Maxwell element. But in our experiments with attached MACs we expect two types of cross-links: First, the entanglement within the F-actin network as described and second, the binding-unbinding rate of the network to the lipid bilayer which is separated into binding-unbinding rates of ezrin-actin as well as ezrin-PtdIns[4,5] $\mathrm{P}_{2}$. Therefore, additional timescales which relate to this ezrin linkage of the F-actin network to the lipid bilayers are expected and are considered by adding a second Maxwell element (equation 4.16 and 4.17).

$$
G^{\prime}=G_{1}^{\prime}+\alpha_{3} \cdot G_{2}^{\prime}
$$

and

$$
G^{\prime \prime}=G^{\prime \prime}{ }_{1}+\alpha_{2} \cdot G^{\prime \prime}{ }_{2}
$$

As the real part did not show a clear second plateau in presence of two Maxwell elements two additional scaling factors $\alpha_{3}$ and $\alpha_{2}$ were introduced. We found $\alpha_{3} \ll \alpha_{2}$. In total, the model consists of the fit parameters $G_{0}, \omega_{0}, \eta_{1}$ and $\eta_{2}$ and the scaling parameters $\alpha_{0}, \alpha_{1}, \alpha_{2}$ and $\alpha_{3}$. The used set of parameters is provided in the appendix (chapter 7.3) and the resulting fit to the data is displayed as solid line in Figure 4.29 $\mathrm{B} / \mathrm{C}$. Utilizing this fit, the obtained reptation frequency was used to determine the

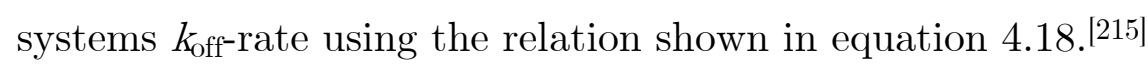

$$
f_{\text {rep }}=\frac{1}{\tau}=\frac{G_{0}}{\eta} \approx \frac{k_{\text {off }}}{2 \pi}
$$

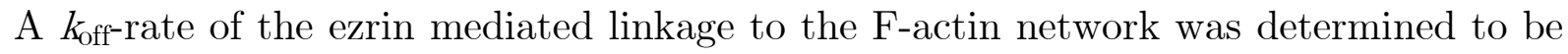
around $0.03 \mathrm{~s}^{-1}$. Fritzsche et al. reported a higher value for the unbinding rate by using single-molecule studies as they studied the ezrin binding in vivo. ${ }^{[219]}$ Other in vitro studies determined $k_{\mathrm{off}}=1.3 \mathrm{~s}^{-1}$ by means of force spectroscopy. [55] In total, the

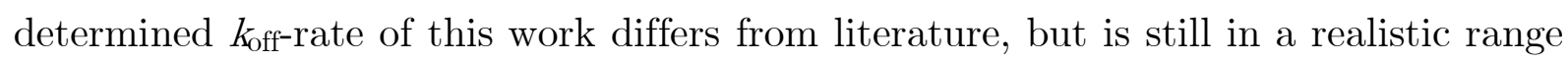
as it must be considered that the totally crowded situation on the artificial lipid bilayer could lead to a rebinding effect which would effectively strengthen the F-actin bonding. 


\section{Temperature Dependency of Detected Unbinding Rate Constants}

Utilizing a temperature-controlled measuring chamber which controls the temperature in two different ways (at the objective and the chamber bottom), it was possible to quantify the viscoelastic properties of the model system using the $\operatorname{Ptd} \operatorname{Ins}[4,5] \mathrm{P}_{2}$-ezrin T567D-actin binding complex (lipid bilayer was doped with $3 \mathrm{~mol} \% \operatorname{Ptd} \operatorname{Ins}[4,5] \mathrm{P}_{2}$ ) at three steady temperatures starting with $12{ }^{\circ} \mathrm{C}$ via $27{ }^{\circ} \mathrm{C}$ up to $36{ }^{\circ} \mathrm{C} .36{ }^{\circ} \mathrm{C}$ as the upper limit was chosen to secure an intact binding complex as well as it represents physiological conditions. The resulting unbinding rate constants, determined as shown before, were plotted logarithmically against the reciprocal of the temperature to achieve a typical Arrhenius plot (Figure 4.32). From fitting the Arrhenius equation (equation 4.19) the transition barrier height $\Delta E_{\mathrm{a}}$ as well as the preexponential factor $A$ could be estimated.

$$
\ln k_{\mathrm{off}}=\ln A-\frac{\Delta E_{a}}{R \cdot T}
$$

Using the ideal gas constant $R$, a transition barrier height of $\Delta E_{\mathrm{a}}=(31 \pm 12) \mathrm{kJ} \cdot \mathrm{mol}^{-1}$ and a relating preexponential factor $A=(1660 \pm 8299) \mathrm{s}^{-1}$ was found. The rather low barrier height underlines the transient nature of the binding system. The detected value for the preexponential factor is more roughly, as for a more precise determination additional temperature points more far away from the detected region should have been measured which is, however, experimentally challenging. Even the highest value within the error margin appear to be very small, because simple unimolecular reactions concerning small molecules show a frequency factor of about $10^{13} \mathrm{~s}^{-1}$, deriving from collision theory.

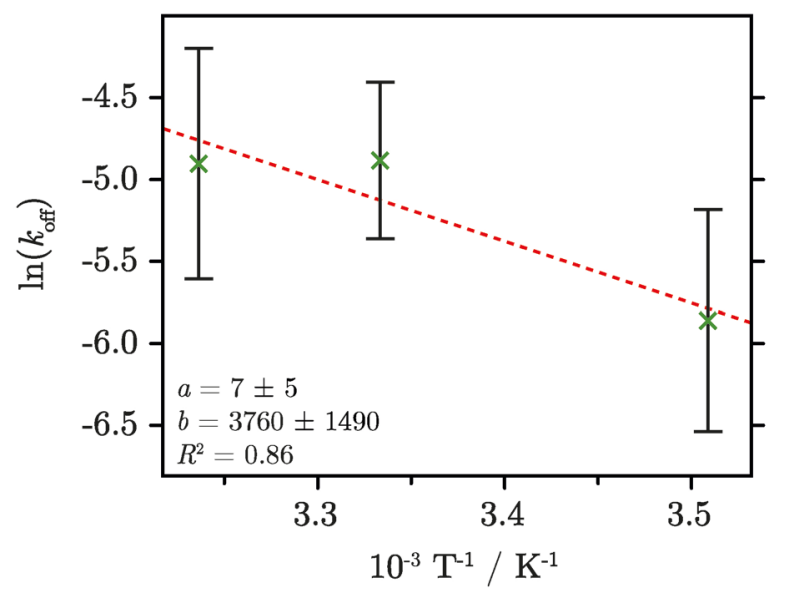

Figure 4.32: Arrhenius plot showing the temperature dependency of the PtdIns $[4,5] P_{2}$-ezrin-actin binding complex. In an experiment with a lipid bilayer containing $3 \mathrm{~mol} \% \mathrm{PtdIns}[4,5] \mathrm{P}_{2}$ the viscoelastic properties were determined by means of passive microrheology at three different temperatures: $12{ }^{\circ} \mathrm{C}, 27{ }^{\circ} \mathrm{C}$ and $36{ }^{\circ} \mathrm{C}$. Data points display the mean value $(N=5)$ and the respective standard deviation. The linear Arrhenius fit (red) with displayed fitting parameters revealed an activation energy of $\Delta E_{\mathrm{a}}=(31.3 \pm 12.4)$ 
$\mathrm{kJ} \cdot \mathrm{mol}^{-1}$ and a preexponential factor $A=(1660 \pm 8299) \mathrm{s}^{-1}$. Graphic was kindly provided by Helen Nöding. ${ }^{[166]}$

Nevertheless, the determined transition barrier height appears to be in an expected regime for transient cross-links which can be related to literature. The transition barrier height of the dissociation of F-actin and heavy meromyosin, another cross-link which is the actin binding subunit of a myosin motor protein without the light protein chains, was found to be $\Delta E_{\mathrm{a}}=35 \mathrm{~kJ} \cdot \mathrm{mol}^{-1}=12.6 \mathrm{k} \mathrm{B} T$ which is close to the detected value of this work. ${ }^{[220]}$ Conversely, Lieleg et al. could not find any transition barrier height for the same system (actin-heavy meromyosin).[221] Also, for the binding system of $\alpha$-actinin-actin a transition barrier height of $\Delta E_{\mathrm{a}}=(119.2 \pm 4.4) \mathrm{kJ} \cdot \mathrm{mol}^{-1}$ was found in microrheological experiments which is more than three times higher than the detected value for ezrin-actin binding system.[222]

The obtained results also support that the local maximum in the low frequency regime arise from transient binding kinetics which can be related to the binding-unbinding of PtdIns[4,5] $\mathrm{P}_{2}$-ezrin-actin complex. Generally, the detected value for the transition barrier height must be handled with care as there are some factors which remain unclear. By changing the temperature, the system is exposed to changes which were not considered, like temperature induced fluctuations of the filaments, membrane properties like fluidity or changed solvent viscosity. However, the transient nature of the PtdIns $[4,5] \mathrm{P}_{2}$-ezrin-actin binding complex is verified by these experiments. It was also shown that the binding complex is temperature dependent and that dissociation still occurs at low temperatures of $12{ }^{\circ} \mathrm{C}$.

\subsubsection{Viscoelastic Properties of non-physiologically attached F-actin networks}

- Microrheology experiments were performed in cooperation with Helen Nöding as part of project $A 17$ of the collaborative research center $937^{[166]}$ -

The aim of using a second binding complex was to determine the viscoelastic properties by means of passive microrheology and characterize the possible change induced by a different binding complex, which is used to attach the F-actin network to the lipid bilayer. The biotin-avidin bond is known to be the strongest biochemical non-covalent bond $\left(K_{\mathrm{D}} \approx 10^{-15} \mathrm{M}\right)$. ${ }^{[223]}$ Therefore, a comparison of the viscoelastic properties of F-actin networks attached via the physiological binding system (cf. chapter 4.3.1) and via the biotin-avidin linkage was performed. In contrast to experiments shown in chapter 4.3.1, F-actin networks were attached in a non-physiological way via a 
biotin-neutravidin binding complex to a lipid bilayer. Neutravidin is a protein based on avidin, similar to streptavidin, and has four accessible biotin-binding sites.[224] Neutravidin and streptavidin provide a near-neutral isoelectric point in contrast to avidin and neutravidin shows lower non-specific binding than streptavidin and avidin. ${ }^{[225]}$ The binding complex is built using biotinylated lipids (cap-bio-DOPE, cf. 3.1.1.2) in the first step to bind neutravidin to the lipid bilayer. Furthermore, biotinylated G-actin in a ratio of 1:5 (bio-G-actin:G-actin) was polymerized to achieve biotinylated F-actin networks. These networks were then attached to the neutravidin incubated lipid bilayer containing cap-bio-DOPE. Using incorporated tracer particles, again polystyrene beads of a $1 \mu \mathrm{m}$ radius, the frequency dependent viscoelastic properties were determined by means of video particle tracking.
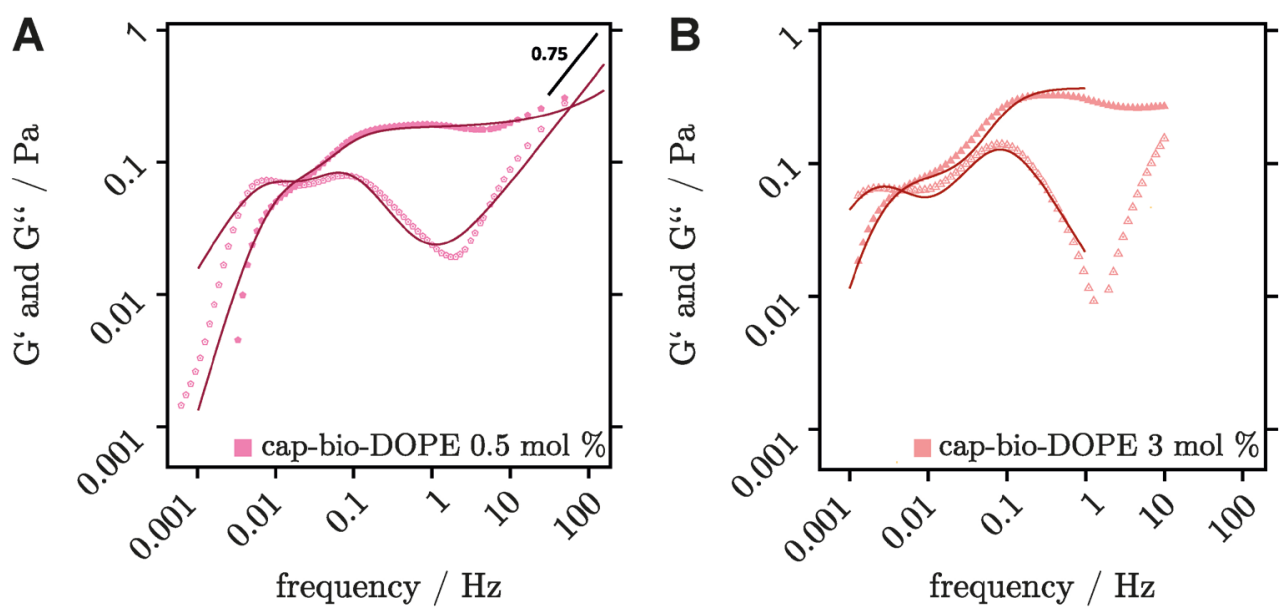

Figure 4.33: Frequency dependent viscoelastic properties of biotin-neutravidin mediated, membrane-attached MACs. A: MACs attached via neutravidin to a lipid bilayer containing $0.5 \mathrm{~mol} \%$ cap-bio-DOPE reveal a plateau modulus of $G_{0}=0.083 \mathrm{~Pa}$. B: Providing more pinning points by increasing the cap-bio-DOPE ratio to $3 \mathrm{~mol} \%$ led to an increased plateau modulus of $G_{0}=0.29 \mathrm{~Pa}$. The solid line in $(\mathbf{A})$ display the fit which was produced using the parameters shown in chapter 7.3. In case of $3 \mathrm{~mol} \%$ the solid line displays a fit, which was modeled by a fix parameter set (also chapter 7.3) for the low frequency regime as no converged fit for the whole frequency spectrum could be found. Interestingly, both graphs show two peaks in the low frequency regime of the loss modulus.

Figure 4.33 reveals significant differences in the viscoelastic properties while the absolute stiffness is found to be in the same regime than the physiological binding system. While the attachment via the physiological binding complex showed a 14-fold increase of the plateau modulus, the biotin-neutravidin binding increased the plateau modulus 9 -fold up to $G_{0}=0.083 \mathrm{~Pa}(N=1,0.5 \mathrm{~mol} \%$ cap-bio-DOPE) compared to $3 \mathrm{D}$ actin gels. A dependence of $G_{0}$ on the pinning point density as it was found using the physiological binding system was also observed using this second membrane attachment via neutravidin. When using $3 \mathrm{~mol} \%$ bio-cap-DOPE the plateau modulus was increased up to $G_{0}=0.29 \mathrm{~Pa}(N=4)$. A possible explanation for the larger 
increase in contrast to the physiological binding system could be an even more restricted movement of the filaments as the binding complex is almost as strong as a covalent bond and the neutravidin-avidin complex therefore provides a stronger cross-link. Comparing with the results of the previous section, the results of both binding-complexes support the assumption that the attachment is not solely responsible for the increase of the plateau modulus. It is hypothesized that the confinement of F-actin movement is also responsible for the increase of $G_{0}$ which is supported by a further increase in stiffness by further restricting the F-actin movement. Therefore, the pinning point density as well as the nature of the cross-link have a great impact on the viscoelastic properties of the F-actin network.

Concerning the pinning point density related scaling, we found $G_{0} \propto\left(\chi_{\text {cap-bio-Dope }}\right)^{0.3}$ in contrast to $G_{0} \propto\left(\chi_{\operatorname{PtdIns}[4,5] \mathrm{P}_{2}}\right)^{1.5}$. This value differs from comparable studies found in literature. These studies both found a linear scaling of $G_{0} \propto\left(\chi_{\text {streptavidin }}\right)^{1.0}$ using a biotin-streptavidin binding complex. ${ }^{[74,226]}$ However, it must be noted that the stiffness of the biotin-neutravidin binding complex is at the limit of the measuring technique for these experiments ${ }^{[166]}$ which implies that a systematically lower plateau modulus was measured and therefore the scaling factor is underestimated.

In the low frequency regime, the most significant change in contrast to the physiological binding system is observed. While the data of the physiological binding complex shows one broadened peak with a soft shoulder to lower frequencies, the data of the biotin-neutravidin binding complex exhibits two distinct peaks. For both peaks two independent unbinding rate constants were determined: $k_{\text {off }}^{1}=0.076 \mathrm{~s}^{-1}$ and $k_{\text {off }}^{2}=0.009 \mathrm{~s}^{-1}$. As we were not able to distinguish the processes experimentally, it is assumed that $k_{\text {off }}^{1}$ derives from un-entanglement of F-actin filaments and $k_{\text {off }}^{2}$ from the unbinding of the biotin-neutravidin-biotin complex. The reason for this assignment is that the relaxation of the entanglement in 2D (neutravidin mediated, 3 mol \%) is way higher than in entangled 3D networks $\left(k_{\text {off }}^{3 \mathrm{D}}=0.05 \mathrm{~s}^{-1}\right)$ as there are less entanglement points in $z$-direction and therefore, the system can relax in shorter time. Furthermore, the way shorter filament lengths in 2D (cf. chapter 4.2) may lead to a higher $k_{\text {off }}^{1}$ which is supported by the observed results using the capping protein gelsolin shown before. Therefore, the second unbinding rate is attributed to the unbinding of the neutravidin-complex. While for streptavidin and avidin an unbinding constant of $k_{\text {off }} \approx 5 \cdot 10^{-6} \mathrm{~s}^{-1}$ was presented in literature, for neutravidin a lower value of $k_{\text {off }} \approx 5 \cdot 10^{-}$ ${ }^{4} \mathrm{~S}^{-1}$ was reported. ${ }^{[227-230]}$ Regarding the theoretically simplest case that neutravidin is bound by 2 biotin molecules estimates the rate constant to $k_{\mathrm{off}}=2 \cdot 5 \cdot 10^{-4} \mathrm{~s}^{-1} \approx 1 \cdot 10^{-3} \mathrm{~s}^{-}$ 1. Compared to the observed values in this work this value is 9-times lower than the $k_{\text {off }}^{2}$ value resulting from $0.5 \mathrm{~mol} \%$ cap-bio-DOPE data $\left(k_{\text {off }}^{2}(0.5 \mathrm{~mol} \%)=0.09 \mathrm{~s}^{-1}\right)$ and 2 -times lower than the value resulting from $3 \mathrm{~mol} \%$ data $\left(k_{\mathrm{off}}^{2}(3 \mathrm{~mol} \%)=0.002\right.$ 
$\mathrm{s}^{-1}$ ). In contrast to these findings, further experiments using molecular force spectroscopy showed way higher unbinding rate constants of $k_{\text {off }} \approx 0.75 \mathrm{~s}^{-1}$ for biotin/avidin binding system or $k_{\text {off }} \approx 10^{-2}$ (high loading rate) and $k_{\text {off }} \approx 1.7 \cdot 10^{-5}-\mathrm{s}^{-1}$ (low loading rate) for the biotin/streptavidin binding system. ${ }^{84,231,232]}$ The difference between measured unbinding rates in our work and the rates reported in literature implies that the assumed simple binding model might not be sufficient to describe complex binding situations as found in this model system. Neutravidin has four binding

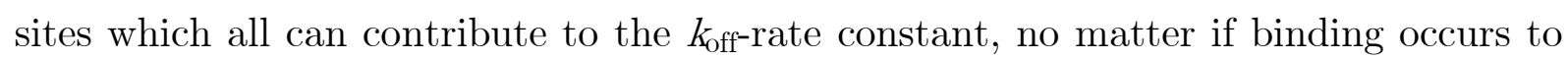
biotinylated actin or a biotinylated lipid. In the previously described comparison only the simplest case, one binding site attached actin, one to the lipid, is considered to establish a connection between lipid bilayer and F-actin network. In the observed viscoelastic properties, an interplay of two, three or four binding sites per neutravidin occurs leading to the fact that the described comparison is probably an underestimation of the unbinding rate constant. This assumption fits not only for the biotin-neutravidin binding complex, but also for the physiological binding via PtdIns $[4,5] \mathrm{P}_{2} /$ ezrin.

Concluding, the comparison with the experiments of a non-physiological binding complex support the finding of the transient nature of the physiological binding complex as significant differences between both viscoelastic properties could be observed. Furthermore, the assumption of overlying binding dynamics within the broadened peak in the low frequency regime is supported, as in the experiments with biotin-neutravidin binding system both kinetics are sufficiently different to reveal two distinct peaks in this regime of the viscoelastic properties. 


\subsubsection{Force-Indentation Experiments on F-actin Networks}

In order to characterize the influence of F-actin attachment on the underlying lipid bilayer, force indentation experiments on membranes with and without attached F-actin network will be conducted. For this, porous substrates were used on which giant unilamellar vesicles (GUVs) were spread to form pore spanning lipid bilayers (PSLBs).[233-235] The free-standing parts of the membrane (pore spanning membrane, PSM) were then indented by a cantilever tip to determine the elastic response of the lipid bilayer by force indentation experiments (chapter 3.5.1.1). The resulting slope $\left(k_{\text {app }}\right)$ of force-indentation curves were used to determine the lateral tension of the PSLBs. Subsequently, a minimal actin cortex was attached to the lipid bilayer and the lateral tension of PSLBs was determined again in order to track a possible impact of F-actin attachment.

\subsubsection{Electrostatically Bound F-actin Networks}

- Indentation experiments have been carried out by Peter Mühlenbrock as part of his master thesis -

The first approach in determining the F-actin induced change of the lipid bilayer's mechanical properties was pursued with a non-physiological binding system. As actin filaments have a negative net charge, it is possible to attach F-actin via electrostatic interaction to a lipid bilayer doped with positive charged lipids. [116] Three different receptor lipids were tested: DOEPC, a positively charged derivate of DOPC, DC-Cholesterol (DC-Chol), a positively charged derivate of cholesterol, and DOTAP, a synthetic lipid with the same fatty acid composition as DOPC (detailed information in chapter 3.1.1). Indentation measurements using an atomic force microscope (AFM) as described in chapter 3.5.1.1 were performed using an Asylum Research MFP-3D classic AFM (Asylum Research, Santa Barbara, CA, USA) or a JPK Nanowizard 4 (JPK Instruments, Berlin, Germany). Composition of the lipid bilayer was tuned to get effective F-actin binding and simultaneously stable PSLBs with a diameter of $1.2 \mu \mathrm{m}$. For effective F-actin binding a preferably high ratio of positively charged lipid is desired. Only DOTAP showed spreading of long-term stable PSLBs as shown in Figure 4.34 . 

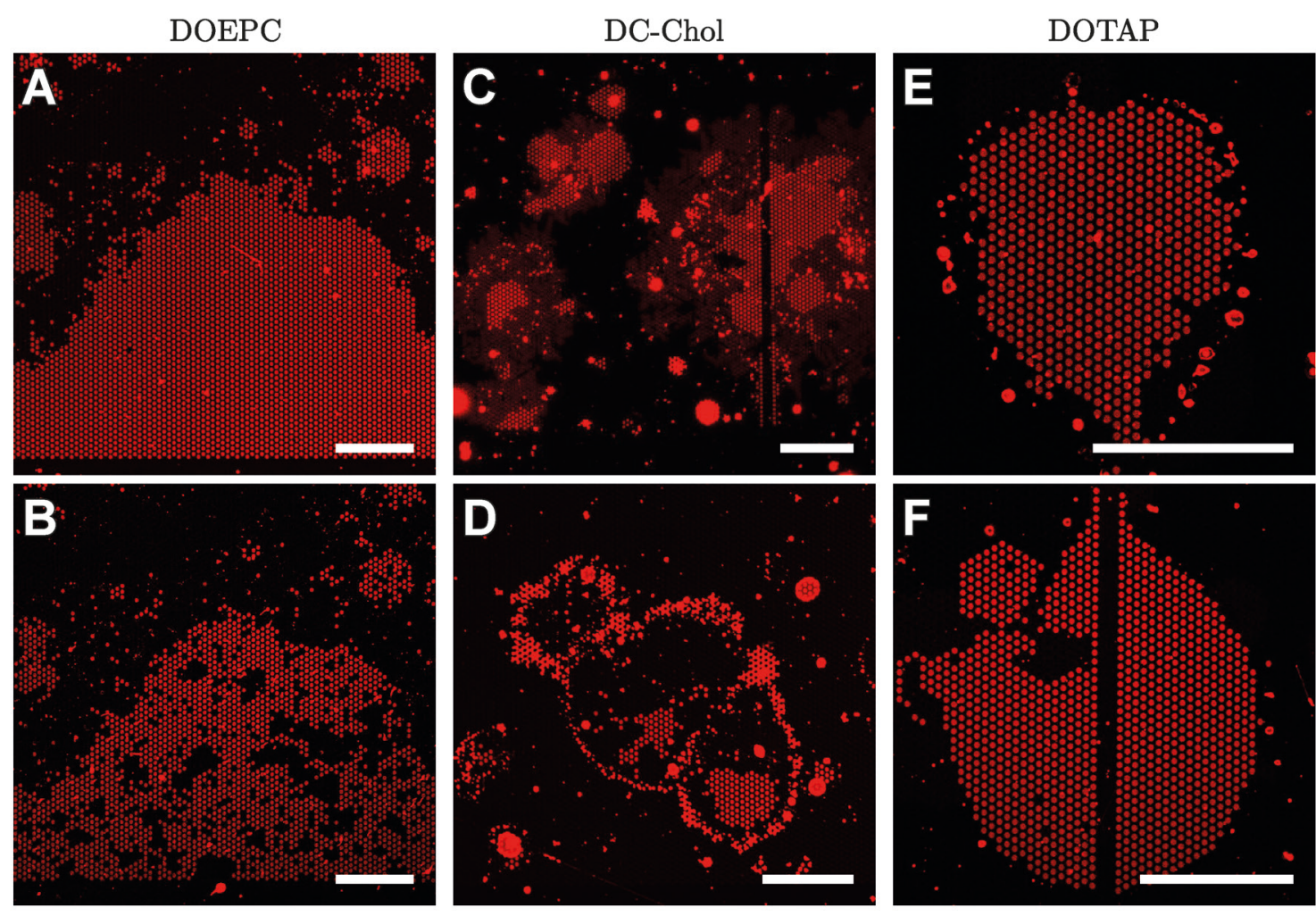

Figure 4.34: Differences in spreading behavior and stability of different positively charged receptor lipids. A-B: DOPC/DOEPC/TxR-DHPE (79.6:20:0.4) produced stable PSLBs $(\varnothing=1.2 \mu \mathrm{m})$ at first glance $(\mathbf{A})$ but the GUV patch was not stable over a duration of 6 hours (B). Almost half of the PSLBs ruptured within this time. C-D: In case of DC-Chol, sufficient PSLB could be formed using $10 \mathrm{~mol} \%$ DC-Chol. While this composition barely formed many PSLBs (C), a higher content of $20 \mathrm{~mol} \%$ led apparently to bursting of GUVs (D). E-F: Using the lipid DOTAP successful spread GUV patches with a content of $20 \mathrm{~mol} \%$ could be observed. The PSLBs were also stable overnight without increased incidence of PSLB rupture $(\mathbf{F})$. Scale bars: $30 \mu \mathrm{m}$.

Fluorescence micrographs shown in Figure 4.34 present red fluorescent, circular PSLBs in a hexagonal array, which derives from the porous substrate underneath. The fluorescence on the pore rims is quenched due to the gold functionalization (Figure 4.34 A). ${ }^{[236]}$ Using DOEPC in a ratio of $20 \mathrm{~mol} \%$, many stable PSLBs were observed at first sight. These PSLBs were apparently only stable for a few hours, because circular PSLBs vanished during imaging by means of confocal laser scanning microscopy (Figure $4.34 \mathrm{~B}$ ) indicating a rupture of these PSMs. In case of DC-Chol, the amount of obtained PSLBs was highly dependent on the molar ratio of receptor lipid. Using $10 \mathrm{~mol} \%$ DC-Chol patches with many PSLBs were obtained (Figure $4.34 \mathrm{C}$ ), while a higher content of $20 \mathrm{~mol} \%$ DC-Chol showed only a few PSLBs (Figure $4.34 \mathrm{E}$ ) which are orbited by round structures with a high fluorescence signal. This can be assigned to bulky lipid material that appears around the PSLB-array, indicating the whole, initial spreading GUV patch (Figure 4.34 D). This fluorescent signal was found to be on the surface of the substrate, not covering the pores. This obvious presence on the solid 
support emphasizes a large height because the fluorescence is not quenched by the gold functionalization. The observation of stacked up lipid material on the supported parts of porous substrates has been reported in literature and is described as effect of the violence of vesicle spreading.[233] Considering these intensities are stacked up lipid material, the hypothesis is made that the higher content of DC-Chol led to a higher violence or a more rapid vesicle spreading process and therefore only a few pores could be spanned (Figure $4.34 \mathrm{D}$ ) and most of the lipid material stacked up around the spreading area. Both problems of stability and spreading success did not occur in case of spreading vesicles containing 20 mol \% DOTAP which led to many PSLBs formed by a spread GUV patch (Figure $4.34 \mathrm{E}$ ). These PSLBs were also stable overnight (Figure $4.34 \mathrm{~F}$ ).

Thus, the study of F-actin induced change on the mechanical properties of lipid bilayers was conducted using DOTAP because it appeared to be the most suitable positively charged lipid. The following indentation experiments were carried out by Peter Mühlenbrock as part of his master thesis. It was already shown in literature that vesicles doped with DOTAP bind actin strongly, even upon applying an external force. ${ }^{[103]}$ Before indentation experiments were pursued, the PSLBs and further on the attached minimal actin cortices (MACs) were characterized by confocal fluorescence micrographs (Figure 4.35).
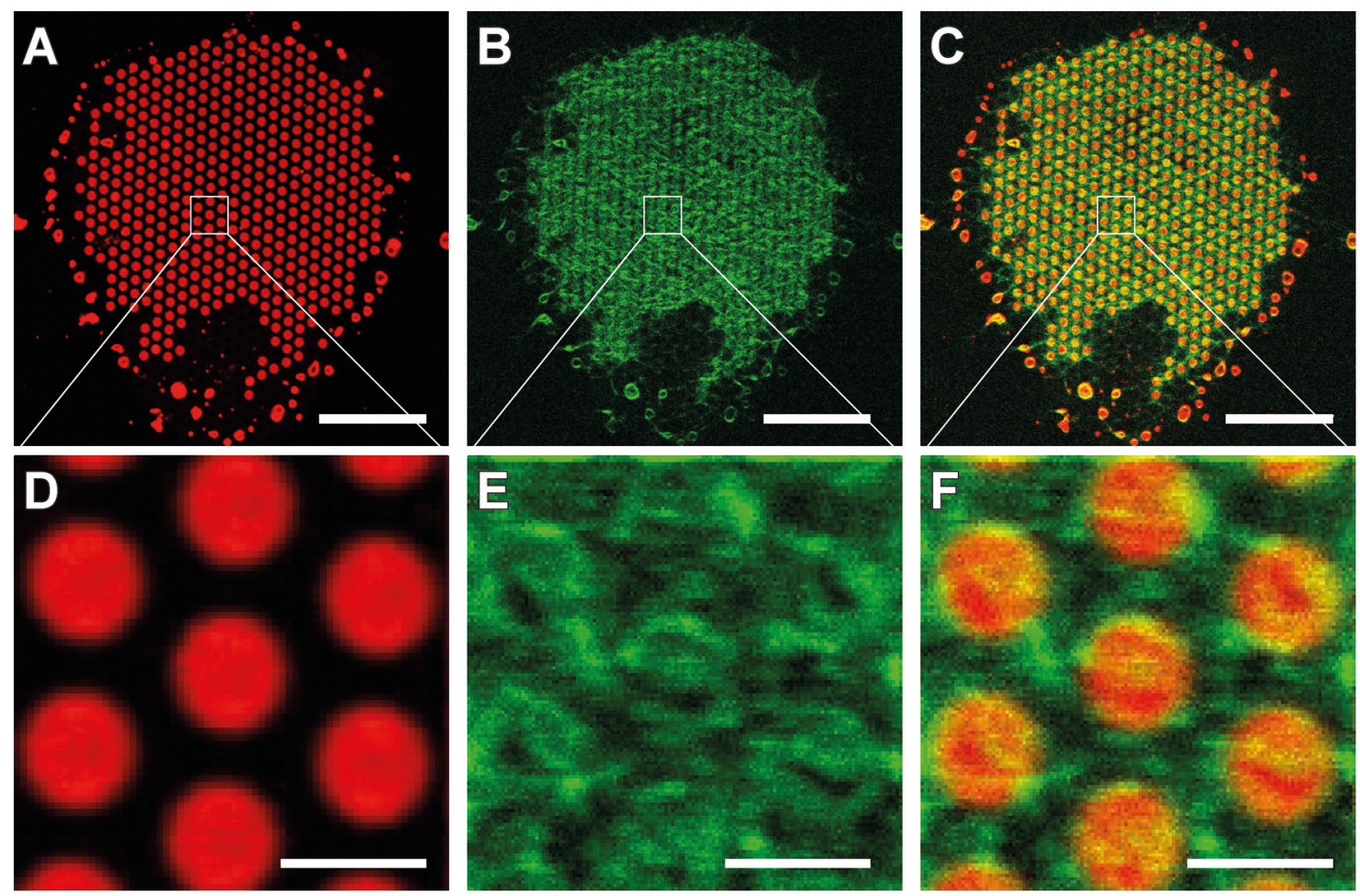

Figure 4.35: Successfully attached MACs to PSLBs (DOPC/DOTAP/TxR-DHPE 79.6:20:0.4). A: A GUV is spread on a porous substrate $(\varnothing=1.2 \mu \mathrm{m})$. PSMs can be recognized as circular TxR-DHPE fluorescent signal (red) of the lipid bilayer while the supported lipid bilayer parts are quenched by the gold functionalization. The border of the whole spread GUV is visible as bulky lipid material, indicated by the higher intensity, around 
the patch. A magnification (D) shows the hexagonal arrangement of the substrate with quenched parts between round PSLBs. B: The corresponding fluorescence signal of Alexa Fluor ${ }^{\circledR}$ 488-phalloidin bound to F-actin is shown in green. Short filamentous structures are visible over the whole patch. A magnification $(\mathbf{E})$ reveals bend and straight filaments attached to PSLBs as well as to the solid supported parts. C/F: An overlay of both fluorescence signals reveals a clear co-localization of F-actin and lipid bilayer. Scale bars: A-C: $15 \mu \mathrm{m}, \mathrm{D}-\mathrm{F}$ : $1.5 \mu \mathrm{m}$.

These micrographs demonstrate stable PSLBs as already shown in Figure $4.34 \mathrm{C} / \mathrm{F}$, and they reveal a specific F-actin attachment to these spread GUV patches (Figure 4.35). The resulting MAC (Figure $4.35 \mathrm{~B}$ ) covers the whole GUV patch (Figure 4.35 C). Magnifications of the MAC structure (Figure 4.35 D-F) show that F-actin filaments can be found on the solid supported membrane parts as well as on the pore spanning lipid bilayers. Having regard only to the Alexa Fluor ${ }^{\circledR}$ 488-phalloidin fluorescence signal obtained from F-actin filaments (Figure $4.35 \mathrm{E}$ ), one can already guess the position of the PSLBs as the filaments bend and trace the pore rim. The F-actin attachment is restricted to the GUV patch because the substrate was passivated with bovine serum albumin (BSA) after PSLB formation, which is necessary to avoid F-actin binding to the remaining free substrate parts.

Among the experiments the observed morphology of the MACs differed significantly, even within one preparation. Different GUV patches on a single substrate showed varying structures of bound F-actin filaments. In addition to the morphology displayed in Figure 4.35, many further morphologies were found. In Figure 4.36 exemplary fluorescence micrographs of different network morphologies are shown. 

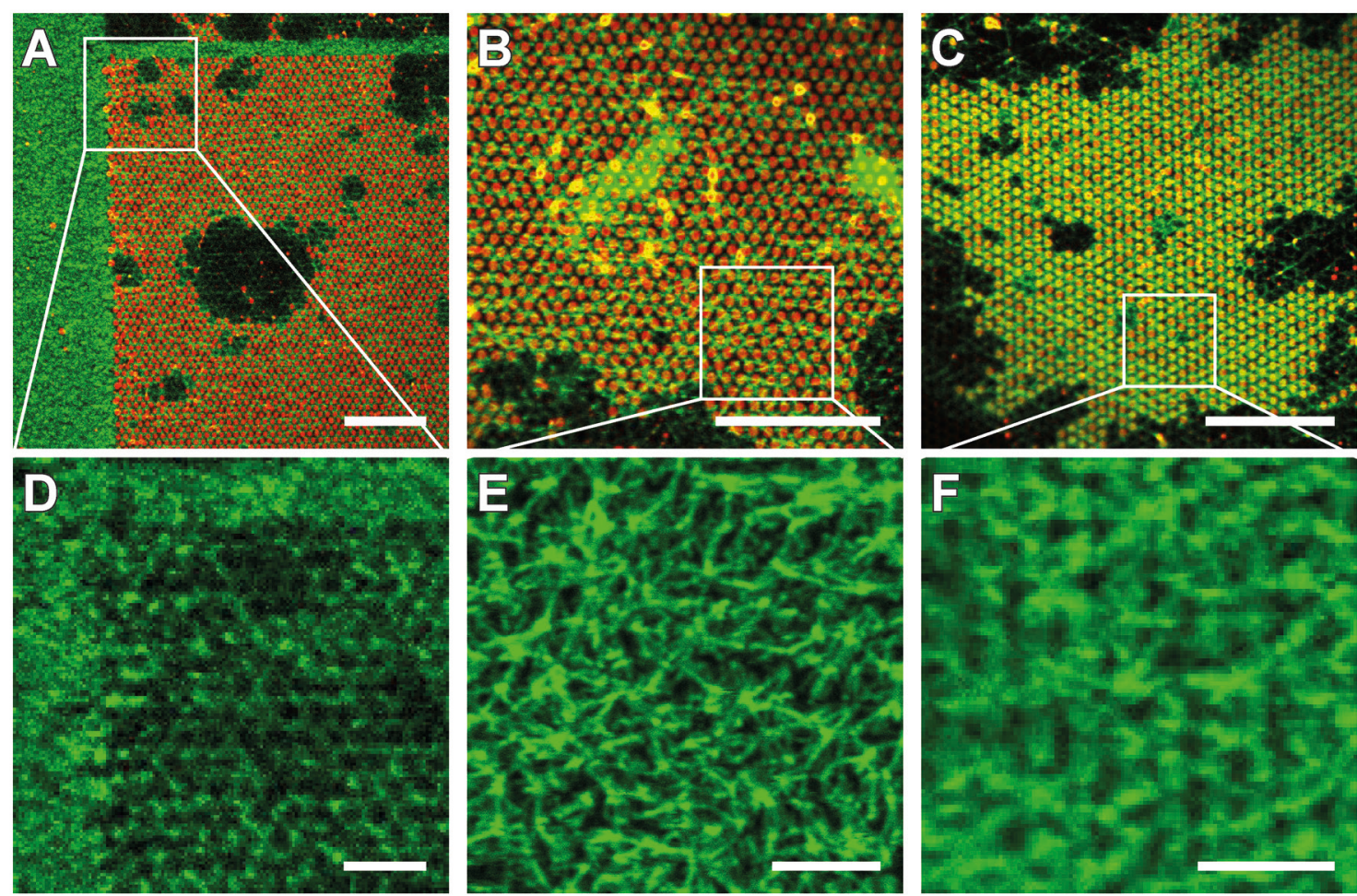

Figure 4.36: Different F-actin morphologies attached to PSLB with a pore diameter of $1.2 \mathrm{\mu m}$. A-C: Different GUV patches revealing PSLBs (red, TxR-DHPE) and attached MAC (green, Alexa Fluor ${ }^{\circledR}$ 488-phalloidin). D-F: Corresponding magnifications. D: In this example the attached F-actin seems to bind preferably to the solid supported lipid bilayer parts of the GUV patch. E: The morphology of some F-actin networks resemble MACs on solid supported membranes. F: A network morphology was observed which is similar to the morphology presented in Figure 4.35, but in contrast to these networks they show an additional almost circular fluorescence signal on the PSLBs. Scale bars: A-C: $20 \mu \mathrm{m}, \mathrm{D}-\mathrm{F}: 5 \mu \mathrm{m}$.

While Figure 4.35 displays F-actin networks with no preferred binding to one part of the substrate, the observed network shown in Figure $4.36 \mathrm{~A}$ has an obvious preference for binding to the substrate supported lipid bilayer parts. The circular PSLB areas can be observed as black, fluorescence free areas in the Alexa Fluor $488^{\circledR}$ fluorescence micrograph (cf. Figure 4.36 D) In contrast to that, F-actin networks were found which demonstrate clear and straight filaments without a preference for any substrate part (Figure 4.36 B). Compared to the morphology in Figure 4.35, the filaments do not show a circular curvature depending the underlying PSLB. In the morphology of this F-actin network (Figure $4.36 \mathrm{E}$ ) no indication to the underlying PSLB array can be found. In Figure $4.36 \mathrm{~F}$, a network morphology is shown which also displays filamentous structures with no preference to one part of the lipid bilayer, but this morphology (cf. Figure $4.36 \mathrm{~F}$ ) additionally show an almost circular fluorescence signal directly on the PSLBs. From all experiments $(N=34)$ it can be concluded that the networks preferentially bind to the solid supported parts of the lipid bilayer. 
Additionally, F-actin network morphologies were investigated on pores with a diameter of $5.0 \mathrm{\mu m}$. Keeping all other experimental conditions of GUV composition and substrate treatment the same, the same differences in network morphologies were observed on these porous substrates. An exemplary fluorescence micrograph and its magnification are displayed in Figure 4.37.
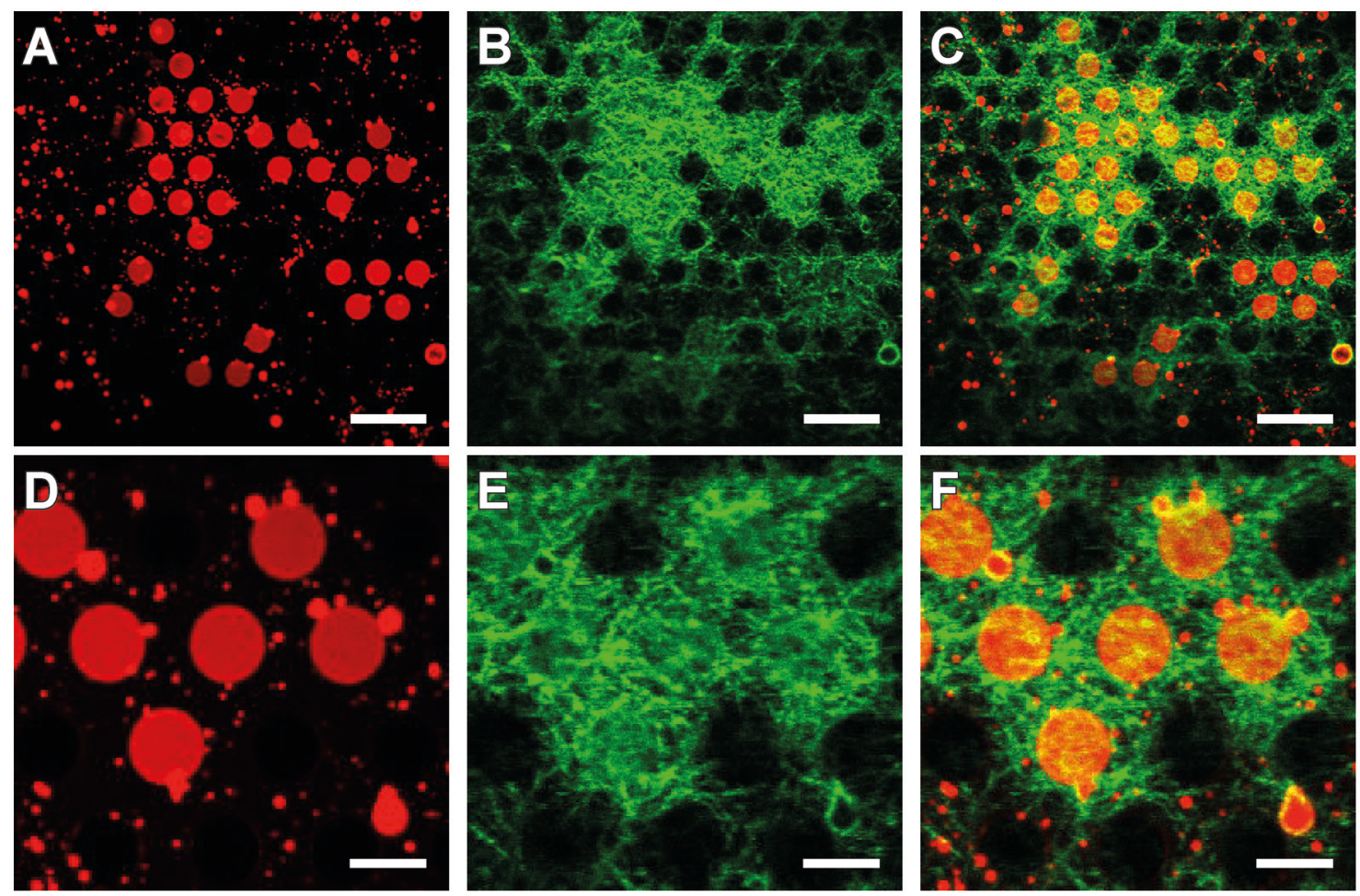

Figure 4.37: Alexa Fluor ${ }^{\circledR}$ 488-phalloidin labeled MACs attached to PSLBs (DOPC/DOTAP/TxR-DHPE (79.6:20:0.4) with a pore diameter of $5.0 \mathrm{\mu m}$. A-B: TxR-DHPE fluorescence signal reveals PSLBs and lipid material lying on the pore rims. C-D: Alexa Fluor ${ }^{\circledR} 488$ fluorescence signal shows the attachment of F-actin structures to PSLBs and the solid supported parts of the lipid bilayer. D-F: An overlay of both fluorescence signals shows significant binding to the PSMs. Open pores, suggested by the hexagonal array, display no fluorescence. Scale bar: A/C/E: $15 \mu \mathrm{m}, \mathrm{B} / \mathrm{D} / \mathrm{F}: 5 \mu \mathrm{m}$.

In Figure 4.37 a successful spreading of GUVs (A-B) as well as an attached F-actin network is shown (C-D). The MAC attached to free-standing parts of the lipid bilayer as well as to the solid supported parts (Figure 4.37 E/F). At first glance, the network morphology appears to have no preference of binding either the free-standing or the solid supported parts of the lipid bilayer.

Again, a number of different network morphologies were found, similar to the observations on $1.2 \mu \mathrm{m}$ PSLBs (Figure 4.38). In a recurring motif, a clear preference of F-actin binding to the solid supported parts of the lipid bilayer was found (Figure $4.38 \mathrm{D})$. However, filamentous structures also appeared on the free-standing parts of the lipid bilayer, as unique morphologies only found on PSLBs with a diameter of $5 \mu \mathrm{m}$ (Figure $4.38 \mathrm{E}$ ). These filaments bend as it seems like the structure is tracing the pore 
rims and will not protrude out of the circular PSLB. Besides these examples, also filamentous structures with an underlying blurry fluorescence signal only on the PSLBs were observed (Figure 4.38 F) as it was already found for PSLBs with a diameter of $1.2 \mu \mathrm{m}$. Among the recorded fluorescence micrographs on $5 \mu \mathrm{m}$ pores $(N=18)$ again a slightly higher probability of occurrence of F-actin networks preferably binding to the solid supported parts of the lipid bilayer as shown in Figure $4.38 \mathrm{~A} / \mathrm{D}$ was observed.
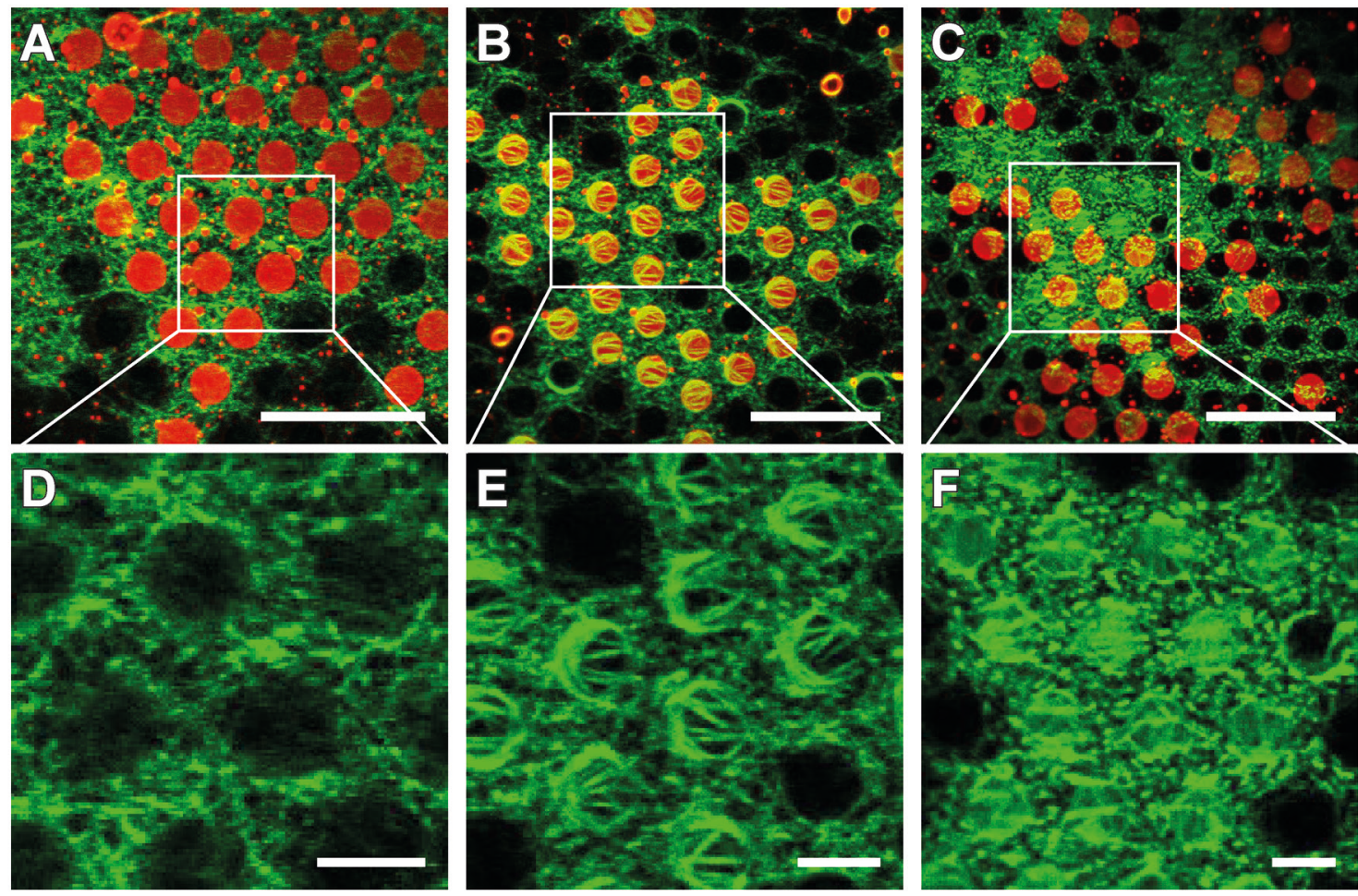

Figure 4.38: Morphologies of found F-actin networks attached to PSLB with a pore diameter of $5.0 \mathrm{pm}$. A-C: An overlay of PSLB fluorescence signal (red, TxR) and phalloidin labeled F-actin fluorescence (green, Alexa Fluor ${ }^{\circledR} 488$ ) reveals different network morphologies. D-F: Corresponding magnifications D: The network binds preferably on the pore rims. E: Long filaments are observed directly on the PSLBs. They bend at the pore rims to stay at the PSLB. F: Additional to observed filaments, a blurry fluorescence signal on top of the PSLBs is detected. Scale bars: A-C: $20 \mu \mathrm{m}, \mathrm{D}-\mathrm{F}$ : $5 \mu \mathrm{m}$.

\section{Height Difference of Pore Rim and Pore Center}

In some fluorescence micrographs of the lipid bilayers the pore rims were slightly highlighted in fluorescence signal, which is best to see in Figure 4.37 D. These donut shapes led to the assumption that the lipid bilayer is inclines into the pore. Especially with changing the pore diameter the parameter of height difference gets important, also considering the possibility of F-actin attachment to the PSLB. For this purpose, forceindentation experiments of the pore rim and the corresponding pore center were recorded. Care was taken to ensure that both corresponding points were on one horizontal or vertical line deriving from the topographical QI image to avoid a possible impact of an inclined position of the substrate. The trigger point was set to $0.5 \mathrm{pN}$ and 
in contrast to usual force-distance curves the contact point of cantilever tip and the PSLB was not leveled to zero. Thus, both determined curves were comparable, and the resulting height difference was evaluated (cf. Figure 4.39).

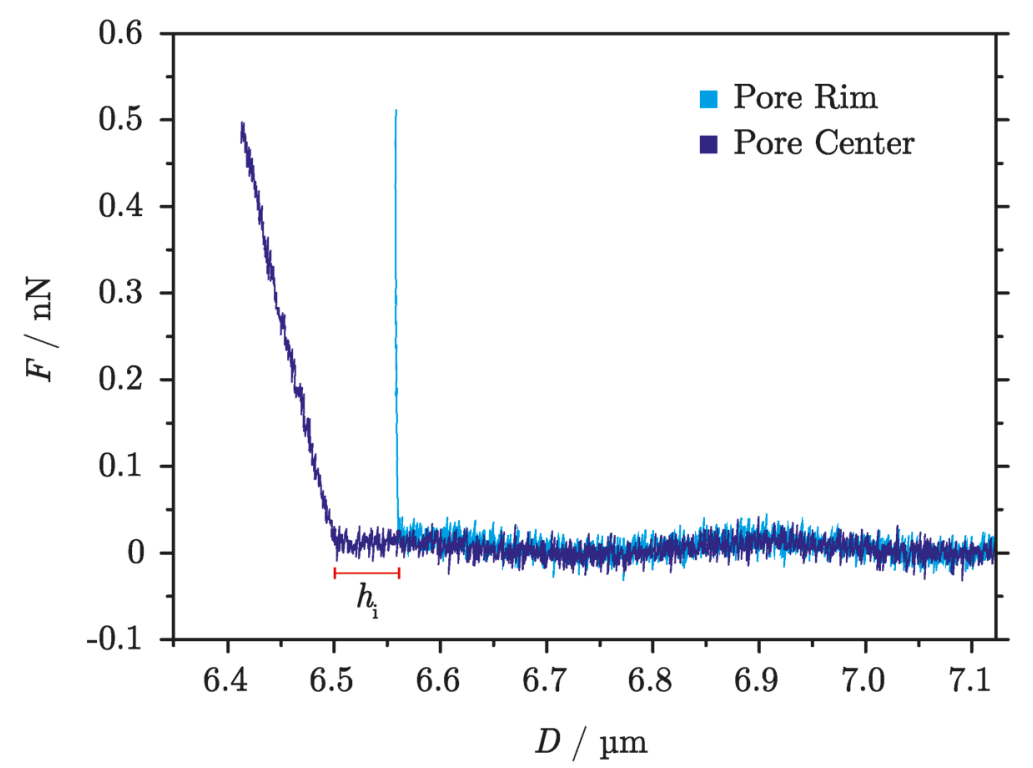

Figure 4.39: Exemplary force-distance curves, not leveled to the contact point, reveal a height difference between pore center and pore $\operatorname{rim} \boldsymbol{h}_{\mathbf{i}}$. One example of 109 comparisons of a force-distance curve on the pore rim (light blue) with a force-distance curve in the pore center (dark blue) shows a distinct height difference $h_{1}$. This example was recorded on a PSLB with a diameter of $1.2 \mu \mathrm{m}$ and an attached MAC.

In total, 109 relating force-distance curves of the pore rim (Figure 4.39, light blue) with the pore center (Figure 4.39, dark blue) were conducted. The height difference was determined as illustrated in Figure 4.39 (red line). The dependence of pore size and MAC attachment was investigated, and the collected data is shown in Table 4.7. All obtained height differences were in the same range within error margins. Therefore, no dependency of height difference and pore size was determined. Interestingly, all observed values were only slightly higher than the height of the evaporated gold layer. It might be assumed that the lipid bilayer leads along the edge of the gold layer till it loses the contact to the functionalization of the substrate. ${ }^{[234,237]}$

Table 4.7: Height differences $h_{\mathrm{i}}$ between pore rim and pore center in dependency of pore size and attached MAC.

\begin{tabular}{c|r|cc}
$\varnothing$ Pore Size / $\mathbf{~ m}$ & \multicolumn{2}{|c|}{$\boldsymbol{h}_{\mathbf{i}}$ (w/o MAC) / nm } & \multicolumn{2}{|c}{$\boldsymbol{h}_{\mathbf{i}}(\mathbf{w} \mathbf{M A C}) / \mathbf{n m}$} \\
\hline $\mathbf{1 . 2}$ & $45 \pm 10 \quad(N=10)$ & $60 \pm 15 \quad(N=22)$ \\
$\mathbf{5 . 0}$ & $50 \pm 40 \quad(N=40)$ & $30 \pm 20 \quad(N=37)$
\end{tabular}




\section{Influence of BSA Passivation}

The functionalized substrate surface was passivated with BSA after PSLB formation to prevent F-actin binding to these bilayer-free parts. Thus, a possible influence of BSA to the positively charged lipids as well as an influence on the subsequent indentation experiments had to be proven. In RIfS experiments (cf. chapter 3.4.1 and 4.1.3) the change in optical thickness $(\triangle O T)$ for DOPC/DOTAP (8:2) spreading and BSA incubation were measured in a time resolved manner. The composition of the lipid bilayer was identical to the GUV suspension used for PSM formation, lacking the fluorophore. An exemplary RIfS diagram is shown in Figure 4.40.

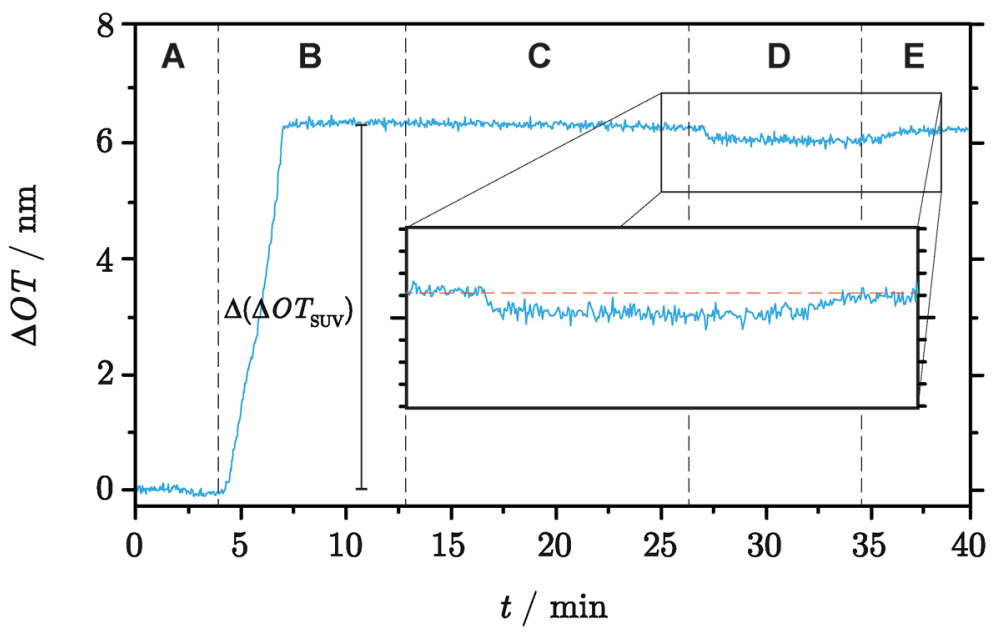

Figure 4.40: Exemplary RIfS diagram of spreading DOPC/DOTAP (8:2) SUVs and subsequent incubation with BSA. RIfS measurements were employed as described in chapter 3.4.1. After a baseline was recorded in PBS (A), spreading of SUVs, consisting of DOPC/DOTAP (8:2), in PBS was measured (B). The change in $O T$ was determined to $(6.4 \pm 0.3) \mathrm{nm}(N=4)$. Subsequent rinsing $(\mathbf{C})$ was followed by BSA incubation $(5 \mu \mathrm{M}$ in PBS, D) visible as slight decrease in $O T$ of $(0.3 \pm 0.1) \mathrm{nm}$. Rinsing with PBS changed the optical thickness back to the plateau level before BSA incubation $(\mathbf{E})$.

RIfS measurements showed a clear change in $O T$ for spreading of SUVs consisting of DOPC/DOTAP (8:2). The change in $O T$ was determined to $\Delta\left(\Delta O T_{\mathrm{SUV}}\right)=(6.4 \pm 0.3) \mathrm{nm}$ which is close to the detected change in $O T$ of POPC/PtdIns $[4,5] \mathrm{P}_{2}$ SUVs $((6.8 \pm 0.6) \mathrm{nm}$, cf. chapter 4.1.3), indicating a successfully spread lipid bilayer. The subsequent incubation with BSA appears to show no significant binding as rinsing with PBS after BSA incubation displays the same $O T$ as the spread lipid bilayer before. During BSA incubation the optical thickness is slightly decreased (Figure $4.40 \mathrm{D}$ ). A possible origin for this slight decrease can be a buffer effect. That a slight decrease is detected can originate from protein in solution, considering that the refractive index of most proteins is between 1.36 and $1.55,{ }^{[127]}$ while the refractive index of $\mathrm{BSA}$ is significantly higher $\left(n_{\mathrm{BSA}}=1.9\right) .{ }^{[238,239]} \mathrm{A}$ relatively 
higher refractive index affecting the protein-buffer solution could maybe lead to a slightly decreased $O T$ at the same level of physical thickness. Accordingly, a binding of BSA to positively charged lipid bilayers was excluded and subsequent rinsing with PBS (Figure $4.40 \mathrm{E}$ ) verified an available charged PSLB as the optical thickness got to the same plateau level as before BSA incubation.

Although there was no effect in BSA binding to positively charged lipid bilayers, it was checked if BSA passivation had an influence on the indentation experiments. For this, indentation experiments were performed directly before and after 1 hour of BSA passivation on one substrate. Before indentation, specimens were rinsed with buffer. Furthermore, a possible dependency on the trigger point to the slope $\left(k_{\text {app }}\right)$ of force-indentation curves was checked. One single PSLB was therefore indented three times, either with rising trigger point from $0.2 \mathrm{nN}$ via $0.5 \mathrm{nN}$ to $1.0 \mathrm{nN}$ or descending trigger point from $1.0 \mathrm{nN}$ via $0.5 \mathrm{nN}$ to $0.2 \mathrm{nN}$. A typical force-indentation curve and the resulting values are displayed in Figure 4.41. Noteworthy, only the resulting indentation of the first $200 \mathrm{pN}$ were fitted for determination of the slope.
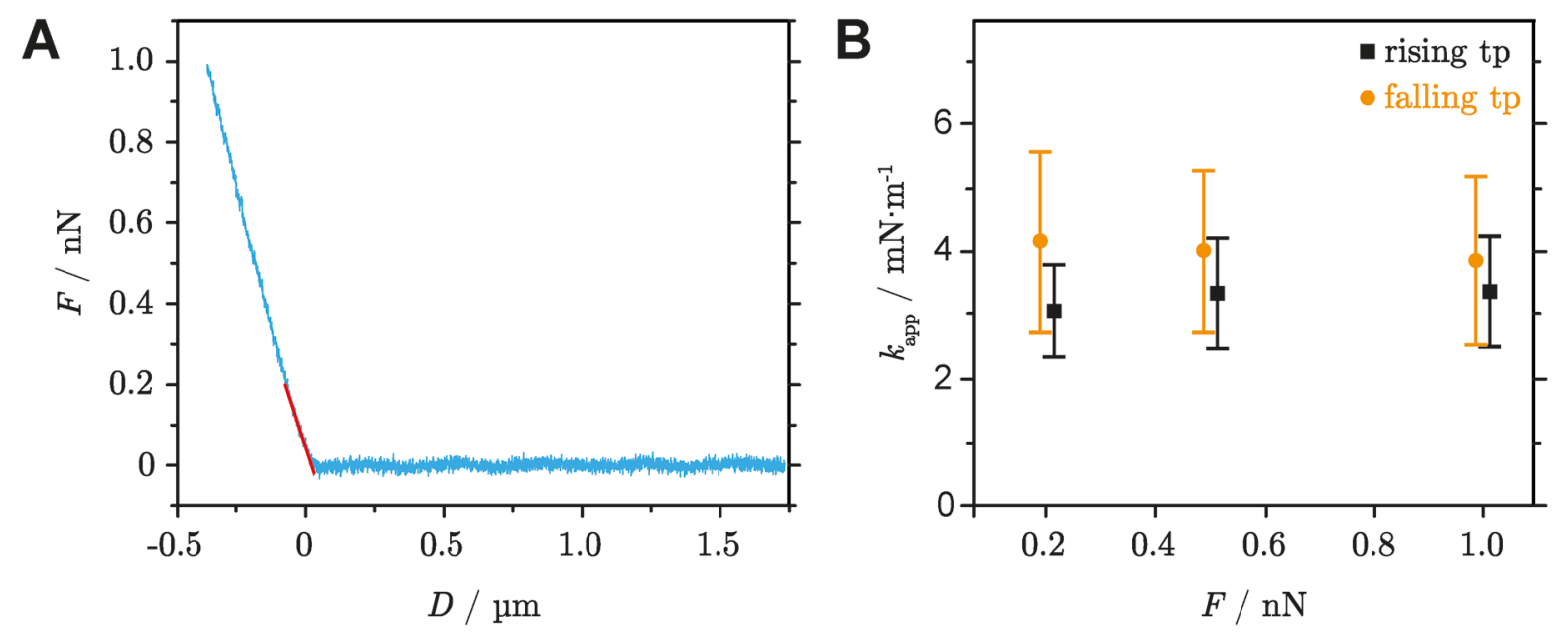

Figure 4.41: Representation of a typical force-distance curve and the resulting slopes $\left(k_{\text {app }}\right)$ of a PSLB (incubated with BSA) indentation experiment with a trigger point of $1 \mathbf{n N}$. A: A typical force-distance curve (FDC, trace) is shown. The contact point is set to zero. The curve is non-linear, only the first $0.2 \mathrm{nN}$ of indentation were evaluated (red line) to obtain the slope $\left(k_{\text {app }}\right)$. B: Single PSLB were indented three times either with rising trigger point (tp, black, $N=18$ ) or falling trigger point (orange, $N=28$ ). Data points were slightly displaced for reasons of clarity. The slope remains unaffected by varying the trigger point. The error bars display the standard deviation of the mean value.

The indentation experiments of DOPC/DOTAP/TxR (79.6:20:0.4) bilayer after BSA incubation showed no dependence of the trigger point (cf. Figure $4.41 \mathrm{~B}$ ). Additionally, no change in the slope (apparent spring constant $k_{\mathrm{app}}$ ) in contrast to untreated PSLBs was detected (not shown). With no influence of BSA detected, indentation experiments characterizing the influence of MACs attached to PSLBs were performed. 


\section{Influence of MACs on the Apparent Spring Constants ( $\left.k_{\mathrm{app}}\right)$ of PSLBs}

Since the attachment was characterized successfully by means of confocal fluorescence microscopy and an effect of BSA incubation was excluded, indentation experiments were performed as described in chapter 3.5.1.1. The mechanical properties of PSLBs with a diameter of 1.2 and $5.0 \mu \mathrm{m}$ were characterized by evaluating a response force after indentation. For indentation a trigger point of $0.5 \mathrm{nN}$ was applied, (more than the required $0.2 \mathrm{nN}$ for evaluation) and the membrane response was measured with and without attached MAC.
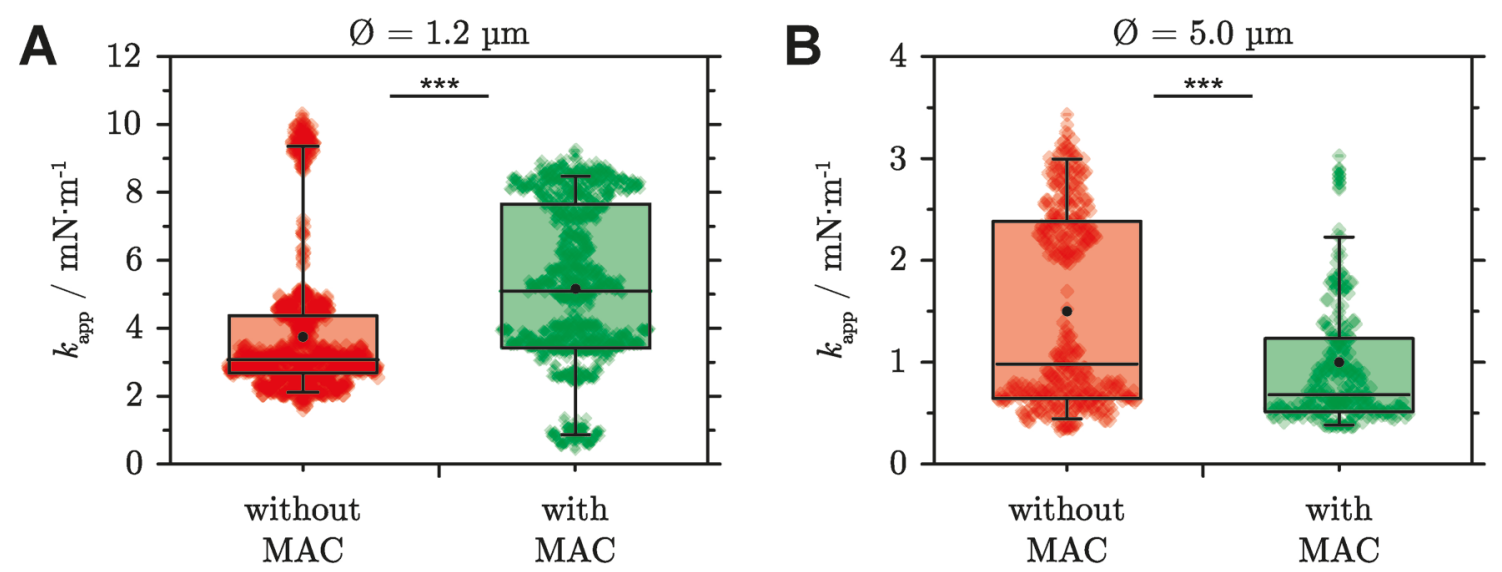

Figure 4.42: Boxplots displaying the apparent spring constants $\left(k_{\text {app }}\right)$ of PSLBs with different diameter as a function of an attached MAC. A: In case of PSLBs with a diameter of $1.2 \mu \mathrm{m} k$ app is determined to $(3.8 \pm 2.0) \mathrm{mN} \cdot \mathrm{m}^{-1}(M=6, N=1303)$. After formation of an MAC the apparent spring constant increases up to $(5.2 \pm 2.0) \mathrm{mN} \cdot \mathrm{m}^{-1}(M=4$, $N=1201)$. B: In case of PSLBs with a diameter of $5.0 \mu \mathrm{m} k_{\mathrm{app}}$ is determined to $(1.5 \pm 0.9) \mathrm{mN} \cdot \mathrm{m}^{-1} \quad(M=7, \quad N=438)$ without $\mathrm{MAC}$ and $(1.0 \pm 0.6) \mathrm{mN} \cdot \mathrm{m}^{-1} \quad(M=10$ $N=198$ ) with MAC. For analysis $M$ independent experiments (porous substrate) with $N$ force-distance curves were evaluated. Each data point is plotted in the background. Box plots represent data from $25^{\text {th }}$ to $75^{\text {th }}$ percentile, solid line displays the median and whiskers extent to the $5^{\text {th }}$ and $95^{\text {th }}$ percentile. A two sample Mann-Whitney $U$-test was performed. The null hypothesis was rejected in both cases at the $0.1 \%$ significance level $(* * *)$.

Figure 4.42 A displays the evaluated apparent spring constants $\left(k_{\mathrm{app}}\right)$ of 1303 force-distance curves $(N)$ out of 6 independent substrate preparations $(M)$ with a PSLB diameter of $1.2 \mu \mathrm{m}$. In case of these PSLBs a $k_{\text {app }}$ of $(3.8 \pm 2.0) \mathrm{mN} \cdot \mathrm{m}^{-1}$ was determined. This distribution of data points (Figure $4.42 \mathrm{~A}$, red) is nearly normally distributed with an outlier accumulation around $9.5 \mathrm{mN} \cdot \mathrm{m}^{-1}$. In literature kapp-values for PSLBs with the same diameter are reported, certainly of a purely DOPC bilayer. In these experiments a lower apparent spring constant of $k_{\text {app }}=(1.42 \pm 0.02) \mathrm{mN} \cdot \mathrm{m}^{-1}$ was obtained. [233] Furthermore, for pure DOTAP lipid bilayers an apparent spring constant of $k_{\mathrm{app}}=(3.90 \pm 0.08) \mathrm{mN} \cdot \mathrm{m}^{-1}$ was obtained on PSLBs with a diameter of $90 \mathrm{~nm}$ and a different functionalization. ${ }^{235]}$ Besides the different functionalization, this value is hard to compare as earlier studies revealed that the apparent spring constant increases 
with decreasing pore diameter. ${ }^{[139]}$ This dependence can be observed in the experiments of this work as PSLBs with a diameter of $5.0 \mu \mathrm{m}$ (Figure $4.42 \mathrm{~B}$, red) show a decreased apparent spring constant in contrast to PSLB with $\varnothing=1.2 \mu \mathrm{m}$. Besides the lower $k_{\text {app }}$ in contrast to PSLBs with $1.2 \mu \mathrm{m}$ diameter also two clear separated accumulations are observed. Resulting mean value of $k_{\text {app }}$ was determined to $(1.5 \pm 0.9) \mathrm{mN} \cdot \mathrm{m}^{-1}(M=7$, $N=438$ ). The lower apparent spring constant seems reasonable because the spring constant increases with decreasing pore diameter and vice versa. ${ }^{139]}$

After attaching F-actin to these PSLBs to form an MAC a significantly higher spring constant was observed in case of $\varnothing=1.2 \mu \mathrm{m}$. The mean value was determined to $(5.2 \pm 2.0) \mathrm{mN} \cdot \mathrm{m}^{-1}(M=4, N=1201)$. Interestingly, the data points reveal four accumulations (Figure $4.42 \mathrm{~A}$, green). Concerning MAC with a PSLB of $5 \mathrm{\mu m}$ pore diameter, the spring constant was decreased to $(1.0 \pm 0.6) \mathrm{mN} \cdot \mathrm{m}^{-1}(M=10, N=198)$, but also no normally distributed data was observed. In this case, one big accumulation of data points was detected around $9.5 \mathrm{mN} \cdot \mathrm{m}^{-1}$ and many data points spreading to higher values. On PSLBs with a diameter of $1.2 \mu \mathrm{m}$ the attachment of an MAC led to significantly increased apparent spring constants while on PSLBs with a diameter of $5.0 \mu \mathrm{m}$ the apparent spring constant decreased after attaching F-actin to form an MAC. Looking at the accumulations, it is conspicuous that these accumulations as well as different network morphologies were found. As the GUV patch for AFM measurements was monitored with an epifluorescence microscope, and not with a confocal laser scanning microscope, no correlation between network morphology and relating AFM indentation experiments could be established. It is assumed that different network morphologies lead to different $k_{\mathrm{app}}$-values. Nevertheless, to affirm this hypothesis different experimental days were plotted independently (cf. Figure 4.43). Since the preparation is very time-consuming only a small number of single GUV patches, which showed one special network morphology, were measured on one single experimental day. Therefore, the data was assorted to specific dates.
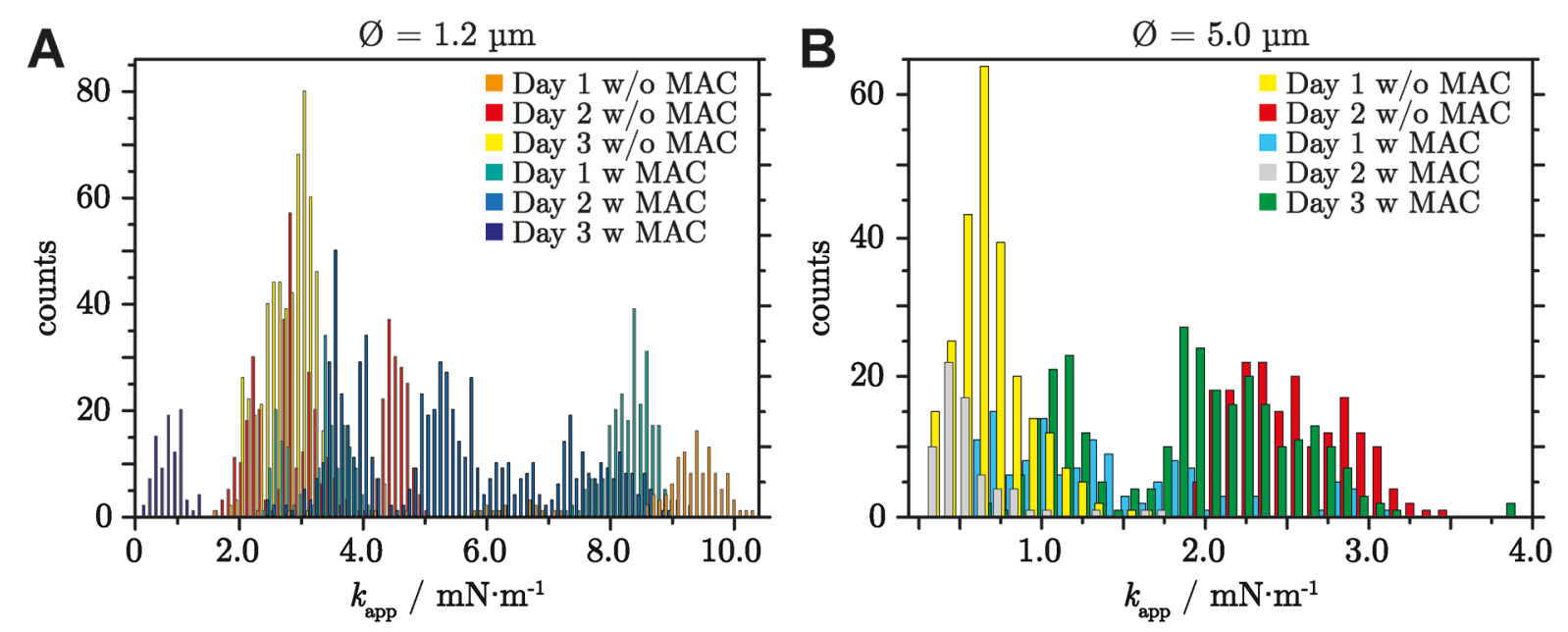

Figure 4.43: Correlation of determined apparent spring constants $\left(k_{\text {app }}\right)$ to different experimental days. With separating the spring constants in dependence to different 
experimental days conclusions to varying GUV patches and therefore different network morphologies can be drawn. A (1.2 $\mathbf{~ m})$ : Separate experimental days reveal that there is one outlier accumulation (orange) in the data of PSLBs without MAC while most data is spread around the mean value. Obviously, single experimental days show specific accumulations which are separately normally distributed. B $(\mathbf{5 . 0} \mathbf{~ H m})$ : Clearly visible, each experimental day without attached MAC led to a single accumulation of normally distributed data which does not fit to each other. With attached MAC two days (green and blue) led to distributed data over the whole regime of apparent spring constants while day 3 (green) led to two accumulations with different maxima. Interestingly, the mean value of day 2 without MAC appears to have a higher mean value than any other observed accumulations. Data was binned into bars of $0.1 \mathrm{mN} \cdot \mathrm{m}^{-1}$.

Figure 4.43 reveals a dependency of different experimental days to accumulations of apparent spring constants. A great spread within the separate accumulation is observed but is not restricted to measurements with attached MACs. In case of $1.2 \mu \mathrm{m}$ pores, the already detected outlier accumulation around $9.5 \mathrm{mN} \cdot \mathrm{m}^{-1}$ could be allocated to one single experimental day while remaining measurements are in a close range and appear to be almost normally distributed. The experimental day 2 which is displayed in red shows two separate accumulations around the accumulation of day 3 (Figure $4.43 \mathrm{~A}$, yellow). Nevertheless, the broad distribution of apparent spring constants is quite usual, because various articles in literature also report this phenomenon. [233,234,240] With regard to the measurements with attached MAC the already observed four accumulations could also be allocated to different experimental days. While the accumulation around $0.5 \mathrm{mN} \cdot \mathrm{m}^{-1}$ originates from one special experimental day (Figure $4.43 \mathrm{~A}$, day 3, dark blue), the remaining two days provide 2 accumulations in each case (day 1 , turquoise and day 2, blue). As exactly two turquoise and two blue accumulations superimpose each other only 4 independent accumulations are observed in the overall data.

An even more clear dependency was revealed within the data of $5.0 \mu \mathrm{m}$ pores. On one day, only low apparent spring constants under $1.5 \mathrm{mN} \cdot \mathrm{m}^{-1}$ could be determined (Figure $4.43 \mathrm{~B}$, grey) while on another day two accumulations (Figure $4.43 \mathrm{~B}$, green), one under, one above $1.5 \mathrm{mN} \cdot \mathrm{m}^{-1}$, were recorded. Only the dataset recorded on day 2 (cyan) appears to have no larger accumulation. Interestingly, already the datasets recorded without MAC separate into two accumulations (Figure $4.43 \mathrm{~B}$, red and yellow). While the dataset of day one (yellow) does not show any higher apparent spring constant than $1.75 \mathrm{mN} \cdot \mathrm{m}^{-1}$ the dataset of day 2 shows only apparent spring constants above $2 \mathrm{mN} \cdot \mathrm{m}^{-1}$. Furthermore, it was shown that already GUV patches without MAC show a similar heterogeneity. This finding implies that further studies need a direct correlation between F-actin network morphology and the determined apparent spring constant. A correlation of one GUV patch before and after attachment should be established. Only using this correlation, the clear impact of the F-actin attachment can be determined. The development of a correlative CLSM and AFM is necessary for continuation and clear insights in this part of the project. 


\section{Effect of the AFM Measurement on the F-actin Network}

Before indentation experiments were employed, a topographical image of the porous substrate was taken using the QI mode. This image (cf. Figure $4.44 \mathrm{C}$ ) shows the localization of all PSLBs and open pores and can be used to position the subsequent force-indentation curve in the pore center. A possible impact of the force indentation measurements was analyzed by means of fluorescence microscopy. Fluorescence micrographs were taken directly before and after the AFM measurement was applied. Imaging was conducted using a trigger point of $0.15 \mathrm{nN}$ and $0.5 \mathrm{nN}$ was used to record FDCs. A trigger point of $0.15 \mathrm{nN}$ was the lowest usable force to obtain information about the localization of the PSLBs. Characterization of the AFM measurements impact was conducted four times on both pore sizes. Exemplary micrographs before and after the force indentation experiment is shown in Figure 4.44, using PSLBs with a diameter of $5 \mu \mathrm{m}$.
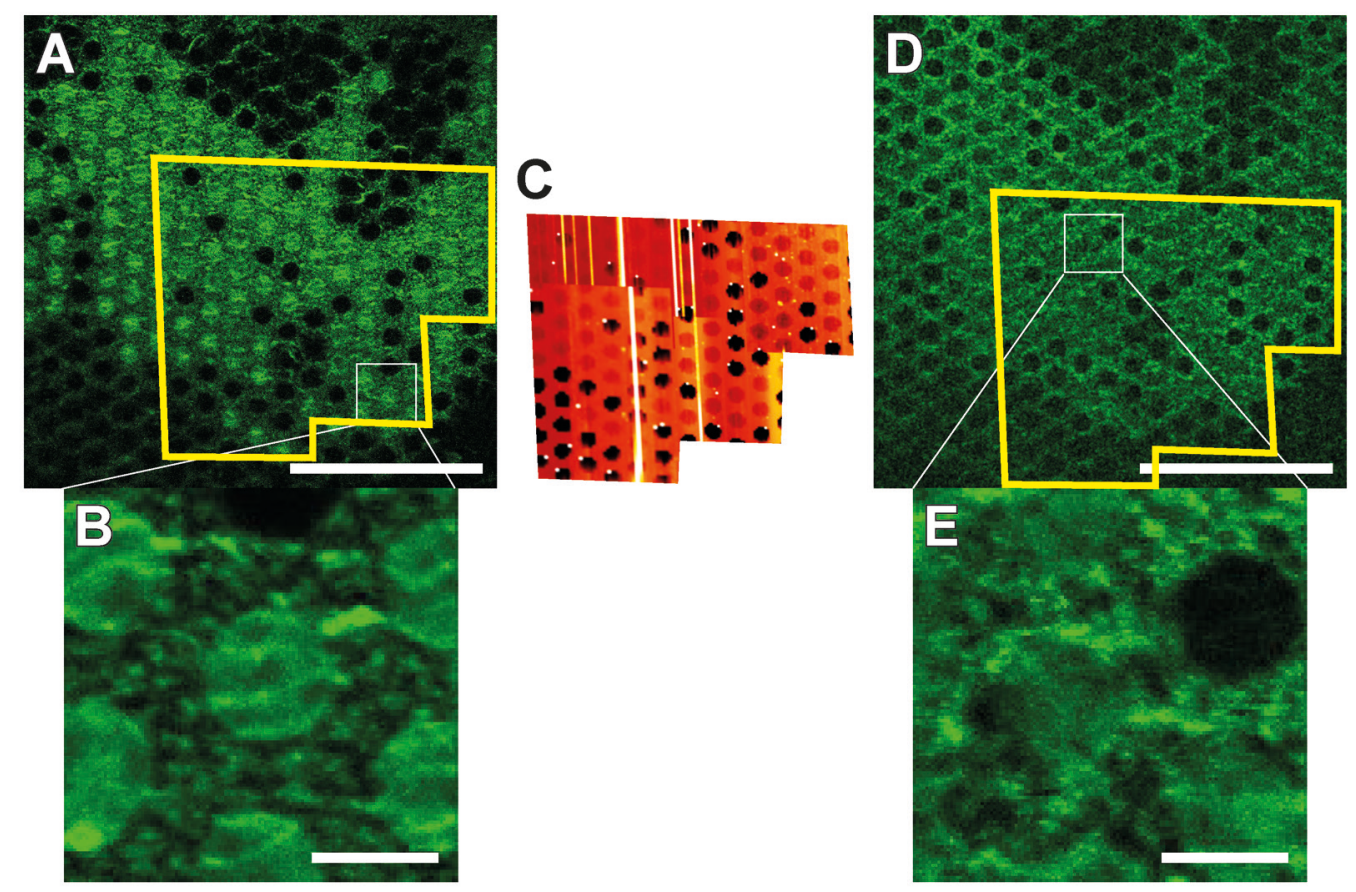

Figure 4.44: Influence of topographical AFM measurement on the morphology of attached F-actin networks revealed by fluorescence micrographs. A/B: Fluorescence micrograph showing the F-actin morphology of a network attached to PSLBs (DOPC/DOTAP/TxR-DHPE (79.6:20:0.4)) with a 5 um diameter. C: Subsequently, a few topographical AFM measurements using the QI mode were recorded. The resulting images were assembled which led to the unusual image shape. D/E: After AFM measurement the morphology of the F-actin network appears differently, especially directly on the PSLBs. Interestingly, the change in morphology does not restrict on the part of the image where the AFM measurement was applied (yellow boxes). Scale bars: A/D: $50 \mu \mathrm{m}$. B/E: 5 m.

These fluorescence micrographs (cf. Figure 4.44) show that a change in network morphology occurred during the time these fluorescence micrographs were taken. Obviously, there is still F-actin network present, before and after the applied QI mode measurement, but the morphology of the network appears to be less dense after the 
AFM measurements. Furthermore, it seems that the additional Alexa Fluor ${ }^{\circledR}$ 488-phalloidin fluorescence on the PSLBs vanishes (Figure 4.44, compare $\mathrm{B}$ and E). Interestingly, the changes are not limited to the area where the AFM measurement was applied (Figure 4.44 yellow boxes). This implies that the applied force of the cantilever may not be the only reason for the change in morphology. Another possible reason could be a slight temperature increase during the measurement induced by external factors like the lamp which is used to calibrate the AFM laser and tip. Fluorophore bleaching could also be considered but as the fluorophore is known to be photostable under the chosen conditions (e.g. Figure $4.44 \mathrm{~A}$ and B) it is not probable that exposition to slight ambient light can bleach the F-actin network to this extent. In conclusion, there is a slight change in F-actin morphology but the main reason for this change seems not to be the applied force by the cantilever. Relocating or total removal of the F-actin network by the cantilever can be excluded.

\section{Influence of MACs on the Lateral Membrane Tension of PSLBs}

The obtained apparent spring constant $k_{\text {app }}$ can depend on various physical properties: Bending, shearing, stretching and the lateral prestress of the planar membrane. As lipid bilayer are fluid, Evans et al. were able to show that shearing does not require energy and can therefore be neglected.[78] The remaining three contributions were estimated using interpretations deriving from Mey et al. ${ }^{[234]}$ Following these interpretations a rough estimation which contribution may dominate the mechanical properties of the lipid bilayer was made

They showed that the contribution of bending to the apparent spring constant can be calculated using equation 4.20 following the assumption that a small point load force is applied.[241] With the cantilever tip of $20 \mathrm{~nm}$ in relation to a pore diameter of 1.2 or $5.0 \mu \mathrm{m}$ this assumption is reasonable in the presented experiments.

$$
F(h)=\frac{64 \cdot \pi \cdot \kappa}{R_{\text {pore }}^{2} \cdot h}
$$

The bending modulus $\kappa$ of the lipid bilayer is estimated to $\kappa=1.1 \cdot 10^{-19} \mathrm{~J}$ by assuming reasonable values for the elastic modulus (Young's modulus) of $E=28 \mathrm{MPa}$, a Poisson ratio of $v_{P}=0.33$ and a membrane thickness of $d=5.5 \mathrm{~nm}$. ${ }^{[234]}$ With the pore radius $R_{\text {pore }}$ and the indentation depth $h$ bending contributions of $F_{1.2 \mu \mathrm{m}}=6.1 \cdot 10^{-5} \mathrm{~N} \cdot \mathrm{m}^{-1}$ and $F_{5.0 \mathrm{~mm}}=3.5 \cdot 10^{-6} \mathrm{~N} \cdot \mathrm{m}^{-1}$ were calculated. In the regime of detected $k_{\mathrm{app}}$ values of $1 \cdot 10^{-3} \mathrm{~N} \cdot \mathrm{m}^{-1}$ up to $6 \cdot 10^{-3} \mathrm{~N} \cdot \mathrm{m}^{-1}$ the contribution of bending is only a minor one and it can safely be assumed that bending is negligible.

The contribution of stretching has a high impact when applying stronger forces as the indentation depth is highly dependent on the applied force. ${ }^{[234]}$ The dependence of force 
to indentation depth for force-indentation curves can be calculated using a simple 2D Hooke's law (equation 4.21): [235,241-243]

$$
F(h)=g\left(v_{\mathrm{P}}\right) \cdot R_{\text {pore }} \cdot E \cdot d \cdot\left(\frac{h}{R_{\text {pore }}}\right)^{3}
$$

with $g\left(v_{\mathrm{P}}\right)=\pi / 3$ for $v_{\mathrm{P}}=0.33$. Interestingly, the obtained dependence is cubic which indicates that this contribution of stretching alone cannot explain the observed experimental force-distance curves which also corresponds with literature. ${ }^{[233,234]}$ Furthermore, lipid bilayers can only withstand an area dilatation of $3-5 \%$ and membrane rupture occurs if this is exceeded.[244,245] Assuming the cantilever tip to be conical, a calculation of the shell surface for the lipid bilayer can be performed and the area dilatation can be estimated. In the presented experiments even with the smallest indentation depth of $200 \mathrm{~nm}$ the area dilatation exceed $5 \%\left(5.4 \%, R_{\text {pore }}=600 \mathrm{~nm}\right.$, $h=200 \mathrm{~nm}$ ) which can only mean that lipid material flows into the indented PSLB. [234] The elastic response is described in detail by Norouzi et al. originating mainly from the deformation of a prestressed membrane. ${ }^{[246,247]}$ Theoretical studies neglecting bending of the lipid bilayer as argued above led to an analytical equation for using a small point tip concerning a homogeneous and constant prestress $\sigma .{ }^{[246,247]}$ These theoretical models predict a slight non-linearity which is congruent with the experiments in this chapter. Observed FDCs of this work support these models, because they were not completely linear, but a linear fit has to be applied concerning the continuum elasticity model. This theory can only be applied at small indentation depths which is the reason why only indentation until a trigger point of $0.2 \mathrm{nN}$ is reached will be evaluated.

$$
h=\frac{F}{4 \pi \cdot \sigma} \cdot\left(1-\ln \left(\frac{F \cdot R_{\text {tip }}}{2 \pi \cdot \sigma \cdot R_{\text {pore }}{ }^{2}}\right)\right)
$$

This almost linear behavior is also supported by molecular dynamic simulations. These simulations assumed indentations which result in forces between lipid bilayer and cantilever tip showing force-indentation experiments with a predominantly linear behavior. ${ }^{[248]}$ In total, this shows that the indentation must be dominated by the lateral prestress $\sigma$ of the membrane. This prestress or apparent lateral tension arises due to the difference in chemical potential between the indented lipid bilayer and the lipid reservoir on the pore rim. ${ }^{[234]}$ Due to the attractive forces between lipid headgroups and the hydrophilic functionalization of the SAM the lipids adhere to the pore rims and the PSLB is prestressed because of this adhesion. With equation 4.22 in hand the lateral membrane tensions can be calculated. The results are presented in Figure 4.45. 


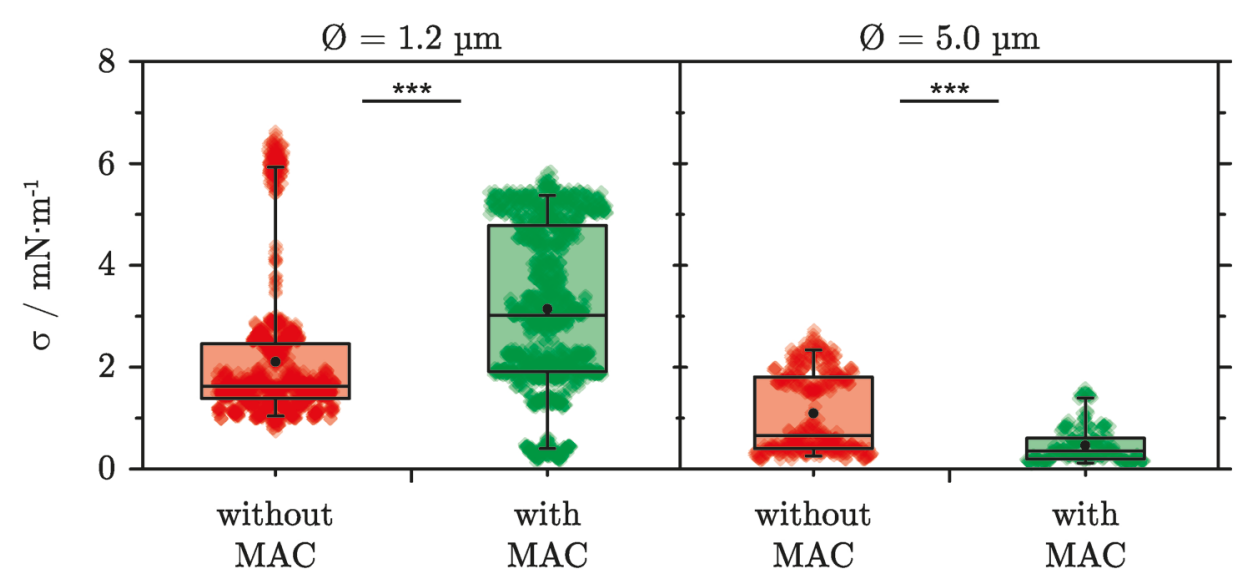

Figure 4.45: Boxplots displaying the lateral membrane tension $(\sigma)$ in both cases as a function of an attached MAC. In case of $1.2 \mu \mathrm{m}$ PSLBs in diameter a lateral membrane tension of $\sigma=(2.1 \pm 1.3) \mathrm{mN} \cdot \mathrm{m}^{-1}(N=1303)$ was determined. On the other hand, PSLBs with a diameter of $5.0 \mu \mathrm{m}$ reveal a lateral membrane tension of $\sigma=(1.1 \pm 0.8) \mathrm{mN} \cdot \mathrm{m}^{-1}(N=438)$. With an electrostatically bound MAC the tension increases up to of $\sigma=(3.2 \pm 1.3) \mathrm{mN} \cdot \mathrm{m}^{-1}$ $(N=1201)$ for PSLBs with $1.2 \mu \mathrm{m}$ and decreases to $\sigma=(0.5 \pm 0.4) \mathrm{mN} \cdot \mathrm{m}^{-1}(N=198)$ for PSLB with $5.0 \mu \mathrm{m}$. Box plots represent data from $25^{\text {th }}$ to $75^{\text {th }}$ percentile, solid line displays the median and whiskers extent to the $5^{\text {th }}$ and $95^{\text {th }}$ percentile. A two sample Mann-Whitney $U$-test was performed. The null hypothesis was rejected in both cases at the $0.1 \%$ significance level $(* * *)$.

The resulting lateral membrane tension of PSLBs with a diameter of $1.2 \mu \mathrm{m}$ was determined to be $\sigma=(2.1 \pm 1.3) \mathrm{mN} \cdot \mathrm{m}^{-1}$ (Figure 4.45). After an MAC was formed, the relating membrane tension increased to $\sigma=(3.2 \pm 1.3) \mathrm{mN} \cdot \mathrm{m}^{-1}$. In case of PSLBs with a larger diameter of $5.0 \mu \mathrm{m}$ a membrane tension of $\sigma=(1.1 \pm 0.8) \mathrm{mN} \cdot \mathrm{m}^{-1}$ without and $\sigma=(0.5 \pm 0.4) \mathrm{mN} \cdot \mathrm{m}^{-1}$ with attached MAC was found. Looking at equation 4.17, which considers the radius of the pore, it appears to be reasonable that the lateral membrane tensions for both pore sizes are in the same range, because the values should be independent from the pore size. However, the detected values differ slightly, but the values are the same within the error margin. This behavior is conclusive with findings in literature. ${ }^{[139]}$ Obviously, a large heterogeneity of $\sigma$ is found (Figure 4.45, compare red data points).

Concerning the MAC attachment, the impact of the MAC on the lateral membrane tension cannot be generalized as the tension increased with attached MAC in case of $1.2 \mu \mathrm{m}$ pores and decreased with attaching MACs on PSLB with a diameter of $5.0 \mu \mathrm{m}$. The fluorescence micrographs suggest that the morphology of the MACs which varies among single GUV patches, which appears to be the reason of the different behavior. On PSLBs with a diameter of $5.0 \mu \mathrm{m}$ larger F-actin structures were found which sometimes selectively prefer the PSMs instead of the solid supported lipid bilayer parts (cf. Figure 4.38 E). While the reason for this substrate dependent sorting remains unclear this general phenomenon of a sorting-effect during F-actin attachment was also 
observed on solid supported lipid bilayers (cf. chapter 4.2.2). It seems, that the larger the pore size gets, and therefore closer to the obtained in vitro filament length in $3 \mathrm{D}$ F-actin gels (cf. chapter 4.2.1), the longer the attached filaments get.[196,197] As already mentioned, a special type of network morphology could so far not be related to the lateral membrane tension. However, the observed dependencies indicate a possible correlation between network organization and elastic response of the lipid bilayer.

\subsubsection{Conclusion of Force-Indentation Experiments with Attached MACs}

For PSLBs with a diameter of $1.2 \mu \mathrm{m}$ a significant increase in lateral membrane tension upon MAC formation was observed similar to the findings of $C$. Kramer. ${ }^{[116]}$ The experimental setup was similar, because $1.2 \mu \mathrm{m}$ porous substrates were identically functionalized and positively charged lipids with a content of $20 \mathrm{~mol} \%$ were used. In these experiments the attachment of the F-actin network led to an increase in lateral membrane tension of $65 \%$. The detected increase in this work was determined to be around $50 \%$. In a top-down approach of Lorentz et al. basolateral membranes of Madin-Darby canine kidney (MDCK) II cells were attached on porous substrates, identical to the substrates with a diameter of $1.2 \mu \mathrm{m}$ used in this work. They investigated the effect of removing of the actin cortex by treatment with DNase I and $\beta$-mercaptoethanol which led to a decrease in apparent spring constant of $44 \%$ and $65 \%$. ${ }^{[249]}$ The values detected in this work are slightly below this determined decrease. A possible origin of this discrepancy is the use of a minimal actin cortex instead of natural actin cortex harboring many actin binding proteins.

In addition, it should be mentioned that PSLBs show higher lateral membrane tensions than plasma membranes out of top-down experiments. Plasma membranes with an attached actin cortex show a lateral membrane tension of about $0.01-0.1 \mathrm{mN} \cdot \mathrm{m}^{-1} \cdot[249,250]$ Considering the different model systems, a rising tendency of higher lateral membrane tensions from plasma membranes via pure DOPC lipid bilayers up to positively charged lipid bilayers is observed.[116,233,249-251]. Therefore, positively charged lipids seem to have a stronger interaction with the self-assembled monolayer (SAM) on the gold surface.

The lateral membrane tension furthermore rises when the MAC is attached. It is reported in literature that binding of lipids reduces their lateral mobility, like it was shown for PtdIns [4,5] $\mathrm{P}_{2}$ in case of ezrin binding. ${ }^{[119]}$ Therefore, it is assumed that the attachment affects the positively charged lipids and the lateral mobility of these lipids is hindered. The ability of the lipids to follow upon indentation of PSLB is restricted as the lateral mobility is reduced due to the attached F-actin filaments. The anchored lipids may act as a diffusion barrier. The resulting higher mismatch of the chemical 
potentials between pore rim and pore center leads finally to a higher prestress resulting in a higher lateral membrane tension.

Furthermore, an observation of a large distribution of detected lateral membrane tensions was made. Among these membrane tensions some accumulations were found, suggesting that specific accumulations correspond to single GUV patches. Interestingly, these accumulations were found with and without attached F-actin network. Certainly, the detected lateral membrane tensions could be associated to accurate experimental days. This dependency strongly supports the made hypothesis that single experiments quantifying one specific F-actin morphology led to a specific apparent spring constant (cf. Figure 4.43). Due to the fact that no correlation between a special F-actin network morphology and the detected lateral membrane tension could be made this hypothesis cannot be verified. The findings are restricted to different lateral membrane tensions which originate from different GUV patches. One possible reason for this large heterogeneity of observed lateral membrane tensions with and without F-actin network could already originate from the lipid bilayer. A heterogeneous distribution of the positively charged lipid DOTAP would lead to different densities of anchor points, which could explain the different observed morphologies. Accordingly, the detected lateral membrane tensions should already show a heterogeneity which is the case in the presented experiments. A heterogeneous lipid distribution is very likely, as this phenomenon was already reported in literature. ${ }^{252]}$ Larsen et al. showed a three times greater variation in lipid composition than the experimental uncertainties for GUVs with a diameter of 3-12 $\mathrm{mm}$ produced by electroformation. Experimental uncertainties were determined by measuring the same vesicle multiple times with interim defocusing. ${ }^{[252]}$ The presence of more positively charged lipids in a local area would provide more pinning points for the F-actin network which would lead to denser F-actin cortices (cf. chapter 4.2.3). Besides the prevented motility of the positively charged lipids, the resulting denser F-actin network could also have an impact on the lateral membrane tension. As shown in chapter 4.3.1, the attachment of MACs to a lipid bilayer changes the mechanical properties of the F-actin network and that the pinning point density has a significant impact on these properties. Furthermore, Lorenz et al. already showed that a natural cortex has an impact on the lateral membrane tension of plasma membranes. ${ }^{[249]}$ Therefore, it seems reasonable that mechanical impact is contributed from the MAC to the lipid bilayer. In conclusion, it is assumed that the impact of F-actin networks on PSLBs is dependent on the amount of membrane-cortex linkages.

The heterogeneous distribution was already observed at PSLBs without attached MAC. In line with the assumption that positively charged lipids seem to have a stronger interaction with the SAM this could explain why the detected F-actin network morphologies show a preference to the pore rim. This preference was also observed in 
earlier studies. ${ }^{[16]}$ One possibility to verify this assumption is the use of a labeled DOTAP to qualitatively compare the amount of fluorophore in the PLSB with the one on the pore rim. Due to the mentioned fluorescence quenching of the gold layer the comparison would be difficult to implement. In literature there is another reported functionalization method using silicon monoxide which is less affected by quenching of the fluorescence signal. ${ }^{[253]}$ In conclusion, there are some strategies presented to tackle the observed problems. One necessary improvement is the correlation of single GUV patches before and after MAC attachment.

In case of PSLBs with a diameter of $5.0 \mu \mathrm{m}$ the heterogeneity in lateral membrane tensions of pure lipid bilayers is most obvious. Values relating to one experimental day (day 2, red) exceed almost completely the detected spring constant for PSLBs with attached MAC. Besides the already discussed heterogeneous distribution of the positively charged lipid, a difference of the spreading surface, the functionalized substrate, between different days can also be a reason for this finding. The observation of a narrower distribution on one experimental day supports this assumption. A study using ellipsometry, infrared spectroscopy and electrochemical measurements showed that short-chained SAMs are worse packed and more inhomogeneous than long-chained ones. ${ }^{[254]}$ In this work mercaptoethanol was used for assembling the SAM, carrying a short carbon-chain. Inhomogeneity within the SAM closely around the pores would lead to a varying adhesive force to the lipid bilayer. Therefore, the flow of the lipids inside the PSLB during indentation is altered and leads to a different apparent spring constant. This could also be an explanation for the observed heterogeneities among different experimental days and also different GUV patches. 


\subsection{Alteration of F-actin Network Morphology by Actin Binding Proteins}

In cellular actin networks the influence of single cross-linking proteins is hard to distinguish as the vast array of different actin binding proteins (ABP) lead to several effects which cannot be associated to a single ABP. Using the presented minimal bottom-up approach of MACs, the successive addition of single ABPs can be studied.

\subsubsection{Influence of Fascin}

- Experiments in this chapter have been published in "Influence of cross-linkers on ezrin-bound minimal actin cortices" by M. Schön, I. Mey and C. Steinem, submitted to Prog. Biophys. Mol. Biol. 2018, accepted ${ }^{[64]}-$

To investigate the influence of the actin binding protein fascin on MACs two approaches have been pursued. Fascin is known to induce bundling of filaments (lat. fascin: to bundle) as described in chapter 1.3. In a first approach fascin was introduced right before the polymerization was induced by adding F1 solution. Therefore, G-actin monomers and fascin were present during the polymerization process. These networks are hereafter referred to as pre-polymerized fascin treated networks. In a second approach F-actin networks were polymerized in the absence of fascin and subsequently attached to an ezrin decorated lipid bilayer doped with $4 \mathrm{~mol} \% \operatorname{PtdIns}[4,5] \mathrm{P}_{2}$ forming an MAC as reported in chapter 4.2. The actin binding protein fascin was then added onto these MACs to study its effect. These experiments are hereafter referred to as in situ (lat.: on site) experiments.

Pre-polymerized fascin treated networks were prepared by addition of $0.33 \mu \mathrm{M}$ and $0.63 \mu \mathrm{M}$ fascin resulting in a fascin:G-actin ratio of 1:25 and 1:13. These 3D networks (Figure 4.46) were analyzed in a microfluidic chamber (chapter 3.3.5) and further on attached to the lipid bilayer.

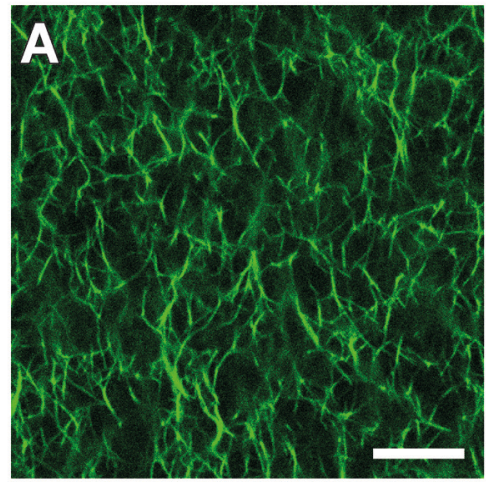

G-actin

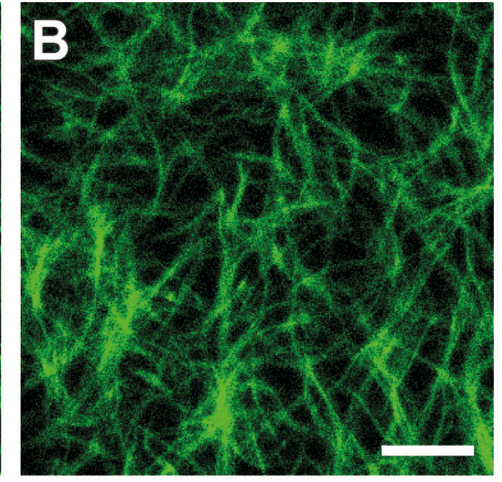

fascin:G-actin 1:25

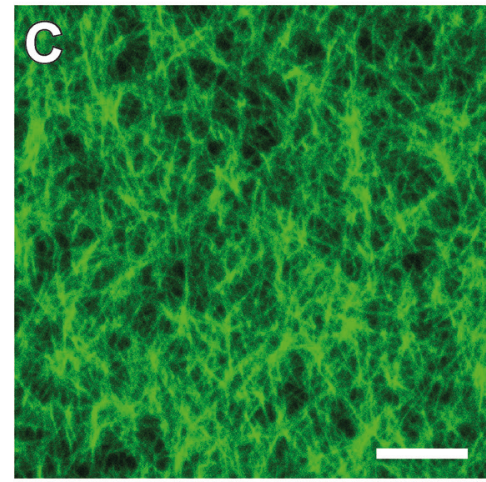

fascin:G-actin 1:13

Figure 4.46: Fluorescence Airyscan micrographs of 3D F-actin network gels in dependence of fascin concentration. ${ }^{[64]}$ A: F-actin network without fascin, labeled with 
Alexa Fluor ${ }^{\circledR}$ 488. B: Addition of $0.33 \mu \mathrm{M}$ fascin leads to bundled networks. C: Addition of $0.63 \mu \mathrm{M}$ fascin leads to even more bundled networks. Images are projections of $8 \mu \mathrm{m}$ high z-stacks. Scale bars: 15 um. Modified from Schön et al.[64]

In contrast to 3D F-actin without an actin cross-linker (Figure $4.46 \mathrm{~A}$ ), the presence of fascin induces strong bundling (Figure 4.46 B-C) which can be observed as the filaments show less curvature and apparently longer filament contour lengths. Besides these obvious effects, the longer filaments appear in a higher fluorescence intensity (Figure $4.46 \mathrm{C})$ which is assumed to be related to bundled filaments. The fact that fascin induces straight bundles was already reported by Honda et al. ${ }^{[255]}$ They showed that polymerization of G-actin in presence of fascin encapsulated in a liposome led to lemon shaped liposomes with straight rigid protrusions. It was shown that the rigid fascin induced actin bundles were able to deform these liposomes. Therefore assumed, bundling of F-actin to long and straight filaments occurs. An increasing fascin portion from 1:25 (Figure 4.46 B) to 1:13 (Figure 4.46 A) leads to a more pronounced effect.

After attachment of the cross-linked network to a lipid bilayer, a different morphology is observed. These MACs show less bundling compared with 3D F-actin gels. More precisely, pre-polymerized fascin treated networks with a ratio of 1:25 show a similar morphology (cf. Figure 4.47 A) to those obtained without fascin treatment.
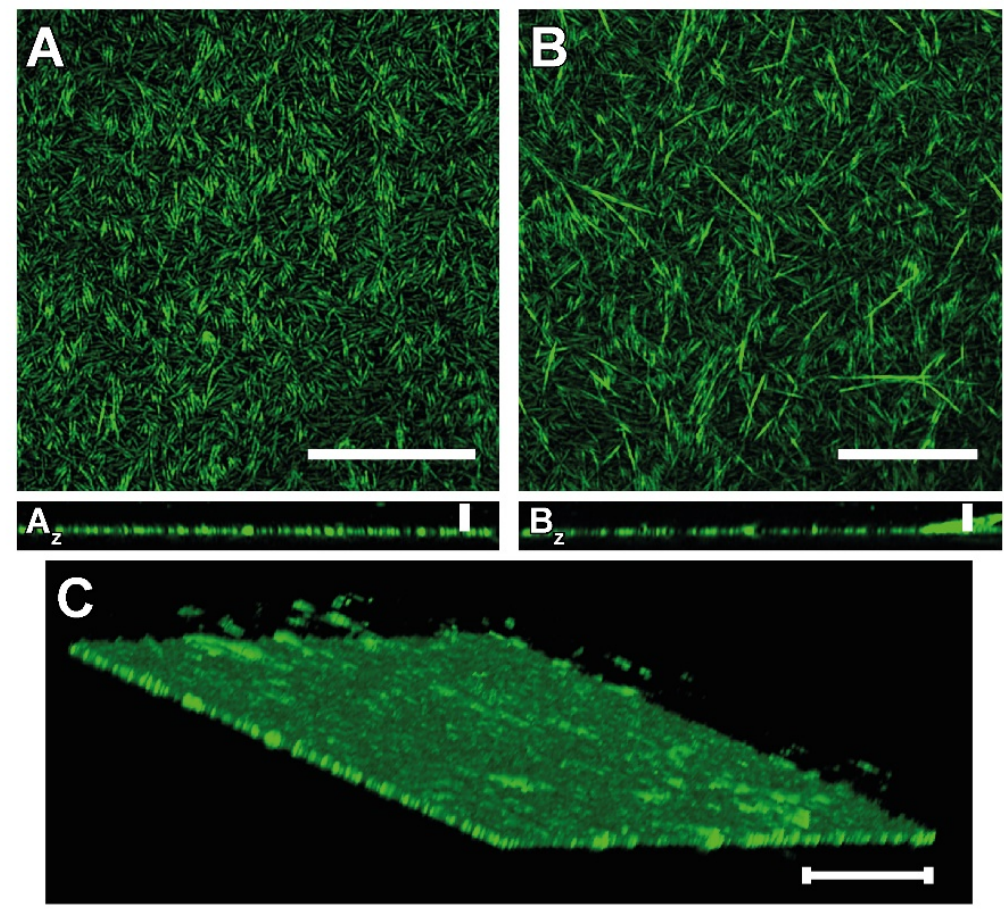

Figure 4.47: Confocal Airyscan micrographs of attached pre-polymerized fascin treated networks. ${ }^{[64]}$ Networks were labeled with Alexa Fluor ${ }^{\circledR} 488$ and attached via ezrin T567D to a lipid bilayer containing $4 \mathrm{~mol} \% \mathrm{PtdIns}[4,5] \mathrm{P}_{2}$. A-B: Morphology of an attached network with a fascin:G-actin ratio of 1:25 (A) or 1:13 (B). C: 3D view of pre-polymerized fascin treated F-actin network shown in $\mathbf{B}$ ). Scale bars: $15 \mu \mathrm{m} . \mathbf{A}_{\mathbf{z}}-\mathbf{B}_{\mathbf{z}}$ : Corresponding crosssectional fluorescence micrographs. Scale bars: $5 \mu \mathrm{m}$. Modified from Schön et al. ${ }^{[64]}$ 
Only by increasing the fascin:G-actin ratio up to 1:13 clear visible bundle like structures become visible in the fluorescence micrographs (Figure $4.47 \mathrm{~B}$ ). These structures appear to be longer than the filaments which were observed in experiments without an actin cross-linker as presented in chapter 4.2. The majority of the detected filaments remains relatively small and not clearly bundled to larger structures. Discussing the attachment process of MACs in absence of cross-linkers (cf. chapter 4.2.5) the hypothesis was made that a sorting-effect during the attachment process occurs, because preferably small filaments were bound to the ezrin decorated surface. Considering this observation, it seems reasonable that the pre-polymerized cross-linked and longer filaments were sorted out during attachment. Since the bundled, long filaments observed in the 3D F-actin gel could not be found in the attached F-actin network, the hypothesis of a sorting-effect is supported by these experiments using a pre-polymerized, cross-linked F-actin network. $z$-stacks of both networks show that in case of a fascin ratio of 1:25 (Figure $4.47 \mathrm{~A}_{\mathrm{z}}$ ) no difference to untreated networks (chapter 4.2, Figure 4.49) was observed, while in case of 1:13 the bundle like structures appear to lie on top of the MACs (Figure $4.47 \mathrm{~B}_{\mathrm{z}}$ ). Furthermore, a 3D view (generated with the software ZEN 2.3 SP1, Carl Zeiss, Oberkochen, Germany) shows higher fluorescence intensity in layers above the MAC (Figure $4.47 \mathrm{C}$ ).

To conclude, for low fascin concentrations the pre-polymerized F-actin filaments, cross-linked by fascin, are sparsely attached to the network, supporting the hypothesis of a sorting-effect preferring smaller filaments. In higher concentrations an alteration of the network morphology was observed. Thus, the procedure of attaching an already treated pre-polymerized F-actin network cannot fully reflect the activity of fascin on the F-actin network as most treated filaments do not result in the MAC.

In the second approach, fascin was added in situ after a successful formation of the MAC. As each sample was rinsed to remove unbound filaments, no exact fascin:G-actin ratio can be given using this preparation technique. Fascin was added in distinct concentrations of $36 \mathrm{nM}$ and $73 \mathrm{nM}$ directly on top of the MAC. The amount of fascin remains identical to experiments using pre-polymerized fascin treated networks, but the volume of the measuring chamber is larger. Thus, these concentrations would also lead to fascin ratios of 1:25 (36 $\mathrm{nM})$ and 1:13 (73 $\mathrm{nM})$, if all G-actin monomers were accessible. 

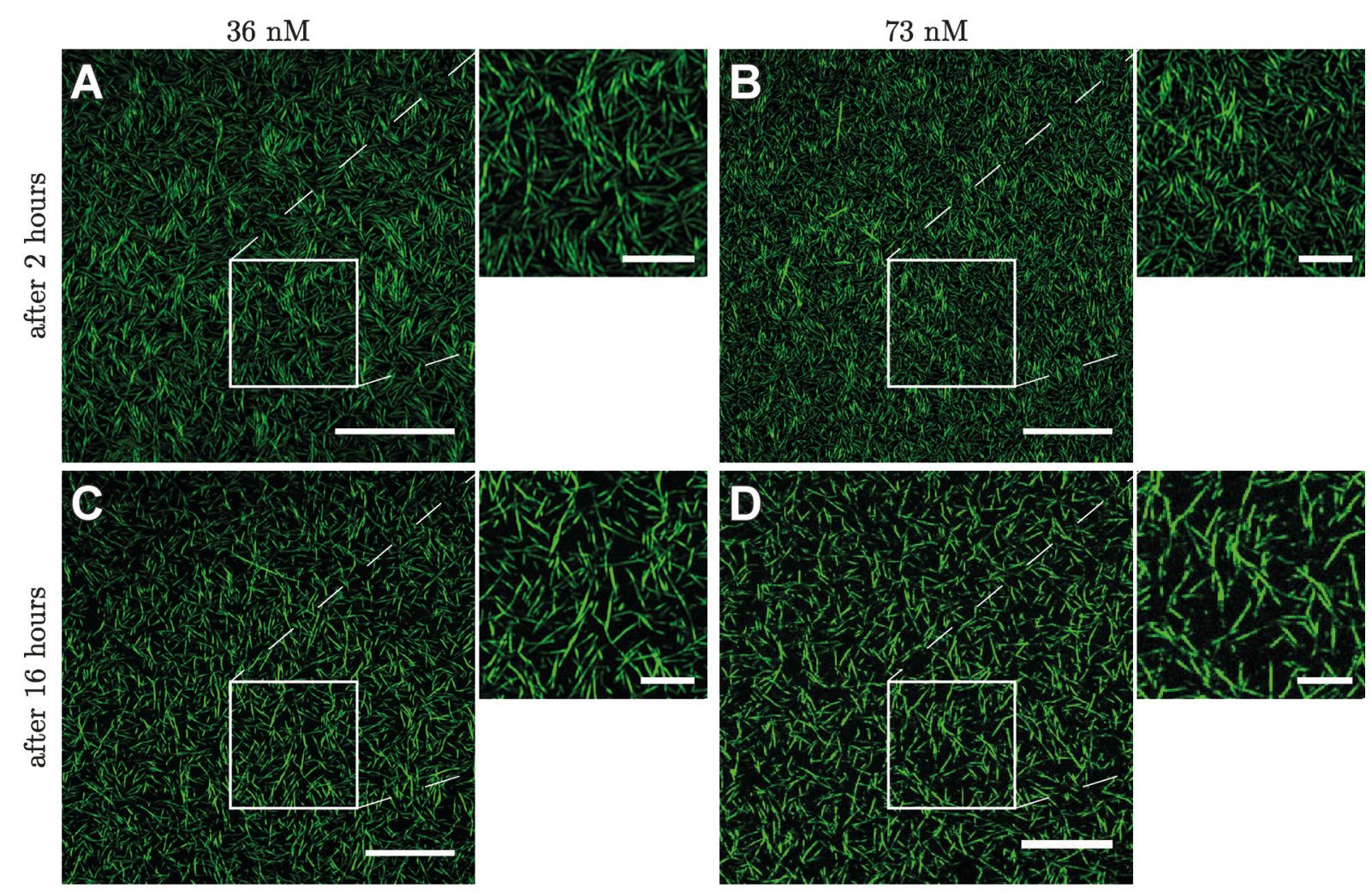

Figure 4.48: Influence of fascin addition to membrane attached F-actin networks. ${ }^{64]}$ Fluorescence Airyscan micrographs of MACs treated with $36 \mathrm{nM}(\mathbf{A} / \mathbf{C})$ or $73 \mathrm{nM}(\mathbf{B} / \mathbf{D})$ fascin. Images were collected after 2 hours (A-B) and 16 hours (C-D). Magnifications are shown for clarification. Scale bars: $15 \mu \mathrm{m}$, magnifications: $5 \mu \mathrm{m}$. Modified from Schön et al.[64]

Two hours after fascin addition, first bundle like structures appear in the fluorescence micrographs of the F-actin network's morphology, as shown in Figure $4.48 \mathrm{~A}+\mathrm{B}$ for both fascin concentrations. Specimens were checked again after 16 hours: a long-time rearrangement of the F-actin network occurred, resulting in less filaments attached to the lipid bilayer compared to 14 hours before, which appear to be longer and more bundled as the filaments show less curvature. After image processing and skeletonization via artificial retina (chapter 3.6.1.1), the filament length was evaluated from log-normal distributions. These log-normal distributions were evaluated as described in detail before (cf. chapter 4.2.1). While untreated MACs have a length of $(3.2 \pm 3.5) \mu \mathrm{m}$, the length of treated MACs increases up to $(3.9 \pm 3.2) \mu \mathrm{m}(36 \mathrm{nM})$ and $(3.9 \pm 4.1) \mu \mathrm{m}(73 \mathrm{nM})$ after 2 hours. The effect of increasing filament length is even more pronounced after 16 hours. MACs treated with $36 \mathrm{nM}$ fascin show a filament length of $(5.0 \pm 3.4) \mu \mathrm{m}$, while with a higher concentration of $73 \mathrm{nM}$ the filament length increases up to $(7.4 \pm 4.4) \mu \mathrm{m}$ (cf. Table 4.8). 
Table 4.8: Alteration of filament lengths after addition of $36 \mathrm{nM}$ or $73 \mathrm{nM}$ fascin.

\begin{tabular}{c|c|c}
$\begin{array}{c}\text { filament length } / \mathbf{~} \mathbf{m} \\
\left(\boldsymbol{\mu}^{*} \pm \boldsymbol{\sigma}^{*}\right)\end{array}$ & $\mathbf{3 6} \mathbf{~} \mathbf{M}$ fascin & $\mathbf{7 3} \mathbf{~} \mathbf{M}$ fascin \\
\hline $\begin{array}{c}\text { Before addition } \\
\text { (2D F-actin cortex) }\end{array}$ & $3.2 \pm 3.5$ & $3.2 \pm 3.5$ \\
$\mathbf{2}$ hours after addition & $3.9 \pm 3.2$ & $3.9 \pm 4.1$ \\
$\mathbf{1 6}$ hours after addition & $5.0 \pm 3.4$ & $7.4 \pm 4.4$
\end{tabular}

Evaluation of the node densities via artificial retina was conducted in order to obtain a quantitative parameter for the network density as presented in chapter 4.2 (cf. Figure 4.49). Pre-polymerized fascin treated (fascin:G-actin ratio of 1:25) networks showed a node density of $(1.3 \pm 0.2) \mu^{-2}$ (mean \pm standard deviation), which is almost identical to the node density evaluated from pre-polymerized F-actin networks without fascin treatment. For networks with a ratio of 1:13 a larger node density of $(1.7 \pm 0.3) \mathrm{\mu m}^{-2}$ was observed (cf. Figure 4.49). In total, this demonstrates, that the prominently observable bundling effect in $3 \mathrm{D}$ network gels could not be found when these networks were attached to a lipid bilayer. Then, the bundling effect is only present at high concentrations of fascin and barely to see at low concentrations. In case of in situ experiments, the node densities of the various concentrations at two times were determined. In case of $36 \mathrm{nM}$ fascin addition, the node density decreases from $(1.1 \pm 0.1){\mu \mathrm{m}^{-2}}^{2}$ after 2 hours to $(0.7 \pm 0.1){\mu \mathrm{m}^{-2}}^{2}$ after 16 hours. The decrease in node density is more pronounced upon addition of $73 \mathrm{nM}$ fascin: it decreases to $(0.89 \pm 0.02) \mu^{-2}$ after 2 hours and to $(0.3 \pm 0.2) \mu^{-2}$ after 16 hours (cf. Figure 4.49). Noteworthy, the node density of untreated MACs remains the same for 16 hours. 


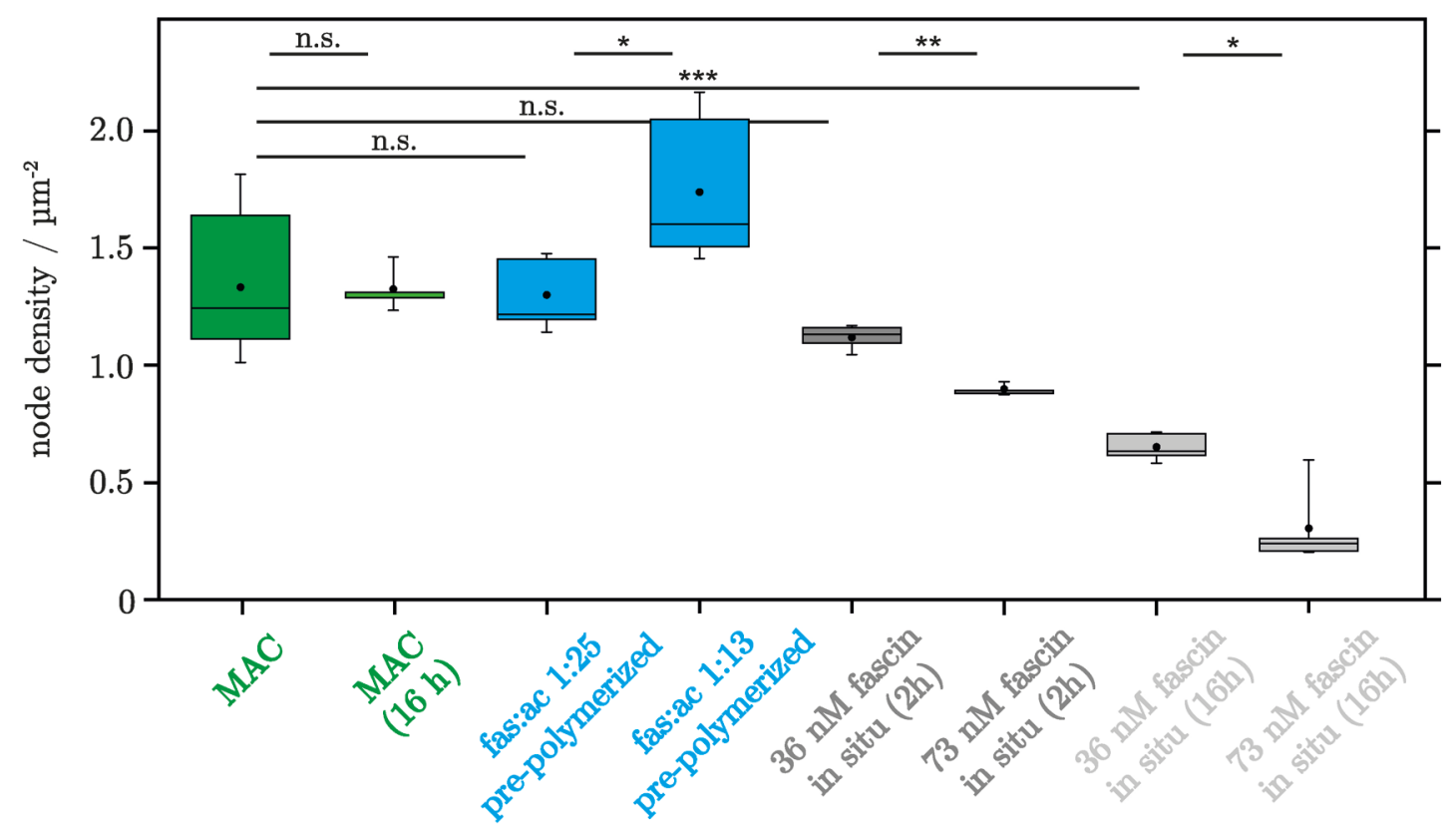

Figure 4.49: Node densities of various F-actin networks showing the influence of fascin. ${ }^{[64]}$ The node density of a pure F-actin network bound to an ezrin decorated lipid bilayer doped with $4 \mathrm{~mol} \% \mathrm{PtdIns}[4,5] \mathrm{P}_{2}$ remains the same from directly after attachment (MAC) to 16 hours (MAC, 16h). Pre-polymerized fascin treated networks show a similar node density of about $1.3 \mathrm{\mu m}^{-2}$ in case of a fascin:G-actin ratio of 1:25 (fas:ac 1:25) or an even higher node

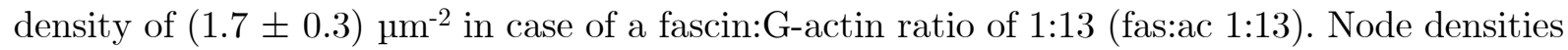
of in situ experiments show a concentration and time dependent decrease. Addition of $36 \mathrm{nM}$ fascin decreases the node density to $(1.1 \pm 0.1){\mu m^{-2}}$ after 2 hours $(36 \mathrm{nM}, 2 \mathrm{~h})$ and further on to $(0.7 \pm 0.1) \mathrm{\mu m}^{-2}(36 \mathrm{nM}, 16 \mathrm{~h})$. Adding a higher concentration of $73 \mathrm{nM}$ amplifies the effect and decreases the node density to $(0.89 \pm 0.02){\mu \mathrm{m}^{-2}}^{2}$ after 2 hours $(73 \mathrm{nM}, 2 \mathrm{~h})$ and to $(0.3 \pm 0.2) \mathrm{\mu m}^{-2}$ (73 nM, 16h). Mann-Whitney $U$ tests were performed to show that two populations are not populations with equal means. Significance levels are: $5 \%(*), 1 \%(* *)$ and $0.1 \%(* * *)$ while n.s. displays two non-significant data sets. Box plots extent from $25^{\text {th }}$ to $75^{\text {th }}$ percentile and whiskers extent from $5^{\text {th }}$ to the $95^{\text {th }}$. Modified from Schön et al. ${ }^{[64]}$

To gather more information about the time resolved F-actin rearrangement, a time series of fluorescence micrographs after the addition of $73 \mathrm{nM}$ fascin was recorded (Figure 4.50). Interestingly, confocal micrographs of the flat MAC layer in $x-, y$-direction show only slight changes in the morphology of the MAC. Only by recording z-stacks, the total rearrangement of the morphologic changes becomes visible. As already indicated by the 3D view of Figure $4.47 \mathrm{C}$, bundle like structures appear on top of the flat MAC layer, so more than one z-plane had to be recorded. Therefore, subsequently $z$-stacks of a total height of $12 \mu \mathrm{m}$ were recorded and displayed as a projection to a $2 \mathrm{D}$ image for clear representation (Figure 4.50). These z-stacks reveal that bundled structures protrude in z-direction. 

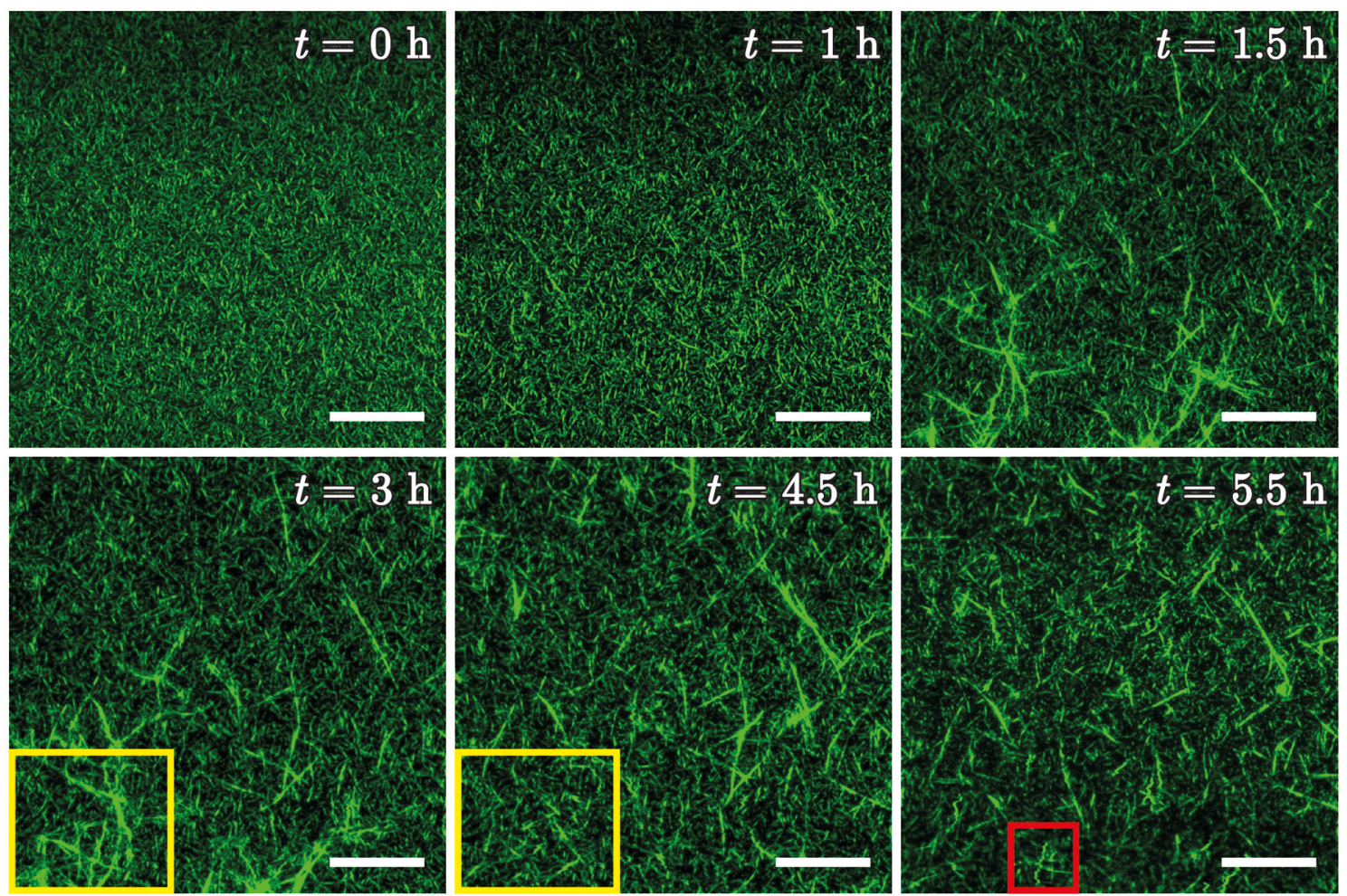

Figure 4.50: Time series of fluorescence Airyscan micrographs showing the influence of fascin. ${ }^{[64]}$ All images are projections with averaged intensity of $12 \mu \mathrm{m}$ high $z$-stacks into one layer. At the starting point $(t=0 \mathrm{~h})$ an untreated F-actin network is observed. One hour after addition of $73 \mathrm{nM}$ fascin first bundle like structures can be observed. Over time more and more bundle protrusions appear and vanish (yellow box). Bundles are highly mobile, so they appear to flicker inside the z-stack (red box). Scale bars: 15 um. Modified from Schön et al.[64]

In the observed area a few smaller protrusions appear already after one hour (Figure $4.50 t=1 \mathrm{~h}$ ). In the following 30 minutes, more bundled structures emerge at the bottom of the fluorescence micrograph (Figure 4.50, $t=1.5 \mathrm{~h}$ ). Larger bundles can detach from the surface, because they disappear in the interim of two images as it is shown in the yellow box. (Figure 4.50 compare $t=3 \mathrm{~h}$ and $t=4.5 \mathrm{~h}$ ). The protrusion of bundle structures is only found in z-direction, where they are loosely bound to the surface, because only one end can bind the ezrin decorated lipid bilayer. This can be observed as the movement of the filaments over the z-stack's measuring duration is flickering in the image (red box in Figure 4.50, $t=5.5 \mathrm{~h}$ ). 


\subsubsection{Influence of $\alpha$-Actinin}

- Experiments in this chapter have been published in "Influence of cross-linkers on ezrin-bound minimal actin cortices" by M. Schön, I. Mey and C. Steinem, submitted to Prog. Biophys. Mol. Biol. 2018, accepted ${ }^{[64]-}$

After characterization of fascin, the impact of $\alpha$-actinin to attached F-actin networks, by observation of morphology changes, was analyzed. In comparison to fascin, $\alpha$-actinin is not only known to be able to bundle F-actin in higher concentrations, it can also induce branching at low ratios of $\alpha$-actinin:G-actin as already reported in chapter 1.3. ${ }^{[256]}$ To verify this behavior, G-actin was polymerized in different ratios in presence of $\alpha$-actinin and analyzed in a microfluidic chamber.

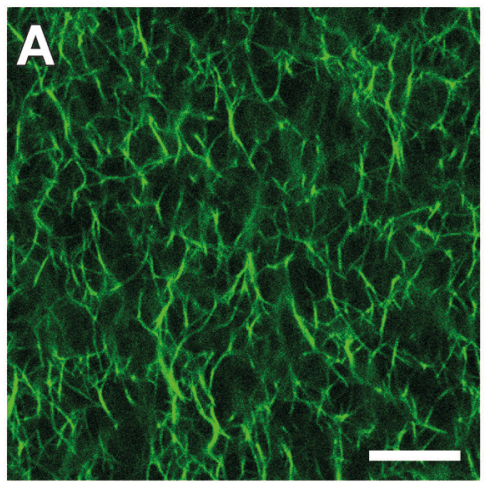

G-actin

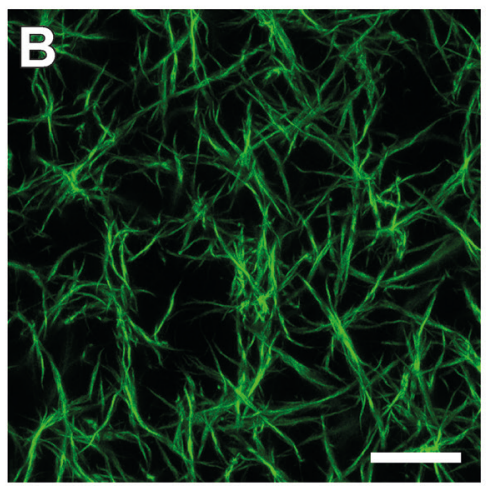

$\alpha$-actinin:G-actin 1:40

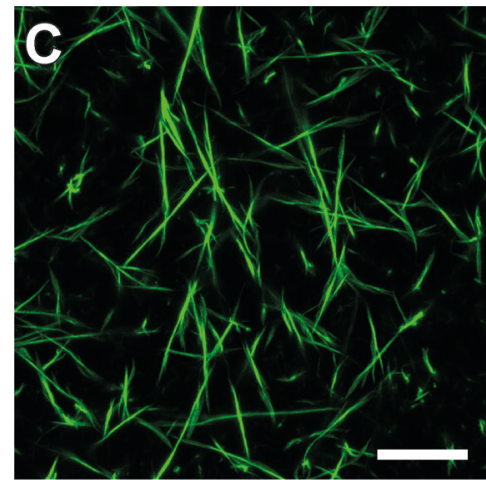

$\alpha$-actinin:G-actin 1:15

Figure 4.51: Fluorescence Airyscan micrographs of 3D F-actin networks gels polymerized in absence or presence of $\alpha$-actinin. ${ }^{[64]}$ In contrast to untreated $\mathrm{F}$-actin networks, which appear to be highly entangled (A), the obtained morphology of F-actin network gels polymerized in presence of $\alpha$-actinin differs greatly $(\mathbf{B}-\mathbf{C})$. B: Adding $0.21 \mu \mathrm{M}$ $\alpha$-actinin to an G-actin solution ( $\alpha$-actinin:G-actin ratio of 1:40) leads to polymerization of branched networks which appear as straight filaments with a high number of branching T-junction points. C: Adding a higher concentration of $0.55 \mu \mathrm{M} \alpha$-actinin ( $\alpha$-actinin:G-actin ratio of 1:15) leads to a formation of thick and straight bundles. All images are projections of $8 \mu \mathrm{m}$ high $z$-stacks. Scale bars: A: $15 \mu \mathrm{m}$ B-C: $20 \mu \mathrm{m}$. Modified from Schön et al. ${ }^{[64]}$

The obtained fluorescence micrographs (cf. Figure 4.51) show that the previously reported effect of $\alpha$-actinin on 3D F-actin gels was also found in 3D F-actin gels within this work. While a ratio of 1:40 (G-actin: $\alpha$-actinin) leads to branched networks of straightened filaments, a higher ratio of 1:15 (G-actin: $\alpha$-actinin) showed only thick and almost linear filaments.

As a result of the gathered insights of F-actin attachment (cf. chapter 4.2.2) and the attachment of fascin treated F-actin gels (cf. chapter 4.4.1) to ezrin decorated lipid bilayers, which showed a preferable attachment of small filaments to the lipid bilayer, only in situ experiments were pursued for $\alpha$-actinin. $\alpha$-actinin was added in situ in 
concentrations of $12 \mathrm{nM}, 23 \mathrm{nM}$ and $61 \mathrm{nM}$ (cf. Figure 4.52). Already low concentrations of $12 \mathrm{nM} \alpha$-actinin lead to bundling of actin filaments on a time scale of 3 hours (Figure 4.52 A) and further increase in bundling is observed over time (Figure $4.52 \mathrm{D})$. This morphologic change is also concentration dependent. An $\alpha$-actinin concentration of $23 \mathrm{nM}$ leads to more bundled structures (Figure $4.52 \mathrm{~B}$ ), up to whole spider-web like structures, already after 3 hours of incubation time (61 nM, Figure 4.52 C). An increase of bundling over time is observed for every concentration, leading to cross-linked and bundled clew structures, which show a high fluorescence intensity (Figure $4.52 \mathrm{~F}$ ). Similar to experiments with fascin, the cross-linked and bundled structures grow into the $z$-direction which is shown in $z$-stack images (Figure 4.52 $\mathrm{A}_{\mathrm{z}}-\mathrm{F}_{\mathrm{z}}$ ). The height of the formed bundled or spider-web like structures range from 8 to $10 \mu \mathrm{m}$ independent of the used concentration (Figure $4.52 \mathrm{~A}_{\mathrm{z}}-\mathrm{F}_{\mathrm{z}}$ ). Because the lipid bilayer remained unaffected from changes, a presentation was renounced for clarity.
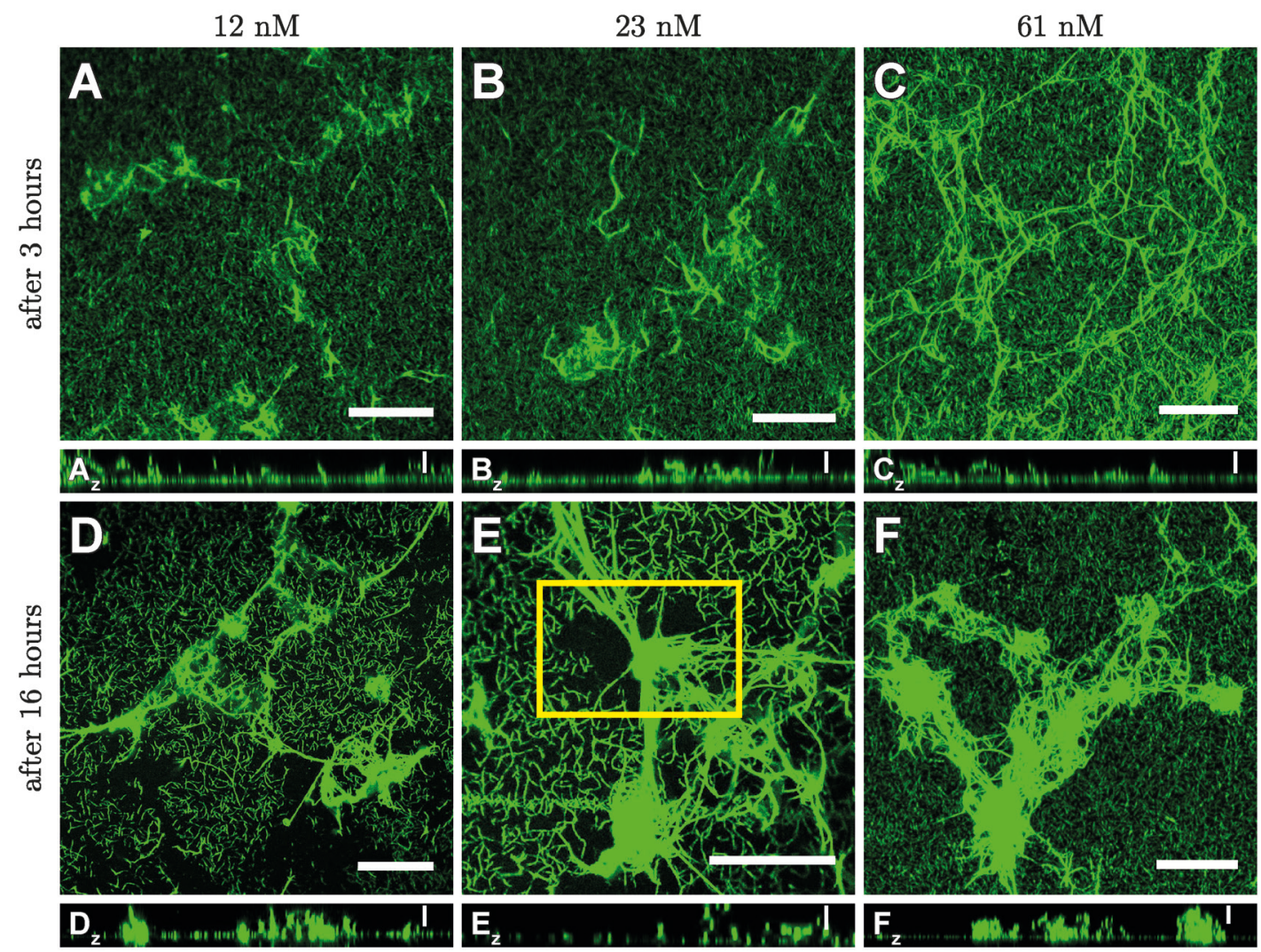

Figure 4.52: Influence of $\alpha$-actinin to minimal actin cortices in dependence of concentration and time. ${ }^{[64]}$ All images are $2 \mathrm{D}$ projections with averaged intensity of z-stacks. Addition of (A) $12 \mathrm{nM},(\mathbf{B}) 23 \mathrm{nM}$ or (C) $61 \mathrm{nM} \alpha$-actinin induces bundle formation which protrude into the $z$-direction $\left(\mathbf{A}_{\mathbf{z}}-\mathbf{C}_{\mathbf{z}}\right)$. At higher concentrations, spider-web like structures can be observed (C). Rearrangement occurs further on as tight bundle clew structures appears after 16 hours (D-F). All observed bundled structures show an extent of fluorescence intensity in z-direction of 8-10 $\mu \mathrm{m}\left(\mathbf{A}_{\mathbf{z}}-\mathbf{F}_{\mathbf{z}}\right)$. Scale bars: A-F: $15 \mu \mathrm{m} . \mathrm{A}_{z}-\mathrm{F}_{\mathbf{z}}: 5 \mu \mathrm{m}$. Modified from Schön et al. ${ }^{[64]}$ 
Additionally, fluorescence images strongly suggest a recruitment of F-actin from the membrane bound MAC, as areas close to bundle junction points (Figure 4.52, yellow box) appear to be decreased in fluorescence intensity. In contrast to fascin treated MACs, the determined node densities showed no comparable tendency between different specimens, since the very large rearrangements of the MAC alters the node density already on one single silicon substrate in a large range. Node densities of $(0.6 \pm 0.2) \mathrm{\mu m}^{-2} \quad(12 \mathrm{nM} / 23 \mathrm{nM})$ or $(0.8 \pm 0.2) \mathrm{\mu m}^{-2}(61 \mathrm{nM})$ were determined, displaying the mean value for all preparations at a given $\alpha$-actinin concentration. Interestingly, detected node densities of one single sample were ranging from

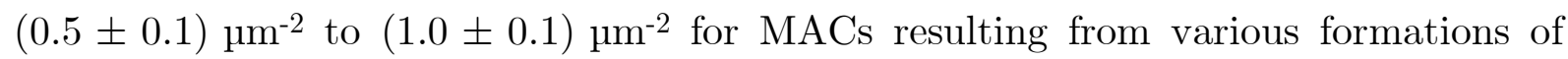
bundled structures. A tendency to smaller node densities close to junction points (as shown in Figure 4.52, yellow box) was observed. Furthermore, additional strong rinsing was not able to remove formed bundles from the attached MAC. The formed bundles clearly moved but were not striped away (Figure 4.53).
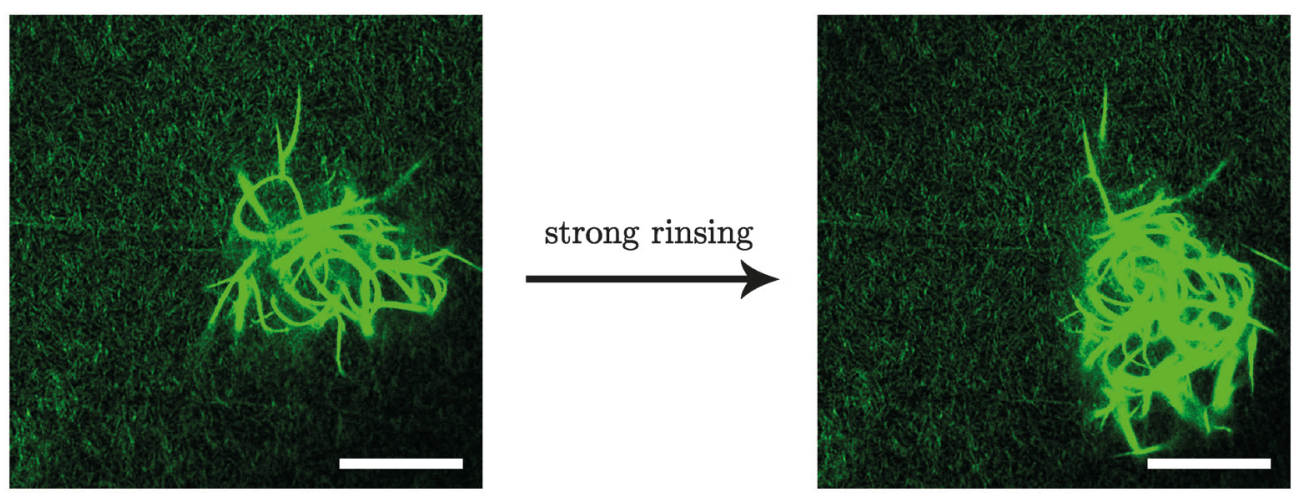

Figure 4.53: Strong rinsing leads to bundle displacement. A clew structure of F-actin: $\alpha$ actinin bundles labeled with Alexa Fluor ${ }^{\circledR} 488$ was tried to remove with strong rinsing by an Eppendorf pipette. The bundle was formed by adding $61 \mathrm{nM} \alpha$-actinin to a preformed MAC, 16 hours before. Scale bars: $15 \mu \mathrm{m}$.

While observing these different bundled structures on top of the nearly flat F-actin layer attached to the lipid bilayer, it remains unclear how these structures form. For that, a time series was recorded, tracking the influence of $61 \mathrm{nM} \alpha$-actinin. The corresponding fluorescence micrographs reveal a continuous growing of bundle like structures evolving from junction points on top of the MAC. 

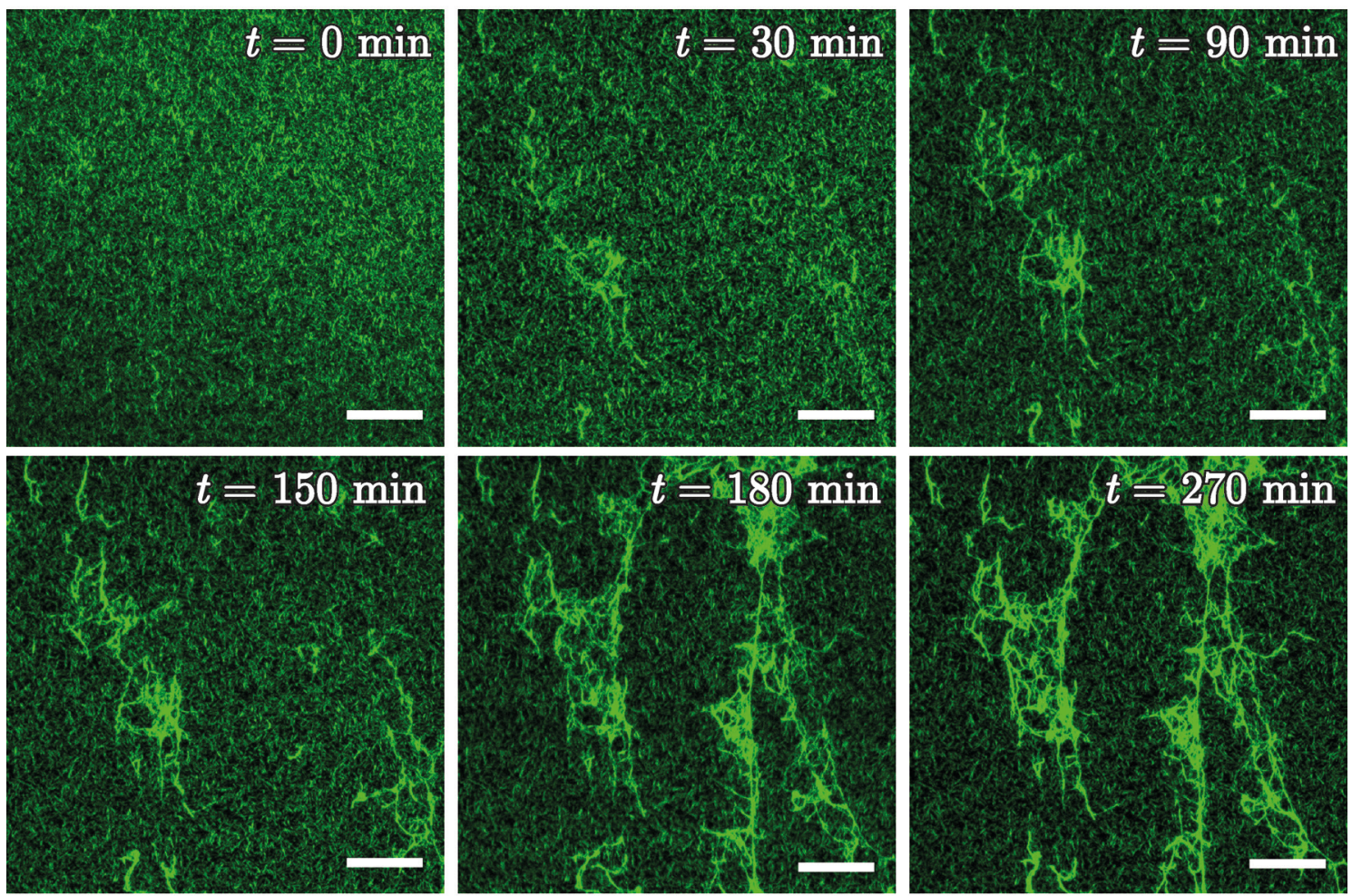

Figure 4.54: Time series of fluorescence Airyscan micrographs showing the influence of $\boldsymbol{\alpha}$-actinin. ${ }^{[64]}$ All images are 2D projections with averaged intensity of $10 \mu \mathrm{m}$ high $z$-stacks. At the starting point $(t=0 \mathrm{~h})$ an untreated F-actin network labeled with Alexa Fluor ${ }^{\circledR} 488$ is observed. Already 30 minutes after addition of $61 \mathrm{nM} \alpha$-actinin first larger aggregates of F-actin are detectable. In the following two hours bundled structures grow close to these first observed aggregates. After 3 hours a spider web like structure is observable which does not change significantly up to 4.5 hours. Scale bars: 15 m. Modified from Schön et al. ${ }^{[64]}$

First, a few aggregates and bundles appear, which can be observed as brighter structures (Figure 4.54, $t=30 \mathrm{~min}$ ). These structures proceed to growth in size originating at the positions of first bundled structures (Figure 4.54, $t=90 \mathrm{~min}$ and $t=150 \mathrm{~min}$ ). These first positions seem to be the already mentioned junction points to the lower layer of F-actin. While these structures grow, connections between junction points are established (Figure 4.54, $t=180 \mathrm{~min}$ ), until whole spider-web like structures are observed. Further on, only little changes in these spider web-like structures appear and the formed structures are highly stable as they cannot be rinsed away as shown in Figure 4.53. The underlying lipid bilayer remained unaffected again, which is not shown for clarity. 


\subsubsection{Discussion of the Influence of Actin Binding Proteins to MACs}

$\alpha$-actinin and fascin influence MACs in different ways, but they have one feature in common: both can rearrange a physiologically bound F-actin network and induce modifications of the thin MAC into the third dimension. The presented experiments, considering a pre-polymerized cross-linker treated 3D F-actin gel, support the findings of chapter 4.2.5, which showed that a sorting effect during the attachment to an artificial lipid bilayer occurs. It was shown that long actin filaments, observed in 3D gels, could not be attached to ezrin decorated lipid bilayers doped with different PtdIns[4.5] $\mathrm{P}_{2}$ ratios. The observation of only small filaments supports the hypothesis that longer filaments are either sorted out or broken during attachment process. Longer or bundled cross-linked filamentous structures were therefore broken or sorted out. Thus, longer cross-linked filaments are only barely to see, which is consequent with the described sorting effect. Consequently, in situ experiments with actin binding proteins are more advantageous than using pre-polymerized bundled filamentous structures. The experiments using pre-polymerized F-actin networks in $3 \mathrm{D}$ were important as preliminary results, since they verified the reaction conditions by showing that bundling and branching occurs in agreement with results reported in literature. ${ }^{[255-259]}$ In case of pre-polymerized $\alpha$-actinin treated network gels strong bundling at ratios above 1:20 (actinin:G-actin) and branching at lower ratios were demonstrated (cf. Figure 4.51) as described before.[256]

In a natural cell cortex, "active" actin binding proteins like myosin motor proteins as well as "passive" actin binding proteins or cross-linking proteins are present. In case of myosin motor proteins it is known that force is generated via "walking" of myosin between antiparallel actin filaments, whereas the observed dynamics in this work are induced by binding of the cross-linkers. ${ }^{[82,260]}$ In case of fascin, the interaction with actin was found to be reversible with a half-life of 6-9 seconds which enables a dynamic cross-linking effect.[261] These dynamics can be an enhancing factor of the observed rearrangement process, which comes along with bundle formation. Another origin of the surprisingly dynamic process is that the F-actin network is attached via the protein ezrin, which provides a binding kinetic instead of a fix bond. An interplay of both binding processes is probably leading to a rearrangement as observed in the experiments. Bundle formation itself was expected, as fascin is known to bundle actin filaments (cf. chapter 1.3) and it is also directly linked to reports of filopodia formation, which are long membrane protrusions pushed by actin bundles. ${ }^{[34,262]}$ Depletion experiments by RNA interference in mammalian cells revealed a loss of filopodia structures indicating that fascin is necessary to form filopodia. [32]

Furthermore, the bundling formation was found to be more effective during the polymerization process instead of the bundling of preformed filaments. ${ }^{[263]}$ Kureishy et 
al. reported that various factors influence the fascin induced bundling formation. It is described that already loosely linked filaments can also enhance the bundling effect. It is assumed, that quite densely attached MACs used in this work, seem to provide enough already (loosely) packed filaments, which then can easily be bundled. As a result, efficient bundling occurs, which can be observed within two hours after fascin addition (cf. Figure 4.50). The increase of bundling as a result of higher fascin concentrations seems reasonable, considering these findings reported in literature. A repetition of the presented experiments utilizing a denser MAC by using 6-8 mol \% PtdIns $[4,5] \mathrm{P}_{2}$ instead of $4 \mathrm{~mol} \%$ and subsequent comparison of observed bundling dynamics could verify the assumption that the dynamic is dependent on the available loosely packed actin filaments.

Regarding the protrusion's orientation of the obtained bundles, it is reported in literature that the fast-growing barbed ends of F-actin are orientated towards the plasma membrane. ${ }^{[34,264,265]}$ In cells it was observed that elongation of bundled structures within filopodia promotes cell migration or extension. Considering the experiments of this work, only a hypothesis can be proposed. It might be possible that the barbed ends of the filaments in our setup orientate towards the artificial bilayer and therefore the pointed ends protrude into the z-direction because of the bundle stiffness. As the artificial minimal model used in this work consists of only three proteins, it seems very unlikely that bundled structures show a specific orientation. Actin filaments are stabilized with phalloidin and the polymerization treadmilling is hampered. Therefore, both filament's ends do not differ in their dynamics. Nevertheless, a forming of filopodia-like lipid protrusions in our setup is not possible due to the underlying solid support. The use of pore spanning lipid bilayers could be an advantage in studying the direction of protrusion formation and could give a hint if there is a direction of filament's protrusion, even with stabilized filaments. In total, it seems most likely that the dynamics of filament movement are induced by fascin binding rate, the un-/binding rate of ezrin and thermal fluctuations during the CLSM measurement. As ezrin is only present in the plane of the membrane, rebinding might be hampered as the stiff bundle can detach from the membrane plane and therefore detachment happens due to lost contact to ezrin binding sites. The detachment could be supported by the fact that fascin bundles are stiffer compared to entirely actin fibers. Mansson et al. found a general dependency that fascin bundling of actin filaments results in a 15-fold increase in persistence length, therefore reducing the flexibility of the filaments. $[33,266]$

The obtained maximum bundle length of $(7.4 \pm 4.4) \mu \mathrm{m}$ (16 hours after addition of $73 \mathrm{nM}$ fascin) is comparable to some observations reported in literature. It was reported that fascin protruded nerve growth-cone filopodia show lengths up to $10 \mu \mathrm{m}$. Depending on the system, also larger filopodia lengths up to $40 \mu \mathrm{m}$ could be found in sea-urchin 
embryos. ${ }^{[267]}$ In this work the resulting, final contour length was hard to determine, as most filaments lost the contact to the lipid bilayer (cf. Figure 4.50, yellow box) and were not accessible for further evaluation. The observed maximal 2.5-fold extension from 3.2 to $7.4 \mathrm{\mu m}$ indicates that the dynamic process of bundle formation maybe was not finished. Furthermore, it was shown that during MAC formation a sorting or breaking effect of filaments occurs, which generally leads to small filaments on the ezrin decorated lipid bilayer. Initially shorter filaments would also explain a growth to a maximum length of $(7.4 \pm 4.4) \mu \mathrm{m}$ and not reaching length scales above $10 \mu \mathrm{m}$ or more. As most bundled structures loose contact to the lipid bilayer this issue is restricted by the experimental setup.

In contrast to fascin which is widely, specifically targeted to filopodia, $\alpha$-actinin is found in antiparallel actin-bundled structures at many different sites of the cell, such as z-disks of skeletal muscles, leading edges of motile cells or stress fibers. ${ }^{[33]}$ The main difference between both cross-linkers is that $\alpha$-actinin is a homodimer instead of a single protein and more importantly that the head-to-head distance is $20-30 \mathrm{~nm}$ instead of $5-8 \mathrm{~nm}$ in case of fascin. ${ }^{[33,263]}$ Due to the larger distance between two actin fibers and therefore the accessibility for myosin motor proteins in the bundles, most dynamic processes presented in literature contain motor proteins and it is hard to distinguish how $\alpha$-actinin contributes. In this work the minimal system was used to show that $\alpha$-actinin induces dynamic processes in an MAC. Recruitment of actin filaments out of the MAC 2D layer was observed resulting in cross-linked spider web-like structures lying on top of the 2D layer. This assumption is supported by a decrease in the node density of the underlying actin layer from 1.4 nodes per $\mu^{2}$ to $0.6-0.8$ nodes per $\mu^{2}$. Furthermore, it was shown that the node density decreases from $1.0 \mathrm{\mu m}^{-2}$ to $0.5 \mathrm{\mu m}^{-2}$ when getting closer to the observed junction points. These junction points connect the spider-web like structures and flat MAC layer beneath. They are believed to provide an interconnection of the spider web-like structures and the 2D actin layer underneath. It can be assumed that the growth of the structures stops when too little F-actin material is close enough to the junction points. This assumption is based on the fact that the proximity of loosely bound filaments has a strong impact on the cross-linking effect. ${ }^{[263]}$ As shown for fascin bundling, dynamics are enhanced when more loosely packed actin filaments are present. In contrast to fascin, the bundled structures of $\alpha$-actinin are less rigid and the distance between two filaments is 4 - to 6 -fold larger. The lower rigidity of the bundles could be a reason why the $\alpha$-actinin bundles are not detaching from the MAC like fascin induced bundles do. Also, the unbinding rate of $\alpha$-actinin to actin is slower than for fascin-actin, as the half-life of the actinin-actin bond was determined to be $(29 \pm 13)$ seconds. ${ }^{268]}$

In conclusion, dynamic processes of membrane coupled MACs induced by the influence of cross-linking proteins could be shown. Alterations and recruitment of attached 
F-actin network for bundle formation were presented by detecting changes of the network morphology. It is assumed that the dynamic membrane linkage enhances the induced rearrangement by fascin or $\alpha$-actinin. This hypothesis could in future be reviewed by comparison with a stronger membrane-actin cortex linkage like neutravidin or streptavidin (cf. chapter 4.3.1.1) does. More rigid fibers like fascin bundled actin filaments show a detachment from the membrane bound cortex, while filament bundles induced by $\alpha$-actinin show intact junctions to the MAC as strong rinsing could not tear the bundled structures away. Over all it was shown that the usage of MACs is a useful tool to investigate effects of single actin cross-linking agents. 


\section{Summary}

The main objective of this thesis was to create a minimal actin cortex (MAC) attached to a membrane, in a bottom-up approach to investigate characteristic features of the composite, like the filamentous actin (F-actin) organization, its network density and the mechanical properties of the F-actin networks. The construction of the MAC was based on a lipid bilayer doped with the receptor lipid L- $\alpha$-phosphatidylinositol4,5-bisphosphate (PtdIns $\left.[4,5] \mathrm{P}_{2}\right)$, an active mutant of ezrin and a pre-polymerized F-actin network. The use of the physiological linker protein ezrin, a member of the ERM protein family, which is known to connect the actin cortex with the plasma membrane in vivo, is a unique feature of this system compared to other setups reported in literature.

The amount of PtdIns $[4,5] \mathrm{P}_{2}$ within the artificial, solid supported lipid bilayer was varied to obtain different surface coverages of ezrin and consequently change the density of the provided connection points for the F-actin network. Ezrin surface coverage as a function of $\operatorname{PtdIns}[4,5] \mathrm{P}_{2}$ concentration was monitored by reflectometric interference spectroscopy (RIfS). A saturation of ezrin surface coverage was found at $>6 \mathrm{~mol} \mathrm{\%}$ PtdIns $[4,5] \mathrm{P}_{2}$.

By means of confocal laser scanning microscopy (CLSM) the organization of F-actin networks as a function of the $\operatorname{PtdIns}[4,5] \mathrm{P}_{2}$ concentration $(1-6 \mathrm{~mol} \%)$ was investigated. Network morphologies were evaluated to obtain quantitative parameters describing the density, the height and the filament length of the MAC. Using two different methods, SOAX and an artificial retina, the obtained fluorescence micrographs of network morphologies were skeletonized, A comparison of both methods demonstrated that the artificial retina is better suited to skeletonize the fluorescence micrographs. Utilizing these network skeletons, it could be shown that the height of the MAC is independent of the provided connection points, while the density given by the node density and mesh size, increased with increasing connection points. Interestingly, during the attachment process a filament sorting effect was observed. The filament contour length observed in membrane attached MACs was significantly shorter than those of the preformed 3D F-actin network gels. Furthermore, the filament length was even decreased, if more connection points were provided. Diffusion coefficients of the underlying lipid bilayer were not significantly affected by the attachment of the F-actin network.

To investigate the mechanical properties of the lipid bilayer attached MAC, video particle tracking microrheology was performed. The overall stiffness of the F-actin network was characterized by the plateau modulus $G_{0}$ and showed a 15 -fold increase in contrast to 3D F-actin network gels. Increasing the number of membrane 
connections, increased the overall stiffness. This result implies that the rheological properties of the MAC is governed by the dynamic attachment of the network to the lipid bilayer. By means of indentation experiments the change in lateral membrane tension, induced by F-actin attachment, was investigated. It could be shown that the attachment of an F-actin network alters the lateral membrane tension; however, the influence of the network was strongly dependent on the morphology of the network.

In the next step, the influence of cross-linking actin binding proteins (ABP) on the MAC architecture was investigated. The model system is highly suitable to characterize influences of only one protein, in contrast to cellular cortices, in which different ABPs are present and redundancies between the different APBs result. In this work, the influence of fascin, a bundling protein present in filopodia structures, and $\alpha$-actinin, a prevailed cross-linking protein, on MACs was investigated. Preformed MACs were treated with ABPs in situ. Both ABPs showed a rearrangement of the MAC: fascin bundled the actin network, which resulted in an almost 2.5-fold increase in filament contour length and a decreasing node density of the cortex. In case of $\alpha$-actinin bundling and recruiting of the F-actin network was observed, showing large spider web-like structures formed on top of the flat F-actin cortex. This demonstrates that MACs physiologically attached via ezrin are dynamic enough to investigate the biological functions of single ABPs. 


\section{Bibliography}

[1] V. A. Consuelas, D. J. Minas: Actin: Structure, functions, and disease, Nova Science Publishers, New York, 2012.

[2] B. Alberts, A. Johnson, J. Lewis, M. Raff, K. Roberts, P. Walter: Molecular Biology of the Cell; 5. Edition, Garland Science, New York, 2008.

[3] F. Huber, A. Boire, M. P. López, G. H. Koenderink: Cytoskeletal crosstalk, Curr. Opin. Cell Biol., 2015, 32, 39-47.

[4] R. Sanghvi-Shah, G. F. Weber: Intermediate Filaments at the Junction of Mechanotransduction, Migration, and Development, Front. Cell Dev. Biol., 2017, 5,81 .

[5] L. Blanchoin, R. Boujemaa-Paterski, C. Sykes, J. Plastino: Actin dynamics, architecture, and mechanics in cell motility, Physiol. Rev., 2014, 94 (1), 235-263.

[6] T. D. Pollard, J. A. Cooper: Actin, a central player in cell shape and movement, Science, 2009, 326 (5957), 1208-1212.

[7] M. Vicente-Manzanares, X. Ma, R. S. Adelstein, A. R. Horwitz: Non-muscle myosin II takes centre stage in cell adhesion and migration, Nat. Rev. Mol. Cell Biol., 2009, 10 (11), 778-790.

[8] E. Reisler, E. H. Egelman: Actin structure and function, J. Biol. Chem., 2007, 282 (50), 36133-36137.

[9] B. J. Perrin, J. M. Ervasti: The actin gene family, Cytoskeleton (Hoboken), 2010, 67 (10), 630-634.

[10] L. R. Otterbein, P. Graceffa, R. Dominguez [last visited 17.07.2018], Uncomplexed Actin - PDB entry: 1J6Z, retrieved from https://www.rcsb.org/structure/1j6z, 2001.

[11] P. Graceffa, R. Dominguez: Crystal structure of monomeric actin in the ATP state. Structural basis of nucleotide-dependent actin dynamics, J. Biol. Chem., 2003, 278 (36), 34172-34180.

[12] E. M. de La Cruz, A. Mandinova, M. O. Steinmetz, D. Stoffler, U. Aebi, T. D. Pollard: Polymerization and structure of nucleotide-free actin filaments, J. Mol. Biol., 2000, 295 (3), 517-526.

[13] H. Schüler, M. Nyåkern, C. E. Schutt, U. Lindberg, R. Karlsson: Mutational analysis of arginine 177 in the nucleotide binding site of $\beta$-actin, Eur. J. Biochem., 2000, 267 (13), 4054-4062. 
[14] M.-F. Carlier, D. Pantaloni, E. Korn, Edward D.: The Mechanism of ATP Hydrolysis Accompanying the Polymerization of Mg-actin and Ca-actin, J. Biol. Chem., 1987, 262 (7), 3052-3059.

[15] Y. Doi, C. Frieden: Actin Polymerization, J. Biol. Chem., 1984, 259 (17), 1186811875 .

[16] E. Korn, M.-F. Carlier, D. Pantaloni: Actin Polymerization and ATP Hydrolysis, Science, 1987, 238, 638-644.

[17] T. D. Pollard, L. Blanchoin, R. D. Mullins: Molecular Mechanisms Controlling Actin Filament Dynamics in Nonmuscle Cells, Annu. Rev. Biophys. Biomol. Struct., 2000, 29, 545-576.

[18] Y. L. Wang: Exchange of actin subunits at the leading edge of living fibroblasts, J. Cell Biol., 1985, 101 (2), 597-602.

[19] T. D. Pollard: Regulation of actin filament assembly by Arp2/3 complex and formins, Annu. Rev. Biophys. Biomol. Struct., 2007, 36, 451-477.

[20] T. Niedermayer, A. Jégou, L. Chièze, B. Guichard, E. Helfer, G. Romet-Lemonne, M.-F. Carlier, R. Lipowsky: Intermittent depolymerization of actin filaments is caused by photo-induced dimerization of actin protomers, Proc. Natl. Acad. Sci. USA, 2012, 109 (27), 10769-10774.

[21] R. Dominguez, K. C. Holmes: Actin structure and function, Annu. Rev. Biophys., 2011, 40, 169-186.

[22] J. Hanson, J. Lowy: The structure of F-actin and of actin filaments isolated from muscle, J. Mol. Biol., 1963, 6 (1), 46-IN5.

[23] T. Fujii, A. H. Iwane, T. Yanagida, K. Namba: Direct visualization of secondary structures of F-actin by electron cryomicroscopy, Nature, 2010, 467 (7316), 724728.

[24] E. Grazi: What is the diameter of the actin filament?, FEBS Lett., 1997, 405 (3), 249-252.

[25] J. A. Cooper: Effects of cytochalasin and phalloidin on actin, J. Cell Biol., 1987, 105 (4), 1473-1478.

[26] A. M. Lengsfeld, I. Löw, T. Wieland, P. Dancker, W. Hasselbach: Interaction of Phalloidin with Actin, Proc. Natl. Acad. Sci. USA, 1974, 74 (7), 2803-2807.

[27] P. Dancker, I. Löw, W. Hasselbach, T. Wieland: Interaction of Actin with Phalloidin: Polymerization and Stabilization of F-Actin, Biochim. Biophys. Acta, 1975, 400, 407-414. 
[28] J. E. Estes, L. A. Selden, L. C. Gershman: Mechanism of action of phalloidin on the polymerization of muscle actin, Biochemistry, 2002, 20 (4), 708-712.

[29] Y. Miyamoto, M. Kuroda, E. Munekata, T. Masaki: Stoichiometry of Actin and Phalloidin Binding: One Molecule of the Toxin Dominates Two Actin Subunits, J. Biochem., 1986, 100, 1677-1680.

[30] T. Oda, K. Namba, Y. Maéda: Position and orientation of phalloidin in F-actin determined by X-ray fiber diffraction analysis, Biophys. J., 2005, 88 (4), 2727 2736 .

[31] T. Yanagida, M. Nakase, K. Nishiyama, F. Oosawa: Direct observation of motion of single F-actin filaments in the presence of myosin, Nature, 1984, 307, 58-60.

[32] D. Vignjevic, S.-I. Kojima, Y. Aratyn, O. Danciu, T. Svitkina, G. G. Borisy: Role of fascin in filopodial protrusion, J. Cell Biol., 2006, 174 (6), 863-875.

[33] K. Türmer, J. Orbán, P. Gróf, M. Nyitrai: FASCIN and alpha-actinin can regulate the conformation of actin filaments, Biochim. Biophys. Acta, 2015, 1850 (9), $1855-1861$.

[34] P. K. Mattila, P. Lappalainen: Filopodia, Nat. Rev. Mol. Cell Biol., 2008, 9 (6), $446-454$

[35] T. P. Stossel, J. Condeelis, L. Cooley, J. H. Hartwig, A. Noegel, M. Schleicher, S. S. Shapiro: Filamins as integrators of cell mechanics and signalling, Nat. Rev. Mol. Cell Biol., 2001, 2 (2), 138-145.

[36] D. Laporte, N. Ojkic, D. Vavylonis, J.-Q. Wu: $\alpha$-Actinin and fimbrin cooperate with myosin II to organize actomyosin bundles during contractile-ring assembly, Mol. Biol. Cell, 2012, 23 (16), 3094-3110.

[37] A.-C. Reymann, R. Boujemaa-Paterski, J.-L. Martiel, C. Guérin, W. Cao, H. F. Chin, E. M. de La Cruz, M. Théry, L. Blanchoin: Actin network architecture can determine myosin motor activity, Science, 2012, 336 (6086), 1310-1314.

[38] M. Pring, A. Weber, and M. R. Bubb: Profilin-actin complexes directly elongate actin filaments at the barbed end, Biochemistry, 1992, 31, 1827-1836.

[39] E. Prochniewicz, Q. Zhang, P. A. Janmey, D. D. Thomas: Cooperativity in FActin: Binding of Gelsolin at the Barbed End Affects Structure and Dynamics of the Whole Filament, J. Mol. Biol., 1996, 260, 756-766.

[40] R. D. Mullins, J. A. Heuser, T. D. Pollard: The interaction of Arp2 $/ 3$ complex with actin: Nucleation, high affinity pointed end capping, and formation of branching networks of filaments, Proc. Natl. Acad. Sci. USA, 1998, 95, 61816186 . 
[41] L. M. Machesky, R. H. Insall: Scar1 and the related Wiskott-Aldrich syndrome protein, WASP, regulate the actin cytoskeleton through the Arp2/3 complex, Curr. Biol., 1998, 8 (25), 1347-1356.

[42] J. Block, D. Breitsprecher, S. Kühn, M. Winterhoff, F. Kage, R. Geffers, P. Duwe, J. L. Rohn, B. Baum, C. Brakebusch et al.: FMNL2 drives actin-based protrusion and migration downstream of Cdc42, Curr. Biol., 2012, 22 (11), 1005-1012.

[43] P. Chugh, A. G. Clark, M. B. Smith, D. A. D. Cassani, K. Dierkes, A. Ragab, P. P. Roux, G. Charras, G. Salbreux, E. K. Paluch: Actin cortex architecture regulates cell surface tension, Nat. Cell Biol., 2017, 19 (6), 689-697.

[44] N. Morone, T. Fujiwara, K. Murase, R. S. Kasai, H. Ike, S. Yuasa, J. Usukura, A. Kusumi: Three-dimensional reconstruction of the membrane skeleton at the plasma membrane interface by electron tomography, J. Cell Biol., 2006, 174 (6), $851-862$.

[45] A. Bretscher, K. Edwards, R. G. Fehon: ERM proteins and merlin, Nat. Rev. Mol. Cell Biol., 2002, 3 (8), 586-599.

[46] S. Tsukita, S. Yonemura: Cortical Actin Organization: Lessons form ERM (Ezrin/Radixin/Moesin) Proteins, J. Biol. Chem., 1999, 274 (49), 34507-34510.

[47] A. Bretscher, D. Reczek, M. Berryman: Ezrin: a protein requiring conformational activation to link microfilaments to the plasma membrane in the assembly of cell surface structures, J. Cell Sci., 1997, 110, 3011-3018.

[48] A. Bretscher: Purification of an 80,000-dalton protein that is a component of the isolated microvillus cytoskeleton, and its localization in nonmuscle cells, J. Cell Biol., 1983, 97 (2), 425-432.

[49] O. Turunen: Ezrin has a COOH-terminal actin-binding site that is conserved in the ezrin protein family, J. Cell Biol., 1994, 126 (6), 1445-1453.

[50] R. G. Fehon, A. I. McClatchey, A. Bretscher: Organizing the cell cortex, Nat. Rev. Mol. Cell Biol., 2010, 11 (4), 276-287.

[51] S. Bosk, J. A. Braunger, V. Gerke, C. Steinem: Activation of F-actin binding capacity of ezrin, Biophys. J., 2011, 100 (7), 1708-1717.

[52] B. T. Fievet, A. Gautreau, C. Roy, L. Del Maestro, P. Mangeat, D. Louvard, M. Arpin: Phosphoinositide binding and phosphorylation act sequentially in the activation mechanism of ezrin, J. Cell Biol., 2004, 164 (5), 653-659.

[53] R. Gary, A. Bretscher: Ezrin self-association involves binding of an N-terminal domain to a normally masked C-terminal domain that includes the F-actin binding site, Mol. Biol. Cell, 1995, 6 (8), 1061-1075. 
[54] D. N. Chambers, A. Bretscher: Ezrin mutants affecting dimerization and activation, Biochemistry, 2005, 44 (10), 3926-3932.

[55] J. A. Braunger, B. R. Brückner, S. Nehls, A. Pietuch, V. Gerke, I. Mey, A. Janshoff, C. Steinem: Phosphatidylinositol 4,5-bisphosphate alters the number of attachment sites between ezrin and actin filaments, J. Biol. Chem., 2014, 289 (14), 9833-9843.

[56] V. Shabardina, C. Kramer, B. Gerdes, J. Braunger, A. Cordes, J. Schäfer, I. Mey, D. Grill, V. Gerke, C. Steinem: Mode of Ezrin-Membrane Interaction as a Function of PIP2 Binding and Pseudophosphorylation, Biophys. J., 2016, 110 (12), 2710-2719.

[57] R. Zhou, L. Zhu, A. Kodani, P. Hauser, X. Yao, J. G. Forte: Phosphorylation of ezrin on threonine 567 produces a change in secretory phenotype and repolarizes the gastric parietal cell, J. Cell Sci., 2005, 118 Pt 19, 4381-4391.

[58] J. M. Berg, J. L. Tymoczko, L. Stryer: Biochemie; 6. Edition, Spektrum Akademischer Verlag, Heidelberg, 2010.

[59] S. McLaughlin, J. Wang, A. Gambhir, D. Murray: PIP(2) and proteins, Annu. Rev. Biophys. Biomol. Struct., 2002, 31, 151-175.

[60] J. E. Ferrell: Phosphoinositide metabolism and the morphology of human erythrocytes, J. Cell Biol., 1984, 98 (6), 1992-1998.

[61] G. R. V. Hammond: Does PtdIns(4,5)P2 concentrate so it can multi-task?, Biochem. Soc. Trans., 2016, 44 (1), 228-233.

[62] F. Fernandes, L. M. S. Loura, A. Fedorov, M. Prieto: Absence of clustering of phosphatidylinositol-(4,5)-bisphosphate in fluid phosphatidylcholine, J. Lipid Res., 2006, 47 (7), 1521-1525.

[63] E. G. Tall, I. Spector, S. N. Pentyala, I. Bitter, M. J. Rebecchi: Dynamics of phosphatidylinositol 4,5-bisphosphate in actin-rich structures, Curr. Biol., 2000, $10(12), 743-746$.

[64] M. Schön, I. Mey, C. Steinem: Influence of cross-linkers on ezrin-bound minimal actin cortices, Prog. Biophys. Mol. Biol., 2018, in press, DOI: https://doi.org/10.1016/j.pbiomolbio.2018.07.016.

[65] E. Abu Shah, K. Keren: Symmetry breaking in reconstituted actin cortices, eLife, 2014, 3, e01433.

[66] D. V. Köster, K. Husain, E. Iljazi, A. Bhat, P. Bieling, R. D. Mullins, M. Rao, S. Mayor: Actomyosin dynamics drive local membrane component organization in 
an in vitro active composite layer, Proc. Natl. Acad. Sci. USA, 2016, 113 (12), E1645-54.

[67] J. D. Cortese, B. Schwab, C. Frieden, E. L. Elson: Actin polymerization induces a shape change in actin-containing vesicles, Proc. Natl. Acad. Sci. USA, 1989, $86(15), 5773-5777$.

[68] W. Häckl, M. Bärmann, E. Sackmann: Shape Changes of Self-Assembled Actin Bilayer Composite Membranes, Phys. Rev. Lett., 1998, 80 (8), 1786-1789.

[69] L. Limozin, E. Sackmann: Polymorphism of cross-linked actin networks in giant vesicles, Phys. Rev. Lett., 2002, 89 (16), 168103.

[70] A. L. Hitt: Ponticulin is the major high affinity link between the plasma membrane and the cortical actin network in Dictyostelium, J. Cell Biol., 1994, 126 (6), 1433-1444.

[71] V. Papayannopoulos, C. Co, K. E. Prehoda, S. Snapper, J. Taunton, W. A. Lim: A polybasic motif allows N-WASP to act as a sensor of PIP(2) density, Mol. Cell, 2005, 17 (2), 181-191.

[72] L.-L. Pontani, J. van der Gucht, G. Salbreux, J. Heuvingh, J.-F. Joanny, C. Sykes: Reconstitution of an actin cortex inside a liposome, Biophys. J., 2009, 96 (1), 192-198.

[73] A. P. Liu, D. L. Richmond, L. Maibaum, S. Pronk, P. L. Geissler, D. A. Fletcher: Membrane-induced bundling of actin filaments, Nat. Phys., 2008, 4, 789-793.

[74] D. Ershov, M. C. Stuart, J. van der Gucht: Mechanical properties of reconstituted actin networks at an oil-water interface determined by microrheology, Soft Matter, 2012, 8 (21), 5896.

[75] E. Helfer, S. Harlepp, L. Bourdieu, J. Robert, F. C. MacKintosh, D. Chatenay: Microrheology of biopolymer-membrane complexes, Phys. Rev. Lett., 2000, 85 (2), 457-460.

[76] E. Helfer, S. Harlepp, L. Bourdieu, J. Robert, F. C. MacKintosh, D. Chatenay: Buckling of actin-coated membranes under application of a local force, Phys. Rev. Lett., 2001, 87 (8), 88103.

[77] E. Helfer, S. Harlepp, L. Bourdieu, J. Robert, F. C. MacKintosh, D. Chatenay: Viscoelastic properties of actin-coated membranes, Phys. Rev. E, 2001, $632 \mathrm{Pt}$ 1,21904 .

[78] E. A. Evans: Bending Resistance and Chemically Induced Moments in Membrane Bilayers, Biophys. J., 1974, 14 (12), 923-931. 
[79] V. Caorsi, J. Lemière, C. Campillo, M. Bussonnier, J. Manzi, T. Betz, J. Plastino, K. Carvalho, C. Sykes: Cell-sized liposome doublets reveal active tension buildup driven by acto-myosin dynamics, Soft Matter, 2016, 12 (29), 6223-6231.

[80] A. P. Liu, D. A. Fletcher: Actin polymerization serves as a membrane domain switch in model lipid bilayers, Biophys. J., 2006, 91 (11), 4064-4070.

[81] D. Chereau, F. Kerff, P. Graceffa, Z. Grabarek, K. Langsetmo, R. Dominguez: Actin-bound structures of Wiskott-Aldrich syndrome protein (WASP)-homology domain 2 and the implications for filament assembly, Proc. Natl. Acad. Sci. USA, 2005, 102 (46), 16644-16649.

[82] S. K. Vogel, Z. Petrasek, F. Heinemann, P. Schwille: Myosin motors fragment and compact membrane-bound actin filaments, eLife, 2013, 2:e0016, 1-18.

[83] S. K. Vogel, P. Schwille: Minimal systems to study membrane-cytoskeleton interactions, Curr. Opin. Biotechnol., 2012, 23 (5), 758-765.

[84] R. W. Friddle, A. Noy, J. J. de Yoreo: Interpreting the widespread nonlinear force spectra of intermolecular bonds, Proc. Natl. Acad. Sci. USA, 2012, 109 (34), $13573-13578$.

[85] A. Holmberg, A. Blomstergren, O. Nord, M. Lukacs, J. Lundeberg, M. Uhlén: The biotin-streptavidin interaction can be reversibly broken using water at elevated temperatures, Electrophoresis, 2005, 26 (3), 501-510.

[86] M. Janke, A. Herrig, J. Austermann, V. Gerke, C. Steinem, A. Janshoff: Actin binding of ezrin is activated by specific recognition of PIP2-functionalized lipid bilayers, Biochemistry, 2008, 47 (12), 3762-3769.

[87] G. Lee, D. A. Kidwell, R. J. Colton: Sensing Discrete Streptavidin-Biotin Interactions with Atomic Force Microscopy, Langmuir, 1994, 10 (2), 354-357.

[88] U. Piran, W. J. Riordan: Dissociation rate constant of the biotin-streptavidin complex, J. Immunol. Methods, 1990, 133 (1), 141-143.

[89] M. P. Murrell, M. L. Gardel: F-actin buckling coordinates contractility and severing in a biomimetic actomyosin cortex, Proc. Natl. Acad. Sci. USA, 2012, 109 (51), 20820-20825.

[90] M. Murrell, M. L. Gardel: Actomyosin sliding is attenuated in contractile biomimetic cortices, Mol. Biol. Cell, 2014, 25 (12), 1845-1853.

[91] C. T. Skau, D. S. Courson, A. J. Bestul, J. D. Winkelman, R. S. Rock, V. Sirotkin, D. R. Kovar: Actin filament bundling by fimbrin is important for endocytosis, cytokinesis, and polarization in fission yeast, J. Biol. Chem., 2011, 286 (30), 26964-26977. 
[92] C. M. Hampton, D. W. Taylor, K. A. Taylor: Novel structures for alpha-actinin:Factin interactions and their implications for actin-membrane attachment and tension sensing in the cytoskeleton, J. Mol. Biol., 2007, 368 (1), 92-104.

[93] K. Wang, S. J. Singer: Interaction of filamin with f-actin in solution, Proc. Natl. Acad. Sci. USA, 1977, 74 (5), 2021-2025.

[94] A. C. Martin, M. Kaschube, E. F. Wieschaus: Pulsed contractions of an actinmyosin network drive apical constriction, Nature, 2009, 457 (7228), 495-499.

[95] I. Linsmeier, S. Banerjee, P. W. Oakes, W. Jung, T. Kim, M. P. Murrell: Disordered actomyosin networks are sufficient to produce cooperative and telescopic contractility, Nat. Commun., 2016, 7, 12615.

[96] R. J. Barfoot, K. H. Sheikh, B. R. G. Johnson, J. Colyer, R. E. Miles, L. J. C. Jeuken, R. J. Bushby, S. D. Evans: Minimal F-actin cytoskeletal system for planar supported phospholipid bilayers, Langmuir, 2008, 24 (13), 6827-6836.

[97] B. R. Lentz, Y. Barenholz, T. E. Thompson: Fluorescence depolarization studies of phase transitions and fluidity in phospholipid bilayers, Biochemistry, 1976, 15 (20), 4521-4528.

[98] Avanti Polar Lipids Inc. [last visited 03.01.2018], Brain PI(4,5)P2 / 840046, retrieved from https://avantilipids.com/product/840046/, 2018.

[99] C. R. Benatti, M. T. Lamy, R. M. Epand: Cationic amphiphiles and the solubilization of cholesterol crystallites in membrane bilayers, Biochim. Biophys. Acta, 2008, 1778 (4), 844-853.

[100] M. Ramezani, M. Koshhamdam, A. Dehshahri, B. Malaekeh-Nikouei: The influence of size, lipid composition and bilayer fluidity of cationic liposomes on the transfection efficiency of nanolipoplexes, Colloids Surf. B Biointerfaces, 2009, $72(1), 1-5$.

[101] R. B. Campbell, S. V. Balasubramanian, R. M. Straubinger: Phospholipid-cationic lipid interactions: influences on membrane and vesicle properties, Biochim. Biophys. Acta, 2001 (1512), 27-39.

[102] D. Afanasenkau, A. Offenhäusser: Positively charged supported lipid bilayers as a biomimetic platform for neuronal cell culture, Langmuir, 2012, 28 (37), 1338713394.

[103] S. Iwabuchi, T. Takahashi, K. Hatori: Transport of actin-decorated liposomes along myosin molecules in vitro, Biochem. Biophys. Res. Commun., 2012, 422 (1), 164-168. 
[104] J. W. Dubendorff, F. W. Studier: Controlling Basal Expression in an Inducible T7 Expression System by Blocking the Target T7 Promoter with lac Repressor, J. Mol. Biol., 1991, 219, 45-59.

[105] Machery-Nagel GmbH \& Co. KG: NucleoBond Xtra Endotoxin-Free Plasmid DNA Purification User Manual, 2014, 1-53.

[106] A. Herrig, M. Janke, J. Austermann, V. Gerke, A. Janshoff, C. Steinem: Cooperative adsorption of ezrin on PIP2-containing membranes, Biochemistry, 2006, 45 (43), 13025-13034.

[107] V. Gaberc-Porekar, V. Menart: Perspectives of immobilized-metal affinity chromatography, J. Biochem. Biophys. Methods, 2001, 49 1-3, 335-360.

[108] J. Porath, J. A.N. Carlsson, I. Olsson, G. Belfrage: Metal chelate affinity chromatography, a new approach to protein fractionation, Nature, 1975, 258 (5536), 598-599.

[109] U. K. Laemmli: Cleavage of Structural Proteins during the Assembly of the Head of Bacteriophage T4, Nature, 1970, 227, 680-686.

[110] Y. Kim, S. O. Ho, N. R. Gassman, Y. Korlann, E. V. Landorf, F. R. Collart, S. Weiss: Efficient site-specific labeling of proteins via cysteines, Bioconjugate Chem., 2008, 19 (3), 786-791.

[111] D. P. Nair, M. Podgórski, S. Chatani, T. Gong, W. Xi, C. R. Fenoli, C. N. Bowman: The Thiol-Michael Addition Click Reaction, Chem. Mater., 2013, 26 (1), 724-744.

[112] M. I. Angelova, D. S. Dimitrov: Liposome Electroformation, Faraday Discuss. Chem. Soc., 1986, 81, 303-311.

[113] R. Dimova, K. A. Riske, S. Aranda, N. Bezlyepkina, R. L. Knorr, R. Lipowsky: Giant vesicles in electric fields, Soft Matter, 2007, 3 (7), 817.

[114] L. A. Bagatolli, T. Parasassi, E. Gratton: Giant phospholipid vesicles: comparison among the whole lipid sample characteristics using different preparation methods, Chem. Phys. Lipids, 2000, 105, 135-147.

[115] C. Herold, G. Chwastek, P. Schwille, E. P. Petrov: Efficient electroformation of supergiant unilamellar vesicles containing cationic lipids on ITO-coated electrodes, Langmuir, 2012, 28 (13), 5518-5521.

[116] C. Kramer: Verknüpfung zwischen Plasmamembran und Zytoskelett: Charakterisierung der Organisation von Ezrin und F-Aktin an artifiziellen Lipidmembranen, PhD thesis, University of Göttingen, 2016. 
[117] Strauss, Georg, N., S. Schlichtherle, H. K. Pulker [last visited 23.06.2018], Verdampfen, Aufdampfen, Kondensieren., retrieved from http://www.phystechcoating.com/pdf/PhysTech-2005-1-Verdampfen.pdf, 2005.

[118] C. D. Bain, E. B. Troughton, Y.-T. Tao, J. Evall, G. M. Whitesides, R. G. Nuzzo: Formation of monolayer films by the spontaneous assembly of organic thiols from solution onto gold, J. Am. Chem. Soc., 1989, 111 (1), 321-335.

[119] J. A. Braunger, C. Kramer, D. Morick, C. Steinem: Solid supported membranes doped with PIP2, Langmuir, 2013, 29 (46), 14204-14213.

[120] G. Gauglitz: Multiple reflectance interference spectroscopy measurements made in parallel for binding studies, Rev. Sci. Instrum., 2005, 76 (6), 62224.

[121] I. Langmuir, V. J. Schäfer, D. M. Wrinch: Built-up films of proteins and their properties, Science, 1937, 85 (2194), 76-80.

[122] G. Gauglitz: Interferometry in Bio- and Chemosensing, Optical Chemical Sensors; 1. Edition, Springer, 2006.

[123] R. C. Weast (Hrsg.) CRC handbook of chemistry and physics A ready-reference book of chemical and physical data, CRC Pr, Cleveland, Ohio, 1975.

[124] G. Vuye, S. Fisson, V. Nguyen Van, Y. Wang, J. Rivory, F. Abelès: Temperature dependence of the dielectric function of silicon using in situ spectroscopic ellipsometry, Thin Solid Films, 1993, 233 1-2, 166-170.

[125] P. Schiebener, J. Straub, J. M. H. Levelt Sengers, J. S. Gallagher: Refractive index of water and steam as function of wavelength, temperature and density, $J$. Phys. Chem. Ref. Data, 1990, 19 (3), 677-717.

[126] G. Ghosh: Dispersion-equation coefficients for the refractive index and birefringence of calcite and quartz crystals, Opt. Commun., 1999, 163 1-3, 95102 .

[127] J. Vörös: The density and refractive index of adsorbing protein layers, Biophys. J., 2004, 87 (1), 553-561.

[128] H.-M. Schmitt, A. Brecht, J. Piehler, G. Gauglitz: An integrated system for optical biomolecular interaction analysis, Biosens. Bioelectron., 1997, 12 (8), 809-816.

[129] C. DeCusatis, C. A. MacDonald, E. W. van Stryland, G. Li, J. M. Enoch, M. Bass, V. Lakshminarayanan, V. N. Mahajan: Handbook of Optics: Volume I: Geometrical and physical optics, polarized light, components and instruments; 3. Edition, McGraw-Hill, New York, Optical Society of America, 2010. 
[130] M. Stephan: Development of a Biomembrane Sensor Based on Reflectometry, PhD thesis, University of Göttingen, 2013.

[131] S. Bosk: Dynamische Strukturen am Zellcortex: Aktivierbarkeit und Akkumulation von Ezrin in Abhängigkeit von PIP2, PhD thesis, University of Göttingen, 2011.

[132] ExPASy Bioinformatics Resource Portal [last visited 23.04.2018], ProtParam Tool. Ezrin (Accession Number: P15311), retrieved from https://web.expasy.org $\lfloor$ protparam/, 2018.

[133] Atto-Tec GmbH [last visited 02.05.2018], Product Information: Atto 488, retrieved from http://www.atto-tec.com/fileadmin/user_upload/Katalog Flyer_Support/ATTO_488.pdf, 2017.

[134] G. Binnig, C. F. Quate, and Ch. Gerber: Atomic Force Microscope, Phys. Rev. Lett., 1986, 56 (9), 930-934.

[135] N. Jalili, K. Laxminarayana: A review of atomic force microscopy imaging systems, Mechatronics (Oxf), 2004, 14 (8), 907-945.

[136] Bruker AFM Probes [last visited 11.07.2018], MLCT - Product Description, retrieved from https://www.brukerafmprobes.com/p-3444-mlct.aspx, 2018.

[137] J. L. Hutter, J. Bechhoefer: Calibration of atomic- force microscope tips, Rev. Sci. Instrum., 1993, 64 (7), 1868-1873.

[138] J. E. Sader, J. W. M. Chon, P. Mulvaney: Calibration of rectangular atomic force microscope cantilevers, Rev. Sci. Instrum., 1999, 70 (10), 3967-3969.

[139] I. P. Mey: Elastizität porenüberspannender Membranen: Eine kraftmikroskopische Studie, $P h D$ thesis, Johannes Gutenberg-Universität Mainz, 2009.

[140] JPK Instruments AG [last visited 11.07.2018], JPK Instruments Technical Note: QI mode - Quantitative Imaging with the NanoWizard 3, retrieved from https:// www.jpk.com/app-technotes-img/AFM/pdf/jpk-tech-quantitative-imaging.141.pdf, 2014.

[141] H.-J. Butt, B. Cappella, M. Kappl: Force measurements with the atomic force microscope, Surf. Sci. Rep., 2005, 59 1-6, 1-152.

[142] J. W. Lichtman, J.-A. Conchello: Fluorescence microscopy, Nat. Methods, 2005, 2 (12), 910-919.

[143] Carl Zeiss Microscopy GmbH [last visited 24.05.2018], The Confocal Principle, retrieved from https://www.zeiss.com/microscopy/int/products/confocalmicroscopes/lsm-800-for-materials.html, 2018. 
[144] J.-A. Conchello, J. W. Lichtman: Optical sectioning microscopy, Nat. Methods, 2005, 2 (12), 920-931.

[145] H. Kirshner, F. Aguet, D. Sage, M. Unser: 3-D PSF fitting for fluorescence microscopy, J. Microsc., 2013, 249 (1), 13-25.

[146] M. Gleisner: Interaction of Epsin N-Terminal Homology domain (ENTH) with artificial lipid membranes as a function of lateral tension, $P h D$ thesis, University of Göttingen, 2016.

[147] R. W. Cole, T. Jinadasa, C. M. Brown: Measuring and interpreting point spread functions to determine confocal microscope resolution and ensure quality control, Nat. Protoc., 2011, 6 (12), 1929-1941.

[148] Carl Zeiss Microscopy GmbH in SpringerReference, Springer-Verlag, Berlin/Heidelberg, 2011.

[149] K. Weisshart [last visited 10.07.2018], Zeiss Technology Note: The Basic Principle of Airyscanning, retrieved from http://confocal-club.ru/upload/article/EN_wp LSM-880_Basic-Principle-Airyscan.pdf, 2014.

[150] Carl Zeiss Microscopy GmbH [last visited 10.07.2018], Beam Path - ZeisS Airyscan, retrieved from https://applications.zeiss.com/C125792900358A3F/0/ 99BD4F163C8B84E4C1257DF500558F63/\$FILE/EN $41 \quad 012$ _095_AiryscanUpgrade_rel-1_0.pdfhttps://applications.zeiss.com/C125792900358A3F /0/ B3454621FFCC99C7C1257DCE005151E1/\$FILE/EN_41_050_014_LSM800Airyscan_Beam-Path 50x70 lowres.pdf, 2015.

[151] J. Huff, W. Bathe, R. Netz, T. Anhut, K. Weisshart [last visited 10.07.2018], The Airyscan Detector form Zeiss, retrieved from https://applications.zeiss.com/ C125792900358A3F/0/BF16BECDC08849E1C1257ED60029AF8D/\$FILE/EN $41 \quad 013 \quad 105 \quad$ wp $\quad$ Airyscan-detector.pdf, 2015.

[152] C. J. R. Sheppard, S. B. Mehta, R. Heintzmann: Superresolution by image scanning microscopy using pixel reassignment, Opt. Lett., 2013, 38 (15), 28892892 .

[153] R. Engelmann, K. Weisshart, B. Zimmermann, J. Birkenbeil, J. Tham [last visited 10.07.2018], Zeiss Technology Note: Airyscanning, retrieved from https:// applications.zeiss.com/C125792900358A3F /0/ 4833AA1630EDFB71C1257D3A004E497D/\$FILE/EN $41 \quad 013 \quad 085 \quad$ LSM880 Airyscanning.pdf, 2014.

[154] C. B. Müller, J. Enderlein: Image scanning microscopy, Phys. Rev. Lett., 2010, 104 (19), 198101. 
[155] A. G. York, P. Chandris, D. D. Nogare, J. Head, P. Wawrzusin, R. S. Fischer, A. Chitnis, H. Shroff: Instant super-resolution imaging in live cells and embryos via analog image processing, Nat. Methods, 2013, 10 (11), 1122-1126.

[156] A. G. York, S. H. Parekh, D. Dalle Nogare, R. S. Fischer, K. Temprine, M. Mione, A. B. Chitnis, C. A. Combs, H. Shroff: Resolution doubling in live, multicellular organisms via multifocal structured illumination microscopy, Nat. Methods, 2012, $9(7), 749-754$.

[157] D. Axelrod, D. E. Koppel, J. Schlessinger, E. Elson, W. W. Webb: Mobility measurement by analysis of fluorescence photobleaching recovery kinetics, Biophys. J., 1976, 16 (9), 1055-1069.

[158] S. Seiffert, W. Oppermann: Systematic evaluation of FRAP experiments performed in a confocal laser scanning microscope, J. Microsc., 2005, $220 \mathrm{Pt} 1$, $20-30$.

[159] P. Jönsson, M. P. Jonsson, J. O. Tegenfeldt, F. Höök: A method improving the accuracy of fluorescence recovery after photobleaching analysis, Biophys. J., 2008, 95 (11), 5334-5348.

[160] H. Nöding, M. Schön, C. Reinermann, N. Dörrer, A. Kürschner, B. Geil, I. Mey, C. Heussinger, A. Janshoff, C. Steinem: Rheology of Membrane-Attached Minimal Actin Cortices, J. Phys. Chem. B, 2018, 122 (16), 4537-4545.

[161] G. Gonzales, F. Fleuret, P. Fua: Learning Rotational Features for Filament Detection, IEEE Conf. Comput. Vis. Pattern Recognit., 2009, 1582-1589.

[162] T. Xu, D. Vavylonis, F.-C. Tsai, G. H. Koenderink, W. Nie, E. Yusuf, L. I-Ju, J.Q. Wu, X. Huang: SOAX: A software for quantification of 3D biopolymer networks, Sci. Rep., 2015, 5, 9081.

[163] T. Xu, D. Vavylonis, X. Huang: 3D actin network centerline extraction with multiple active contours, Med. Image Anal., 2014, 18 (2), 272-284.

[164] M. Molteni, D. Magatti, B. Cardinali, M. Rocco, F. Ferri: Fast two-dimensional bubble analysis of biopolymer filamentous networks pore size from confocal microscopy thin data stacks, Biophys. J., 2013, 104 (5), 1160-1169.

[165] S. Münster, B. Fabry: A simplified implementation of the bubble analysis of biopolymer network pores, Biophys. J., 2013, 104 (12), 2774-2775.

[166] H. Nöding: Active and Passive Microrheology of F-Actin Membrane Composites: From Minimal Cortex Model Systems to Living Cells, PhD thesis, University of Göttingen, 2017. 
[167] M. Kilfoil [last visited 27.05.2017], 2D feature finding and tracking algorithms \& microrheology algorithms. MATLAB code, retrieved from http:// people.umass.edu/kilfoil/tools.php, 2017.

[168] B. R. Dasgupta, S.-Y. Tee, J. C. Crocker, B. J. Frisken, D. A. Weitz: Microrheology of polyethylene oxide using diffusing wave spectroscopy and single scattering, Phys. Rev. E, 2002, 655 Pt 1, 51505.

[169] T. G. Mason, K. Ganesan, J. H. van Zanten, D. Wirtz, S. C. Kuo: Particle Tracking Microrheology of Complex Fluids, Phys. Rev. Lett., 1997, 79 (17), 32823285 .

[170] D. C. Morse: Viscoelasticity of Concentrated Isotropic Solutions of Semiflexible Polymers. 1. Model and Stress Tensor, Macromolecules, 1998, 31 (20), 7030-7043.

[171] D. C. Morse: Viscoelasticity of Concentrated Isotropic Solutions of Semiflexible Polymers. 2. Linear Response, Macromolecules, 1998, 31 (20), 7044-7067.

[172] F. Gittes, F. C. MacKintosh: Dynamic shear modulus of a semiflexible polymer network, Phys. Rev. E, 1998, 58 (2), R1241-R1244.

[173] J. Plagge, A. Fischer, C. Heussinger: Viscoelasticity of reversibly crosslinked networks of semiflexible polymers, Phys. Rev. E, 2016, 93 (6), 62502.

[174] Addgene Inc. [last visited 20.06.2018], List of sequencing pimers, retrieved from https://www.addgene.org/mol-bio-reference/sequencing-primers/, 2018.

[175] ExPASy Bioinformatics Resource Portal [last visited 12.04.2018], Protparam Translate Tool DNA to AA, retrieved from https://web.expasy.org/translate/, 2018.

[176] The UniProt Consortium [last visited 23.04.2018], Amino Acid Sequence of Ezrin, retrieved from https://www.uniprot.org/uniprot/P15311, 2017.

[177] A. Herrig: Wechselwirkung von Ezrin mit PIP2-haltigen artifiziellen Membransystemen und mit F-Aktin, PhD thesis, University of Regensburg, 2007.

[178] J. A. Braunger: Ezrin activation in vitro: Investigation of ezrin's conformation and the interaction between ezrin and F-actin, PhD thesis, University of Göttingen, 2013.

[179] K. L. Gould, J. A. Cooper, A. Bretscher, T. Hunter: The protein-tyrosine kinase substrate, p81, is homologous to a chicken microvillar core protein, J. Cell Biol., 1986, 102 (2), 660-669.

[180] K. L. Gould, A. Bretscher, F. S. Esch, T. Hunter: cDNA cloning and sequencing of the protein-tyrosine kinase substrate, ezrin, reveals homology to band 4.1, EMBO J., 1989, 8 (13), 4133-4142. 
[181] O. Turunen, R. Winqvist, R. Pakkanen, K.-H. Grzeschik, T. Wahlström, A. Vaheri: Cytovillin, a Microvillar Mr 75000 Protein, J. Biol. Chem., 1989, 264 (28), 16727-16732.

[182] V. N. Balaji, M. J. Rao, S. N. Rao, S. W. Dietrich, V. Sasisekharan: Geometry of Proline and Hydroxyproline, Biochem. Biophys. Res. Commun., 1986, 140 (3), 895-900.

[183] J. M. Phang, S. J. Harrop, A. P. Duff, A. V. Sokolova, B. Crossett, J. C. Walsh, S. A. Beckham, C. D. Nguyen, R. B. Davies, C. Glöckner et al.: Structural characterization suggests models for monomeric and dimeric forms of full-length ezrin, Biochem. J., 2016, 473 (18), 2763-2782.

[184] I. Santa Cruz Biotechnology [last visited 29.05.2018], PIP2 (CAS 383907-42-4), retrieved from https://www.scbt.com/scbt/product/pip2-383907-42-4, 2018.

[185] Z. Salamon, G. Tollin: Optical Anisotropy in Lipid Bilayer Membranes:, Biophys. J., 2001, 80, 1557-1567.

[186] D. Boal: Mechanics of the Cell, Cambridge University Press, Cambridge, 2012.

[187] N. Kučerka, M.-P. Nieh, J. Katsaras: Fluid phase lipid areas and bilayer thicknesses of commonly used phosphatidylcholines as a function of temperature, Biochim. Biophys. Acta, 2011, 1808 (11), 2761-2771.

[188] S. Boussaad, J. Pean, N. J. Tao: High-Resolution Multiwavelength Surface Plasmon Resonance Spectroscopy for Probing Conformational and Electronic Changes in Redox Proteins, Anal. Chem., 2000, 72 (1), 222-226.

[189] R. Naumann, E. K. Schmidt, A. Jonczyk, K. Fendler, B. Kadenbach, T. Liebermann, A. Offenhäusser, W. Knoll: The peptide-tethered lipid membrane as a biomimetic system to incorporate cytochrome c oxidase in a functionally active form, Biosens. Bioelectron., 1999, 14 (7), 651-662.

[190] Q. Li, M. R. Nance, R. Kulikauskas, K. Nyberg, R. Fehon, P. A. Karplus, A. Bretscher, J. J. G. Tesmer: Self-masking in an intact ERM-merlin protein, J. Mol. Biol., 2007, 365 (5), 1446-1459.

[191] J. J. Jayasundar, J. H. Ju, L. He, D. Liu, F. Meilleur, J. Zhao, D. J. E. Callaway, Z. Bu: Open conformation of ezrin bound to phosphatidylinositol 4,5-bisphosphate and to F-actin revealed by neutron scattering, J. Biol. Chem., 2012, 287 (44), 37119-37133.

[192] G. Blin, E. Margeat, K. Carvalho, C. A. Royer, C. Roy, C. Picart: Quantitative analysis of the binding of ezrin to large unilamellar vesicles containing phosphatidylinositol 4,5 bisphosphate, Biophys. J., 2008, 94 (3), 1021-1033. 
[193] J. M. Phang, S. J. Harrop, A. P. Duff, A. V. Sokolova, B. Crossett, J. C. Walsh, S. A. Beckham, C. D. Nguyen, R. B. Davies, C. Glöckner et al.: Structural characterization suggests models for monomeric and dimeric forms of full-length ezrin, Biochem. J., 2016, 473 (18), 2763-2782.

[194] GeneCards Human Gene Database [last visited 02.05.2018], GAPDH Gene (Protein Coding), retrieved from http://www.genecards.org/cgi-bin/ carddisp.pl?gene=GAPDH, 2018.

[195] E. Limpert, W. A. Stahel, M. Abbt: Log-normal Distributions across the Sciences: Keys and Clues, BioScience, 2001, 51 (5), 341-352.

[196] F. Gittes: Flexural rigidity of microtubules and actin filaments measured from thermal fluctuations in shape, J. Cell Biol., 1993, 120 (4), 923-934.

[197] J. Käs, H. Strey, J. X. Tang, D. Finger, R. Ezzell, E. Sackmann, P. A. Janmey: F-actin, a model polymer for semiflexible chains in dilute, semidilute, and liquid crystalline solutions, Biophys. J., 1996, 70 (2), 609-625.

[198] D. Mazia: Adhesion of cells to surfaces coated with polylysine. Applications to electron microscopy, J. Cell Biol., 1975, 66 (1), 198-200.

[199] K. S. Kim, K. H. Lee, K. Cho, C. E. Park: Surface modification of polysulfone ultrafiltration membrane by oxygen plasma treatment, J. Memb. Sci., 2002, 199 $1-2,135-145$.

[200] F. Heinemann, S. K. Vogel, P. Schwille: Lateral membrane diffusion modulated by a minimal actin cortex, Biophys. J., 2013, 104 (7), 1465-1475.

[201] K. Murase, T. Fujiwara, Y. Umemura, K. Suzuki, R. Iino, H. Yamashita, M. Saito, H. Murakoshi, K. Ritchie, A. Kusumi: Ultrafine membrane compartments for molecular diffusion as revealed by single molecule techniques, Biophys. J., 2004, 86 (6), 4075-4093.

[202] R. Iino, I. Koyama, A. Kusumi: Single Molecule Imaging of Green Fluorescent Proteins in Living Cells: E-Cadherin Forms Oligomers on the Free Cell Surface, Biophys. J., 2001, 80, 2667-2677.

[203] T. Xu, H. Li, T. Shen, N. Ojkic, D. Vavylonis, X. Huang: Extraction and analysis of actin networks based on Open Active Contour models, IEEE Int. Symp. Biomed. Imaging, 2011, 1334-1340.

[204] F. Gittes, B. Mickey, J. Nettleton, J. Howard: Flexural Rigidity of Microtubules and Actin Filaments Measured from Thermal Fluctuations in Shape, J. Cell Biol., 1993, 4 (120), 923-934. 
[205] M. Fritzsche, C. Erlenkämper, E. Moeendarbary, G. Charras, K. Kruse: Actin kinetics shapes cortical network structure and mechanics, Sci. Adv., 2016, 2 (4), e1501337.

[206] A. Kishino, T. Yanagida: Force measurements by micromanipulation of a single actin filament by glass needles, Nature, 1988, 334, 74-76.

[207] M. Fritzsche, D. Li, H. Colin-York, V. T. Chang, E. Moeendarbary, J. H. Felce, E. Sezgin, G. Charras, E. Betzig, C. Eggeling: Self-organizing actin patterns shape membrane architecture but not cell mechanics, Nat. Commun., 2017, 8, 14347.

[208] M. Fritzsche: Self-organizing actin patterns shape cytoskeletal cortex organization, Commun. Integr. Biol., 2017, 10 (3), e1303591.

[209] M. T. Valentine, Z. E. Perlman, M. L. Gardel, J. H. Shin, P. Matsudaira, T. J. Mitchison, D. A. Weitz: Colloid surface chemistry critically affects multiple particle tracking measurements of biomaterials, Biophys. J., 2004, 86 (6), 40044014 .

[210] M. L. Gardel, M. T. Valentine, J. C. Crocker, A. R. Bausch, D. A. Weitz: Microrheology of entangled F-actin solutions, Phys. Rev. Lett., 2003, 91 (15), 158302.

[211] O. Lieleg, M. M. A. E. Claessens, A. R. Bausch: Structure and dynamics of crosslinked actin networks, Soft Matter, 2010, 6 (2), 218-225.

[212] D. A. Head, A. J. Levine, F. C. MacKintosh: Distinct regimes of elastic response and deformation modes of cross-linked cytoskeletal and semiflexible polymer networks, Phys. Rev. E, 2003, 686 Pt 1, 61907.

[213] J. Xu, A. Palmer, D. Wirtz: Rheology and Microrheology of Semiflexible Polymer Solutions: Actin Filament Networks, Macromolecules, 1989, 31, 6486-6492.

[214] J. Hildebrandt: Comparison of mathematical models for cat lung and viscoelastic balloon derived by laplace transform methods from pressurevolume data, Bull. Math. Biophys., 1969, 31 (4), 651-667.

[215] O. Lieleg, M. M. A. E. Claessens, Y. Luan, A. R. Bausch: Transient binding and dissipation in cross-linked actin networks, Phys. Rev. Lett., 2008, 101 (10), 108101.

[216] P. A. Janmey, K. Iida, H. L. Yin, T. P. Stossel: Polyphosphoinositide Micelles and Polyphosphoinositide-containing Vesicles Dissociate Endogenous GelsolinActin Complexes and Promote Actin Assembly from the Fast-growing End of Actin Filaments Blocked by Gelsolin, J. Biol. Chem., 1987, 262 (25), 1222812236 . 
[217] A. M. McGough, C. J. Staiger, J.-K. Min, K. D. Simonetti: The gelsolin family of actin regulatory proteins, FEBS Lett., 2003, 552 2-3, 75-81.

[218] P. A. Janmey, S. Hvidt, J. Peetermans, J. Lamb, J. D. Ferry, T. P. Stossel: Viscoelasticity of F-actin and F-actin/gelsolin complexes, Biochemistry, 2002, 27 (21), 8218-8227.

[219] M. Fritzsche, R. Thorogate, G. Charras: Quantitative analysis of ezrin turnover dynamics in the actin cortex, Biophys. J., 2014, 106 (2), 343-353.

[220] S. B. Marston: The rates of formation and dissociation of actin-myosin complexes, Biochem. J., 1982, 203, 453-460.

[221] O. Lieleg, K. M. Schmoller, M. M. A. E. Claessens, A. R. Bausch: Cytoskeletal polymer networks, Biophys. J., 2009, 96 (11), 4725-4732.

[222] S. M. V. Ward, A. Weins, M. R. Pollak, D. A. Weitz: Dynamic viscoelasticity of actin cross-linked with wild-type and disease-causing mutant alpha-actinin-4, Biophys. J., 2008, 95 (10), 4915-4923.

[223] N. M. Green: The use of [14C]Biotin for kinetic studies and for assay, Biochem. J., 1963, 89, 585-591.

[224] P. Vermette, T. Gengenbach, U. Divisekera, P. A. Kambouris, H. J. Griesser, L. Meagher: Immobilization and surface characterization of NeutrAvidin biotinbinding protein on different hydrogel interlayers, J. Colloid Interface Sci., 2003, 259 (1), 13-26.

[225] Polysciences Inc. [last visited 15.06.2018], The Benefits of Neutravidin over Streptavidin and Avidin. Technical Data Sheet 779, retrieved from http:// polysciences.com/skin/frontend/default/polysciences/pdf/779.pdf, 2008.

[226] R. Walder, A. J. Levine, M. Dennin: Rheology of two-dimensional F-actin networks associated with a lipid interface, Phys. Rev. E, 2008, 771 Pt 1, 11909.

[227] L. S. Jung, K. E. Nelson, P. S. Stayton, C. T. Campbell: Binding and Dissociation Kinetics of Wild-Type and Mutant Streptavidins on Mixed Biotin-Containing Alkylthiolate Monolayers, Langmuir, 2000, 16 (24), 9421-9432.

[228] A. T. Martilla, O. H. Laitinen, K. J. Airenne, T. Kulik, E. A. Bayer, M. Wilchek, M. S. Kulomaa: Recombinant NeutraLite Avidin: a nonglycosylated, acidic mutant of chicken avidin that exhibits high affinity for biotin and low nonspecific binding properties, FEBS Lett., 2000, 467, 31-36.

[229] S. Zhao, W. M. Reichert: Influence of biotin lipid surface density and accessibility on avidin binding to the tip of an optical fiber sensor, Langmuir, 1992, 8 (11), 2785-2791. 
[230] J. R. Wayment, J. M. Harris: Biotin-avidin binding kinetics measured by singlemolecule imaging, Anal. Chem., 2009, 81 (1), 336-342.

[231] S. Guo, C. Ray, A. Kirkpatrick, N. Lad, B. B. Akhremitchev: Effects of multiplebond ruptures on kinetic parameters extracted from force spectroscopy measurements, Biophys. J., 2008, 95 (8), 3964-3976.

[232] C.-K. Lee, Y.-M. Wang, L.-S. Huang, S. Lin: Atomic force microscopy, Micron, 2007, 38 (5), 446-461.

[233] M. Kocun, T. D. Lazzara, C. Steinem, A. Janshoff: Preparation of solvent-free, pore-spanning lipid bilayers, Langmuir, 2011, 27 (12), 7672-7680.

[234] I. Mey, M. Stephan, E. K. Schmitt, M. M. Müller, M. Ben Amar, C. Steinem, A. Janshoff: Local membrane mechanics of pore-spanning bilayers, J. Am. Chem. Soc., 2009, 131 (20), 7031-7039.

[235] S. Steltenkamp, M. M. Müller, M. Deserno, C. Hennesthal, C. Steinem, A. Janshoff: Mechanical properties of pore-spanning lipid bilayers probed by atomic force microscopy, Biophys. J., 2006, 91 (1), 217-226.

[236] E. Dulkeith, A. C. Morteani, T. Niedereichholz, T. A. Klar, J. Feldmann, S. A. Levi, F. C. J. M. van Veggel, D. N. Reinhoudt, M. Möller, D. I. Gittins: Fluorescence quenching of dye molecules near gold nanoparticles, Phys. Rev. Lett., 2002, 89 (20), 203002.

[237] M. Böcker, S. Muschter, E. K. Schmitt, C. Steinem, T. E. Schäffer: Imaging and patterning of pore-suspending membranes with scanning ion conductance microscopy, Langmuir, 2009, 25 (5), 3022-3028.

[238] F. W. Putnam: The plasma proteins: Structure, function, and genetic control. Volume I; 2. Edition, Academic Press, New York, 1975.

[239] Sigma-Aldrich [last visited 30.05.2018], BSA - Product Information Sheet, retrieved from https://www.sigmaaldrich.com/content/dam/sigma-aldrich/docs/ Sigma/Product_Information_Sheet/b4287pis.pdf, 2000.

[240] J. W. Kuhlmann, I. P. Mey, C. Steinem: Modulating the lateral tension of solventfree pore-spanning membranes, Langmuir, 2014, 30 (27), 8186-8192.

[241] U. Komaragiri, M. R. Begley, J. G. Simmonds: The Mechanical Response of Freestanding Circular Elastic Films Under Point and Pressure Loads, J. Appl. Mech., 2005, 72 (2), 203.

[242] E. Evans, W. Rawicz: Entropy-driven tension and bending elasticity in condensed-fluid membranes, Phys. Rev. Lett., 1990, 64 (17), 2094-2097. 
[243] D. Needham, R. M. Hochmut: Electro-mechanical permeabilization of lipid vesicles, Biophys. J., 1989, 55, 1001-1009.

[244] E. Evans, D. Needham: Physical properties of surfactant bilayer membranes: thermal transitions, elasticity, rigidity, cohesion and colloidal interactions, $J$. Phys. Chem., 1987, 91 (16), 4219-4228.

[245] D. Needham, R. S. Nunn: Elastic deformation and failure of lipid bilayer membranes containing cholesterol, Biophys. J., 1990, 58 (4), 997-1009.

[246] D. Norouzi, M. M. Müller, M. Deserno: How to determine local elastic properties of lipid bilayer membranes from atomic-force-microscope measurements, Phys. Rev. E, 2006, 74 (061914), 061914-1 - 061914-12.

[247] N. M. Bhatia, W. Nachbar: Finite indentation of an elastic membrane by a spherical indenter, Int. J. Non Linear Mech., 1968, 3 (3), 307-324.

[248] C.-H. Huang, P.-Y. Hsiao, F.-G. Tseng, S.-K. Fan, C.-C. Fu, R.-L. Pan: Porespanning lipid membrane under indentation by a probe tip, Langmuir, 2011, 27 (19), 11930-11942.

[249] B. Lorenz, I. Mey, S. Steltenkamp, T. Fine, C. Rommel, M. M. Müller, A. Maiwald, J. Wegener, C. Steinem, A. Janshoff: Elasticity mapping of poresuspending native cell membranes, Small, 2009, 5 (7), 832-838.

[250] M. P. Sheetz, J. Dai: Modulation of membrane dynamics and cell motility by membrane tension, Trends Cell Biol., 1996, 6 (3), 85-89.

[251] S. Nehls, A. Janshoff: Elastic Properties of Pore-Spanning Apical Cell Membranes Derived from MDCK II Cells, Biophys. J., 2017, 113 (8), 1822-1830.

[252] J. Larsen, N. S. Hatzakis, D. Stamou: Observation of inhomogeneity in the lipid composition of individual nanoscale liposomes, J. Am. Chem. Soc., 2011, 133 (28), 10685-10687.

[253] N. Teske, J. Sibold, J. Schumacher, N. K. Teiwes, M. Gleisner, I. Mey, C. Steinem: Continuous Pore-Spanning Lipid Bilayers on Silicon Oxide-Coated Porous Substrates, Langmuir, 2017, 33 (49), 14175-14183.

[254] M. D. Porter, T. B. Bright, D. L. Allara, C. E. D. Chidsey: Spontaneously organized molecular assemblies. 4. Structural characterization of n-alkyl thiol monolayers on gold by optical ellipsometry, infrared spectroscopy, and electrochemistry, J. Am. Chem. Soc., 1987, 109 (12), 3559-3568.

[255] M. Honda, K. Takiguchi, S. Ishikawa, H. Hotani: Morphogenesis of liposomes encapsulating actin depends on the type of actin-crosslinking, J. Mol. Biol., 1999, 287 (2), 293-300. 
[256] R. K. Meyer, U. Aebi: Bundling of Actin Filaments by a-actinin Depends on Its Molecular Length, J. Cell Biol., 1990, 110, 2013-2024.

[257] S. Jansen, A. Collins, C. Yang, G. Rebowski, T. Svitkina, R. Dominguez: Mechanism of actin filament bundling by fascin, J. Biol. Chem., 2011, 286 (34), 30087-30096.

[258] J. D. Winkelman, C. Suarez, G. M. Hocky, A. J. Harker, A. N. Morganthaler, J. R. Christensen, G. A. Voth, J. R. Bartles, D. R. Kovar: Fascin- and $\alpha$-ActininBundled Networks Contain Intrinsic Structural Features that Drive Protein Sorting, Curr. Biol., 2016, 26 (20), 2697-2706.

[259] L. M. Machesky, A. Li: Fascin, Commun. Integr. Biol., 2014, 3 (3), 263-270.

[260] M. Vicente-Manzanares, X. Ma, R. S. Adelstein, A. R. Horwitz: Non-muscle myosin II takes centre stage in cell adhesion and migration, Nat. Rev. Mol. Cell Biol., 2009, 10 (11), 778-790.

[261] Y. S. Aratyn, T. E. Schaus, E. W. Taylor, G. G. Borisy: Intrinsic dynamic behavior of fascin in filopodia, Mol. Biol. Cell, 2007, 18 (10), 3928-3940.

[262] D. J. DeRosier, K. T. Edds: Evidence for fascin cross-links between the actin filaments in coelomyocyte, Exp. Cell Res., 1980, 126, 490-494.

[263] N. Kureishy, V. Sapountzi, S. Prag, N. Anilkumar, J. C. Adams: Fascins, and their roles in cell structure and function, BioEssays, 2002, 24 (4), 350-361.

[264] E. S. Chhabra, H. N. Higgs: The many faces of actin: matching assembly factors with cellular structures, Nat. Cell Biol., 2007, 9 (10), 1110-1121.

[265] T. D. Pollard, G. G. Borisy: Cellular Motility Driven by Assembly and Disassembly of Actin Filaments, Cell, 2003, 113 (4), 549.

[266] H. Takatsuki, E. Bengtsson, A. Månsson: Persistence length of fascin-cross-linked actin filament bundles in solution and the in vitro motility assay, Biochim. Biophys. Acta, 2014, 1840 (6), 1933-1942.

[267] M. D. Welch, R. D. Mullins: Cellular control of actin nucleation, Annu. Rev. Cell Dev. Biol., 2002, 18, 247-288.

[268] A. J. Ehrlicher, R. Krishnan, M. Guo, C. M. Bidan, D. A. Weitz, M. R. Pollak: Alpha-actinin binding kinetics modulate cellular dynamics and force generation, Proc. Natl. Acad. Sci. USA, 2015, 112 (21), 6619-6624.

[269] Merck Millipore KGaA [last visited 24.04.2018], 69864 / pET-28a(+) DNA Novagen, retrieved from http://www.merckmillipore.com/DE/en/product/pET28a+-DNA-Novagen,EMD BIO$\underline{69864 \text { RedirectedFrom }=\text { http } \% 3 \mathrm{~A} \% 2 \mathrm{~F} \% 2 \mathrm{Fwww} . \text { emdchemicals.com } \% 2 \text { Flife- }}$ 
6 Bibliography

science-research\%2Fpet-28a-plus-dna\%2FEMD BIO-69864\%2Fp uuid\&attachments=VSEQ, 2018. 


\section{Appendix}

\subsection{Vector Map pET-28a + - Novagen ${ }^{[269]}$}

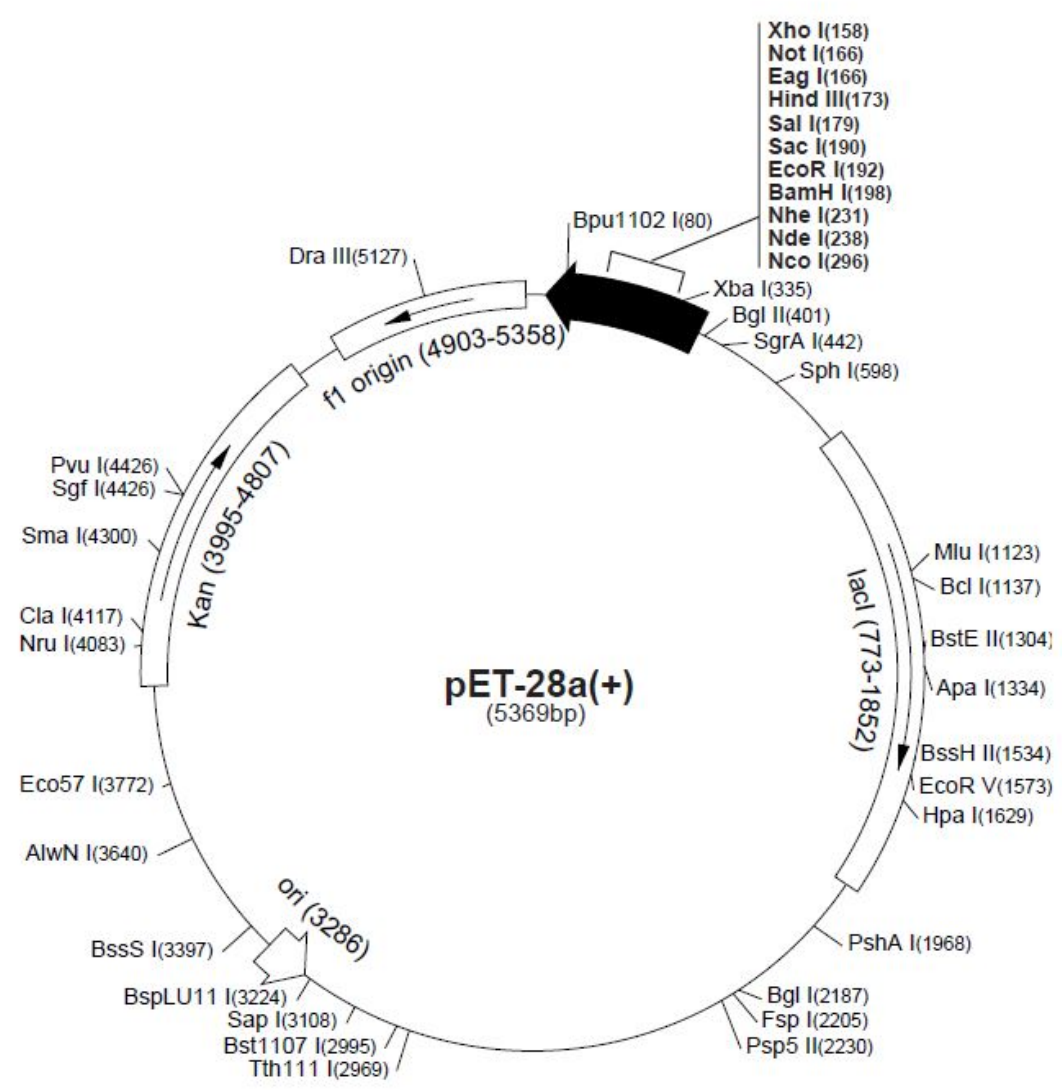




\subsection{Used SOAX Parameters}

Shown parameter set is valid for the input of 8-bit tiff-images.

\begin{tabular}{l|c|c|l|c|c} 
Parameter & $\mathbf{2 D}$ & $\mathbf{3 D}$ & Parameter & $\mathbf{2 D}$ & $\mathbf{3 D}$ \\
\hline intensity-scaling & 0.01 & 0.01 & alpha & 0.01 & 0.01 \\
gaussian standard & 3 & 3 & beta & 0.1 & 0.1 \\
maximum-foreground & 255 & 255 & gamma & 2 & 2 \\
minimum-foreground & 25 & 0 & external-factor & 1 & 1 \\
\hline init z & false & true & stretch-factor & 0.2 & 0.2 \\
\hline snake-point-spacing & 1 & 1 & radial-background-sector & 8 & 8 \\
minimum-snake-length & 5 & 5 & bg-z-xy-ratio & 2.88 & 2.88 \\
\hline maximum-iteration & 10000 & 10000 & radial-near & 4 & 4 \\
change-threshold & 0.05 & 0.05 & radial-far & 8 & 8 \\
\hline check-period & 100 & 100 & delta & 4 & 4 \\
\hline overlap-threshold & 2 & 2 & minimum-linking-angle & 2.1 & 2.1 \\
\hline grouping-distance-thresh & 8 & 8 & damp z & true & false \\
\hline grouping-delta & 8 & 8 & & &
\end{tabular}

\subsection{Used Parameters to fit Rheological Data}

\subsubsection{Parameter Set considering One Cross-Link}

Parameters are: plateau modulus $G_{0}$; cross-over frequency (high frequency branch) $\omega_{0}$; friction coefficients $\eta_{i}$; scaling parameters $\alpha_{0}-\alpha_{1}$.

\begin{tabular}{|c|c|c|c|c|c|}
\hline Sample & $\boldsymbol{G}_{\mathbf{0}} / \mathbf{P a}$ & $\omega_{0} / \mathrm{s}^{-1}$ & $\eta_{1} /$ Pa.s & $\alpha_{0}$ & $\alpha_{1}$ \\
\hline $\begin{array}{c}3 \mathrm{D} \text { actin } \\
(8-23 \mu \mathrm{M}) \\
N=5\end{array}$ & $0.011 \pm 0.0003$ & $0.40 \pm 0.14$ & $1.53 \pm 0.16$ & $1.7 \pm 0.09$ & $0.43 \pm 0.12$ \\
\hline $\begin{array}{c}\text { PtdIns }[4,5] \mathrm{P}_{2} \\
(3 \mathrm{~mol} \%) \\
N=6\end{array}$ & $0.115 \pm 0.004$ & $5.07 \pm 1.71$ & $11.82 \pm 1.3$ & $1.25 \pm 0.13$ & $0.90 \pm 0.32$ \\
\hline $\begin{array}{c}\text { PtdIns }[4,5] \mathrm{P}_{2} \\
(5 \mathrm{~mol} \%) \\
N=9\end{array}$ & $0.221 \pm 0.004$ & $8.14 \pm 1.59$ & $22.86 \pm 1.18$ & $1.16 \pm 0.06$ & $0.90 \pm 0.20$ \\
\hline
\end{tabular}




\subsubsection{Parameter Set considering Two Cross-Links}

Parameters are: plateau modulus $G_{0}$; cross-over frequency (high frequency branch) $\omega_{0}$; friction coefficients $\eta_{i}$; scaling parameters $\alpha_{0}-\alpha_{3}$.

\begin{tabular}{|c|c|c|c|c|}
\hline Sample & $\begin{array}{c}\text { PtdIns }[4,5] P_{2} \\
(3 \operatorname{mol} \%) \\
N=6\end{array}$ & $\begin{array}{c}\text { PtdIns }[4,5] \mathrm{P}_{2} \\
(5 \mathrm{~mol} \%) \\
N=9\end{array}$ & $\begin{array}{c}\text { cap-bio } \\
\text { DOPE } \\
(0.5 \mathrm{~mol} \%) \\
\quad N=1\end{array}$ & $\begin{array}{c}\text { cap-bio } \\
\text { DOPE } \\
(3 \mathrm{~mol} \%) \\
N=4\end{array}$ \\
\hline $\boldsymbol{G}_{\mathbf{0}} / \mathbf{P a}$ & $0.116 \pm 0.007$ & $0.202 \pm 0.013$ & $0.083 \pm 0.011$ & 0.29 \\
\hline$\omega_{0} / \mathrm{s}^{-1}$ & $1.11 \pm 0.81$ & $8.65 \pm 1.02$ & $6.21 \pm 4.28$ & 8.5 \\
\hline$\eta_{1} / \mathbf{P a} \cdot \mathbf{s}$ & $14.03 \pm 0.52$ & $26.45 \pm 0.90$ & $9.54 \pm 1.05$ & 120 \\
\hline$\eta_{1} /$ Pa.s & $3.28 \pm 0.83$ & $6.63 \pm 1.06$ & $1.10 \pm 0.11$ & 3.4 \\
\hline$\alpha_{0}$ & $1.17 \pm 0.09$ & $0.95 \pm 0.07$ & $0.32 \pm 0.20$ & 0.001 \\
\hline$\alpha_{1}$ & $0.16 \pm 0.09$ & $0.80 \pm 0.11$ & $1.23 \pm 0.16$ & 0.85 \\
\hline$\alpha_{2}$ & $0.32 \pm 0.11$ & $0.56 \pm 0.16$ & $1.36 \pm 0.31$ & 0.27 \\
\hline$\alpha_{3}$ & $0.05 \pm 0.06$ & $0.10 \pm 0.07$ & $1.19 \pm 0.29$ & 0.48 \\
\hline
\end{tabular}

The fit for 3 mol \% neutravidin-cap-biotin DOPE binding systems data had to be modeled with a prescribed parameter set. 


\subsection{Used MATLAB Scripts}

\subsubsection{Bubble Mesh Analysis}

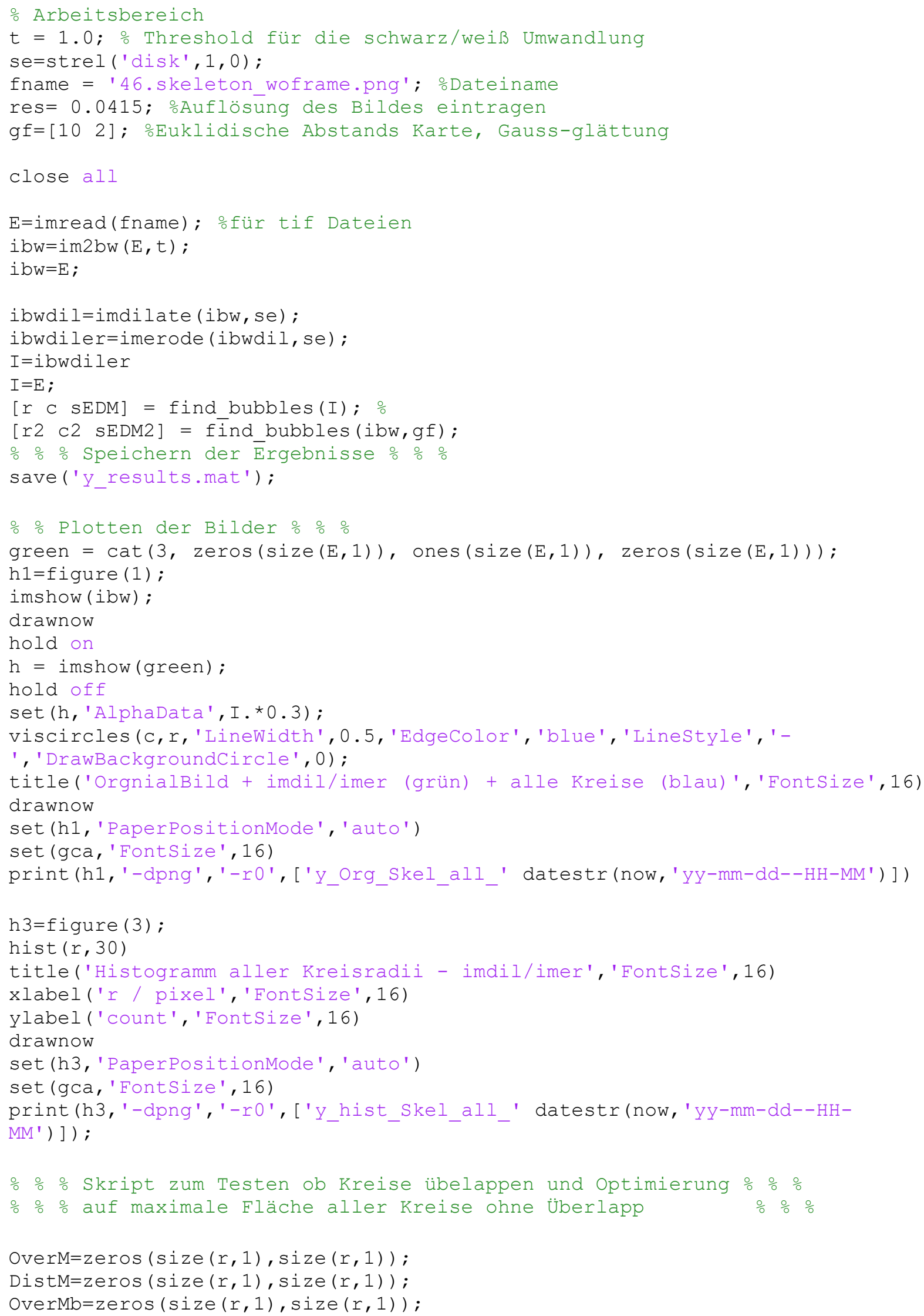




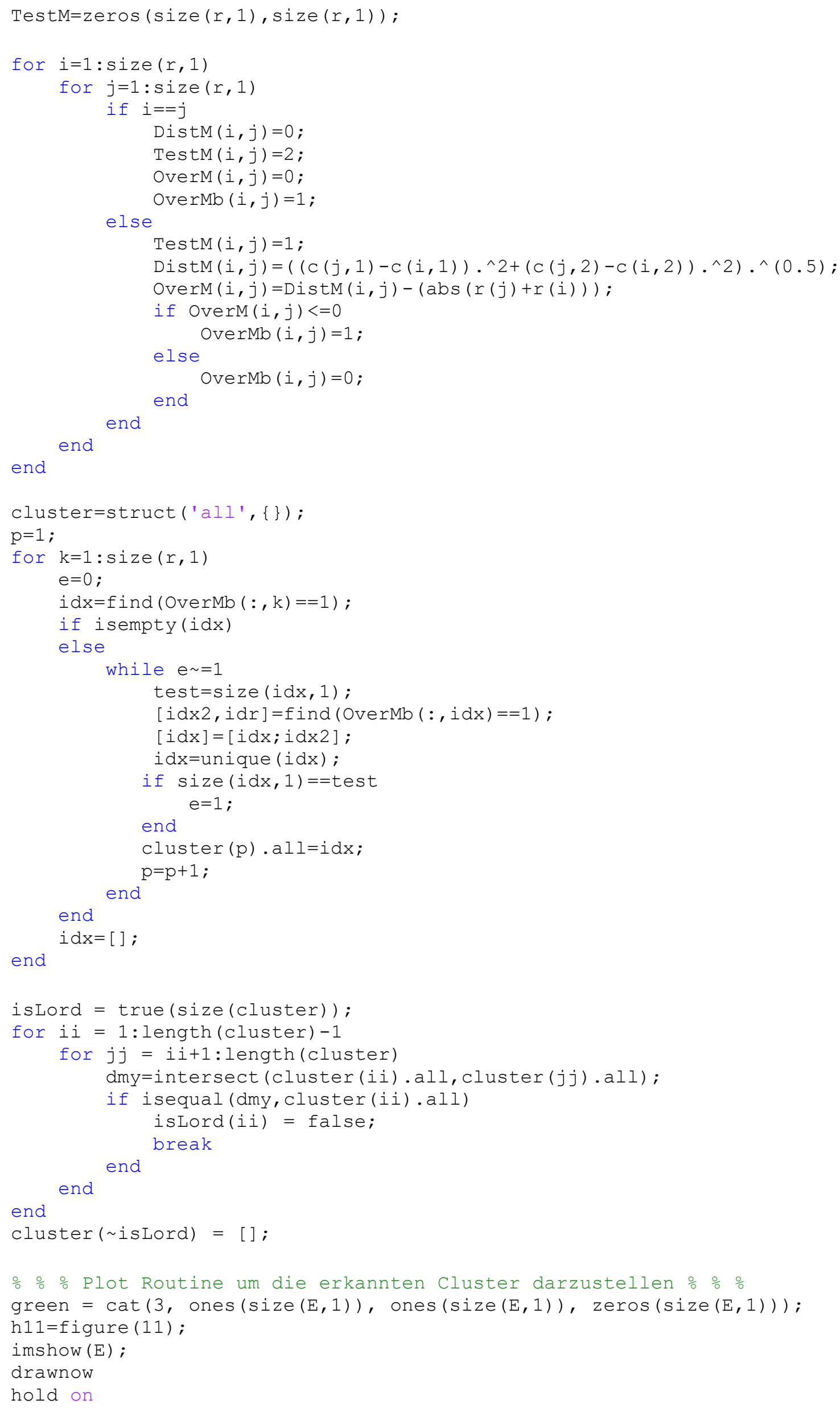




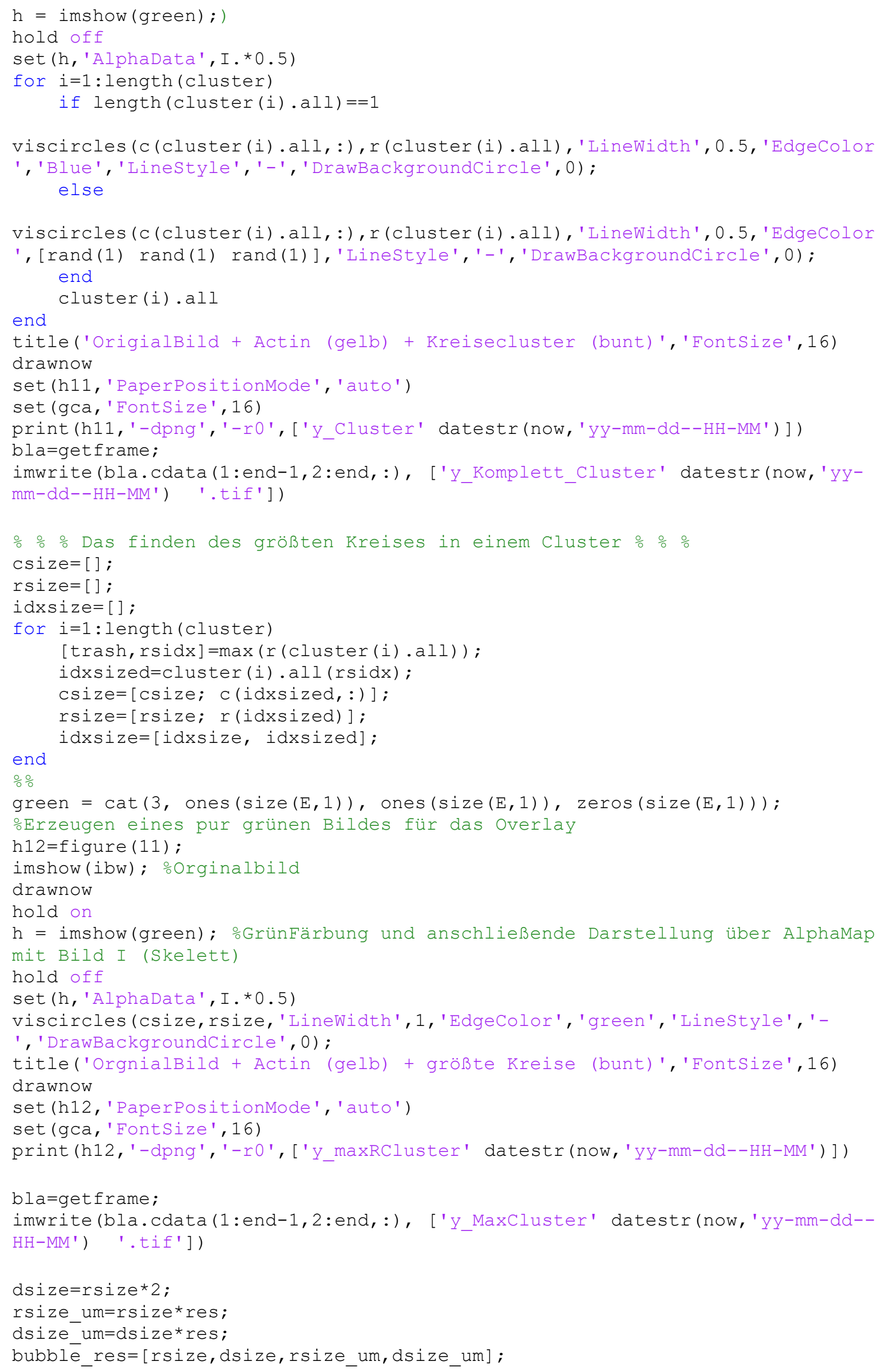




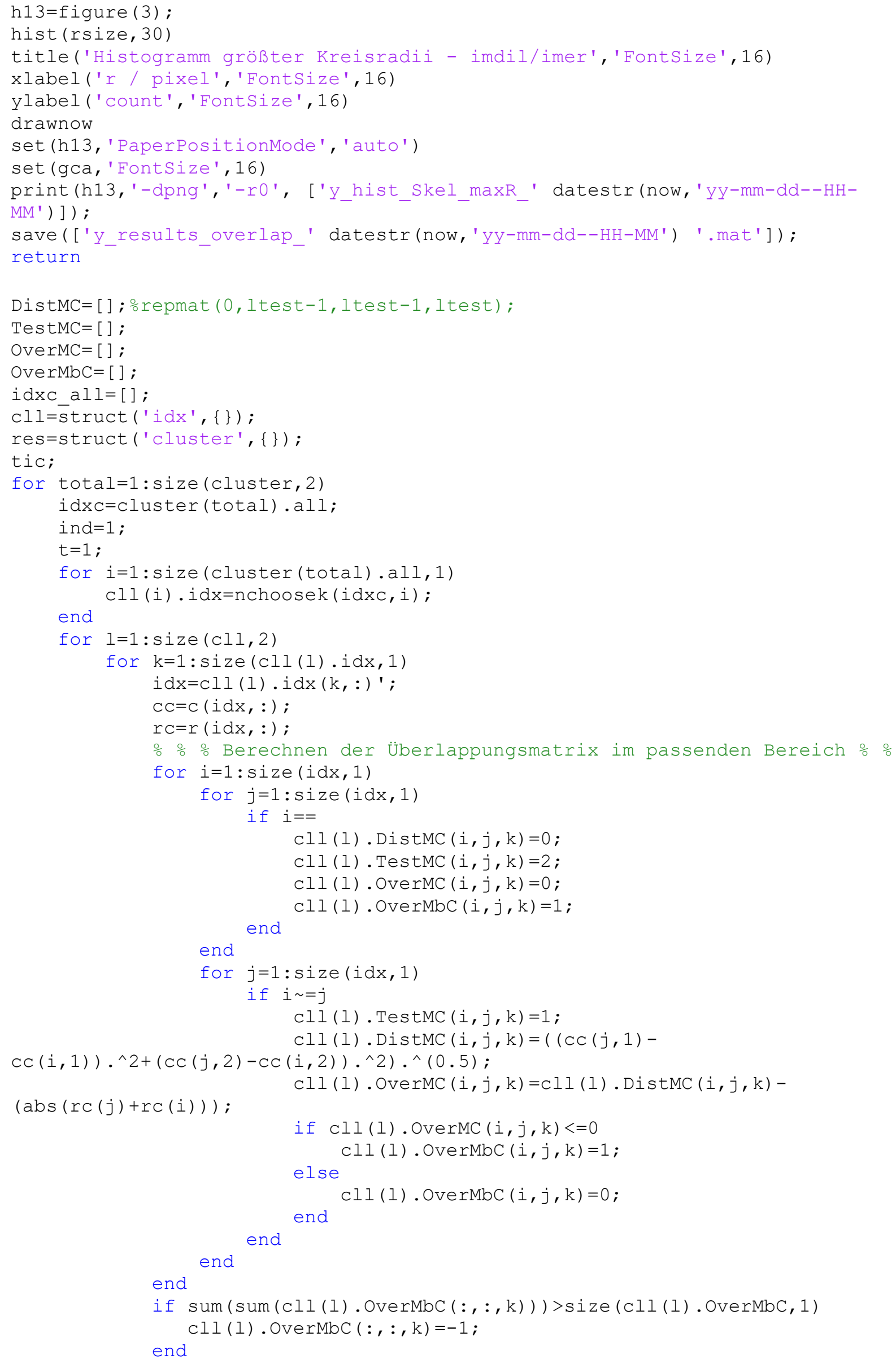


7 Appendix

end

end

for $g=\operatorname{size}(\operatorname{cll}, 2):-1: 1$

for $h=\operatorname{size}(\operatorname{cll}(\mathrm{g}) . i d x, 1):-1: 1$

if $\operatorname{sum}(\operatorname{sum}(\operatorname{cll}(g)$. OverMbC $(:,:, h)))<0$

$\operatorname{cll}(g)$. OverMbC $(:,:, h)=[]$;

$\operatorname{cll}(\mathrm{g})$. OverMC $(:,: \mathrm{h})=[]$;

$\operatorname{cll}(\mathrm{g}) \cdot \operatorname{DistMC}(:,:, \mathrm{h})=[]$;

$\operatorname{cll}(\mathrm{g}) \cdot \operatorname{TestMC}(:,:, \mathrm{h})=[]$;

cll $(g) \cdot i d x(h,:)=[]$;

end

end

end

res (total). cluster $=$ cll;

end

toc; 


\subsection{List of Figures}

Figure 1.1: Localizations of cytoskeletal filaments and regions of their spatial overlaps.[3] 1

Figure 1.2: Actin localizations and organization in the animal cell. ${ }^{[5]}$........................

Figure 1.3: Three conformational states for a single G-actin monomer. ${ }^{[11]}$.................4

Figure 1.4: Helical structure of F-actin derived from cryo-electron microscopy. ${ }^{[21]} \ldots . . .5$

Figure 1.5: Specialized actin organizations and their interplay with several actin binding

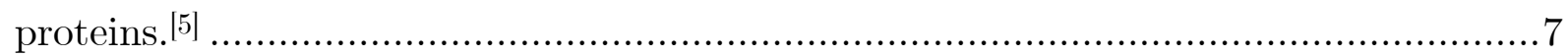

Figure 1.6: Ezrin domain organization and binding model based on a two-step activation process. ${ }^{[50]}$

Figure 1.7: Schematic illustration of different arrangements of minimal actin cortices (MACs). ${ }^{64]}$

Figure 3.1: Single-colonies grown on an agar plate after successful transformation....26

Figure 3.2: Schematic illustration of a GUV electroformation chamber. .36

Figure 3.3: Schematic illustration of PSLB preparation and scanning electron micrographs of a fluXXion $\mathrm{Si}_{3} \mathrm{~N}_{4}$ substrate.

Figure 3.4: Schematic illustration of sedimentation technique. An Eppendorf tip is converted to a buffer column where GUVs were passing through to separate lipid material from vesicles.

Figure 3.5: Schematic illustration of microfluidics chamber and channel construction.

Figure 3.6: Illustration of the optical path in a RIfS setup utilizing Snell's law.......41

Figure 3.7: Schematic illustration of a RIfS measuring chamber........................... 44

Figure 3.8: Schematic illustration of the basic AFM principle. ............................. 47

Figure 3.9: SEM images of MLCT cantilever (A) and cantilever tip (B)................ 48

Figure 3.10: Schematic representation of a typical FDC ................................... 49

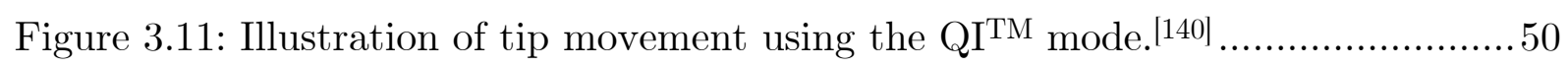

Figure 3.12: Principle of a measurement procedure using the $\mathrm{QI}^{\mathrm{TM}}$ mode............... 51

Figure 3.13: The adjustment cross recorded either by AFM or by fluorescence

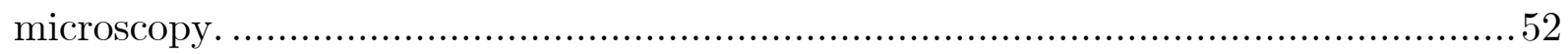

Figure 3.14: Schematic illustration of the principle of Confocal Laser Scanning

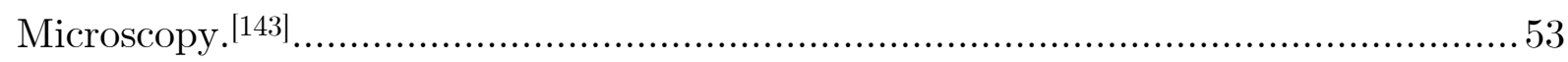

Figure 3.15: Representation of the point-spread-function using the ImageJ plugin PSF

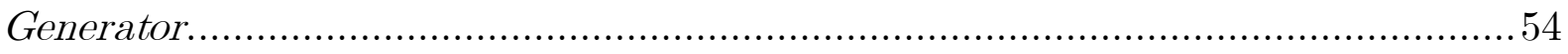

Figure 3.16: Illustration of Airyscan principle.......................................... 56

Figure 3.17: Exemplary comparison of a confocal and an Airyscan image of a typical

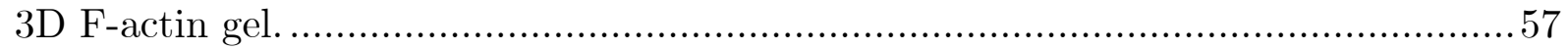

Figure 3.18: Schematically representation of a FRAP experiment........................58 
Figure 3.19: Concept of image processing using the artificial retina analysis. ${ }^{[160]}$ A:..61 Figure 3.20: Exemplary skeletonization of two fluorescence micrographs with different signal-to-noise ratios via the artificial retina. 62

Figure 3.21: Overview of the operating principle of the software SOAX. .63

Figure 3.22: Schematic pixel diagrams of node determination and resulting skeleton with highlighted nodes. 64

Figure 3.23: Important intermediate steps of the mesh size analysis.......................65

Figure 3.24: Overview of video particle tacking microrheology.

Figure 3.25: Schematic illustration of frequency dependent viscoelastic properties of semi-flexible polymers such as F-actin. .68

Figure 4.1: Comparison of determined amino acid sequence (1-letter code) with sequence of UniProt library.[176]

Figure 4.2: Image showing an exemplary SDS-PAGE Gel. ............................. 70

Figure 4.3: Exemplary RIfS diagram showing ezrin T567D binding to a POPC/PtdIns[4,5] $\mathrm{P}_{2}$ (92:8) lipid bilayer.

Figure 4.4: Representation of increasing optical thickness OT caused by ezrin T567D adsorption depending on PtdIns $[4,5] \mathrm{P}_{2}$ content.

Figure 4.5: Physical thickness and relating relative surface coverage of ezrin T567D $(\theta)$ as a function of PtdIns[4,5] $\mathrm{P}_{2}$ content......................................................... 75 Figure 4.6: Threshold analysis of an atomic force micrograph obtained by Shabardina et al. ${ }^{[56]}$ .76

Figure 4.7: Crystallographic structure of $N$-ERMAD showing three subdomains (F1, F2 and F3).[56]

Figure 4.8: Proposed model of ezrin T567D's arrangement on a lipid bilayer doped with $\operatorname{PtdIns}[4,5] \mathrm{P}_{2}$. .78

Figure 4.9: Exemplary UV/Vis spectrum of an elution fraction as outcome of a labeling reaction.

Figure 4.10: Fluorescence micrographs reveal different morphologies of 3D F-actin gels.

82

Figure 4.11: Fluorescence micrograph of a 3D F-actin gel's extension in a microfluidic chamber. .83

Figure 4.12: Determination of F-actin filament length $(L)$ in 3D actin gels by means of SOAX. 84

Figure 4.13: Analysis of F-actin filament thicknesses by means of AFM.[160] .86 Figure 4.14: Fluorescence micrographs of F-actin attachment to an artificial bilayer without receptor lipid. .88

Figure 4.15: Fluorescence micrographs showing an F-actin network attached to an ezrin decorated lipid bilayer. ${ }^{[160]}$ .89 
Figure 4.16: Exemplary network morphologies of attached MACs with different pinning

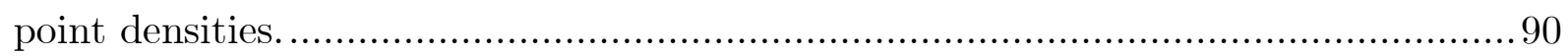

Figure 4.17: Schematic illustration of quantitative network parameters. .................. 92 Figure 4.18: Typical pathway of analyzing a fluorescence micrograph using SOAX (1)

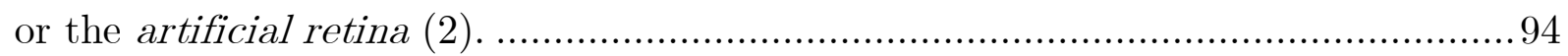

Figure 4.19: Exemplary comparison of the detected SOAX skeleton (A) and the

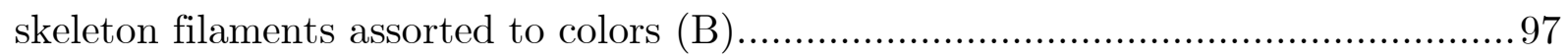
Figure 4.20: Violin plot of the resulting mesh sizes $\xi$ (circle diameter) of network pockets dependent on skeletonization method. .98

Figure 4.21: Box plots representing the node density $\boldsymbol{v}$ (nodes per $\mu^{2}$ ) of network skeletons dependent on the method of skeletonization.

Figure 4.22: Comparison of SOAX's skeleton (A), skeleton of thresholded artificial

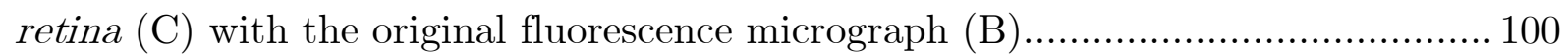

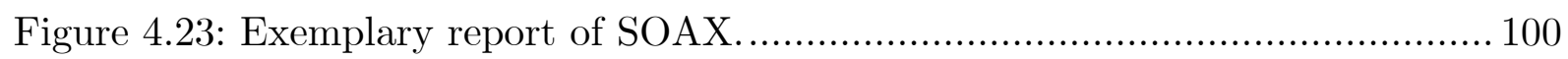

Figure 4.24: Node density $\boldsymbol{v}$ of membrane-attached F-actin networks as a function of the pinning point density. ${ }^{[160]}$ 103

Figure 4.25: Mesh size $\boldsymbol{\xi}$ of membrane-bound F-actin networks as a function of pinning point density represented as relating $\operatorname{PtdIns}[4,5] \mathrm{P}_{2}$ content. 104

Figure 4.26: Exemplary FRAP experiment starting with a time series of fluorescence micrographs. $[160]$ 107

Figure 4.27: Polymerized F-actin networks containing beads with a radius of $1 \mu \mathrm{m}$.

Figure 4.28: Fluorescence micrographs visualize the incorporation of beads into the MAC.

Figure 4.29: Frequency dependent viscoelastic properties of MACs in comparison to 3D F-actin gels. ${ }^{[160]}$

Figure 4.30: Change of the plateau modulus $G_{0}$ of actin model-systems as function of membrane-attachment and further on pinning point density.[160]. 116

Figure 4.31: Frequency dependent viscoelastic properties of 3D F-actin networks with

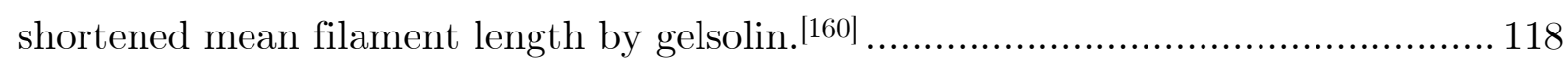

Figure 4.32: Arrhenius plot showing the temperature dependency of the PtdIns $[4,5] \mathrm{P}_{2}$-ezrin-actin binding complex.

Figure 4.33: Frequency dependent viscoelastic properties of biotin-neutravidin mediated, membrane-attached MACs.

Figure 4.34: Differences in spreading behavior and stability of different positively charged receptor lipids. 128

Figure 4.35: Successfully attached MACs to PSLBs (DOPC/DOTAP/TxR-DHPE 79.6:20:0.4) 129 
Figure 4.36: Different F-actin morphologies attached to PSLB with a pore diameter of

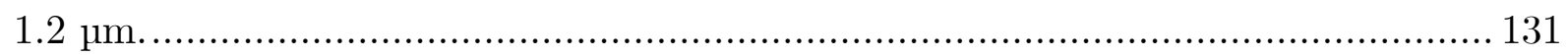

Figure 4.37: Alexa Fluor ${ }^{\circledR}$ 488-phalloidin labeled MACs attached to PSLBs (DOPC/DOTAP/TxR-DHPE (79.6:20:0.4) with a pore diameter of $5.0 \mu \mathrm{m}$. 132

Figure 4.38: Morphologies of found F-actin networks attached to PSLB with a pore

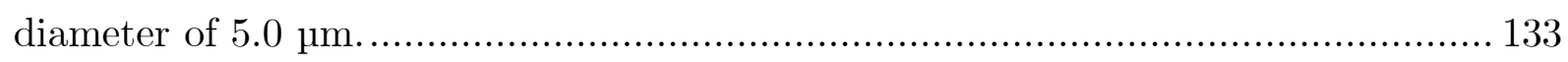

Figure 4.39: Exemplary force-distance curves, not leveled to the contact point, reveal a height difference between pore center and pore rim $h_{\mathrm{i}}$.

Figure 4.40: Exemplary RIfS diagram of spreading DOPC/DOTAP (8:2) SUVs and subsequent incubation with BSA.

Figure 4.41: Representation of a typical force-distance curve and the resulting slopes $\left(k_{\text {app }}\right)$ of a PSLB (incubated with BSA) indentation experiment with a trigger point of $1 \mathrm{nN}$. 136

Figure 4.42: Boxplots displaying the apparent spring constants $\left(k_{\text {app }}\right)$ of PSLBs with different diameter as a function of an attached MAC. 137

Figure 4.43: Correlation of determined apparent spring constants $\left(k_{\text {app }}\right)$ to different experimental days. 138

Figure 4.44: Influence of topographical AFM measurement on the morphology of attached F-actin networks revealed by fluorescence micrographs.

Figure 4.45: Boxplots displaying the lateral membrane tension $(\boldsymbol{\sigma})$ in both cases as a function of an attached MAC. 143

Figure 4.46: Fluorescence Airyscan micrographs of 3D F-actin network gels in dependence of fascin concentration. ${ }^{64]}$

Figure 4.47: Confocal Airyscan micrographs of attached pre-polymerized fascin treated networks. ${ }^{[64]}$.

Figure 4.48: Influence of fascin addition to membrane attached F-actin networks. ${ }^{[64]}$

Figure 4.49: Node densities of various F-actin networks showing the influence of fascin. ${ }^{[64]}$ 152

Figure 4.50: Time series of fluorescence Airyscan micrographs showing the influence of fascin. ${ }^{[64]}$ 153

Figure 4.51: Fluorescence Airyscan micrographs of 3D F-actin networks gels polymerized in absence or presence of $\alpha$-actinin. [64] 154

Figure 4.52: Influence of $\alpha$-actinin to minimal actin cortices in dependence of concentration and time. ${ }^{[64]}$ All images are $2 \mathrm{D}$ projections with averaged intensity of $Z$-stacks.

Figure 4.53: Strong rinsing leads to bundle displacement. 156

Figure 4.54: Time series of fluorescence Airyscan micrographs showing the influence of $\alpha$-actinin. ${ }^{[64]}$ 


\subsubsection{License Numbers of Retrieved Figures}

Figure 1.1:

Figure 1.3:

Figure 1.4:

Figure 1.6:

Figure 4.6 and 4.7:
4391210883920

4394601485481

4394601048422

4391210728632

4395920367193

\subsection{List of Schemes}

Scheme 3.1: Structural framework of TxR-DHPE............................................... 23

Scheme 3.2: Structural framework of Atto 488-maleimide. .................................... 24

Scheme 3.3: Structural framework of Alexa Fluor ${ }^{\circledR}$ 488-phalloidin.......................... 24

Scheme 3.4: Structural framework of Atto 390-DOPE........................................25

Scheme 3.5: Structural framework of Ni-NTA coordinatively binding nickel............29

Scheme 3.6: Reaction mechanism of the thiol-maleimide-addition........................... 32

\subsection{List of Tables}

Table 3.1: Structural framework and molecular masses of matrix lipids.................. 21

Table 3.2: Structural framework and molecular masses of receptor lipids.................22

Table 3.3: Structural framework and molecular masses of positively charged lipids. .23

Table 3.4: Buffer solutions used in case of each receptor lipid............................. 34

Table 3.5: Characteristics and origin of used tracer particles................................ 39

Table 4.1: Refractive indices and the resulting physical thicknesses $d$ of spread lipid bilayers.

Table 4.2: Determined segment lengths of the minimal data set via SOAX's and artificial retina's skeleton.

Table 4.3: Determined filament lengths of the minimal data set of SOAX [SOAX], the artificial retina [artificial retina] and the redrawn, thresholded SOAX-skeleton [SOAX skeleton]. $N$ displays the number of found filaments.... .96

Table 4.4: Exemplary comparison of SOAX's and artificial retina's skeletons with two benchmark networks traced by naked eye.

Table 4.5: Obtained mesh sizes $\xi$ compared with mesh sizes weighted by the area of the detected circles. 105

Table 4.6: Detected filament lengths $(\boldsymbol{L})$ utilizing a log-normal fit of the left-skewed length distribution.

Table 4.7: Height differences $h_{\mathrm{i}}$ between pore rim and pore center in dependency of pore size and attached MAC. 
Table 4.8: Alteration of filament lengths after addition of $36 \mathrm{nM}$ or $73 \mathrm{nM}$ fascin.. 151

\subsection{Abbreviations and Symbols}

$2 \mathrm{D}$

$\mathrm{ABP}$

$\mathrm{AC}$

ADP

$\mathrm{AF}$

$\mathrm{AFM}$

APS

ATP

$\mathrm{AU}$

BSA

c

cap-bio-DOPE

CCD

C-ERMAD

CF

CLAHE

CLSM

cp

d

$D$

DC-Chol

DOEPC

DOGS-Ni-NTA

DOL

DOPC

DOPE

DOTAP

DPPC

DTT

E. coli two-dimensional

actin binding protein

alternating current

adenosine diphosphate

actin filaments

atomic force microscopy

ammonium peroxydisulfate

adenosine triphosphate

airy unit

bovine serum albumin

concentration

1,2-dioleoyl-sn-glycero-3-phosphoethanolamine-N-(cap biotinyl)

charge-coupled device

$C$-terminal ezrin-radixin-moesin associated domain

correction factor

contrast-limited adaptive histogram equalization

confocal laser scanning microscopy

contact point

thickness

distance, deflection or diffusion coefficient

$3 \beta$-[N-( $\mathrm{N}^{\prime}, \mathrm{N}^{\prime}$-dimethylaminoethane)-carbamoyl]cholesterol

1,2-dioleoyl-sn-glycero-3-ethylphosphocholine

1,2-dioleoyl-sn-glycero-3-[(N-(5-amino-1-carboxypentyl)

iminodiacetic acid)succinyl] (nickel salt)

degree of labeling

1,2-dioleoyl-sn-glycero-3-phosphocholine

1,2-dioleoyl-sn-glycero-3-phosphoethanolamine

1,2-dioleoyl-3-trimethylammonium-propane

1,2-dipalmitoyl-sn-glycero-3-phosphocholine

dithiothreitol

Escherichia coli 


\begin{tabular}{|c|c|}
\hline EDM & Euclidian distance map \\
\hline ERM & ezrin-radixin-moesin \\
\hline$F$ & force \\
\hline F-actin & filamentous actin \\
\hline FDC & force-distance-curve \\
\hline FERM & four-point-one and ERM \\
\hline FMNL & formin-like protein \\
\hline fps & frames per second \\
\hline FRAP & fluorescence recovery after photobleaching \\
\hline$f_{\text {rep }}$ & reptation frequency \\
\hline FV & FluoView \\
\hline FWHM & full width at half maximum \\
\hline$G^{*}, G^{\prime}, G^{\prime \prime}$ & shear, storage and loss moduli \\
\hline$G_{0}$ & plateau modulus \\
\hline GaAsP & gallium-arsenide phosphide \\
\hline G-actin & globular actin \\
\hline GUV & giant unilamellar vesicle \\
\hline $\mathrm{h}$ & hour \\
\hline $\operatorname{His}_{n}-\operatorname{tag}$ & polyhistidine-tag with $\mathrm{n}$ histidine residues \\
\hline$I$ & intensity, light \\
\hline IF & intermediate filaments \\
\hline IPTG & isopropyl $\beta$-D-1-thiogalactopyranoside \\
\hline ITO & indium-tin-oxide \\
\hline$k_{\mathrm{app}}$ & apparent spring constant \\
\hline$k_{\mathrm{B}} T$ & Boltzmann constant \\
\hline$K_{\mathrm{d}}$ & dissociation constant \\
\hline$k_{\mathrm{off}}$ & unbinding rate constant \\
\hline$L$ & filament length \\
\hline LB & lysogeny broth \\
\hline LoG & Laplace of Gaussian \\
\hline$l_{\mathrm{p}}$ & persistence length \\
\hline$M$ & molar mass \\
\hline$m$ & mass \\
\hline MAC & minimal actin cortex \\
\hline$M_{\mathrm{av}}$ & average molecular mass \\
\hline $\min$ & minute \\
\hline
\end{tabular}




\begin{tabular}{|c|c|}
\hline MSD & mean squared displacement \\
\hline MT & microtubule \\
\hline$n$ & refractive index \\
\hline NA & numerical aperture \\
\hline$N$-ERMAD & $N$-terminal ezrin-radixin-moesin associated domain \\
\hline Ni-NTA & nickel nitrilotriacetic acid \\
\hline N-WASP & neuronal Wiskott-Aldrich syndrome protein \\
\hline $\mathrm{OD}_{600}$ & optical density at wavelength $600 \mathrm{~nm}$ \\
\hline$O T$ & optical thickness \\
\hline PBS & phosphate-buffered saline \\
\hline $\mathrm{P}_{\mathrm{i}}$ & inorganic phosphate \\
\hline PMT & photomultiplier tubes \\
\hline POPC & 1-palmitoyl-2-oleoyl-glycero-3-phosphocholine \\
\hline PSF & point-spread-function \\
\hline PSLB & pore spanning lipid bilayer \\
\hline PSM & pore spanning membrane \\
\hline $\operatorname{PtdIns}[4,5] \mathrm{P}_{2}$ & L- $\alpha$-phosphatidylinositol-4,5-bisphosphate \\
\hline QI & quantitative imaging \\
\hline$r$ & radius \\
\hline RIfS & reflectometric interference spectroscopy \\
\hline ROI & region of interest \\
\hline rpm & revolutions per minute \\
\hline $\mathrm{RT}$ & room temperature \\
\hline s & Second or path length \\
\hline SAM & self-assembled monolayer \\
\hline SAXS & small-angle x-ray scattering \\
\hline sCMOS & scientific complementary metal-oxide-semiconductor \\
\hline SDS & sodium dodecyl sulfate \\
\hline SDS-PAGE & sodium dodecyl sulfate--polyacrylamide gel electrophoresis \\
\hline SEM & scanning electron microscopy \\
\hline SLB & supported lipid bilayer \\
\hline $\mathrm{SOAC}$ & stretching open active contour \\
\hline $\mathrm{SOC}$ & super optimal broth with catabolite repression \\
\hline SUV & small unilamellar vesicle \\
\hline$t$ & time \\
\hline TEMED & $N, N, N^{\prime}, N^{\prime}$-tetramethylethane-1,2-diamine \\
\hline
\end{tabular}




$\begin{array}{ll}\text { tp } & \text { trigger point } \\ \text { TxR } & \text { Texas Red } \\ \text { TxR-DHPE } & \text { Texas Red-1,2-dhexadecanoyl-sn-glycero-3- } \\ & \text { phosphoethanolamine } \\ \text { UV } & \text { ultraviolet } \\ V, V & \text { volume } \\ \text { VASP } & \text { vasodilator-stimulated phosphoprotein } \\ \text { Vis } & \text { visible } \\ \text { VPT } & \text { video particle tracking } \\ \text { VVCA } & \text { verprolin, verprolin, central, acid - actin binding domain of } \\ & \text { N-WASP protein } \\ W & \text { weight } \\ \text { WASP/WAVE } & \text { Wiskott-Aldrich Syndrome protein } \\ \mu & \text { mean } \\ \mu^{*} & \text { median } \\ \chi & \text { percentage } \\ \lambda & \text { wavelength } \\ \lambda_{\text {em }} & \text { emission wavelength } \\ \lambda_{\text {ex }} & \text { excitation wavelength } \\ v & \text { node density } \\ \sigma & \text { filament thickness, standard deviation or membrane tension } \\ \sigma^{2} & \text { multiplicative standard deviation } \\ & \\ & \end{array}$

\subsection{Chemicals and Consumables}

$2 \mu \mathrm{m}$ silica particles

(non-fluorescent)

$2 \mu \mathrm{m}$ polystyrene beads

(fluorescent)

$\alpha$-actinin protein

$\operatorname{Acylamid}(\mathrm{AA})-\operatorname{mix}$

agar

Alexa Fluor ${ }^{\circledR}$ 488-phalloidin

APS
Bangs Labs, IN, USA

Sigma-Aldrich, Taufkirchen, Germany

Lyophilized powder, Cytoskeleton, Denver, CO, USA

Sigma-Aldrich, Taufkirchen, Germany

Merck KGaA, Darmstadt, Germany

Invitrogen, Carlsbad, CA, USA

Sigma-Aldrich, Taufkirchen, Germany 
argon

ATP

Atto 390-DOPE

Atto 488-maleimide

bromophenol blue

BSA

$\mathrm{CaCl}_{2}$

cantilever MLCT

cap-bio-DOPE

Chloramphenicol

chloroform

Coomassie Brilliant Blue G-250

DC-Chol

DOEPC

DOPC

DOTAP

DTT

E. coli BL21(DE3)pLysS

EDTA

fascin protein

ethanol p.a.

G-actin protein

glucose

glycerol

glycine

gold $(99.99 \%)$

guanidine carbonate

$\mathrm{H}_{2} \mathrm{O}_{2}(25 \%)$

$\mathrm{HCl}$

HEPES

imidazole

IPTG
Air Liquide Deutschland GmbH, Düsseldorf, Germany

Sigma-Aldrich, Taufkirchen, Germany

Atto-tec, Siegen, Germany

Atto-tec, Siegen, Germany

VWR International, Darmstadt, Germany

Carl Roth GmbH, Karlsruhe, Germany

Merck KGaA, Darmstadt, Germany

Bruker AFM probes, Calle Tecate, CA, USA

Avanti Polar Lipids, Alabaster, AL, USA

Sigma-Aldrich, Taufkirchen, Germany

VWR International, Darmstadt, Germany

Carl Roth GmbH, Karlsruhe, Germany

Avanti Polar Lipids, Alabaster, AL, USA

Avanti Polar Lipids, Alabaster, AL, USA

Avanti Polar Lipids, Alabaster, AL, USA

Avanti Polar Lipids, Alabaster, AL, USA

Sigma-Aldrich, Taufkirchen, Germany

Invitrogen, Carlsbad, CA, USA

Carl Roth GmbH, Karlsruhe, Germany

Lyophilized powder, Hypermol EK, Bielefeld, Germany

VWR International, Darmstadt, Germany

Lyophilized powder, Cytoskeleton, Denver, CO, USA

Carl Roth GmbH, Karlsruhe, Germany

Merck KGaA, Darmstadt, Germany

Merck KGaA, Darmstadt, Germany

Allgemeine Gold- und

Silberscheideanstalt, Pforzheim, Germany

Sigma-Aldrich, Taufkirchen, Germany

Merck KGaA, Darmstadt, Germany

Merck KGaA, Darmstadt, Germany

Carl Roth GmbH, Karlsruhe, Germany

Sigma-Aldrich, Taufkirchen, Germany

Sigma-Aldrich, Taufkirchen, Germany 
isopropyl alcohol

ITO slides

Kanamycin

$\mathrm{KCl}$

$\mathrm{KH}_{2} \mathrm{PO}_{4}$

methanol

$\mathrm{MgCl}_{2}$

microscopy slides

Mucasol ${ }^{\circledR}$

Na-citrate

$\mathrm{Na}_{2} \mathrm{HPO}_{4}$

$\mathrm{NaCl}$

$\mathrm{NaN}_{3}$

neutravidin

$\mathrm{NH}_{3}(30 \%)$

Ni-NTA agarose

Parafilm ${ }^{\circledR}$

poly-D-lysin

POPC

porous $\mathrm{SI}_{3} \mathrm{~N}_{4}$ substrates

PtdIns $[4,5] \mathrm{P}_{2}$

SDS

silicon wafers

sucrose

TEMED

Tris/ $\mathrm{HCl}$

tryptone

TxR-DHPE

ultrapure $\mathrm{H}_{2} \mathrm{O}$

yeast extract

$\beta$-mercaptoehtanol
Merck KGaA, Darmstadt, Germany

Präzisions Glas \& Optik GmbH, Iserlohn,

Germany

Sigma-Aldrich, Taufkirchen, Germany

Merck KGaA, Darmstadt, Germany

Merck KGaA, Darmstadt, Germany

Carl Roth GmbH, Karlsruhe, Germany

Merck KGaA, Darmstadt, Germany

Thermo Fisher Scientific, Waltham, MA, USA

Merck KGaA, Darmstadt, Germany

Merck KGaA, Darmstadt, Germany

Merck KGaA, Darmstadt, Germany

VWR International, Darmstadt, Germany

Merck KGaA, Darmstadt, Germany

Thermo Fisher Scientific, Waltham, MA, USA

VWR International, Darmstadt, Germany

Qiagen GmbH, Venlo ,Netherlands

Pechiney Plastic Packaging, Chicago, IL, USA

Sigma-Aldrich, Taufkirchen, Germany

Avanti Polar Lipids, Alabaster, AL, USA

fluXXion, Eindhoven, Netherlands

Aquamarijn, Zutphen, Netherlands

Avanti Polar Lipids, Alabaster, AL, USA

Merck KGaA, Darmstadt, Germany

Silicon Materials, Inc., Glenshaw, PA, USA

Carl Roth GmbH, Karlsruhe, Germany

Sigma-Aldrich, Taufkirchen, Germany

Carl Roth GmbH, Karlsruhe, Germany

Carl Roth GmbH, Karlsruhe, Germany

Sigma-Aldrich, Taufkirchen, Germany

Millipore, Billerica, MA, USA

Carl Roth GmbH, Karlsruhe, Germany

Carl Roth GmbH, Karlsruhe, Germany 


\subsection{List of Devices and Software}

Atomic Force Microscopes

MFP-3D classic

Nanowizard 4
Asylum Research, Santa Barbara, CA, USA

JPK Instruments, Berlin, Germany

\section{Confocal Laser Scanning Microscopy (CLSM)}

FluoView 1200

Olympus Europe SE \& Co.KG, Hamburg, Germany

LSM 880 Examiner

Carl Zeiss Microscopy GmbH, Oberkochen, Germany

\section{Reflectometric Interference Spectroscopy (RIfS)}

NanoCalc-2000, SD2000

Ocean Optics, Dunedin, FL, USA

spectrometer

Ismatec $795 \mathrm{C}$ peristaltic pump

IDEX Health \& Science, Wertheim, Germany

\section{Surface Coating}

Sputtercoater

Vaporization System MED020

\section{Other Devices}

frequency generator Agilent $3220 \mathrm{~A}$

NanoDrop 2000c

pH-meter Calimatic 766

plasma cleaner PDC 32 G-2

tip sonifier Sonoplus HD2070

Ultrapure water system

ultrasonic bath Sonorex RK $255 \mathrm{H}$

vacuum drying oven VD23
Cressington, Dortmund, Germany

Leica Microsystems GmbH, Wetzlar, Germany
Agilent Technologies, Santa Clara, CA, USA

Thermo Fisher Scientific, Waltham, MA, USA

Knick, Berlin, Germany

Harrick Plasma, Ithaca, NY, USA

Bandelin, Berlin, Germany

Millipore, Billerica, MA, USA

Bandelin, Berlin, Germany

Binder, Tuttlingen, Germany

\section{Software}

MATLAB 2016b

MathWorks, Natick, MA, USA

Spectra Suite

Ocean Optics Inc., Dunedin, FL, USA

Andor Solis

Gwyddion 2.45

Oxford Instruments plc, Abingdon, UK

http://gwyddion.net 
ImageJ (Fiji) 1.51f

SOAX 3.6.1

PYTHON 2.7.14

ZEN 2.3 light (blue), ZEN black $2.3 \mathrm{SP} 1$

Origin Pro $8.5 \mathrm{G}$ http://www.fiji.sc

http://www.cse.lehigh.edu/

idealab/soax/downloads.html

http://www.python.org

Carl Zeiss Microscopy GmbH, Oberkochen, Germany

OriginLab Corporation, Northhampton, MA, USA 


\section{Curriculum Vitae}

\section{Personal Data}

Name: $\quad$ Markus Schön

Date of Birth: $\quad 17^{\text {th }}$ August 1989

Place of Birth: Kassel, Germany

Citizenship: German

\section{Education}

2014-2018 PhD thesis at the Georg-August-University Göttingen (Prof. Dr. C. Steinem. Title of thesis: Self-Organization and Mechanics of Minimal Actin Cortices attached to artificial bilayers.

2012-2014 Graduate studies in chemistry at the Georg-August-University Göttingen (Master of Science). Title of master thesis: Quantifizierung der Aktivität von Dermcidin in lösungsmittelfreien Lipidmembranen.

2008-2012 Undergratuate studies in chemistry at the Georg-August-University Göttingen (Bachelor of Science). Title of bachelor thesis: Untersuchung der Interaktion von c-Polycystin-2 mit PIGEA-14 mittels Quarzmikrowaagetechnik.

2005-2008 Hessisches Landesabitur at the Albert-Schweitzer-Schule Kassel.

\section{Scientific Contributions}

\section{Publications}

2018 M. Schön, I. Mey and C. Steinem, Influence of cross-linkers on ezrin-bound minimal actin cortices, submitted to Prog. Biophys. Mol. Biol., 2018.

2018 H. Nöding, M. Schön, C. Reinermann, N. Dörrer, A. Kürschner, B. Geil, I. Mey C. Heussinger, A. Janshoff and C. Steinem, Rheology of Membrane-Attached Minimal Actin Cortices, J. Phys. Chem. B, 2018, 122, 4537-4545. 
2018 A. Bartsch, S. Llabrés, F. Pein, C. Kattner, M. Schön, M. Diehn, M. Tanabe, A. Munk, U. Zachriae and C. Steinem, High-resolution experimental and computational electrophysiology reveals weak betalactam binding events in the porin PorB, submitted to Sci. Rep., 2018.

\section{Oral Presentations}

2016

2016

CRC 937 Summer School, talk with H. Nöding, Heilbad Heiligenstadt. Self-organization and mechanics of actin networks attached to artificial membranes.

CRC 755/803/937 symposium 'Biological Dynamics from Microscopic to Mesoscopic Scales', talk with H. Nöding, Grimma. Self-organization and mechanics of actin networks attached to artificial membranes.

\section{Poster Presentations}

$11^{\text {th }}$ European Biophysics Congress, Edinburgh, Scotland. Mimicking the cellular cortex: Artificial F-actin networks physiologically attached to lipid bilayers.

CRC 937 symposium 'Physics meets Medicine - The Heart of Active Matter', Göttingen. Self-organization and mechanics of F-actin networks attached to artificial membranes.

$60^{\text {th }}$ Annual meeting of the Biophysical Society, Los Angeles, USA. Self-organization and mechanics of F-actin networks attached to artificial membranes.

2016 Meeting of the German Biophysical Society and satellite meeting, Erlangen. Investigating the Ezrin-mediated attachment of F-actin networks.

$201510^{\text {th }}$ European Biophysics Congress, Dresden. Self-organization and mechanics of actomyosin networks attached to artificial membranes. Bad Honnef. Self-organization and mechanics of actomyosin networks attached to artificial membranes.

\section{Awards}

Poster prize at the $11^{\text {th }}$ EBSA, Edinburg, Scotland.

2016 Poster prize at the meeting of the German Biophysical Society and satellite meeting, Erlangen. 


\section{Danksagung}

An erster Stelle möchte ich mich recht herzlich bei Prof. Dr. Claudia Steinem für die Betreuung dieser Doktorarbeit, den zahlreichen Diskussionen, das spannende Thema und die fortlaufende Unterstützung während der gesamten Promotionszeit bedanken.

Des Weiteren möchte ich mich bei Prof. Dr. Sarah Köster und Dr. Florian Rehfeldt für die gute Betreuung, die angeregten Diskussionen und gute Ratschläge im manchmal recht turbulenten Thesis Committee danken. Prof. Dr. Sarah Köster danke ich außerdem für die Übernahme der Zweitkorrektur dieser Arbeit. Prof. Dr. Silvio Rizzoli, Dr. Sebastian Kruss und Prof. Dr. Michael Meinecke danke ich für die Bereitschaft meine Prüfung als Mitglieder des Examination Boards mitzugestalten.

Allen Beteiligten des Projekt A17 aus dem SFB 937 möchte ich für den guten Austausch in zahllosen Meetings und die gute Zusammenarbeit danken. Speziell Prof. Dr. Andreas Janshoff für die vielen Diskussionen rund um Beads, Poren und manchmal auch das runde Leder. Ein großer Dank geht dabei an Burkhard, Filip und Nils. Durch die Entwicklung der Artificial Retina hat das Projekt und meine Arbeit eine neue, vielversprechende Richtung eingenommen. In dem Sinne möchte ich auch dem AK Janshoff danken für die Teilzeit-Aufnahme als gefühlt letzter „Steinhoff“. Dem gesamten SFB 937 gebührt ein großer Dank. Auf dem ein oder anderen Symposium sind tolle Ideen im Austausch entstanden, die mir und dem Projekt oft weitergeholfen haben.

Helen, dir möchte ich für die wunderbare und sehr gute Kooperation danken. Selbst in den Momenten, wo mal wieder gar nichts klappte, warst du die Ruhe in Person und ich bin mir sicher, dass es keine Selbstverständlichkeit ist so offen und vertraut miteinander arbeiten zu können. Ich bin unglaublich froh, dass du mit mir an diesem Projekt gearbeitet hast.

Ein weiteres, großes „Danke“ geht auch an unsere Sekretärinnen und Eva aus dem SFB Sekretariat. Ihr habt die komplizierte Uni-Bürokratie immer ein gutes Stück vereinfacht und ohne euch wäre bis heute nicht klar, wie viele Vergleichsangebote ich für Abrechnungen brauche. Auch dem GGNB-PBCS Office um Antje und Frauke möchte ich danken, dass ihr immer mit Rat und Tat zur Seite steht. Auch Holger Tucholla gebührt Dank, da er mir das Leben in mancher Hinsicht doch viel leichter gemacht hat.

Meinen Dank möchte ich auch dem AK Steinem aussprechen. Es ist ein wunderbares Gefühl Teil davon zu sein und zu sehen, wie hier jedes Rädchen ineinandergreift. Ihr seid da, wenn gerade mal alles den Bach herunterläuft und habt sämtliche Launen ertragen. Danke für all die guten, weiterbringenden Unterhaltungen, genauso wie für stumpfsinnige Diskussionen an Grill-, Film- oder sonstigen Abenden. 
Jutta danke ich außerdem für jegliche Unterstützung im Labor, selbst wenn ich mal wieder vergessen habe die notwendigen Details vorher aufs Papier zu bringen...

Ingo, dir danke ich auch wirklich von Herzen dafür, dass du immer das richtige Gespür hast, wann man Unterstützung und wann einen Tritt in den Allerwertesten braucht. Und wann man manchmal einfach nur Lust hat über das kleine Wörtchen unique zu streiten. Danke dafür und ich bin froh, dass du jetzt auf immer Bunt trägst!

Meinen Studenten Peter, Dominik, Aileen und Malte möchte ich danken, dass sie ein Teil dieser Arbeit waren. Es war wunderbar mit euch die Freuden, aber auch das Leid dieses Themas zu teilen und mitzuerleben, wie ihr euch mit diesem Thema auch weiterentwickelt habt. Ich bin mir sicher, ihr macht euren Weg auch!

Ein großer Dank geht auch an alle Korrekturleser, die sich mit meinem zum Teil doch sehr holprigen Englisch befasst haben und mich manchmal am Liebsten mit Kommata bewerfen wollten.

Ein riesengroßer Dank gilt auch zwei besonders wichtigen Menschen in meinem Leben. Jonas, dir danke ich für alles, was wir während dieser Promotion miteinander erlebt haben. Dafür, dass wir uns über jedes kleine Detail unserer Arbeiten, dem Arbeitskreis und auch aus dem Leben austauschen konnten. Auch dir Caro danke ich für die stetige Teilnahme an meinem Leben und damit auch an dieser Arbeit. Ich bin dir unendlich dankbar, dass du immer wieder schreibst, auch wenn ich (mal wieder) vergesse mich bei zu melden.

Generell gebührt meinen Freunden, Thilo, Basti, Sven, der gesamten Assiyolo-Gang, der Jungs vom Eishockey, meiner kleinen, aber feinen Semesterrunde um Martin, Martin und Ali und den SVG-„Brudis“ ein großer Dank, dass sie immer da waren. Auf euch alle kann ich mich verlassen und ich bin froh darum!

Meiner Familie, dem gesamten Schön/Wieder-Clan, möchte ich für den großen Rückhalt in den letzten Jahren danken. Zu guter Letzt gebührt der größte Dank den vier wichtigsten Menschen in meinem Leben: Zuerst meinen Eltern und meinem Bruder. Ohne Euch wäre das alles nicht möglich gewesen, ihr habt mir den Weg hierhin geebnet und wart stets mein größter Rückhalt. Und Vera, die mich in den letzten Jahren in gleichem Maße unterstützt hat und gerade in der schwierigen Zeit des Schreibens mir das Leben erleichtert hat und immer verständnisvoll mit meinen Launen umgegangen ist. Danke für Alles! 